\title{
IntechOpen
}

\section{Pediatric Surgery, Flowcharts and Clinical Algorithms}

\author{
Edited by Sameh Shehata
}

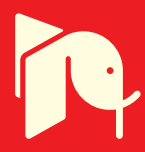





\section{Pediatric Surgery, Flowcharts and Clinical Algorithms \\ Edited by Sameh Shehata}



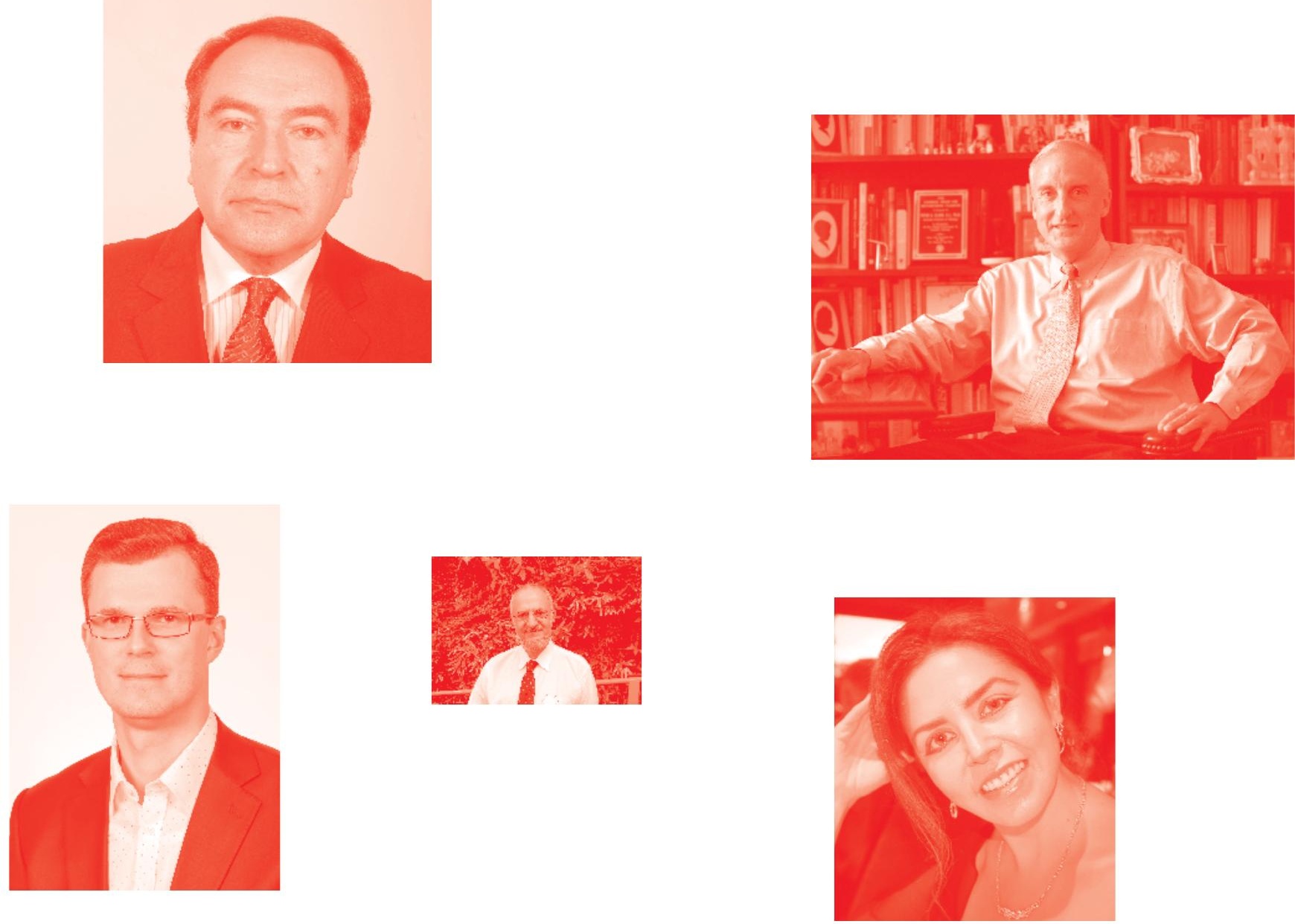

Supporting open minds since 2005
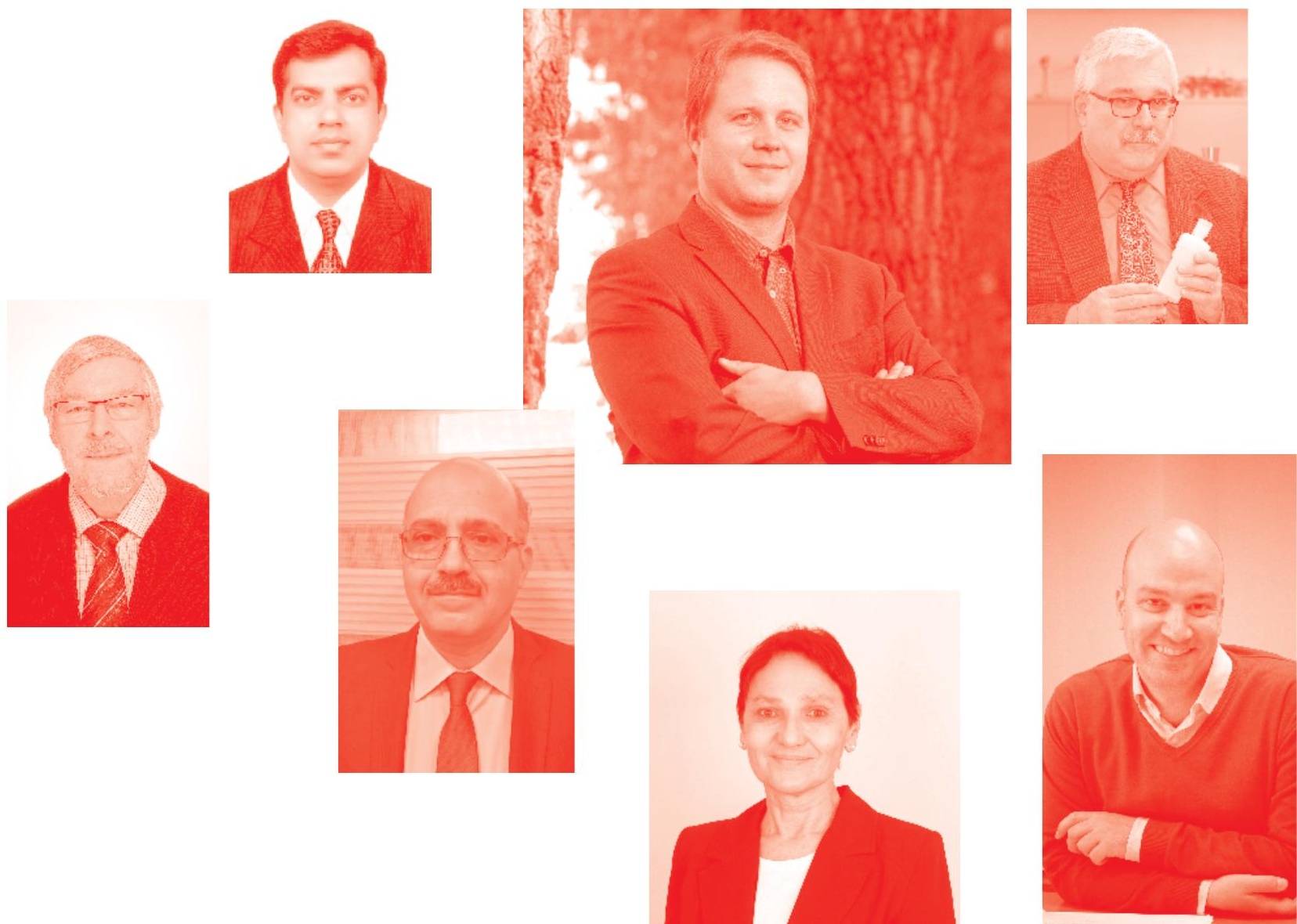
Pediatric Surgery, Flowcharts and Clinical Algorithms http: //dx. doi . org/10.5772/intechopen. 78837

Edited by Sameh Shehata

\section{Contributors}

Omogiade Udefiagbon, Owolabi Oni, Hasan Özkan Gezer, Alaa Obeida, Aly Shalaby, Samuel OseiNketiah, Willam Appeadu-Mensah, Ahmed Abdelghffar Helal, Ameen Alsaggaf, Yazeed Owiwi, Syed Salahuddin, Enaam Raboei, Mazen Zaidan, Alaa Ghallab, Rita Verma, Archana Kota

() The Editor(s) and the Author(s) 2019

The rights of the editor(s) and the author(s) have been asserted in accordance with the Copyright, Designs and Patents Act 1988. All rights to the book as a whole are reserved by INTECHOPEN LIMITED . The book as a whole (compilation) cannot be reproduced, distributed or used for commercial or non-commercial purposes without INTECHOPEN LIMITED's written permission. Enquiries concerning the use of the book should be directed to INTECHOPEN LIMITED rights and permissions department (permissions@intechopen.com).

Violations are liable to prosecution under the governing Copyright Law .

\section{(cc) BY}

Individual chapters of this publication are distributed under the terms of the Creative Commons Attribution 3.0 Unported License which permits commercial use, distribution and reproduction of the individual chapters, provided the original author(s) and source publication are appropriately acknowledged. If so indicated, certain images may not be included under the Creative Commons license. In such cases users will need to obtain permission from the license holder to reproduce the material. More details and guidelines concerning content reuse and adaptation can be found at http : //www . intechopen . com/copyright-policy . html.

\section{Notice}

Statements and opinions expressed in the chapters are these of the individual contributors and not necessarily those of the editors or publisher. No responsibility is accepted for the accuracy of information contained in the published chapters. The publisher assumes no responsibility for any damage or injury to persons or property arising out of the use of any materials, instructions, methods or ideas contained in the book.

First published in London, United Kingdom, 2019 by IntechOpen IntechOpen is the global imprint of INTECHOPEN LIMITED, registered in England and Wales, registration number: 11086078 , 7th floor, 10 Lower Thames Street, London, EC3R 6AF, United Kingdom

Printed in Croatia

British Library Cataloguing-in-Publication Data

A catalogue record for this book is available from the British Library

Additional hard and PDF copies can be obtained from orders@intechopen.com

Pediatric Surgery, Flowcharts and Clinical Algorithms

Edited by Sameh Shehata

p. $\mathrm{cm}$.

Print ISBN 978-1-78923-995-9

Online ISBN 978-1-78923-996-6

eBook (PDF) ISBN 978-1-83962-856-6 


\section{We are IntechOpen, \\ the world's leading publisher of Open Access books}

\section{Built by scientists, for scientists}

\section{$4,400+$}

Open access books available

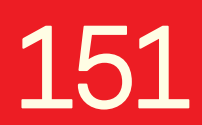

Countries delivered to

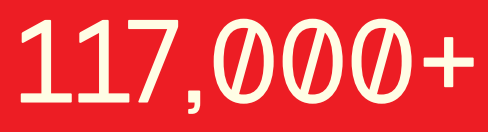

International authors and editors
$130 \mathrm{M}+$

Downloads

Our authors are among the

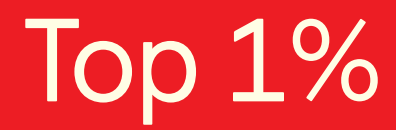

most cited scientists

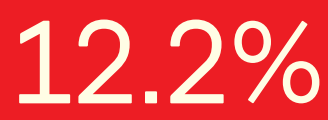

Contributors from top 500 universities

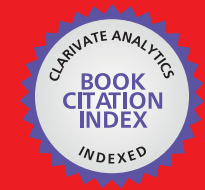

WEB OF SCIENCE ${ }^{\text {IM }}$

Selection of our books indexed in the Book Citation Index in Web of Science ${ }^{\mathrm{TM}}$ Core Collection (BKCI)

\section{Interested in publishing with us? \\ Contact book.department@intechopen.com}

Numbers displayed above are based on latest data collected.

For more information visit www.intechopen.com 



\section{Meet the editor}

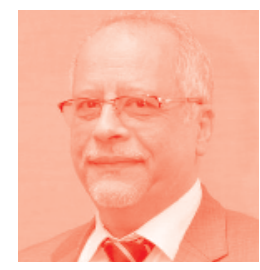

Prof Sameh Shehata is a professor and past chairman of the Pediatric Surgery Department, Faculty of Medicine, University of Alexandria, Egypt. He is the president-elect of the World Federation of Associations of Pediatric Surgery (WOFAPS) and the current president of the International Pediatric Endosurgery Group's (IPEG) Middle East chapter.

Dr. Shehata is the innovator of the laparoscopic traction technique for intra-abdominal testis, known as the "Shehata technique," which is widely practiced now in many centers worldwide.

Dr. Shehata is on the editorial boards of many national and international pediatric surgery journals, including JPS, Medicine, Annals of Pediatric Surgery, and the International Journal of Urology.

He has shared as a speaker, keynote speaker, moderator, and panelist at more than 250 national and international meetings. 



\section{Contents}

Preface

Section 1

Fetal Surgery

Chapter 1

Principles of Fetal Surgery

by Ahmed Abdelghaffar Helal

\section{Section 2}

Gastrointestinal Disorders

Chapter 2

Necrotizing Enterocolitis

by Rita Prasad Verma and Archana Kota

Chapter 3

Oesophageal Atresia: Drowning a Child in His/Her Own Saliva by Samuel Osei-Nketiah and William Appeadu-Mensah

Chapter 4

Meconium Ileus

by Udefiagbon Omogiade

Section 3

Hepatobiliary

Chapter 5

Pediatric Choledochal Cysts: Unknowns are Decreasing by Hasan Özkan Gezer

\section{Section 4}

Abdominal Wall Defects

Chapter 6

Management of Gastroschisis

by Alaa Obeida and Aly Shalaby 
Section 5

Minimally Invasive Surgery

Chapter 7

Single-Incision Pediatric Endosurgery (SIPES)

by Enaam Raboei, Ameen Alsaggaf, Yazeed Owiwi, Syed Salahuddin, Alaa Ghallab and Mazen Zidan 


\section{Preface}

Pediatric Surgery, Flowcharts and Clinical Algorithms is for readers interested in pediatric surgery. It provides updated information about some common topics together with suggested algorithms to assist the clinical management of different disease presentations.

The section on fetal surgery includes a chapter by Dr. Ahmed Abdelghaffar Helal that covers the most common anomalies encountered in fetal screening and possible fetal interventions as well as their success rates and the possible risks to the fetus and the mother.

The section on gastrointestinal disorders includes three chapters. "Gastrointestinal Surgical Disorders in Neonates" by Dr. Rita Verma covers the common gastrointestinal disorders in pediatric surgery, suggested diagnostic approaches, and updated management protocols together with prognosis.

“Oesophageal Atresia: Drowning a Child in His/Her Own Saliva” by Dr. Samuel Osei-Nketiah, and Dr. William Appeadu-Mensahm is a comprehensive review of esophageal atresia starting with a detailed description of the embryology, anatomy and physiology of the esophagus, and ending with antenatal and post-natal diagnosis and resuscitative management.

The chapter also describes different classifications systems and prognostic indicators as well as the surgical strategy for long-gap esophageal atresia, including early and late postoperative complications.

“Meconium Ileus" by Dr. Omogiade Ernest Udefiagbon covers the salient features of meconium ileus including an interesting algorithm to assist in decision making whether using operative or non-operative management.

The hepatobiliary section includes a chapter on "Choledochus Cysts" by Dr. Hasan Özkan Gezer that covers the history and etiology of these malformations and the different classification systems used over time. It discusses investigatory workup as well as the different management options for the different types of cysts, including open and laparoscopic approaches with discussion of the possible postoperative complications.

The section on abdominal wall defects includes a chapter on "Gastroschisis" by Dr. Alaa Obeida and Dr. Aly Shalaby. It covers the important aspects of gastroschisis including etiology and antenatal detection with special emphasis on gastroschisis in low- to middle-income countries. The chapter includes an important section on intraoperative decision making in some of the difficult comorbidities.

The section on minimally invasive surgery includes a chapter about "Single-Port Laparoscopic Surgery" by Dr. Enaam Raboei, which is about the tips and tricks of single-incision laparoscopic surgery, clearly showing the advantages and 
disadvantages of the technique, and some practical examples and personal experience for its application in common pediatric surgical procedures.

The chapter authors have made a great effort to bring the most updated information about these conditions to the pediatric surgeon to facilitate decision making and achieve best results and prognosis.

Sameh Shehata

Alexandria University,

Alexandria, Egypt 
Section 1

\section{Fetal Surgery}





\title{
Chapter 1
}

\section{Principles of Fetal Surgery}

\author{
Ahmed Abdelghaffar Helal
}

\begin{abstract}
Fetal therapy (in utero therapy) is a type of special therapy which aims to prevent or correct congenital anomalies in fetus, and prevents their severe consequences on later fetal development. It includes the use of in utero human fetal stem cell transplantation, fetal gene therapy and gene-editing technology as a new treatment for fetal genetic disorders. It started with open fetal surgery and then significantly advancing with innovations, toward minimally invasive fetal procedures, which are undoubtedly the future of fetal surgery, with the goal of providing the best possible fetal outcome, while minimizing the morbidity and mortality to the mother. The goal of fetal treatments is to decrease both fetal and maternal risks and prevent premature rupture of membranes. Fetal ultrasound and MRI are crucial for successful fetal interventions. Moreover, multidisciplinary fetal teams, including fetal surgeon, ultrasonographer, perinatologist, and anesthesiologist, are essential for optimum care to both mother and fetus. Finally, any new modality of fetal therapy must be thoroughly evaluated in animal models before clinical practice. In this chapter, we discuss the basic principles of fetal surgery, milestones of fetal surgery, specific fetal anomalies that are amenable for fetal surgery, successful fetal surgery criteria and future of fetal surgery.
\end{abstract}

Keywords: fetal therapy, in utero therapy, fetal team, minimally invasive fetal procedures

\section{Introduction}

Fetal surgery or in utero therapy tries to prevents or corrects multiple congenital anomalies in the fetus to prevent their severe consequences on later fetal development. It started with open fetal surgery then innovations toward minimally invasive procedures have occurred. In this chapter, we discuss the basic principles of fetal surgery, the general history of its development, important specific conditions and procedures used to treat them, and the future of the field.

\section{Key points}

- The goal of fetal treatments is to decrease both fetal and maternal risk and prevent premature rupture of membranes.

- Real-time ultrasound and fetal MRI is crucial to the implementation and success of fetal procedures. 
- Multidisciplinary fetal teams, including a fetal surgeon, ultrasonographer, perinatologist, and anesthesiologist, are critical to the delivery of optimum care.

\section{History and general principles of fetal surgery}

In 1963 first fetal intervention was performed (Table 1), the first fetal transfusion was reported by Liley. He used Tuohy needle (size 16-G) into the fetal peritoneal space. He injected a contrast material into the amniotic cavity to localize the fetal abdomen and the swallowed contrast opacify the fetal bowel. In the 1970s, endoscopy was used for direct visualization of the fetus, and the first fetal blood sampling or biopsy tissue was reported, however because of the limited technical skill, the therapeutic uses were not applicable $[1,2]$. After that, with more use of ultrasound as non-invasive diagnostic tool, the use of diagnostic fetoscopy was replaced with percutaneous needle-based techniques under ultrasound guidance. In the 1980s open fetal surgery was started (direct exposure of the fetus by maternal laparotomy and hysterostomy), then open fetal surgery was replaced with a less invasive fetoscopy, where video camera was inserted inside the uterus under ultrasound guides. At first, fetoscopy was performed in amniotic fluid medium, using a single port to enter the uterine cavity and with a side way working channel. However, amniotic fluid medium poses many limitations for many fetal surgeries especially that require dissection and suture. Low quality images in the fluid medium, and any bleeding will prevent an adequate imaging, and it can end the procedure. Moreover, the "fluctuation" of the fetus during the intervention prevent maintenance of the ideal accessible position. In $2010 \mathrm{Kohl}$ et al. use low insufflation pressure carbon dioxide of amniotic cavity which was left with some amount of amniotic fluid [3-6].

\begin{tabular}{lc}
\hline First fetal intervention & 1963 \\
\hline Direct visualization of the fetus & 1970 \\
\hline Start of open fetal surgery & 1982 \\
\hline Thoraco-amniotic fetal shunt placement & 1987 \\
\hline Treatment of twin-twin transfusion syndrome by laser ablation & 1990 \\
\hline Closure of fetal myelomeningocele using fetoscopy & 1997 \\
\hline Treatment of fetal congenital diaphragmatic hernia by tracheal clipping (Fetendo technique) & 1997 \\
\hline Excision of fetal amniotic band using fetoscopy & 1997 \\
\hline $\begin{array}{l}\text { Treatment of fetal congenital diaphragmatic hernia by fetoscopic balloon tracheal occlusion } \\
\text { technique }\end{array}$ & 2001 \\
\hline
\end{tabular}

Table 1.

Time scales for important fetal surgeries.

\section{Milestones in development of fetal surgery}

Criteria for fetal surgery are summarized in Table 2.

\subsection{Ethical considerations}

The ethical issues in the field of fetal surgery are complex because the medical intervention is always invasive, often experimental, involves at least two patients the mother and fetus, and the success rate is difficult to measure. On the 


\begin{aligned} & \hline I. Prompt diagnosis of the pathology and associated anomalies. \\ & \hline II. Pathophysiology of the disease is documented, and overall prognosis is promising \\ & \hline III. No curative postnatal treatment. \\ & \hline IV. $\begin{array}{l}\text { Animal models prove feasibility of the in utero technique, preventing serious effects of the } \\ \text { pathology. }\end{array} \\ &$\hline V. $\begin{array}{l}\text { Fetal therapy performed in specialized multi-disciplinary fetal care centers within clear procedure } \\ \text { with local ethics committee approval and signed informed maternal or parent consent. }\end{array} \\ &$\hline\end{aligned}

Table 2.

Successful fetal surgery criteria.

other hand, strong evidence on the benefits of fetal surgery are not present, with many centers considered fetal surgery as an experimental technique to correct fetal anomalies. Controlled randomized studies to evaluate the effects of fetal surgeries on both mother and fetus are still needed. More often, doctors attempt fetal surgery in clinical settings without reporting post-operative outcomes in medical journals. The overall goal of fetal interventions is clear: to improve the health of fetus by intervening before birth to correct or treat prenatally diagnosed abnormalities. Mother and fetus that undergo these interventions must have the same protection afforded to other study participants, with detailed explanation of both short and long-term risks and benefits of these interventions on both the mother and the fetus. Therefore, diagnostic or therapeutic fetal intervention, cannot be performed without mother explicit informed consent (Table 2) [7-10].

\subsection{Surgical techniques and procedures}

\subsubsection{Surgical team}

In fetal surgery, there are complex diseases and two patients, so careful planning and open communication before, during, and after surgery between the members of the multidisciplinary care team are essential. The team must include pediatric surgery, obstetrics, pediatric anesthesia, obstetric anesthesia, cardiology, radiology, otolaryngology, neonatology, neonatal nursing, and operative room nursing $[11,12]$.

During any fetal procedures, the use of ultrasound will guide the pediatric surgeon and/or obstetrician and allow for monitor the fetus during surgery. The surgeon should actively communicate with the anesthesia team, as well as nursing and scrub staff, throughout the procedure. Also, the presence of knowledgeable technical support staff familial with the specialized equipment and instrument is essential $[13,14]$.

\subsubsection{Surgical approach}

Currently, fetal surgery can be classified into three broader areas; open fetal surgery, minimally invasive fetal surgery and EXIT procedures. Each procedure is subdivided into several subdivisions, in an attempt to treat a wide number of severe pathologies that would compromise the fetus. During minimally invasive fetal surgeries a small skin incision on the mother's abdomen was done. The location of the placenta, as well as the intrauterine pathology will guide the site of the planned incision. 1-2 mm instruments were used to access the fetus are to minimize maternal morbidity. Also, curved instruments may be used to avoid injury to anteriorly placed placenta. During fetal access, any present fluid (ascites, pleural effusions, 
cystic structures, or the bladder) are aspirated or shunted into the amniotic space. During fetal cardiac valvuloplasty and radiofrequency ablation for treatment of complicated twin gestation, needle-based access is very helpful [15-20].

\subsection{Fetoscopic procedures}

Usually all patients submitted for a fetoscopic procedure are often pre-medicated with a tocolytic agent. Local or regional anesthesia are usually used. The surgery can be performed in the surgical theater, labor or delivery unit, or in the ultrasound department (depends on gestational age of the fetus). The used instruments, particularly, endoscopes have undergone numerous evolution, based on prototypes developed in animal models. Fetoscopes diameters are between 1.0 and $2.0 \mathrm{~mm}$. Sharp trocars have been developed to accommodate the wide range of diameters used for different operations. Operative fetoscopy is a sonoendoscopic enterprise that has evolved so that the surgical team can see the ultrasound and fetoscopic images simultaneously. Basically, the ultrasound is used to identify an appropriate entry point to direct the trocar into the amniotic cavity, avoiding the placenta and the fetus as well as maternal organs, such as the bowel and bladder. However, some operators have documented the safety, in their hands, of a transplacental approach. Despite this experience, most operators still attempt to avoid the placenta. Nowadays, fetoscopic technique is indicated when direct visualization of the fetus (more than ultrasonography) is needed, as in treatment of cases of twin to twin transfusion syndrome, posterior urethral valves, constricting amniotic bands, and tracheal balloon occlusion for treatment of congenital diaphragmatic hernia. Fetoscopic procedures are performed using 1.2- to $3.0-\mathrm{mm}$ endoscopes. Pictured is a $3 \mathrm{~mm} 0^{\circ}$ endoscope, adjustable length, with a 1-mm working channel (Figure 1) [21-29].

\subsection{EXIT procedures}

EXIT procedures principles is to perform controlled delivery to allow for fetal intervention and establishment of airway prior to cord clamping/cutting. Indications of EXIT procedures includes severe airway obstruction or likelihood of cardiopulmonary insufficiency at birth. Cervical masses, congenital lung malformations (CLM), congenital high airway obstruction (CHAOS), pulmonary agenesis, transition to ECMO. It performed under general anesthesia (fetal anesthesia, uterine relaxation), with maintenance of placental circulation and dorsal supine leftward tilt. Steps includes; Pfannenstiel incision, then customized hysterotomy based on placental location, partial delivery of fetus, and placement of

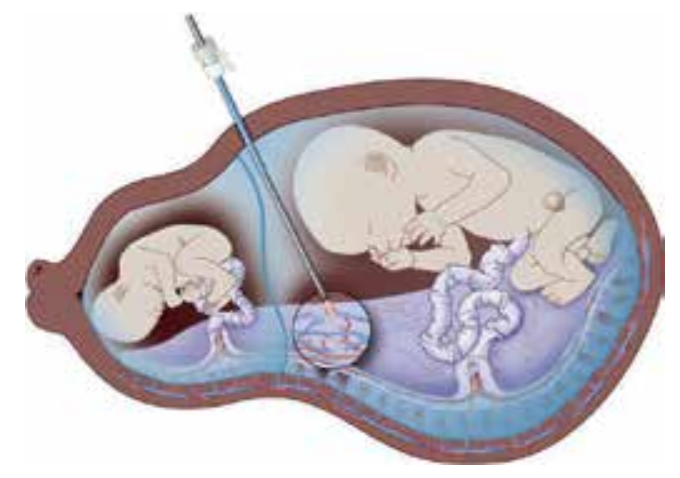

Figure 1.

Fetoscopic laser ablation of abnormal chorionic vessels for TTTS. 


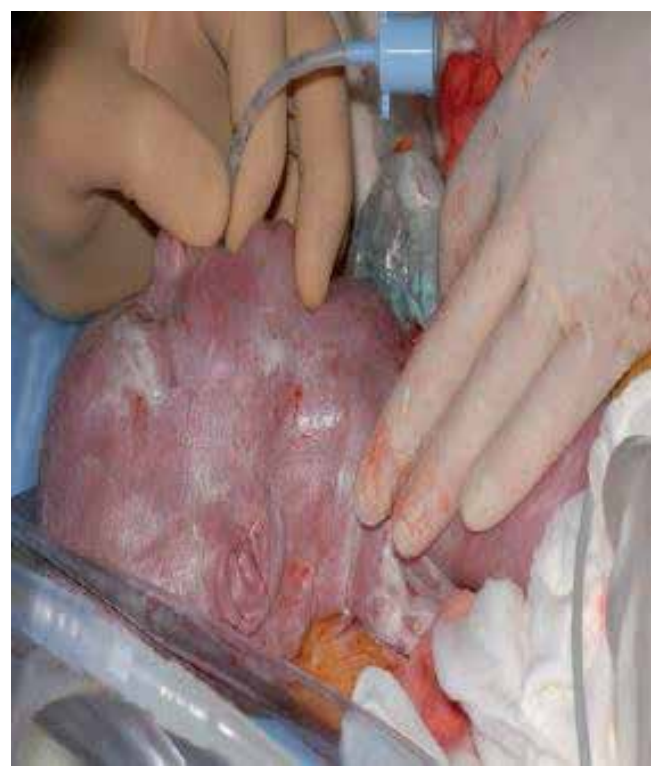

Figure 2.

Successful oro-tracheal intubation during the EXIT procedure.

monitors, fetal airway establishment during surgical intervention then delivery was completed with, transition of the baby to postnatal care, and finally completion of cesarean section (Figure 2) [30, 31].

\subsubsection{Anesthetic care}

Before anesthesia, all physiological changes associated with pregnancy must be considered. The effects of pregnancy on mother pulmonary and cardiovascular function must be considered. Adequate precautions should be taken to prevent hypoxemia and aspiration. The magnesium sulfate used in tocolysis may decreases capillary oncotic pressure and increases capillary permeability with increased risk of pulmonary edema. Aorto-caval compression must be prevented by using left uterine displacement. The doses of anesthetic drugs must be adjusted. Maternal local anesthesia can be effectively used for most needle-based and single port fetoscopic procedures. When multiple ports or caesarian section could be necessary, regional anesthesia; epidural or combined spinal epidural can be added. On the other hand, fetal anesthesia is indicated only for endoscopic procedures performed directly on the fetus. All fetal anesthetic drugs are typically administered through intramuscular route and consists of opiates and non-depolarizing muscle relaxants. Atropine is usually given to avoid fetal bradycardia. For placental or cord procedures with no direct fetal contact, the risk-benefit of fetal anesthesia should be weighed [32-35].

\section{Drawbacks of fetal surgery}

The drawbacks of fetal surgery, includes bleeding, amniotic fluid leak, chorioamnionic separation, chorioamnionitis, premature rupture of membranes, preterm labor, preterm birth, and fetal loss. Premature rupture of membranes, preterm labor is the most common complication of minimally invasive fetal surgery, with high morbidity, including oligohydramnios, chorioamnionitis, and preterm delivery. However, accurate analysis of the frequency of these complications are difficult 
due to variations in both the assessment of the complication as well as reporting methods. Factors increasing the risk during minimally invasive fetal procedures include the number of ports and the diameter of the used instruments. A systematic review of 1376 minimally invasive fetal procedures for lower urinary tract obstruction, and twin reversed arterial perfusion reported that increased diameter of the instrument and increased number of ports are major predictors of iatrogenic premature rupture of membranes [36-38].

Following open fetal procedure, risk of hysterotomy scar weakness may interrupt both current and future labor. Some cases of uterine scar rupture after open fetal procedure was reported, starting from the second trimester, may be caused only by the uterine distension (with no uterine contractions) which form significant risk to both mother and fetus. Moreover, maternal counseling about the risk of delivery complications must be considered. On the other hand, minimally invasive fetal procedures do not preclude vaginal delivery. However, long-term follow-up of subsequent pregnancies after these procedures is not available, beside the complications of repeat caesarian section, significant advantage of minimally invasive fetal procedures should be considered [39, 40].

\section{Certain problems amenable for fetal surgery}

Some important indications for fetal interventions are summarized in Table 3.

\subsection{Twin gestations}

\subsubsection{Twin-twin transfusion syndrome (TTTS)}

Monochorionic (shared) placenta with A-V, V-V, A-A connections and unbalanced flow between two twins

- Donor - low flow, oligohydramnios, high output heart failure, brain ischemia, small

- Recipient - fluid overload, polyhydramnios, congestive heart failure, hydrops fetalis, large

Prognosis: 80-90\% mortality for both if untreated.

\section{Fetal Surgery}

- High-volume amnioreduction historically (survival of at least one twin 60\%)

- Fetoscopic laser ablation of vascular connections

Offered to Stage II or greater

Selective A-V or nonselective

$76 \%$ single survivor, $36 \%$ dual survivors.

The donor twin usually develops hypovolemia, leading to oliguria and oligohydramnios from reduced renal perfusion, and the recipient twin suffers the consequences of hypervolemia, including polyuria and polyhydramnios. Both twins are at risk for significant morbidity [41, 42].

Basic principles for TTTS interventions are to prevent preterm delivery caused by polyhydramnios, through removing the excess amniotic fluid surrounding 
Principles of Fetal Surgery

DOI: http://dx.doi.org/10.5772/intechopen. 85883

\begin{tabular}{|c|c|}
\hline Fetal surgery & Why to interfere? \\
\hline \multicolumn{2}{|l|}{ I-Surgery on the fetus } \\
\hline Congenital diaphragmatic hernia & Avoidance of pulmonary hypoplasia and pulmonary hypertension \\
\hline Sacrococcygeal teratoma & Stop of steal phenomenon, avoid cardiac failure and polyhydramnios \\
\hline Thoracic space-occupying lesions & Avoid pulmonary hypoplasia and cardiac failure \\
\hline Lower urinary tract obstruction & Avoid renal failure and pulmonary hypoplasia \\
\hline Cardiac malformations & Avoid hypoplasia or progressing damage to developing heart \\
\hline Myelomeningocele & $\begin{array}{l}\text { Repair of exposed spinal nerves, stop cerebrospinal fluid leakage, } \\
\text { prevent hydrocephaly and hindbrain herniation }\end{array}$ \\
\hline \multicolumn{2}{|c|}{ II- Placenta, cord or membranes surgery } \\
\hline $\begin{array}{l}\text { Complicated monochorionic } \\
\text { pregnancies: }\end{array}$ & Arrest of feto-fetal transfusion and its consequences \\
\hline $\begin{array}{l}\text { Twin-twin transfusion syndrome } \\
\text { (TTTS) }\end{array}$ & Avoid preterm delivery \\
\hline $\begin{array}{l}\text { Twin-reversed-arterial-perfusion } \\
\text { sequence } \\
\text { (TRAP) and other discordant } \\
\text { anomalies }\end{array}$ & Avoid damage to co-twin \\
\hline $\begin{array}{l}\text { Twin-anemia polycythaemia } \\
\text { sequence }\end{array}$ & $\begin{array}{l}\text { In some conditions (TTTS/TRAP) reversal of cardiac failure and } \\
\text { polyhydramnios }\end{array}$ \\
\hline \multicolumn{2}{|c|}{ III- Selective intra-uterine growth restriction } \\
\hline Amniotic band syndrome & Avoid deformities and functional loss \\
\hline Chorioangioma & Avoid of cardiac failure, hydrops fetoplacental and polyhydramnios \\
\hline
\end{tabular}

Table 3.

Some important indications for fetal surgery.

the recipient twin, with improved fetal circulation by decreasing pressure on the chorionic plate. In 1990, fetoscopic laser was used to coagulate the crossing superficial blood vessels separating the 2 fetal circulations and destroying the inter-twin vessels that cause discordant twin-twin transfusion. Nowadays, laser ablation is the preferred treatment for TTTS between 16 and 26 weeks of gestation. The procedure is performed through a single uterine access site using a fetoscope and thin laser (Table 4) [43-45].

\subsubsection{Twin reversed arterial perfusion}

One normal twin acts as a "pump" for a cardiac, a cephalic twin via A-A anastomoses

\begin{tabular}{ll}
\hline I & Presence of Poly and oligohydramnios \\
\hline II & Stage I plus non-visualized bladder in donor twin \\
\hline III & $\begin{array}{l}\text { Stage II plus seriously abnormal Doppler (umbilical artery absent or reversed end-diastolic velocity, } \\
\text { ductus venosus reversed flow, pulsatile umbilical venous flow) }\end{array}$ \\
\hline IV & Stage III plus evidence of hydrops in either twin \\
\hline V & Fetal death \\
\hline
\end{tabular}

Table 4.

Staging of TTTS. 
Normal twin much like donor twin in TTTS with R/O high output heart failure, hydrops fetalis, $50 \%$ mortality

Fetal Surgery

- Open hysterotomy/delivery

- Fetoscopic ligation

- Bipolar cautery/harmonic scalpel division

- Thermal/laser coagulation

- RFA of acardiac/acephalic cord insertion

\subsubsection{Selective fetal reduction}

In addition to TTTS, other serious problem that can affect monochorionic twin pregnancies, includes severe intrauterine growth restriction, structural anomalies, twin anemia polycythemia sequence, and TRAP sequence, or a cardiac twinning. In some complicated monochorionic pregnancies, elective fetal reduction is recommended especially for high risk of hemodynamic compromise or intrauterine fetal death, aimed to prevent neurologic injury or demise to the co-twin. Fetal intra-cardiac potassium chloride injection is contraindicated in these pregnancies, because of risk of transmission between twins and selective termination must be performed with interruption of blood flow to the fetus. This interruption usually performed through ligation of the umbilical cord, fetoscopic laser coagulation, ultrasound-guided and bipolar cord coagulation. Selective fetal reduction was seriously indicated in complicated twin pregnancies what is TRAP sequence. In TRAP sequence, one twin is incompatible with life due to absent or rudimentary heart, as well as absence of other vital structures, as head (anencephaly). This twin usually has no placental blood supply, and it receives its blood supply directly through vascular connections from the second normal twin (acts as pump twin). Therefore, the normal twin will rapidly develop high-output heart failure, with more than $50 \%$ mortality rate. Selective fetal reduction aims to stop blood flow to incompatible with life twin, and save the life of normal (pump) twin. In the largest review from 12 fetal centers from the North American Fetal Therapy Network registry data, identified 98 patients who underwent percutaneous radio frequency ablation of a cardiac twin. In this series, the overall survival of the normal (pump) twin to 30 days was $80 \%$ [46-50].

\subsection{Lower urinary tract obstruction (LUTO)}

LUTO can be caused by stenosis of the urethral meatus, valves, urethral atresia, ectopic insertion of a ureter or peri-vesical tumors. Bladder shunts are effective for urine diversion, restoring amniotic fluid and thereby preventing pulmonary hypoplasia. Whether shunting effectively salvages renal function is uncertain. For that, prior accurate assessment of renal function is required. The actual anatomical cause of LUTO proved to be an important predictor. Posterior urethral valves do much better in the long run, while babies with urethral atresias or the Prune Belly phenotype do less well. At the moment, the two commonly used techniques, are percutaneous vesico-amniotic shunting, under ultrasound guide, where double pigtail stent is inserted, usually combined with amnio-infusion. The second procedure is fetal cystoscopy, where fetoscope is inserted into the fetal bladder, to diagnose the 
source of obstruction and to ablate PUV. The commonly used methods to ablate the valve, includes guide wires, hydro-ablation and laser-ablation. The first open clinical fetal surgical intervention for a case of lower urinary tract obstruction (LUTO), not eligible for shunt placement. Instead, fetal ureterostomies were successfully created. There were no maternal complications, but unfortunately the fetus never produced any urine. On the other hand, main complications of shunting include; failure to insert the catheter, occlusion of the catheter, dislocation, and sometimes fistula formation. In order to conclude and evaluate the results of in-utero VAS, and its longterm outcomes, randomized, controlled trial, "Percutaneous vesico-amniotic shunting versus conservative management for fetal Lower Urinary Tract Obstruction" (PLUTO), was performed in the United Kingdom, Ireland, and the Netherlands from 2006 to 2012. The study performed on 31 cases (16 submitted to VAS, 15 undergo conservative treatment). Study reported that fetal cystoscopy, although it is more invasive than VAS, it has the advantage of confirming the diagnosis of PUV, and more accurate in selection of patients who will benefit from valve ablation. In other multi-centric retrospective study includes 50 cases submitted to fetal cystoscopies for treatment of LUTO, 30 fetuses were diagnosed with PUV and were treated with laser-ablation. Other 13 fetuses were diagnosed with urethral atresia, 5 fetus diagnosed with urethral stenosis, and 2 fetuses diagnosed with trisomy 18 (not treated). The results of the 54 fetuses with normal karyotype were, 32.4 weeks mean delivery gestational age, and $34.8 \%$ overall 2 years' survival. For PUV patients treated with laser-ablation, $53.6 \% 2$ years' survival. Although 20\% (6 of the 30 ) developed recurrence of LUTO symptoms, and further fetal procedure was performed in $10 \%$ (3 patients). Postnatal ablation of PUV was needed in 10 of the 17 survivors. Normal renal function at 2 years of age, was achieved in $75 \%$ of infants with PUV (12 of the 16 ), which considered more promising than the $29 \%$ reported in the PLUTO trial with VAS. Reports up to date indicate that, minimally invasive fetal procedure (in selected cases of LUTO), can improve the survival when compared to expectant treatment. However, studies of long term renal function are less encouraging [51-56].

\subsection{Intrauterine myelo-meningocele (IMM)}

IMM, or Spina bifida, is defined as failure of complete closure of the neural tube with exposure of the spinal canal structures. Lumbar or cervical vertebral levels are the most commonly affected sites, however IMM can occur anywhere along the spine. Neurologic deficits with motor and somato-sensory abnormalities are the most feared complications. In addition, bowel and bladder function may be affected due to injury of autonomic nervous system. Moreover, mostly all patients with IMM will develop Arnold-Chiari II malformation affecting hindbrain, with non-communicating hydrocephalus, which requires ventriculo-peritoneal shunting. Although mortality of IMM was low in the perinatal period, its long-term neurologic morbidity may be fatal, and up to $30 \%$ of patients may die before adulthood [57-60].

\subsubsection{IMM repair}

At the moment, the compared outcomes of pre-natal versus post-natal repair of IMM showed that; although prenatal surgery has an increased risk of preterm delivery, pre-natal repair had significantly better outcomes than the post-natal repair. Pre-natal repair for IMM decrease the risk of death and subsequent needs for shunting (nearly at age of 12 months). Also, pre-natal repair improves scores of mental and motor function (at 30 months). However, pre-natal repair was associated with an increased risk of preterm delivery and uterine dehiscence at labor. Therefore, the potential benefits of pre-natal repair must be balanced against the risks of 
prematurity and maternal morbidity [61-65]. In a retrospective review of 54 children evaluated for lower extremity neuro-motor function and short-term ambulatory function following fetal myelomeningocele closure, they concluded that fetal myelomeningocele repair results in better than predicted lower extremity neuro-motor function at birth and short-term ambulatory status. However, these children continue to demonstrate movement incoordination which is characteristic for children with Spina-bifida. In retrospective study evaluated the incidence and clinical implications of the development of cutaneously derived intradural inclusion cysts (ICs) following fetal myelomeningocele closure through parental questionnaire. They concluded that intradural ICs can develop following fetal myelomeningocele repair. ICs long-term complications in these children may include deterioration of bladder function, and loss of lower-extremity function after fetal myelomeningocele closure. Koh et al. 2006 compared urodynamic findings in patients who underwent pre-natal closure of IMM with those of patients who underwent post-natal closure. All prenatally treated patients had lower lumbo-sacral lesions on neurological examination. In comparison, 39\% of post-natally treated patients showed lack of sphincter activity at newborn examination, with similar findings noted at 1-year evaluation. Regarding bladder function, all pre-natally treated patients showed detrusor overactivity, compared to $38 \%$ of post-natally treated patients, up to 1 -year evaluation. They concluded that fetal closure of IMM is associated with a higher incidence of complete denervation of the external urethral sphincter and detrusor over-activity compared to post-natal repair. Open fetal IMM closure has been extensively studied and its benefits to the fetus have been proven. Minimally invasive fetoscopic repair is technically difficult, with high risk of membrane separation and premature rupture of membrane, and its benefits to the fetus have not been proved. Therefore, minimally invasive IMM repair still needs further more studies to confirm its validity for clinical applications [66-70].

\subsection{Congenital diaphragmatic hernia $(\mathrm{CDH})$}

One in 2500 live infant births may be affected with CDH. It consists of abnormal defect in the fetal diaphragm, resulting in herniation of all or part of abdominal viscera into the thoracic cavity (according to the defect size). Pulmonary hypoplasia and pulmonary hypertension, usually present as a result of abnormal development of the lungs and pulmonary vasculature, this may cause persistent fetal circulation and respiratory failure with increased mortality rate. Despite great improvement in its diagnosis and treatment, infant mortality from isolated CDH still about 20-30\%. Poor prognostic indicators by ultrasonography include low lung-to-head ratio, liver herniation into the thoracic cavity, and low total lung volume detected by fetal MRI. Initial experimental studies using sheep models demonstrated that prenatal repair of the diaphragmatic hernia could reverse the pulmonary hypoplasia caused by a surgically created $\mathrm{CDH}$. The first clinical experience in humans likewise involved open fetal surgery. High fetal mortality rate is associated with this approach, because of umbilical vein kinking during reduction of the herniated liver into the fetal abdomen. Therefore, all recent studies consider that minimally invasive methods, taking the upper hand for treatment of $\mathrm{CDH}$, as it stimulates lung growth in utero, while open fetal repair of the diaphragmatic defect was abandoned from the clinical use. Preliminary experimental studies in fetal lambs were promising, demonstrating that fetal tracheal occlusion improved fetal lung growth and reducing the severity of pulmonary hypoplasia. In cases of severe $\mathrm{CDH}$, postnatal mortality appears to be significantly lower with in utero surgical intervention. Studies of fetal endoscopic tracheal occlusion (FETO) performed in cases of severe $\mathrm{CDH}$ have demonstrated a significantly higher survival rate compared with control fetuses that did not undergo 
FETO. Severe PAH occurred in $47 \%$ of fetuses that underwent FETO, but in $89 \%$ of patients in the prenatal expectant management group (Figure 3) [58, 71, 72].

So, fetal surgeons prefer minimally invasive procedures to avoid large hysterotomy and adequately visualize and access the fetal trachea. In 1997 the first fetal endoscopic (Fetendo) tracheal clipping, was done in human fetus, where maternal laparotomy was done, then 4 trocars was inserted through uterus to access and clip the fetal trachea. For the fear of serious complications of tracheal damage and vocal cord paralysis during clipping, fetoscopic balloon tracheal occlusion technique was introduced, in which no fetal neck dissection and only single uterine port was needed. Fetal endoscopic tracheal occlusion (FETO) is usually performed between 26 and 30 weeks of gestation., A trocar is placed through the maternal abdomen into the amniotic cavity guided by ultrasound, and fetoscope is inserted through the fetal mouth, then advanced into the fetal trachea. Once the carina has been retched, the balloon is inflating with physiologic solution and left just above the carina. The correct position is then checked by ultrasound imaging. In some studies, the tracheal balloon was removed at the time of delivery through ex utero intrapartum therapy. However, balloon removal before birth not only allows for the possibility of vaginal birth, but also was shown to increase pneumocyte cells differentiation type II, with increasing surfactant production. Currently, tracheal occlusion can be reversed in utero, by performing second fetoscopic procedure (typically at 34 weeks of gestation) $[73,74]$. A multicenter European series including 210 cases of FETO with severe CDH (liver up and lung-to-head ratio $\leq 1$ ) they reported $48.0 \%$ rate of survival to discharge, with $47.1 \%$ incidence of premature rupture of membrane. Up to date meta-analysis comparing survival outcome between FETO and a contemporary control group, reported that FETO improves survival compared with standard perinatal care in fetuses with isolated $\mathrm{CDH}$ and severe pulmonary hypoplasia (lungto-head ratio $\leq 1$ ). $46.3 \%$ of fetuses (Fifty-one of 110 ) who had undergone FETO survived to discharge, compared with $5.9 \%$ (6 of 101) in the control group, giving the FETO group more significant survival chance. However, the true benefits of FETO are difficult to determine because the severity of $\mathrm{CDH}$ was not measured uniformly and there was great variability in the postnatal care of these infants. International, randomized controlled studies to evaluate the role of intrauterine fetal surgery in $\mathrm{CDH}$ cases with moderate and severe pulmonary hypoplasia is still needed [75].

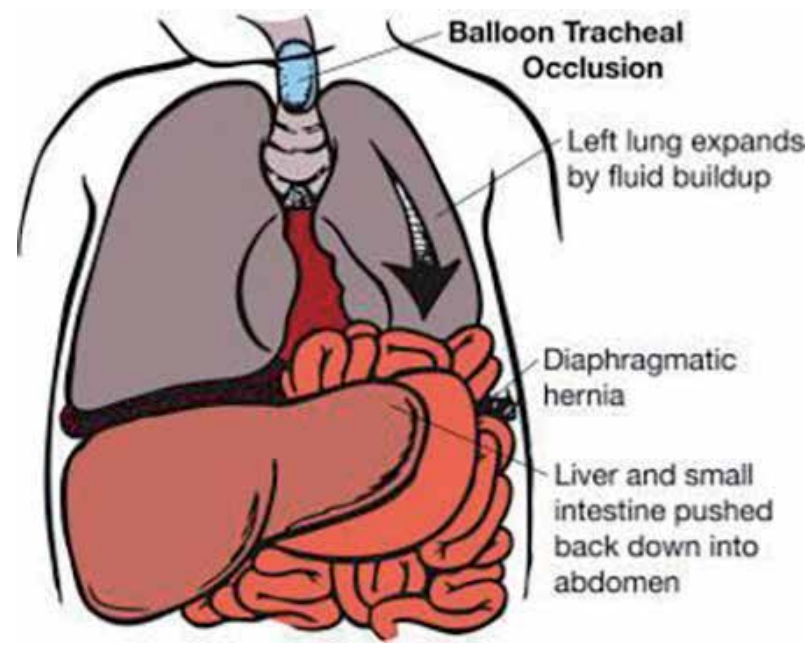

Figure 3.

Tracheal occlusion in fetus with congenital diaphragmatic hernia $(C D H)$ to increases lung volume, decreases herniation of abdominal viscera, and improves postnatal lung function. 


\subsection{Amniotic band syndrome (ABS)}

Amniotic band syndrome can lead to fetal death from umbilical cord strangulation and/or congenital limb deformity or loss, presumed to result from ischemia caused by constriction bands that interfere with vascular perfusion. There is increasing experience with intrauterine release of congenital constrictions and evidence is mounting that this therapy may help save/restore some limb function and morphology. The location of the bands and timing of fetal damage will affect the presentation, severity, and outcome of the condition. For example, pseudosyndactyly or limb amputation can be the results of constriction bands at the extremities, whereas more midline bands can result in craniofacial, thoracic, or abdominal defects, and may be fatal. The etiology of this syndrome is unknown, and theories range from a genetic basis or early disruption of the germinal disc to traumatic disruption of the membranes later in fetal development. Fetoscopic release of amniotic bands using minimally invasive surgery, can help in preservation of life and or limb saving in cases of ABS. The present acceptable functional outcome in $50 \%$ of cases is promising, although, clear selection criteria are needed to justify the risk of this in-utero invasive procedure, through increased experience and larger studies on this type of therapy for ABS (Figure 4).

In cases of extremity involvement by amniotic band syndrome, the band must be released using fetoscope to save the normal development of the limb and allow for normal limb function. Ultrasound imaging can easily diagnose the problem, showing; distal limb edema and interrupted blood flow by Doppler, with or without visualization of the constricting band. Although, the available reports include small case series, its results suggest that fetuses must have distal arterial limb flow detected by Doppler in order to benefit from intervention. Moreover, data from recent studies reported that fetuses with single limb involvement tend to do better than those with multiple involved limbs. Surprising, the incidence of PROM with this procedure seem to be higher than for other fetoscopic procedures, (reported rates up to $78 \%$ ). Although, small number of cases were reported in all available studies, and considering the learning curve in this studies, it could also be related to inherent membrane problems in these fetuses [76, 77].

\subsection{Sacrococcygeal teratoma (SCT)}

Although the mortality rate is 5\% for SCT diagnosed in the newborn, the mortality rate is about $50 \%$ for fetal SCT. Rupture of the tumor, or hemorrhage inside, or high output heart failure, and premature labor, form the main causes of fetal loss. Every attempt at interventions, to prevent this high prenatal mortality are the target of study of several fetal centers. Resection of the tumor (in utero) should be consider for treatment of pre-mature patients with early signs of heart failure or placentomegaly.

- Removal of the external part of the tumor is usually preferred, followed by later removal of its pelvic extension.

- Alternatively, radio-frequency, or thermal ablation can be used to occlude supplying arteries to the tumor.

It is important to note the related morbidity of all mentioned procedures, like risk of preterm delivery, beside procedure failure rate. The related morbidity can be reduced with minimally invasive procedures, however, the fear from decreased efficacy in local control, still a significant problem. Moreover, these less invasive 


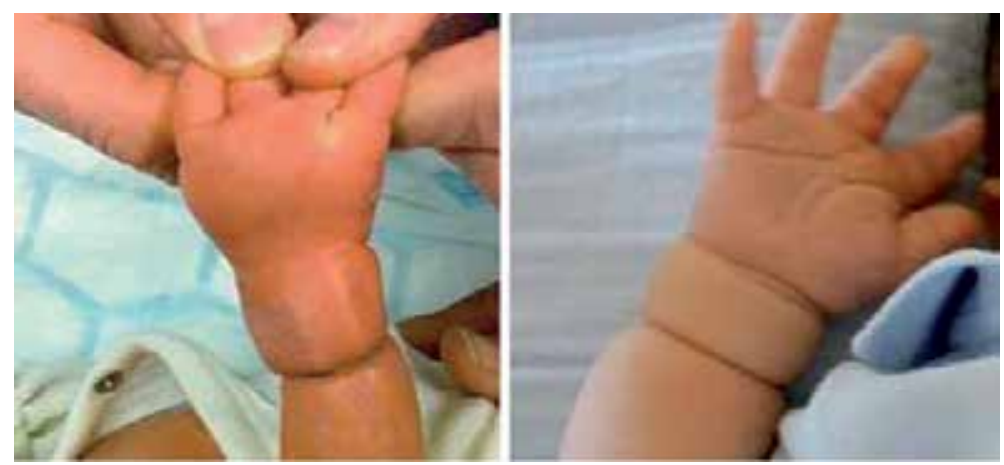

Figure 4 .

Some amniotic bands may constrict fetal limbs, and may be serious to cause limb amputation.

procedures should be used as early as possible, preferably before early signs of heart failure, to prevent IUFD. Certainly the best option is complete tumor resection, if possible. A systematic review on 34 cases of SCT from 1980 to 2013, using minimally invasive fetal procedures, they reported 44\% (14/32) overall survival with $29.7 \pm 4.0$ weeks, as mean gestational delivery age. They considered heart failure as a bad prognostic indicator, (30\% survival rate (6/20)). Another review compares two minimally invasive procedures, direct tumor control, and vascular occlusion of the tumor feeding vessels. Study included 33 cases, 11 cases submitted to vascular occlusion (group A) and 22 submitted to direct tumor control (group B). They reported $63.6 \%$ (7/11) survival in vascular ablation (group A) compared to $40.9 \%$ (9/22) in direct tumor control (group B). They claimed that reduction of the tumor blood supply slowly appears safer than rapid tumor necrosis which may lead to hemorrhage inside the tumor. Regarding outcomes of fetuses with large SCTs and fetal hydrops before viability, due to rarity of the tumor the available small case series suggest that fetal intervention does confer a survival advantage. However, randomized trials are needed to make a valuable conclusion, also long-term outcomes data are needed. Because these procedures are associated with significant risks, so to get the best benefit these patients must be performed only in specialized centers, and must be limited to cases presented with both high-output heart failure and fetal hydrops [78].

\subsection{Cystic pulmonary airway malformations (CPAM)}

Most prenatally detected lung lesions are cystic pulmonary airway malformations (CPAM), broncho-pulmonar sequestrations or so called 'hybrid' lesions, containing features of both. The outcome of most lesions are favorable even without pre-natal intervention, despite often impressive appearance at mid-gestation. During pregnancy many lesions may regress, or disappear completely. Therefore, non-operative treatment (watchful waiting) is preferred by most fetal surgeons. Surprisingly, pressure effect or hemo-dynamic changes may cause sudden physiologic derangements, which may end with progressive heart failure and intrauterine demise. Therefore, pre-natal intervention may be warranted to improve outcome. Pre-natal interventions for fetal lung lesions aim to alleviate the pressure effect of the mass by partial or complete removal of the lesion. Many surgical and non-surgical options have been reported.

- In macrocystic lesions, needle thoraco-centesis or thoraco-amniotic shunt drainage under ultrasound guided may be used for decompression. 
- In microcystic lesions, cysts are too small for drainage. In these cases, open fetal surgery has been performed.

- When a systemic feeding vessel is found, percutaneous laser coagulation or injection of a sclerosing agent can be successful.

Routine ultrasound used as screening method for detection of congenital lung lesions and require referral to a specialist center. Other co-existing problems of the fetus should be carefully evaluated to determine, the magnitude of related complications, delivery place, time and type, and if intra-uterine intervention is needed. Minimally invasive intra-uterine fetal intervention for severe lesions can greatly improve the prognosis of these fetuses. In a large study of thoraco-amniotic shunt placement for congenital lung mass or pleural effusion, performed on 75 fetuses at Children's Hospital of Philadelphia, they showed 55\% decrease in congenital cystic adenomatoid malformation volume and $27 \%$ of cases showed complete drainage of pleural effusion (73\% showed partial drainage of effusions) with hydrops resolution in $83 \%$ of fetuses (43/53), which was greatly correlated with survival. Survival to delivery was $93 \%$ (70/75), median gestational age was 36 weeks, with $68 \%$ (51/75) long-term survival rate. Fifty-six percent of fetuses were delivered at an average of 10 weeks after shunt placement. Duration of stay in the neonatal intensive care unit of 21 days, with for greater than 24 hours. This series affirms the survival benefit risk patients, but underscores the risks inherent to in utero intensive neonatal therapy required $[79,80]$.

\section{Future of fetal surgery}

Minimally invasive treatment is undoubtedly the future of fetal surgery, with the goal of providing the best possible outcome for the fetus, while minimizing the morbidity or mortality to the mother. So the concept of treating two patients at the same time is the challenging goal. To this end, significant efforts are being made toward safest methods for fetal intervention, particularly premature rupture of membranes. Currently a collaboration between University of California San Francisco, the University of California Berkeley, and Caltech is focusing on the development of a biocompatible adhesive (methyldihydroxyphenylalaninebased polymers) to preseal amniotic membranes before fetal surgery to prevent PPROM. This formula is currently under development and called "Amnioseal" which can be delivered just below the uterus to preseal the fetal membrane before amniotic access. At the moment, prenatal stem cell transplantation and gene therapy is under extensive research to treat a wide range of genetic conditions, and to extend the current application of fetal surgical intervention for only correction of structural fetal anomalies. The in utero stem cell transplantation will prevent the process of abnormal immune development before the fetus cellular differentiations. Two types of fetal stem cell therapy are currently under investigations for potential clinical use the in utero hematopoietic stem cell transplantation and mesenchymal stem cell transplantation. Many recent clinical trials of in utero hematopoietic stem cell transplantation reported that it has had a limited success in recipients without underlying immunodeficiency, however, some experimental data in a large animal model of intrauterine hematopoietic stem cell transplantation have demonstrated clinically relevant levels of chimerism, may be supporting its role for inherited hematologic disorders. On the other hand, the use of in utero human fetal mesenchymal stem cell transplantation has been reported for osteogenesis imperfect, although the preliminary results are promising, it temporally 
results. Finally, the studies of fetal gene therapy and gene-editing technology as a new treatment lines for fetal genetic disorders, are significantly advancing in the field of fetal therapy. However, the safety and long-term effect of these new types of treatment must be thoroughly evaluated in animal models before its applications in clinical practice.

\section{Author details}

Ahmed Abdelghaffar Helal

Pediatric Surgery Department, Faculty of Medicine, Al-Azhar University, Cairo, Egypt

*Address all correspondence to: helalhmada@azhar.edu.eg

\section{IntechOpen}

(C) 2019 The Author(s). Licensee IntechOpen. This chapter is distributed under the terms of the Creative Commons Attribution License (http://creativecommons.org/licenses/ by/3.0), which permits unrestricted use, distribution, and reproduction in any medium, provided the original work is properly cited. (cc) BY 


\section{References}

[1] Adzick NS. Fetal surgery for spina bifida: Past, present, future. Seminars in Pediatric Surgery. 2013;22:10-17

[2] Adzick NS, Thom EA, Spong $\mathrm{CY}$, et al. A randomized trial of prenatal versus postnatal repair of myelomeningocele. The New England Journal of Medicine. 2011;364:993e1004

[3] American College of Obstetricians and Gynecologists. Committee on ethics; American Academy of Pediatrics, committee on bioethics. Committee opinion no. 501: Maternalefetal intervention and fetal care centers. Obstetrics and Gynecology. 2011;118(Pt 1):405e10

[4] Bamforth JS. Amniotic band sequence: Streeter's hypothesis reexamined. American Journal of Medical Genetics. 1992;44:280-287

[5] Biffl WL, Spain DA, Rietsma AM, et al. Responsible development and application of surgical innovations: A position statement of the Society of University Surgeons. Journal of the American College of Surgeons. 2008;206:1204e9

[6] Chan JK, Gotherstrom C. Prenatal transplantation of mesenchymal stem cells to treat osteogenesis imperfecta. Frontiers in Pharmacology. 2014;5:223

[7] Chervenak FA, McCullough LB. An ethically justified framework for clinical investigation to benefit pregnant and fetal patients. The American Journal of Bioethics. 2011;11:39e49

[8] Chervenak FA, McCullough LB. The Professional Responsibility Model of Perinatal Ethics. Berlin: Walter de Gruyter; 2014

[9] Cignini P, Giorlandino C, Padula F, et al. Epidemiology and risk factors of amniotic band syndrome, or ADAM sequence. Journal of Prenatal Medicine. 2012;6:59-63

[10] Deprest JA, Flake AW, Gratacos $E$, et al. The making of fetal surgery. Prenatal Diagnosis. 2010;30:653-667

[11] Ehrenberg-Buchner S, Stapf AM, Berman DR, et al. Fetal lung lesions: Can we start to breathe easier? American Journal of Obstetrics and Gynecology. 2013;208:151.e1-151.e7

[12] Graves EC, Harrison RM, Padilla EB. Minimally invasive fetal surgery. Clinics in Perinatology. 2017;44(4):729-751

[13] Harrison MR, Adzick NS, Bullard $\mathrm{KM}$, et al. Correction of congenital diaphragmatic hernia in utero VII: A prospective trial. Journal of Pediatric Surgery. 1997;32:1637-1642

[14] Harrison MR, Adzick NS, Flake AW, et al. Correction of congenital diaphragmatic hernia in utero: VI. Hardearned lessons. Journal of Pediatric Surgery. 1993;28:1411-1417

[15] Jani J, Cannie M, Done E, et al. Relationship between lung area at ultrasound examination and lung volume assessment with magnetic resonance imaging in isolated congenital diaphragmatic hernia. Ultrasound in Obstetrics \& Gynecology. 2007b;30:855-860

[16] Jani J, Nicolaides KH, Gratacos $\mathrm{E}$, et al. Fetal lung-to-head ratio in the prediction of survival in severe left-sided diaphragmatic hernia treated by fetal endoscopic tracheal occlusion (FETO). American Journal of Obstetrics and Gynecology. 2006b;195:1646-1650

[17] Jani J, Nicolaides KH, Keller RL, et al. Observed to expected lung 
area to head circumference ratio in the prediction of survival in fetuses with isolated diaphragmatic hernia. Ultrasound in Obstetrics \& Gynecology. 2007a;30:67-71

[18] Jani JC, Nicolaides KH, Gratacos E, et al. Severe diaphragmatic hernia treated by fetal endoscopic tracheal occlusion. Ultrasound in Obstetrics \& Gynecology. 2009;34:304-310

[19] Javid P, Jaksic T, Skarsgard E, Lee S. Survival rate in congenital diaphragmatic hernia: The experience of the Canadian neonatal network. Journal of Pediatric Surgery. 2004;39:657-660

[20] Johnson MP, Corsi P, Badfield W, et al. Sequential urinalysis improves evaluation of fetal renal function in obstructive uropathy. American Journal of Obstetrics and Gynecology. 1995;173:59-65

[21] Johnson MP, on behalf of NAFTNet. The north American fetal therapy network (NAFTNet): A new approach to collaborative research in fetal diagnosis and therapy. Seminars in Fetal \& Neonatal Medicine.

2009;15:52-57

[22] Keller BA, Hirose S, Farmer DL. Fetal therapy. In: Milunsky A, editor. Genetic Disorders and the Fetus: Diagnosis, Prevention, and Treatment. 7th ed. Hoboken (NJ): John Wiley \& Sons, Inc.; 2016. pp. 989-1011

[23] Kunisaki S, Barnewolt C, Estroff J, et al. Ex utero intrapartum treatment with extracorporeal membrane oxygenation for severe congenital diaphragmatic hernia. Journal of Pediatric Surgery. 2007;42:98-106

[24] Kunisaki SM, Fuchs JR, Kaviani A, et al. Diaphragmatic repair through fetal tissue engineering: A comparison between mesenchymal amniocyte- and myoblast-based constructs. Journal of Pediatric Surgery. 2006;41:34-39
[25] Lam YH, Tang MHY, Shek

TWH. Thermocoagulation of fetal sacrococcygeal teratoma. Prenatal Diagnosis. 2002;22:99-101

[26] Lee H, Wagner AJ, Sy E, et al. Efficacy of radiofrequency ablation for twin-reversed arterial perfusion sequence. American Journal of Obstetrics and Gynecology. 2007;196:459.e1-459.e4

[27] Lenclen R, Ciarlo G, Paupe A, et al. Neurodevelopmental outcome at 2 years in children born preterm treated by amnioreduction or fetoscopic laser surgery for twin-to-twin transfusion syndrome: Comparison with dichorionic twins. American Journal of Obstetrics and Gynecology. 2009;201:291.e1-291.e5

[28] Lewi L, Jani J, Blickstein I, et al. The outcome of monochorionic diamniotic twin gestations in the era of invasive fetal therapy: A prospective cohort study. American Journal of Obstetrics and Gynecology. 2008;199:514.e1-514.e8

[29] Lewi L, Jani J, Cannie M, et al. Intertwin anastomoses in monochorionic placentas after fetoscopic laser coagulation for severe twin-to-twin transfusion syndrome: Is there more than meets the eye? American Journal of Obstetrics and Gynecology. 2006;194:790-795

[30] Liechty K. Ex utero intrapartum therapy. Seminars in Fetal \& Neonatal Medicine. 2010;15:34-39

[31] Liechty KW, Crombleholme TM, Flake AW, et al. Intrapartum airway management for giant fetal neck masses: The EXIT procedure (ex utero intrapartum treatment). American Journal of Obstetrics and Gynecology. 1997;177:870-874

[32] Liggins GC, Howie RN. A controlled trial of antepartum glucocorticoid treatment for prevention of the respiratory distress syndrome in 
premature infants. Pediatrics. 1972;50:515-525

[33] Liley AW. Intrauterine transfusion of fetus in haemolytic disease. British Medical Journal. 1963;2:1107-1109

[34] Louw JH, Barnard CN. Congenital intestinal atresia; observations on its origin. Lancet. 1955;269:1065-1067

[35] Luks FI, Deprest JA, Vandenberghe $\mathrm{K}$, et al. Fetoscopy-guided fetal endoscopy in a sheep model. Journal of the American College of Surgeons. 1994;178(6):609-612

[36] Luks FI, Deprest JA. Endoscopic fetal surgery: A new alternative? European Journal of Obstetrics, Gynecology, and Reproductive Biology. 1993;52:1-3

[37] Luks FI, Gilchrist BF, Jackson BT, Piasecki GJ. Endoscopic tracheal obstruction with an expanding device in a fetal lamb model:Preliminary considerations. Fetal Diagnosis and Therapy. 1996b;11:67-71

[38] Luks FI, Peers KH, Deprest JA, et al. The effect of open and endoscopic fetal surgery on uteroplacental oxygen delivery in the sheep. Journal of Pediatric Surgery. 1996a;31:310-314

[39] Luks FI, Wild YK, Piasecki GJ, De Paepe ME. Short-term tracheal occlusion corrects pulmonary vascular anomalies in the fetal lamb with diaphragmatic hernia. Surgery. 2000;128:266-272

[40] MacKenzie TC, David AL, Flake AW, et al. Consensus statement from the first international conference for in utero stem cell transplantation and gene therapy. Frontiers in Pharmacology. 2015;6:15

[41] MacKenzie TC. Fetal surgical conditions and the unraveling of maternal-fetal tolerance. Journal of Pediatric Surgery. 2016;51:197-199
[42] Makin EC, Hyett J, Ade-Ajayi N, et al. Outcome of antenatally diagnosed sacrococcygeal teratomas: Single-center experience (1993-2004). Journal of Pediatric Surgery. 2006;41:388-393

[43] Mallik AS, Fichter MA, Rieder S, et al. Fetoscopic closure of punctured fetal membranes with a cellular human amnion plugs in a rabbit model. Obstetrics and Gynecology. 2007;110:1121-1129

[44] Mann S, Johnson MP, Wilson RD. Fetal thoracic and bladder shunts. Seminars in Fetal \& Neonatal Medicine. 2010;15:28-33

[45] Manning FA, Harrison MR, Rodeck C. Catheter shunts for fetal hydronephrosis and hydrocephalus. Report of the international Fetal surgery registry. The New England Journal of Medicine. 1986;315:336-340

[46] McClain LE, Flake AW. In utero stem cell transplantation and gene therapy: Recent progress and the potential for clinical application. Best Practice \& Research. Clinical Obstetrics \& Gynaecology. 2016;31:88-98

[47] McCurdy CM, Childers JM, Seeds JW. Ligation of the umbilical cord of an acardiac-acephalus twin with an endoscopic intrauterine technique. Obstetrics and Gynecology. 1993;82:708-711

[48] McHugh K, Afaq A, Broderick N, et al. Tracheomegaly: A complication of fetal endoscopic tracheal occlusion in the treatment of congenital diaphragmatic hernia. Pediatric Radiology. 2010;40:674-680

[49] Mettauer NL, Pierce CM, Cassidy $\mathrm{JV}$, et al. One year survival in congenital diaphragmatic hernia, 1995-2006. Archives of Disease in Childhood. 2009;94:407

[50] Meuli M, Meuli-Simmen C, Yingling CD, et al. In utero repair of 
experimental myelomeningocele saves neurological function at birth. Journal of Pediatric Surgery. 1996;31:397-402

[51] Moise K. The history of fetal therapy. American Journal of Perinatology. 2014;31:557e66

[52] Senat MV, Deprest J, Boulvain $\mathrm{M}$, et al. A randomized trial of endoscopic laser surgery versus serial amnioreduction for severe twin-to-twin transfusion syndrome at midgestation. The New England Journal of Medicine. 2004;351:136-144

[53] Peranteau WH, Adzick NS, Boelig $\mathrm{MM}$, et al. Thoracoamniotic shunts for the management of fetal lung lesions and pleural effusions: A singleinstitution review and predictors of survival in 75 cases. Journal of Pediatric Surgery. 2015;50:301-305

[54] Peranteau WH, Boelig MM, Khalek $\mathrm{N}$, et al. Effect of single and multiple courses of maternal betamethasone on prenatal congenital lung lesion growth and fetal survival. Journal of Pediatric Surgery. 2016;51:28-32

[55] Roelofs LA, Eggink AJ, Hulsbergenvan de Kaa CA, et al. Fetal abdominal wall repair with a collagen biomatrix in an experimental sheep model for gastroschisis. Tissue Engineering. Part A. 2008a;14:2033-2040

[56] Roelofs LA, Eggink AJ, Hulsbergenvan de Kaa CA, et al. Fetal bladder wall regeneration with a collagen biomatrix and histological evaluation of bladder exstrophy in a fetal sheep model. Fetal Diagnosis and Therapy. 2008b;24:7-14

[57] Rosenkrantz JG, Simon RC, Carlisle JH. Fetal surgery in the pig with a review of other mammalian fetal techniques. Journal of Pediatric Surgery. 1968;3:392-397

[58] Roybal JL, Santore MT, Flake AW. Stem cell and genetic therapies for the fetus. Seminars in Fetal \& Neonatal Medicine. 2010;15:46-51

[59] Ruano R, Aubry MC, Barthe B, et al. Quantitative analysis of pulmonary vasculature by 3D-power Doppler ultrasonography in isolated congenital diaphragmatic hernia. American Journal of Obstetrics and Gynecology. 2006;195:1720-1728

[60] Ruano R, Duarte S, Bunduki V, et al. Fetal cystoscopy for severe lower urinary tract obstruction-Initial experience of a single Centre. Prenatal Diagnosis. 2010;30:30-39

[61] Ruano R, Sananes N, SangiHaghpeykar H, et al. Fetal intervention for severe lower urinary tract obstruction: A multicenter case-control study comparing fetal cystoscopy with vesicoamniotic shunting. Ultrasound in Obstetrics \& Gynecology. 2015;45:452-458

[62] Rychik J, Tian Z, Bebbington $M$, et al. The twin-twin transfusion syndrome: Spectrum of cardiovascular abnormality and development of a cardiovascular score to assess severity of disease. American Journal of Obstetrics and Gynecology. 2007;197:392.e1-392.e8

[63] Sala P, Prefumo F, Pastorino D, Buffi D, Gaggero CR, Foppiano M, et al. Fetal surgery: An overview. Obstetrical \& Gynecological Survey. 2014;69:218e28

[64] Sartoris J, Varnholt V, Dahlheim D, Schaible T. CDH in MannheimAlgorithm and results. Monatschr Kinderheillkd. 2006;153:717

[65] Sokol J, Shimizu Bohn D, et al. Fetal pulmonary artery diameter measurements as a predictor of morbidity in antenatally diagnosed congenital diaphragmatic hernia: A prospective study. American Journal of Obstetrics and Gynecology. 2006;195:470-477 
[66] Sroka M, Swiatokowska-Freund M, Golebiewski A, et al. Fetal surgery:

Future or way leading to nowhere. First experiences. Przeglad Pediatryczny. 2007;37:7-9

[67] Stege G, Fenton A, Jaffray B. Nihilism in the 1990s. The true mortality of CDH. Pediatrics. 2003;112:532-535

[68] Stirnemann JJ, Nasr B, Quarello $\mathrm{E}$, et al. A definition of selectivity in laser coagulation of chorionic plate anastomoses in twin-to-twin transfusion syndrome and its relationship to perinatal outcome. American Journal of Obstetrics and Gynecology. 2008;198:62.e1-62.e6

[69] Sviggum HP, Kodali BS. Maternal anesthesia for fetal surgery. Clinics in Perinatology. 2013;40:413-427

[70] Tsao K, Hawgood S, Vu L, et al. Resolution of hydrops fetalis in congenital cystic adenomatoid malformation after prenatal steroid therapy. Journal of Pediatric Surgery. 2003;38:508-510

[71] van der Wildt B, Luks FI, Steegers EA, et al. Absence of electrical uterine activity after endoscopic access for fetal surgery in the rhesus monkey. European Journal of Obstetrics, Gynecology, and Reproductive Biology. 1995;58:213-214

[72] Van Mieghem T, Klaritsch P, Dońe E, et al. Assessment of fetal cardiac function before and after therapy for twin-to-twin transfusion syndrome. American Journal of Obstetrics and Gynecology. 2009;200:400.e1-400.e7

[73] Van Peborgh P, Rambaud C, Ville Y. Effect of laser coagulation on placental vessels: Histological aspects. Fetal Diagnosis and Therapy. 1997;12:32-35

[74] Ville Y, Hyett J, Hecher K, Nicolaides K. Preliminary experience with endoscopic laser surgery for severe twin-twin transfusion syndrome. The New England Journal of Medicine. 1995;332:224-227

[75] Ville Y, Hyett JA, Vandenbussche F, Nicolaides KH. Endoscopic laser coagulation of umbilical cord vessels in twin reversed arterial perfusion sequence. Ultrasound in Obstetrics \& Gynecology. 1994;4:396-398

[76] Ville Y. Twin-to-twin transfusion syndrome: Time to forget the Quintero staging system? Ultrasound in Obstetrics \& Gynecology. 2007;30:924-927

[77] Welsh A, Agarwal S, Kumar S, et al. Fetal cystoscopy in the management of fetal obstructive uropathy: Experience in a single European Centre. Prenatal Diagnosis. 2003;23:1033-1041

[78] Westerburg B, Feldstein V, Sandberg $\mathrm{P}$, et al. Sonographic prognostic factors in fetuses with sacrococcygeal teratoma. Journal of Pediatric Surgery. 2000;35:322-326

[79] Clewell WH, Johnson ML, Meier PR, Newkirk JB, Zide SL, Hendee RW, et al. The New England Journal of Medicine. 1982;306:1320-1325

[80] Kohl T, Herin R, Heep A, et al. Percutaneous fetoscopic patch coverage of spina bifida aperta in the human-Early clinical experience and potential. Fetal Diagnosis and Therapy. 2006;21:185-193 
Section 2

Gastrointestinal Disorders 



\title{
Chapter 2
}

\section{Necrotizing Enterocolitis}

\author{
Rita Prasad Verma and Archana Kota
}

\begin{abstract}
Necrotizing enterocolitis (NEC) is the commonest inflammatory gastrointestinal disorder of newborn infants, occurring primarily in premature neonates. Presenting as a medical and surgical emergency, it is associated with significant morbidity and mortality. NEC is characterized by acute intestinal inflammation and necrosis with intramural dissection of gas, pathognomically appearing as pneumatosis intestinalis on radiography. The incidence and mortality, with an inverse relationship to maturation, range between $3-11 \%$ and $17-20 \%$ respectively. Mortality may be up to $50 \%$ in extremely premature infants who require surgery for intestinal perforation or gangrene. The exact etiopathogenesis is unknown. Over $90 \%$ of infants are premature and more than $98 \%$ are enterally fed. NEC presents with feeding intolerance and abdominal distension, which may rapidly progress to cardiorespiratory decompensation and death in severe cases. Intestinal dysbiosis and its functional and immunological immaturity are proposed to play roles in the pathogenesis. While exact triggers are undetermined, the disease is marked by an anomalous immunological response of enterocytes to inflammation, invoking cytokines and chemokines. NEC is treated with bowel rest, antibiotics, cardiorespiratory support, parenteral nutrition, and blood products transfusion. Approximately $30 \%$ of cases require surgery and a significant number of survivors suffer from neurological deficits, intestinal dysfunction, and post surgical short bowel syndrome.
\end{abstract}

Keywords: necrotizing enterocolitis, preterm infants, pneumatosis intestinalis, intestinal gangrene, intestinal perforation, intestinal dysbiosis, short bowel syndrome, feeding intolerance, heme positive stools, abdominal distension, cardiovascular decompensation

\section{Introduction}

Necrotizing enterocolitis (NEC) is an acquired, multifactorial and devastating gastrointestinal disease associated with high morbidity and mortality in preterm neonates. With an incidence of about $7 \%$ in infants with BW $<1500 \mathrm{~g}$ and mortality up to $30 \%$, NEC presents as a medical and surgical emergency $[1,2]$. It is characterized by ischemia, necrosis, and inflammation of bowel wall with invasion by gas-forming organisms and intramural dissection of gas, characteristically appearing as pneumatosis intestinalis in radiological and pathological studies. While exact etiology is undetermined, the pathogenesis is believed to be an anomalous innate immune response to an altered, less diverse intestinal microbiota by the highly immunoreactive enterocytes of premature infants, leading to inflammation and tissue necrosis $[3,4]$. The clinical presentation can be severe with cardiorespiratory collapse, shock, and disseminated intravascular coagulopathy (DIC), escalating 
to multisystem failure and death [2]. About one third of the cases require surgical intervention due to intestinal perforation and gangrene [5]. NEC is the commonest gastrointestinal (GI) disorder of preterm newborn infants, although term infants can be affected. NEC is associated with significant adverse outcomes, and approximately half of the survivors suffer from abnormal neurodevelopment independent of maturational status at birth. It is one of the most important causes of intestinal failure in children. Despite substantial advances in its diagnosis, prevention, and management strategies, the incidence has not changed, especially in very low birth weight neonates, and the morbidity and mortality associated with necrotizing enterocolitis continue to be high.

\section{Epidemiology}

NEC constitutes about 2-5\% of all NICU admissions. The incidence reported in 2012 by the Canadian Neonatal Network (CNN) in infants less than 33 weeks of gestational age (GA) was $5.1 \%$ [1]. In the United States the incidence is estimated to be $1-3$ per 1000 live births $[2,6]$, while its prevalence is $0.3-2.4$ per 1000 live births. There is considerable variability in incidence among different geographical locations and neonatal intensive care units [6-8]. Henry and Moss noted an overall incidence of 3-7\% in 2005 [5]. A review by the National Institute of Child Health and Human Development (NICHD) Neonatal Research Network data from 1998 to 2001 reported a 7\% incidence of NEC among very low birth weight infants [9], while a more recent report from the network in 2010 documented an incidence of $11-15 \%$ in neonates $<1500 \mathrm{~g}$ or $<32$ weeks at birth [10]. The incidence was found to be relatively unchanged at approximately $7 \%$ in infants weighing $<1500 \mathrm{~g}$ in another report published in 2011 [11]. Ninety percent of the infants are preterm and the rest term or late preterm. Incidence and mortality are inversely related to GA and birth weight (BW). Mortality in preterm infants from NEC may be up to $30-50 \%$, and $27 \%$ of infants require surgical intervention with an overall case fatality rate of $15 \%[5,11,12]$. The mortality rate is higher in surgical NEC and African American males [13]. Forty-six percent of survivors suffer from abnormal neurodevelopment, and $12 \%$ of all cases of GI failure in children are due to NEC [14]. The risk and mortality associated with NEC were stratified according to BW and GA in a cohort of extremely premature infants in who the overall incidence was estimated to be $7.5 \%$ (Tables 1 and 2) [2].

Despite the variability in incidence among studies with rates ranging between 3 and 15\% in VLBW infants, a relative stability in the incidence over time has been noted. Survival in NEC has not changed in the past five decades, the average mortality being $20-30 \%$ and up to $50 \%$ in infants requiring surgical management [10]. The proportion of neonates with NEC requiring surgical intervention has

\begin{tabular}{|l|l|l|}
\hline Birth weight (grams) & Risk of NEC(\%) & Mortality with NEC ( \%) \\
\hline $501-750$ & 12 & 42 \\
\hline $751-1000$ & 9 & 29 \\
\hline $1001-1250$ & 6 & 21 \\
\hline $1251-1500$ & 3 & 16 \\
\hline
\end{tabular}

Table 1.

Risk and mortality associated with NEC based on birth weight. 


\begin{tabular}{|l|l|}
\hline $\begin{array}{l}\text { Gestational Age } \\
\text { (in weeks) }\end{array}$ & $\begin{array}{l}\text { Risk of NEC } \\
\text { (percent \%) }\end{array}$ \\
\hline 22 & 11 \\
\hline 23 & 16 \\
\hline 24 & 11 \\
\hline 25 & 9 \\
\hline 26 & 10 \\
\hline 27 & 8 \\
\hline 28 & 8 \\
\hline
\end{tabular}

Table 2.

Risk of NEC based on gestational age.

also remained stable at approximately 30\% [15]. The reasons for such observations are the decreasing gestational age limit for neonatal viability and increased survival of extremely premature infants with advances in neonatal care. Practice implementations, such as standardizing enteral feeding guidelines, exclusive feedings of own mother's milk, using donor breast milk when mother's milk is not available, minimizing duration of empiric antibiotics after birth, and avoiding packed red blood cells (PRBC) transfusions as well as antacid use in preterm infants, are associated with a decrease in incidence of NEC in very low birth weight infants [16].

\section{Etiology and risk factors}

The exact etiology of NEC is undetermined and multiple risk factors have been forwarded. NEC occurs in a stereotypic relation at chronological age of onset to the gestational age at birth, the younger the gestation, the later the onset; and requires that the infant be fed $[1,11]$. In one study the median age at onset in infants with a GA of less than 26 weeks was 23 days compared to a median age of 11 days for more mature infants with a GA of greater than 31 weeks [17]. Prematurity is the single greatest risk factor with almost $90 \%$ patients being premature. Enteral feeding is the second most common feature with over $98 \%$ of cases having a history of feeding. However, rate of advancement unless excessive, trophic, and early versus late and colostrum feeding are not conclusively proven to have any effect on the occurrence of NEC [18]. Other suggested risk factors are the $5 \mathrm{~min}$ Apgar score $<7$, outborn status, body temperature $0 \mathrm{f} 36^{\circ} \mathrm{C}$ at $1 \mathrm{~h}$ of age, cesarean section, use of indomethacin with or without dexamethasone, sepsis, use of inotropes, severe metabolic acidosis, patent ductus arteriosus (PDA), gastroschisis, severe anemia, polycythemia, packed red blood cell (PRBC) transfusion, use of $\mathrm{H} 2$ antagonist, exposure to empirical antimicrobials, and black and Hispanic ethnicity [19]. Approximately $10 \%$ of cases occur in term and late preterm infants. Risk factors for NEC in term infants are nonhuman milk feeding; preexisting illnesses, such as congenital heart disease; primary gastrointestinal disorders; sepsis; polycythemia; respiratory disease; hypotension; neonatal 
abstinence; fetal growth restriction; and perinatal hypoxia [20]. Despite the fact that no predilection for sex, race, or ethnicity has been conclusively established, a higher incidence is observed in male African American infants than in any other single demographic. This could be related to the higher incidence of prematurity in this ethnic group than in the general US population. Hypoxic ischemic injury is no longer considered a major predisposing factor in the development of NEC except in term babies [21].

\section{Pathology}

NEC primarily affects the ileum and colon, the commonest location being ileocecal area [22]. The entire gastrointestinal tract may be involved in severe cases. On gross examination, the bowel loops are distended with areas of hemorrhage, congestion, necrosis, and pneumatosis (Figure 1). On microscopic examination, signs of inflammation, mucosal edema, bacterial invasion, submucosal and intramural gas bubbles, and ischemic transmural necrosis are seen. Intestinal perforation may happen when the entire thickness of bowel is involved leading to pneumoperitoneum, peritonitis, and portal venous gas (Figure 2). Microscopically, the predominant feature is coagulation necrosis, suggesting an ischemic origin of NEC. The aggregated inflammatory cells are both acute and chronic, such as neutrophils, lymphocytes, and macrophages representing an appropriate response to pathogenic bacterial invasion and tissue necrosis. Epithelial regeneration, granulation tissue formation and fibrosis may be seen suggesting reparative histological process [23]. Common pathogens isolated in NEC are Enterobacteriaceae including Escherichia, Salmonella, Enterobacter, and Klebsiella (68\%); staphylococcal species (26\%); clostridium species (4\%); viruses including rota, echo, corona, and toro (11\%); and candida (1\%). No organism is isolated in $3 \%$ of cases.

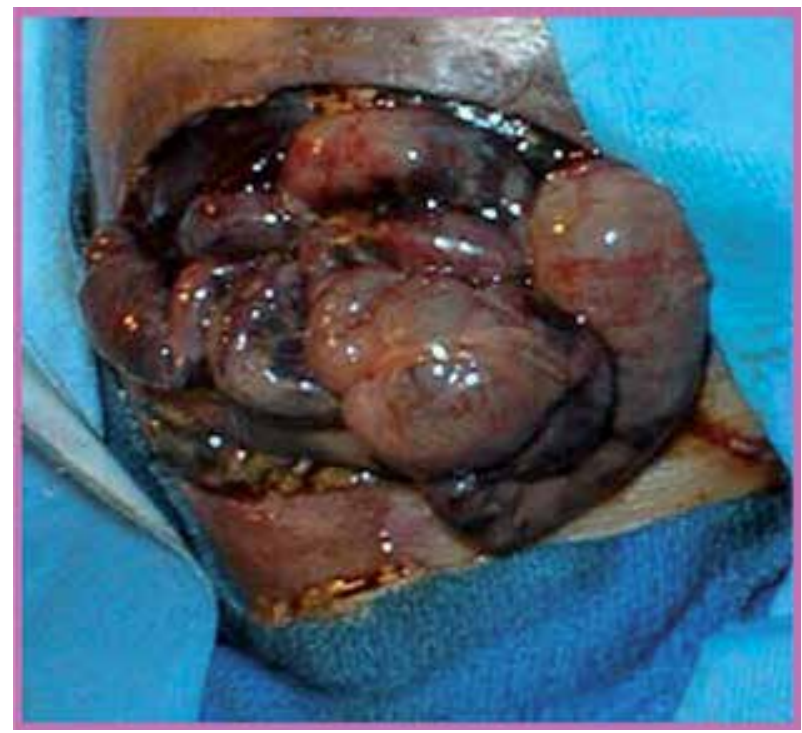

Figure 1.

Macroscopic appearance of necrotizing enterocolitis showing necrotic bowel loops. [Courtesy of Renu Sharma, $M D$, Professor of Pediatrics, University of Florida at Jacksonville, USA]. 


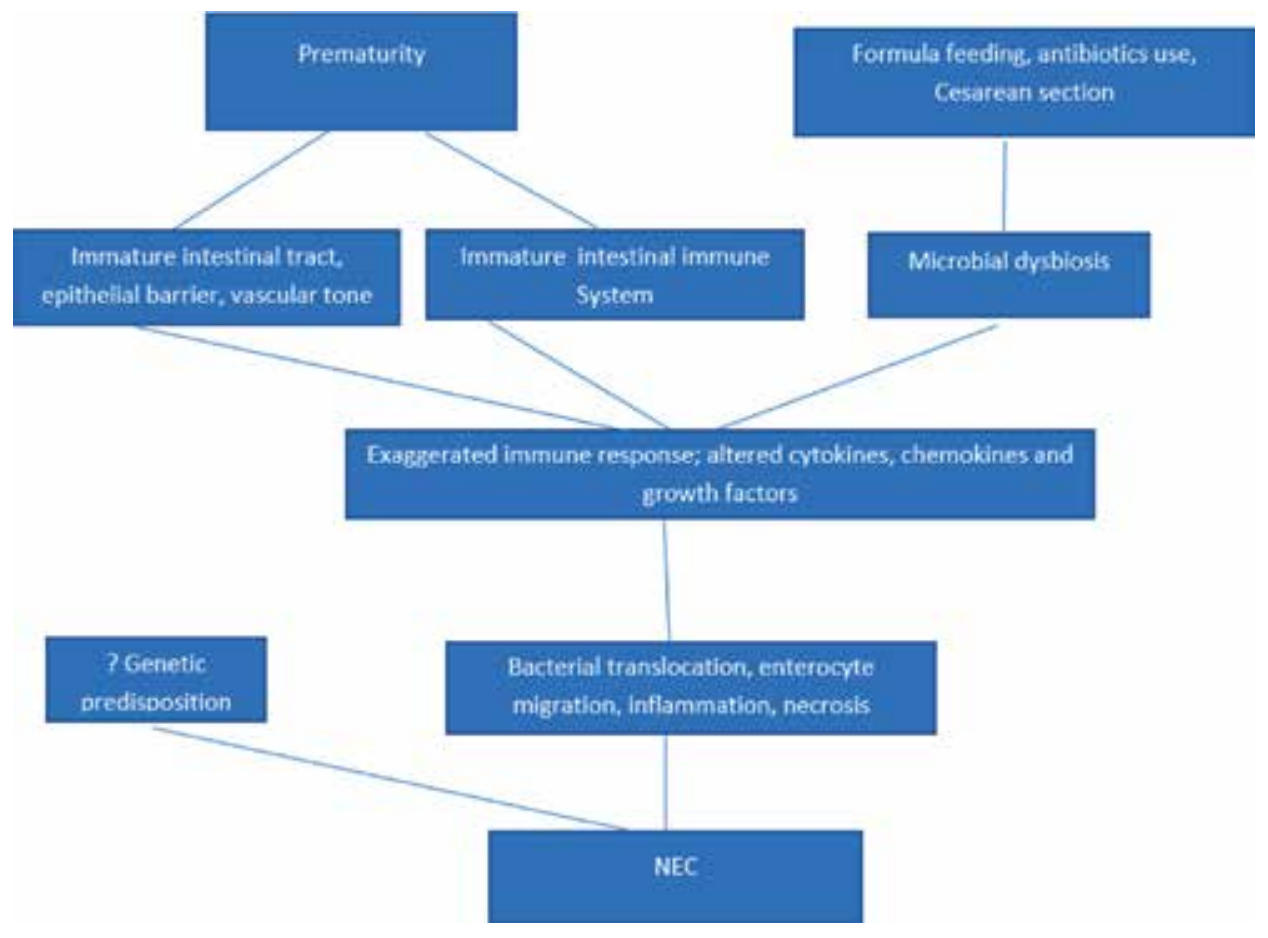

Figure 2.

Schematic presentation of pathogenesis of NEC.

\section{Pathogenesis}

The pathogenesis of NEC is complex, multifactorial, and incompletely defined. The disorder is believed to be a composite result of intestinal immaturity, aberrant immunological response, and gut microbial dysbiosis [3, 4, 23, 24]. It almost never occurs when the intestinal immune system is mature and intact, even when other risk factors are present. Experimental NEC does not occur in sterile environment. Research support the hypothesis that NEC in the preterm infant results from a multifactorial process that requires the concurrent presence of an immature intestinal tract and immune system leading to increased susceptibility, factors causing disruption of the normal intestinal bacterial microbiome with growth of potentially pathogenic bacteria, and an exaggerated inflammatory host response with the release of cytokines and chemokines (Figure 2). A genetically determined predisposition to necrotizing enterocolitis has been also proposed implying the contribution of genetic polymorphisms in the pro-inflammatory cytokines associated with NEC [25]. The combination of compromised intestinal epithelial barrier; underdeveloped and anomalous immune defense; abnormal mesenteric vascular development, tone, and flow; and altered luminal microbiota shaped by formula feedings, antibiotic exposure, and cesarean delivery presumably leads to intestinal inflammation and gangrene. NEC is triggered when several risk factors heighten neonatal intestinal inflammation and an irreversible borderline is surpassed.

\subsection{Intestinal immaturity, dysbiosis, and barrier dysfunction}

Immaturity of neonatal intestinal mucosal barrier and mucosal immune system is characterized by decreased mucus coat, altered mucus protein, reduced $\operatorname{Ig} \mathrm{A}$, and abnormal epithelial membrane and tight junctions [4, 26, 27]. Preterm intestinal 
mucosa is highly immunoreactive, and fetal human enterocytes have been shown to evoke excessive immunological and inflammatory response compared to adults. An imbalance between epithelial cell injury and repair leads to a gut barrier failure and a consequent cycle of bacterial invasion, immune activation, uncontrolled inflammation, and gut necrosis (Figure 2).

\subsection{Feeding and immature GI function}

Premature GIT is relatively deficient in digestive functions and peristaltic motility in addition to immune responses [28]. Dysfunctional gastric emptying and increased gastric $\mathrm{pH}$ add to gut barrier disruption and epithelial permeability [4, 29-31]. Aggressive feeding with peristaltic dysmotility leads to stasis of intraluminal contents and intestinal dilatation, which may further impair epithelial barrier (EB). These, in concurrence with microbial dysbiosis, result in abnormal signal transduction across the EB with consequent inflammation, apoptosis, and necrosis. The balance between the pro-inflammatory and anti-inflammatory signaling is affected with an inappropriate response to pathogenic microorganisms.

\subsection{Role of cytokines and chemokines}

NEC is associated with increased expression of inflammatory cytokines, such as tumor necrosis factor (TNF), interleukin (IL) - $1 \beta$, IL-6, IL-8/CXC-motif ligand 8 (CXCL8), IL 10 monocyte chemoattractant protein-1/CC-motif ligand (CCL)-2, macrophage inflammatory protein-1 $\beta / C C L 3$, and C-reactive protein in plasma and affected tissues [32]. These cytokines can disrupt the epithelial barrier and augment intestinal injury. Serum levels of cytokines/chemokines are elevated in NEC, and increased TLR4 and abnormal IkB/NFkB suggest excessive abnormal immunological response [33-35]. Lower blood TGF- $\beta$ and interleukin (IL)-2 and higher IL-8 levels are found in ELBW infants with NEC. A developmental immaturity is noted in IkB expression, the molecule that inhibits cytokines activation via NFkB in NEC. Recently the role of toll-like receptor 4 (TLR4) signaling in the pathogenesis of NEC has been highlighted. Hypoxia, infection, and prematurity accentuate the expression of TLR4 in the intestinal mucosa. TLR4 is subsequently activated by enteric bacteria, triggering an inflammatory cascade which results in increased gut mucosal injury and reduced epithelial repair. Activation of cytoplasmic innate immune receptors, NOD2 and TLR9 leads to inhibition of TLR4, with restoration of the intestinal epithelial barrier and reduction in severity of NEC in experimental models. Other factors implicated in pathogenesis of NEC are platelet-activating factor, nitric oxide, reactive oxygen species, and transforming growth factors. However, despite success in animal model systems, no significant improvement in treatment and outcomes of NEC has been achieved due to an incomplete understanding of the developing immune system in premature infants and inability to replicate them in animal models [32].

\section{Clinical presentation}

NEC presents acutely with feeding intolerance, heme-positive stools, abdominal distension, gastric residuals, and vomiting in a previously stable and feeding preterm infant. Commonly associated nonspecific symptoms are temperature instability, apnea, bradycardia, oxygen desaturation, and lethargy. There may be abdominal wall erythema, abdominal tenderness, and decreased or absent bowel sounds (Figure 3). As the disease process advances, cardiorespiratory 


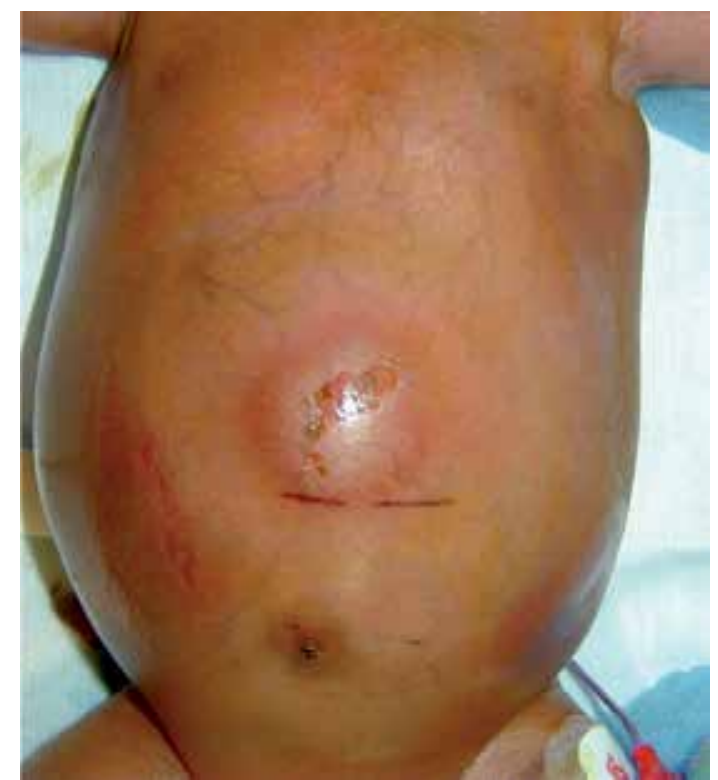

Figure 3.

Abdominal distension, erythema and skin ulceration in a case of necrotizing enterocolitis. [Courtesy of Renu Sharma, MD, Professor of Pediatrics, University of Florida at Jacksonville, USA].

decompensation, septic shock, and multi-organ failure may supervene. The diagnosis is confirmed by the presence of pneumatosis intestinalis in abdominal $\mathrm{X}$-ray which is pathognomonic of NEC (Figure 4). The course may be mild to moderate with recovery with antibiotics, GI rest, and correction of biochemical and hematological anomalies, or fulminant with early signs of severe systemic

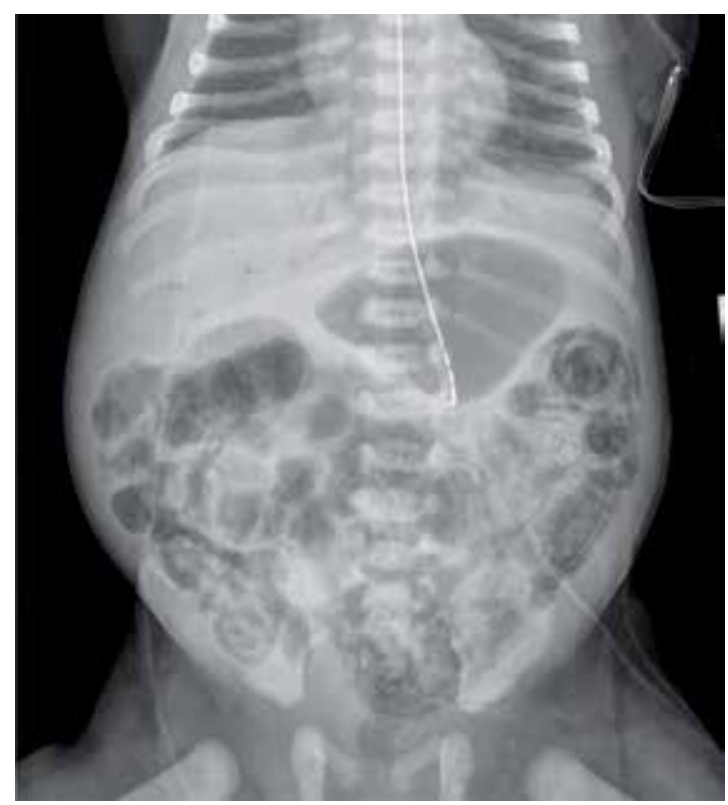

Figure 4.

Extensive pneumatosis with branching linear lucencies in the liver, consistent with portal venous gas. [Courtesy of Dr. Renu Aggarwal, Attending Neonatologist, NYU Winthrop Hospital, Mineola, NY, USA]. 


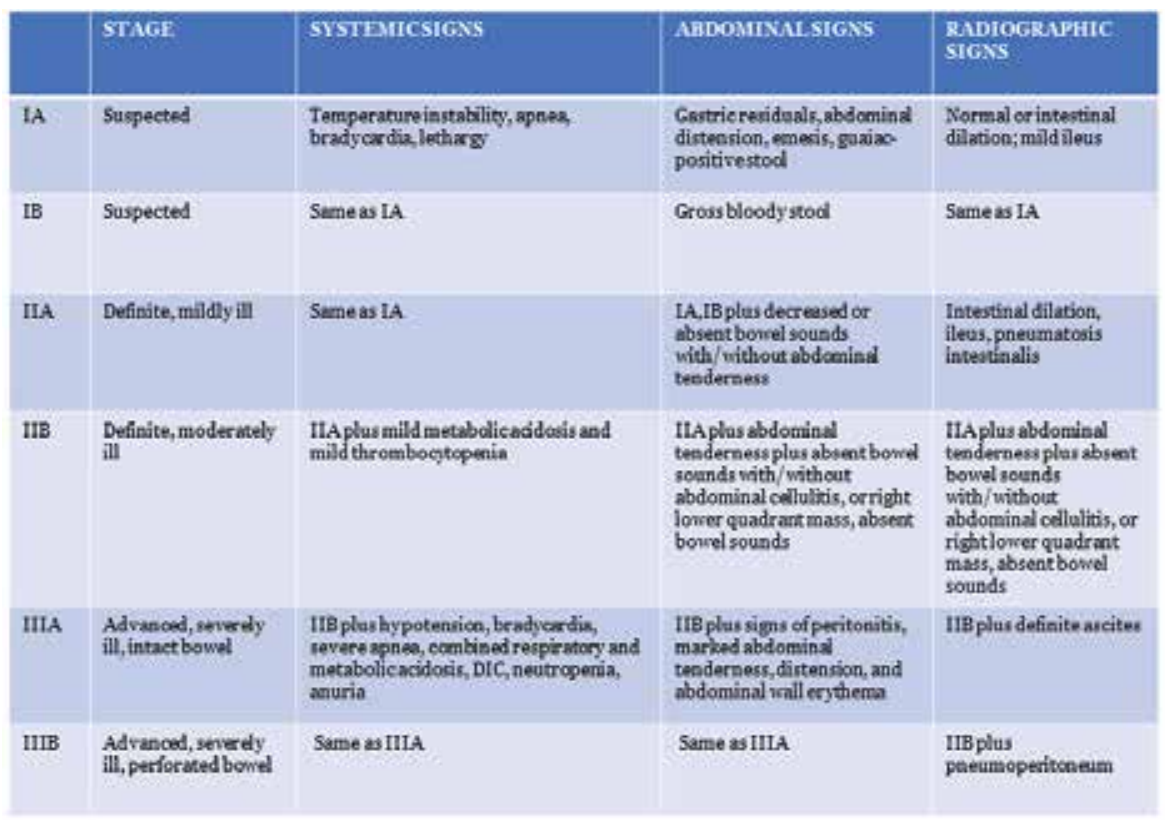

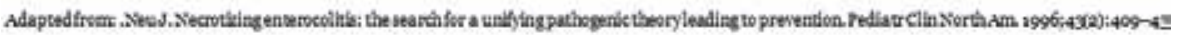

Table 3.

Modified Bell's staging criteria for necrotizing enterocolitis in neonates [33].

inflammatory response and poor response to correction of metabolic and hematological derangements, such as severe metabolic acidosis, hyponatremia, hyperglycemia, thrombocytopenia, DIC, anemia, and neutropenia, eventually progressing to death.

Age of onset varies in an inverse relationship with GA at birth, and the average post menstrual gestational age of NEC is estimated to be 31-32 weeks. In a cohort of preterm infants under 33 weeks gestational age, NEC presented at a mean of 7 days in more mature infants, while it was delayed to 32 days in lower birth weight and gestational age neonates [1]. The average age of onset has been reported to be 20.2 days for babies born at less than 30 weeks' estimated gestational age, 13.8 days for babies born at 31-33 weeks, and 5.4 days for babies born after 34 weeks of gestation. Term infants develop necrotizing enterocolitis much earlier, with the average age of onset within the first week or within the first 1-2 days of life [36].

To classify the severity of NEC based on clinical findings, a staging criterion was proposed by Bell in 1978 which was later modified (Table 3) [37]. In about one third of cases, NEC is suspected but not confirmed (stage I), and symptoms resolve gradually with treatment. In $25-40 \%$ of cases, the progression of NEC is fulminant with signs of peritonitis and sepsis and the rapid development of DIC and shock (stage III). About $30 \%$ of the cases may develop intestinal perforation, peritonitis, and other complications necessitating surgical intervention. Mean LOS is 62 days in surgical and 36 days in medical NEC cases [15]. Surgical NEC cases incur higher hospital costs.

\section{Laboratory and radiological investigations}

In all cases of NEC, CBC with diff, blood culture, C-reactive protein, serum electrolytes, $\mathrm{pH}$, lactate, acid-base indicators, arterial blood gases, 
and pertinent radiography should be done. The characteristic anomalies are metabolic or mixed acidosis, high C-reactive protein (CRP), hyponatremia, hyperglycemia, thrombocytopenia, neutropenia, or leukocytosis with high I/T ratio. CSF studies are suggested, and peritoneal fluid analysis for bacteria and fecal material should be done if paracentesis abdominis is performed for therapeutic or diagnostic purposes. Presentation of NEC is similar to, or may be associated with sepsis, and the differentiation is confirmed by the presence of pneumatosis intestinalis (PI) on radiography (Figure 5). Apart from PI other radiological features of NEC are ileus, bowel wall thickness, and bowel perforation with peritoneal air. Bowel wall thickening, with or without echogenicity, indicates increasing inflammation, swelling, and perfusion of the area. Bowel loops may be separated by the presence of peritoneal fluid and give an impression of thickening. Thin bowel wall with a central echogenic focus and a hypoechoic rim, called pseudo-kidney sign, if present, may indicate necrotic bowel and imminent perforation. Ultrasound detection of small air bubbles in the bowel wall as in pneumatosis intestinalis can be spatially differentiated from air bubbles in stool that can sometimes be misdiagnosed as pneumatosis on radiographs. Ultrasonography also can detect intermittent gas bubbles in the liver parenchyma and portal venous system that are not detected on radiography. Ultrasound is more sensitive in detecting peritoneal fluid collections. Doppler ultrasound is dynamic and permits real-time visualization of bowel wall thickness, peristalsis, and perfusion. It is more sensitive than abdominal radiography in detecting bowel necrosis [15]. Evidence of free peritoneal air and ascites indicate intestinal perforation. Contrast enemas are not recommended if NEC is suspected, as it may result in bowel perforation with extravasation of contrast material into the peritoneum. Near-infrared spectroscopy (NIRS) is a new, noninvasive method of estimating local tissue hemoglobin oxygen saturation by measuring the difference between oxyhemoglobin and deoxyhemoglobin and may have utility in diagnosing intestinal ischemia in NEC. Fortune et al. demonstrated cerebro-splanchnic oxygenation ratio $<0.75$ to have a positive predictive value of 0.75 for intestinal ischemia, whereas, if above 0.75 , intestinal ischemia is excluded with a negative predictive value of 0.96 [38].

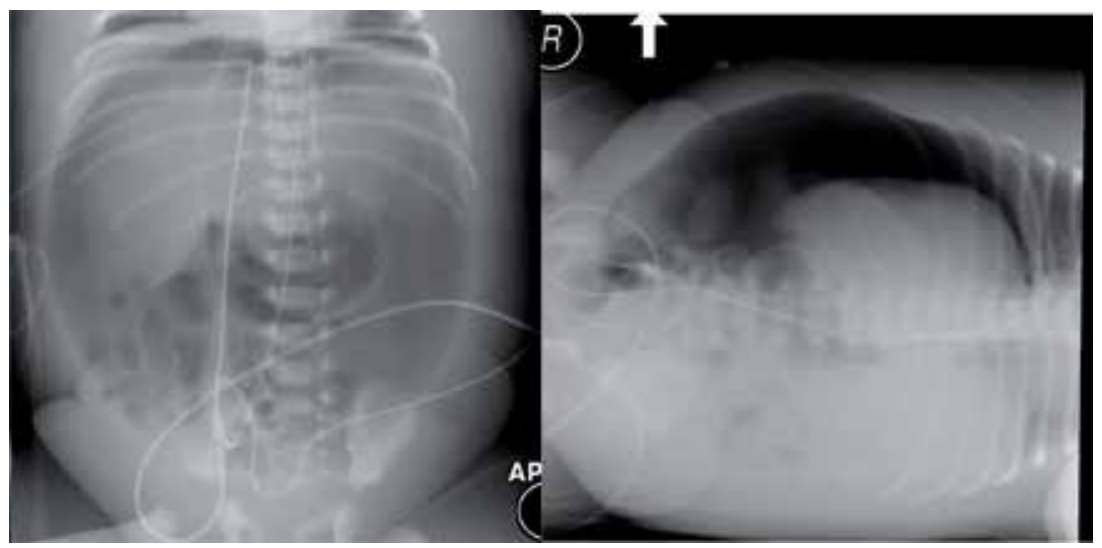

Figure 5.

On left: massive pneumoperitoneum with visualization of falciform ligament, massive lucency involving the entire abdomen, visualization of the liver margin. On right: left lateral decubitus radiograph demonstrating massive lucency with visualization of the liver margin and bowel. [Courtesy of Dr. Renu Aggarwal, Attending Neonatologist, NYU Winthrop Hospital, Mineola, NY, USA]. 


\subsection{Differentiating medical and surgical NEC: use of biomarkers}

Pneumoperitoneum is not a very reliable clinical feature for surgical NEC and is observed in less than half of all infants with intestinal perforation or necrosis $[15,39]$. Clinical deterioration despite maximal medical therapy is considered a relative indication for surgical intervention. Research has been done to identify a dependable predictor for intestinal necrosis. The most commonly used biochemical markers for bowel necrosis among pediatric surgeons are platelet count (99\%), C-reactive protein (CRP) concentration (90\%), white blood cell count (83\%), lactate levels (43\%), fecal calprotectin 10\%, and interleukin (IL)-6 or interleukin-8 10\% [40]. Fecal calprotectin is a marker of intestinal inflammation and can differentiate between local Bell stage II and systemic Bell III NEC with 76\% sensitivity and 92\% specificity [41]. Fecal levels of another protein, S100A12, are noted to be higher in infants with suspected NEC who subsequently develop bowel perforation. Unremitting and relentlessly high CRP levels despite treatment may indicate advanced stage of NEC and bowel necrosis. IL-8 levels have been shown to be significantly elevated in patients developing surgical NEC compared to medically managed NEC [42]. The levels can also discriminate NEC totalis from focal and multifocal diseases and predict 60-day mortality [43]. Maximum concentration of CRP and duration of CRP elevation are increased in infants who developed intestinal strictures following NEC, while the negative predictive value of CRP levels $<10 \mathrm{mg} / \mathrm{dL}$ for stricture development is $100 \%$ [44]. Intestinal fatty acid-binding protein (I-FABP), a marker of intestinal injury and progression to severe NEC, is located in mature enterocytes of small intestinal villi and is released into the blood stream after cell disruption and subsequently excreted into the urine. At onset of symptoms, I-FABP concentrations have been shown to be significantly higher in infants who later developed surgical NEC [45]. Other biomarkers being investigated for surgical NEC are serum amyloid A protein, liver fatty acid-binding protein, urine peptides, and heart rate characteristic index.

\section{Differential diagnosis}

Blood stream infection can present like NEC and must be ruled out. Sepsis and other conditions that can cause feeding intolerance, rectal bleeding, abdominal distension, gastric retention of feed, or intestinal perforation can be differentiated from NEC by the absence of radiologic evidence of pneumatosis intestinalis and the characteristic combination of rectal bleeding presenting as heme-positive or grossly bloody stools, abdominal distention, bilious vomiting, and gastric aspirates as seen in NEC. Spontaneous intestinal perforation is characterized by a single noninflammatory perforation that is typically located at the terminal ileum or colon. It occurs primarily in infants with birth weight $<1000 \mathrm{~g}$ and is differentiated from NEC by the presence of less severe systemic signs and absence of pneumatosis intestinalis. Infectious enteritis may present with frequent, occasionally bloody stools with abdominal distension but no pneumatosis. Congenital anomalies of GIT, such as Hirschsprung disease, small bowel atresia, meconium ileus, and acquired conditions like volvulus and intussusception, present with intestinal obstruction and at times secondary enterocolitis. Abdominal radiography differentiates these conditions from NEC. Anal fissures can result in rectal bleeding and can be detected on pertinent thorough physical examination. Milk protein allergy-induced enterocolitis may present with heme-positive stools and other GI symptoms similar to NEC in preterm infants but no pneumatosis. Such patients respond to dietary modification by switching the formula to extensively hydrolyzed or amino acid-based ones and may have eosinophilia along with thrombocytosis. 


\section{Management}

The basic principles of management of NEC are bowel decompression and rest, antibiotics coverage, cardiorespiratory support, fluid resuscitation, provision of blood products, and surgical intervention if indicated. The management strategies according to Bell's staging are outlined in Table 4. Surgical consultation is obtained in all stages of NEC including stage 1. Total parenteral nutrition (TPN) should be provided during the period that the infant is nil by mouth.

\subsection{Medical management}

The principles are as follows: (1) bowel decompression and rest, (2) parenteral hydration and nutrition, (3) respiratory and cardiovascular support, (4) antibiotic therapy, (5) general supportive care, (6) fluid resuscitation, and (7) serial close laboratory monitoring and radiologic surveillance. The focus is on limiting the progression of the disease. Intermittent or continuous nasogastric suction is done for bowel decompression, TPN is provided to ensure nutrition, and fluid is replaced to correct third space losses. Adequate cardiorespiratory support is of paramount value, and hematologic anomalies, such as DIC, anemia, and thrombocytopenia, are promptly corrected. Metabolic abnormalities, such as metabolic acidosis, hyponatremia, and hyper- or hypoglycemia, are appropriately treated. Even though an infectious agent has not been identified or attributed to NEC, antibiotics are routinely used in its treatment. Observational data reveal that 20-30\% cases of NEC have bacteremia, and pathogenic bacteria are recovered from pathologic specimens and peritoneal fluid. Epidemic outbreaks of NEC are common, and the clinical picture improves with antibiotics. The efficacy of antibiotic agents is documented in experimental animal models for NEC. The commonly used empiric broad-spectrum antibiotic combinations are as follows: ampicillin gentamicin (or amikacin), ampicillin, gentamicin (or amikacin) and clindamycin or ampicillin, cefotaxime, and metronidazole. Ceftazidime is an alternative choice for cefotaxime. Other antibiotic combinations are tazobactam and gentamicin (or amikacin); vancomycin, piperacillin-tazobactam, and gentamicin; and meropenem and vancomycin if methicillin-resistant staphylococcus or ampicillin-resistant enterococcus infections are suspected. Amikacin may be used in centers with significant gentamicin resistance. Metronidazole or clindamycin is added to cover anaerobic bacteria, especially in cases where infant is fed orally before NEC supervenes.

Evaluation of progression of the disease is important in order to take appropriate and timely steps to avoid further damage to the bowel. Serial laboratory monitoring is routinely performed. At diagnosis stool for guaiac test, complete blood and differential neutrophil counts, blood culture, CSF study if indicated, C-reactive protein, platelet count, serum electrolytes, $\mathrm{pH}$, creatinine, blood urea nitrogen, and acid-base studies are obtained and monitored q 12 or $24 \mathrm{~h}$ or more frequently

\begin{tabular}{ll}
\hline $\begin{array}{l}\text { NEC Bell's } \\
\text { stage }\end{array}$ & Treatment \\
\hline 1a and $1 \mathrm{~b}$ & Antibiotics $\times 3$ days, NPO \\
\hline $2 \mathrm{a}$ & NPO, antibiotics $\times$ 7-10 days \\
\hline $2 \mathrm{~b}$ & NPO, antibiotics $\times 14$ days \\
\hline $3 \mathrm{a}$ & As in 2b plus, fluid resuscitation, inotropic and ventilator support, blood products \\
\hline $3 \mathrm{~b}$ & As in 3a plus surgery \\
\hline
\end{tabular}

Table 4.

Management principles of NEC [65]. 
if needed. In addition, arterial blood gas values are measured and repeated every 4-6-12 $\mathrm{h}$ as per the severity of respiratory decompensation. Serial lactate levels are helpful in monitoring progression of the necrotic process and assessing systemic status. Worsening or persistent metabolic acidosis and persistent hyperglycemia or thrombocytopenia are poor prognostic signs. Improvement in metabolic acidosis is a positive prognostic sign but may be misleading if blood circulation to the necrotic bowel is completely severed and the generated lactic acid cannot enter the circulation. Blood in stools is not predictive of resolution or outcome. Radiographic monitoring is done with abdominal radiograph performed in supine position during the initial phase of illness. A lateral decubitus view is simultaneously obtained with the infant's left side down to visualize the presence of free air over the liver. It should be repeated q 6-12 h as per the severity and progression of the disease and when improvement is obtained less frequently. In the initial stages q 4-6 h may be appropriate and advisable. Supine cross-table lateral view may be done to visualize layering of free air under umbilical area if patient is too sick to move or put in a decubitus position (Figure 2). Radiography is discontinued when pneumatosis resolves and bowel gas pattern normalizes.

\subsection{Surgical management}

The only definite indication for surgery is intestinal perforation. Other relative indications, which are highly suggestive of bowel perforation or necrosis, are abdominal mass, fixed dilated bowel loop, positive paracentesis, and severe metabolic acidosis that is unresponsive to treatment (Figures 5 and 6) [46]. Signs that indicate peritonitis or bowel necrosis are unremitting clinical deterioration, worsening or unrelenting metabolic acidosis, and DIC or thrombocytopenia. Signs of ascites and intestinal obstruction may be present. Perforation can occur without evidence of free air on the radiograph as the timing of study may not coincide with the occurrence of perforation and the free air may get absorbed. Likewise, pneumatosis may not be always caught on serial X-rays. Under such conditions other signs and clinical judgment should be used to assess the severity and need for surgery. As clinical parameters may not be reliable to assess progression to surgical from medical NEC, abnormalities in biochemical markers, such as platelet count, CRP, WBC count, blood lactate, fecal calprotectin, and serum IL- 6 and IL- 8 may be used. Surgical procedures performed in cases of NEC are exploratory laparotomy with resection of the affected intestinal region or primary peritoneal drainage (PPD). PPD is preferred as the initial procedure in ELBW infants and is performed in the NICU at bedside with analgesia and local anesthesia. Laparotomy is done in an operating room under general anesthesia and may require a second surgical procedure for reanastomosis.

In primary peritoneal drainage abdomen is prepped with iodine solution, and local anesthesia is administered. Small transverse incision is made at McBurney's point and abdominal wall layers bluntly dissected to enter the peritoneal cavity. A rush of air and the presence of meconium are generally encountered. Cultures are obtained, and then peritoneal cavity is copiously irrigated with warm saline solution. Following this Penrose drain is gently threaded into the abdomen and secured. The drain site is observed over the subsequent days. When there is no intestinal or meconium drainage, the drain is backed out daily until removed. After the return of bowel function, a trial of feeding can be started, or the patency of the gastrointestinal tract may be determined with a contrast study. In laparotomy the procedure includes resection of the affected bowel segment and placement of a proximal enterostomy (usually an ileostomy) and distal mucous fistula. Primary reanastomosis, if required, usually is performed 8-12 weeks after the initial procedure, depending 


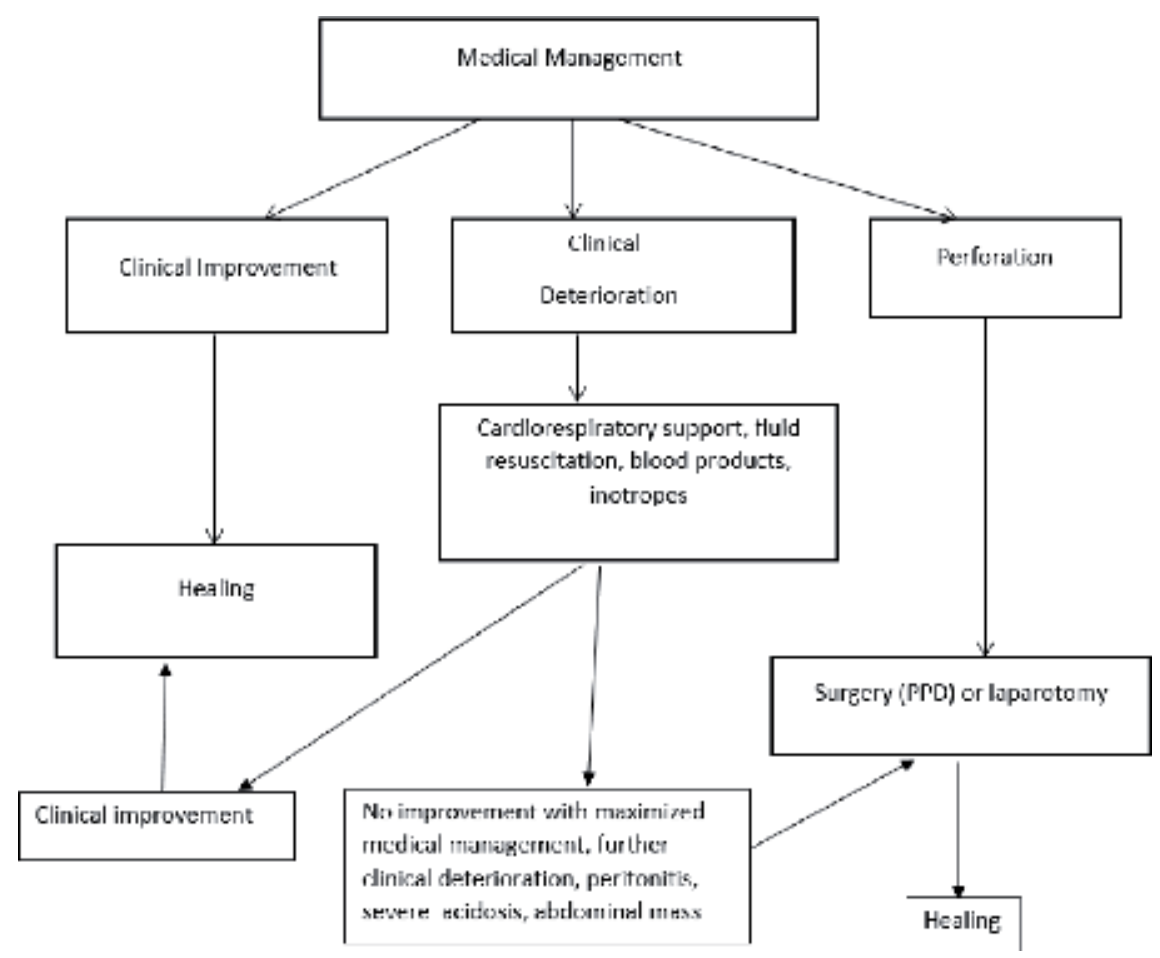

Figure 6.

Flow chart outlining management principles in NEC.

upon the infant's clinical condition. A contrast enema usually is performed before the reanastomosis to detect intestinal strictures. If NEC affects only a short segment of bowel, and the resection is limited, some surgeons perform a primary anastomosis. Complications associated with ileostomies are fluid and electrolyte abnormalities, delayed resumption of oral feedings, poor growth, and stenosis of the enterostomy site. An alternative approach is placement of an intestinal patch and peritoneal drain instead of resection and enterostomy. Preservation of ileocecal valve is a favorable prognostic sign. When a substantial length of bowel is affected, resection is restricted to segments of definite necrosis or perforation to avoid the risk of short bowel syndrome. If the potential viability of some segments is uncertain, one approach is to place peritoneal drains and plan a second operation in 2-3 days to reexamine the bowel and excise necrotic segments.

Recently, standard and fluorescein laparoscopy has been used in cases of NEC when there is no evidence of perforation, but clinical deterioration with maximum support continues, and a diagnosis for the presence and extent of bowel necrosis needs to be made in order to decide against, or in favor of, surgical exploration and its type [47]. Laparoscopy can also identify infants who do not need surgical intervention as it can visualize the viability and perfusion status of the bowel. According to a Cochrane review by Smith and Thyoka, which included eight reports and 44 patients, laparoscopy was able to diagnose NEC in $91 \%$ of the cases and exclude in $9 \%$ [48]. Moreover, additional surgical intervention was avoided in eight (18\%) infants. Among those who did not require surgery, NEC was excluded in four (9\%), while two $(5 \%)$ had no perforation or intestinal gangrene, and two $(5 \%)$ had NEC totalis which contraindicated surgery. Thirty-six infants out of 44 required surgery following laparoscopy, which included placement of a peritoneal drain (9) or a stoma (20) and intestinal resection and anastomosis (7). Perforation was detected in 25 out of $44(57 \%)$ infants and was missed in one case which subsequently 
required laparotomy. Six (14\%) infants died due to NEC totalis and two of ongoing and recurrent NEC following recovery from the acute episode of each. The authors concluded that laparoscopy is a useful procedure in the management of NEC, with one-fifth of patients not requiring further surgery. However, due to the lack of enough evidence about its utility and benefits, the procedure is generally not undertaken in the routine management of NEC.

\subsection{Comparison of laparotomy and PPD}

There are limited data about the superiority of one procedure over other. In a multicenter controlled study, 117 infants who were $<34$ weeks of gestation with perforated NEC were randomized to PPD or laparotomy. No differences were noted in mortality (34.5 versus 35.5\%), TPN dependence on postoperative day 90 (47.2 versus $40 \%$ ), and length of hospital stay (126 \pm 58 versus $116 \pm 56$ days) [49]. A subgroup analysis of cases with extensive pneumatosis intestinalis, GA less than 25 weeks, and serum $\mathrm{pH}$ less than 7.30 at presentation showed no significant advantage of one procedure over the other. In another randomized multicenter trial of 69 extremely low birth weight (ELBW) infants with NEC or SIP, no difference in the survival rates was noted between the two interventions, while 74\% treated with PPD subsequently required laparotomy [50]. A cohort study from the NICHD neonatal research group reported no difference in mortality rate, and 24\% treated with PPD required subsequent laparotomy. Blakely et al. reported that PPD is more likely to result in a composite outcome of death or neurodevelopmental impairment at 18-22 months postmenstrual age [51]. However in their study, PPD was performed in infants who were more premature (gestational age 24.7 versus 25.7 weeks), were more likely to be hypotensive, required higher respiratory support, and were more likely to have a preoperative diagnosis of SIP. Most of these studies are compromised by a lack pf power.

\subsection{Post-surgery intestinal failure and short bowel syndrome}

About $9 \%$ of NEC cases result in short bowel syndrome (SBS) with incidence inversely related to GA and almost $42 \%$ in intestinal failure and SBS $[52,53]$. Such infants suffer from significant malabsorption and are at risk for sepsis, cholestasis, and liver failure due to prolonged parenteral nutrition (PN). It is suggested that infants with residual small bowel length $\leq 10 \%$ of expected small bowel length may develop SBS. The cumulative probability of weaning from PN by 24 months is $96 \%$ in infants with $>50 \mathrm{~cm}$ of residual small bowel compared to $38 \%$ in those who have $<50 \mathrm{~cm}$ of residual small bowel. Those with $\geq 41 \mathrm{~cm}$ of residual bowel are significantly more likely to achieve enteral autonomy than those with less than $41 \mathrm{~cm}$ [53]. In general, those with post-resection length of remaining bowel less than $25 \%$ of the normal small bowel length have higher chances of developing SBS. The length of remaining bowel, however, is not the sole or best predictor of SBS. Infants at risk of SBS are as follows: parenteral antibiotics or mechanical ventilation on the day of onset of NEC, birth weight less than $750 \mathrm{~g}$, enteral nutrition before the diagnosis of NEC, percentage of bowel resected, and placement and duration of a diverting jejunostomy. The intestines continue to grow after resection and contribute to the process of attaining enteral autonomy.

\subsection{Surgical procedures for short bowel syndrome}

In a NICHD cohort, 95\% of cases of SBS were due to NEC with an overall incidence of $0.7 \%$ of infants $<1500 \mathrm{~g}$ at birth [52]. Approximately $42 \%$ of cases of NEC in infants $<1500 \mathrm{~g}$ of BW who undergo surgery develop intestinal failure and SBS, 
risk factors being lower birth weight, antibiotics use, positive pressure ventilation on the day of NEC, feeding, and lower post-resection length of remaining bowel [53]. Intestinal tailoring and lengthening procedure, also called "autologous intestinal reconstruction surgery" are performed in conditions where likelihood of weaning from $\mathrm{PN}$ is low despite rigorous intestinal rehabilitation measures. The criteria for surgery, tentative and not well supported by studies, include dilated small intestine, failure to attain intestinal autonomy, and absence of liver failure and GI dysmotility. The procedures aim at surgical lengthening of the bowel to increase the absorptive area and tapering or plicating the dilated bowel to improve motility. There is not enough literature on the subject to assess its clinical advantage. Bianchi procedure is a longitudinal intestinal lengthening and tailoring procedure (LILT) and may be successful in improving absorption and nutritional status but requires multiple anastomoses. Complications of this procedure include fistula formation, anastomotic stenosis, or leakage and sepsis [54]. The 6-year survival rate is $45 \%$, and survivors have residual bowel length greater than $40 \mathrm{~cm}$ and no liver disease. The Bianchi procedure is not recommended in neonates with liver disease or intestine length less than $50 \mathrm{~cm}$. The serial transverse enteroplasty procedure (STEP) is a substitute for LILT which increases the girth of bowel. It has a simpler technique that does not need bowel anastomoses, and the tapering can be performed on dilated bowel. STEP increases intestinal length, improves intestinal absorptive capacity, and may decrease the risk of D-lactic acidosis due to bacterial overgrowth. STEP is more favored over other techniques as the weaning from parenteral nutrition is faster and the need for later transplants lesser. There is no difference in early complications, growth rates, or survival between the two procedures, and nearly half of patients operated achieve enteral autonomy, with the median time to wean from parenteral nutrition (PN) being 21 months postoperatively. Patients whose bowel re-dilates after a lengthening procedure have worse overall outcomes. Complications of STEP include gastrointestinal bleeding, staple line leak, hematoma, abscess formation, stricture, pleural effusion, obstruction, a need for transplantation, and even death. In a recent publication, the rate of independence from $P N$ post-STEP was reported as $58 \%$ with parenteral calories decreasing from 71 to $36 \%$ within 1 month and to $12 \%$ after 1 year. The total increase in intestinal length achieved was about 49\% [54].

Small bowel transplantation (SBT) may be considered in patients who have progressive, severe, or irreversible intestinal failure with associated liver disease. Other considerations might be a lack of venous access, recurrent life-threatening central venous catheter-associated sepsis, complete mesenteric thrombosis, slow growing tumors of the hepatic hilum or root of mesentery, or extremely short residual bowel with no chance of achieving enteral autonomy in patients who prefer transplantation over lifelong PN dependence [54]. Small bowel transplantation has a 5-year graft survival rate of $48 \%$ with lifelong immunosuppressant medications. Types of transplantation include isolated intestine, isolated liver, combined liver and intestine, and multi-visceral. Complications are rejection, infection, graft-versus-host disease, and posttransplant lymphoproliferative disease. Overall, one year patient and intestine graft survival is $89 \%$, being $79 \%$ in intestine and $72 \%$ in liver-intestine graft recipients. Patient and intestine graft survival falls to $46 \%$ by 10 years. At 10 years the survival of intestine only recipients is $29 \%$, whereas, that of liver-intestine recipients $39-42 \%$. More recently, living donor intestinal transplantation has been done successfully in pediatric patients which eliminates waiting time.

\subsection{Intestinal rehabilitation}

This requires a multidisciplinary approach. The focus is on optimizing enteral feeding and weaning from PN with judicious use of pharmacotherapy and surgical 
interventions. The approach is associated with improved survival and achievement of independence from $\mathrm{PN}$.

\section{Complications}

The acute complications of NEC are sepsis, meningitis, peritonitis, intraabdominal abscess formation, DIC, thrombocytopenia, hypotension, shock, respiratory failure, metabolic or combined acidosis, hyponatremia, hyperglycemia, or less often hypoglycemia. Late complications are stricture formation, short bowel syndrome, and intestinal failure [55]. Rarely enterocele, enterocolic fistula, and intraabdominal abscess formation may be encountered. About 24\% (95\% CI 17-31\%) of infants treated medically or surgically develop strictures in bowel which is unrelated to the severity of NEC or gestational age. The commonest location is in the colon, followed by the ileum and jejunum. Multiple sites strictures are seen. It can appear within 2 to 3 months of the acute episode and as late as 20 months. Stricture may lead to local bacterial overgrowth resulting in repeated infections, bloody stools, failure to thrive, and symptoms of bowel obstruction. Strictures are more common following enterostomy; therefore contrast enemas should be performed 4-6 weeks after the occurrence of NEC and prior to surgical closure of enterostomy with reanastomosis or if and when feeding intolerance develops. Recurrent NEC may occur in $8 \%$ and adhesion ileus in 6\% cases of NEC. Overall intestinal failure happens in $13 \%$ of all cases of NEC, inclusive of medically and surgically treated infants.

\section{Long-term outcomes}

Approximately half of all cases of NEC display no long-term sequelae. This includes cases of stage I NEC from Bell's criteria. NEC is associated with significant impairment of growth and neurodevelopment [56-58]. Ten to thirteen percent of patients suffer from late gastrointestinal morbidity if resection is performed. Majority of infants who have had no extensive intestinal resection have normal gastrointestinal function at 1-10 years of age.

\subsection{Growth and neurodevelopment}

In a large multicenter study from the National Institute of Child Health and Human Development (NICHD) Neonatal Research Network, extremely low birth weight $($ ELBW) infants (BW $<1000 \mathrm{~g}$ ) who required surgical care were more likely to have significant growth delay and poorer developmental outcome at 18-22 months compared with infants without NEC [56]. ELBW treated medically or surgically suffer from significant growth failure until 22 months, and in a lesser percentage beyond that age [57], while those who were medically treated do not differ in growth or developmental testing compared with those without NEC [58]. Infants with NEC are at increased risk for cerebral palsy and cognitive and severe visual impairment. At 7 years of age, survivors with NEC compared to controls demonstrate a higher rate of neurologic functional impairment.

\subsection{Mortality}

Overall survival in cases with NEC is $70-80 \%$, being $>95 \%$ in medical and $70-75 \%$ in surgical NEC [59]. Lower BW and GA and surgical intervention, such as laparotomy and peritoneal drainage, are independent predictors for mortality. 
Other risk factors associated with death from NEC are mechanical ventilation, treatment with vasopressor agents, surgical intervention, and black ethnicity as per a retrospective multicenter review of data [60]. Two thirds of NEC deaths occur within 7 days of diagnosis, with a median time of death being 1 day from the day of onset. Infants who die within 7 days of diagnosis have higher BW, and more often they are on vasopressors and high-frequency ventilation at the time of diagnosis. Risk factors for fulminant NEC, defined as death within $48 \mathrm{~h}$ of onset, are presence of portal venous air, increase in feeding volume by $>20 \mathrm{~mL} / \mathrm{kg}$ per day, HCT $<22 \%$, $\mathrm{I} / \mathrm{T}$ ratio $>0.5$, and total lymphocyte count $<4000 / \mu \mathrm{L}$ as demonstrated in a multicenter study [61].

\subsection{Financial burden of NEC}

The average total treatment cost of one case of NEC is US $\$ 500,000[62,63]$. Total annual estimated cost of care of NEC in the United States is between $\$ 500$ million and $\$ 1$ billion. Infants with NEC are hospitalized for 60 days longer than unaffected preterm infants if surgery is performed and $>20$ days longer if surgery is not required. Bowel resection - one of the most severe complications of NEC-is the major cause of short bowel syndrome in pediatric patients, making $95 \%$ of all such cases. The total mean cost of care over a 5-year period for a child with the short bowel syndrome has been estimated to be nearly $\$ 1.5$ million.

\section{Prevention}

Prevention is the primary strategy in this devastating disease with undetermined etiology. Breast milk feeding, prolonging gestation to avoid prematurity, antenatal steroid, and the use of probiotics/prebiotics are established prevention strategies in NEC [16]. Nonaggressive feeding is evidenced to be efficacious. The rate of advancement of feeding under $20 \mathrm{ml} / \mathrm{kg} /$ day is considered to be safe. Newer strategies, such as use of toll-like receptor agonist, glutamine, n-3 fatty acids, anti-cytokines, and growth factors are proposed preventive interventions, but most of these either lack evidence or have questionable safety. Compound CpG-DNA inhibit TLR4 signaling, thereby dramatically reducing the severity of NEC in mice. Clinically, the following measures are suggested to be practiced in order to reduce the risk of NEC: human milk (both mother's and donor's); standardized feeding guidelines, including early initiation with trophic feeds; the use of probiotics; antibiotic stewardship; optimization of enteral nutrition and growth; elimination of $\mathrm{H} 2$ blockers and acid pump suppressors; elimination of cow's milk products; transfusion protocols; and transfusion outcome monitoring. Avoidance of hyperosmolar agents, treatment of polycythemia, and delayed cord clamping are other interventions that are suggested to be followed. Prophylactic probiotics, although not yet universally applied due to uncertainties about its dose and duration of therapy, have been documented to reduce the incidence of NEC, especially that of severe cases (RR $0.7595 \% \mathrm{CI}-0.57$ to 0.92 ) in infants $<1500 \mathrm{~g}$ in multiple studies [64]. There are concerns about bacteremia and some aspects of quality control which restrict its use.

\section{Future directions}

NEC remains a major unsolved medical challenge for which no specific therapy exists. Recent research is concentrated on the role of TLR4 signaling within the intestinal epithelium and intestinal stem cells and modulation of the genetics and 
intestinal microbiome. Fecal microbiota transplantation (FMT) has been shown to reverse the severity of experimental necrotizing enterocolitis (NEC) via oxidative stress modulation [65]. FMT decreases the extent of TLR4-mediated pro-inflammatory signaling through TLR9 in the intestinal mucosa tissue. FMT also suppresses intestinal apoptosis and bacterial translocation across the intestinal barrier, which is accompanied by decreased inflammatory cytokine levels, altered bacterial microbiota, and regulated lymphocyte proportions. Research is needed to determine if the use of biomarkers along with specific clinical-biochemical indicators could lead to earlier intervention with modalities, such as peritoneal drainage or laparotomy that might decrease the severity of the disease process, thereby improving the long-term neurodevelopmental and growth outcomes. Improved care of short bowel syndrome with new surgical and medical approaches are additional subject for investigation. Tissue engineering techniques and techniques involving intestinal stem cells may represent unique, novel strategies for intestinal failure after severe NEC in the future.

\section{Author details}

Rita Prasad Verma* and Archana Kota

Department of Pediatrics, Nassau University Medical Center, East Meadow, NY, USA

*Address all correspondence to: rverma2@numc.edu

IntechOpen

(C) 2019 The Author(s). Licensee IntechOpen. This chapter is distributed under the terms of the Creative Commons Attribution License (http://creativecommons.org/licenses/ by/3.0), which permits unrestricted use, distribution, and reproduction in any medium, provided the original work is properly cited. (cc) BY 


\section{References}

[1] Yee WH, Soraisham AS, Shah VS, et al. Incidence and timing of presentation of necrotizing enterocolitis in preterm infants. Pediatrics. 2012;129(2):e298-e304. DOI: $10.1542 /$ peds.2011-2022

[2] Stoll BJ, Hansen NI, Bell EF, et al. Trends in care practices, morbidity, and mortality of extremely preterm neonates, 1993-2012. JAMA.

2015;314:1039

[3] Niño DF, Sodhi CP, Hackam DJ. Necrotizing enterocolitis: New insights into pathogenesis and mechanisms. Nature Reviews. Gastroenterology \& Hepatology. 2016;13:590

[4] Sharma R, Tepas JJ 3rd, Hudak ML, et al. Neonatal gut barrier and multiple organ failure: Role of endotoxin and proinflammatory cytokines in sepsis and necrotizing enterocolitis. Journal of Pediatric Surgery. 2007;42(3):454-461

[5] Holman RC, Stoll BJ, Curns AT, et al. Necrotising enterocolitis hospitalisations among neonates in the United States. Paediatric and Perinatal Epidemiology. 2006;20:498

[6] Battersby B, Santhalingam T, Costeloe K, Modi N. Incidence of neonatal necrotising enterocolitis in high-income countries: A systematic review. Archives of Disease in Childhood: Fetal \& Neonatal. 2018;103:F182-F189. DOI: 10.1136/ archdischild-2017-313880

[7] Rees CM, Eaton S, Pierro A. National prospective surveillance study of necrotizing enterocolitis in neonatal intensive care units. Journal of Pediatric Surgery. 2010;45:1391-1397

[8] Henry MC, Moss LR. Surgical therapy for necrotizing enterocolitis: Bringing evidence to the bedside. Seminars in Pediatric Surgery. 2005;14:181-190
[9] Guillet R, Stoll BJ, Cotten CM, et al. Association of H2-blocker therapy and higher incidence of necrotizing enterocolitis in very low birth weight infants. Pediatrics. 2006;117:e137-e142

[10] Stoll BJ, Hansen NI, Bell EF, et al. Neonatal outcomes of extremely preterm infants from the NICHD Neonatal Research Network. Pediatrics. 2010;126:443-456

[11] Lin HC, Wu SF, Underwood M. Necrotizing enterocolitis. The New England Journal of Medicine. 2011;364:1878-1879

[12] Fanaroff AA, Stoll BJ, Wright LL, Carlo WA, Ehrenkranz RA, Stark AR, et al. Trends in neonatal morbidity and mortality for very low birthweight infants. American Journal of Obstetrics and Gynecology. 2007;196(2):147

[13] Hull MA, Fisher JG, Gutierrez IM, Jones BA, Kang KH, Kenny M, et al. Mortality and management of surgical necrotizing enterocolitis in very low birth weight neonates: A prospective cohort study. Journal of the American College of Surgeons. 2014;18(6):1148-1155

[14] Reese CM, Pierro A, Eaton S. Neurodevelopmental outcomes of neonates with medically and surgically treated necrotizing enterocolitis. Archives of Disease in Childhood: Fetal and Neonatal Edition. 2007;92(3):F193-F198

[15] Robinson JR, Rellinger EJ, Hatch LD, Weitkamp J, Speck E, Danko M, et al. Surgical necrotizing enterocolitis. Seminars in Perinatology. 2017;41(1):70-79

[16] Patel AL, Panagos PG, Silvestri JM. Reducing incidence of necrotizing enterocolitis. Clinics in Perinatology. 2017;44:683-700 
[17] Uauy RD, Fanaroff AA, Korones $\mathrm{SB}$, et al. Necrotizing enterocolitis in very low birth weight infants: Biodemographic and clinical correlates. National Institute of Child Health and Human Development Neonatal Research Network. Journal of Pediatrics. 1991;119:630

[18] Berseth CL. Feeding strategies and necrotizing enterocolitis. Current Opinion in Pediatrics. 2005;17:170

[19] Gephart SM, Spitzer AR, Effken JA, Dodd E, Halpern M, McGrath JM. Discrimination of GutCheck (NEC): A clinical risk index for necrotizing enterocolitis. Journal of Perinatology. 2014;34(6):468-475

[20] Lambert DK, Christensen RD, Henry E, et al. Necrotizing enterocolitis in term neonates: Data from a multihospital health-care system. Journal of Perinatology. 2007;27:437-443

[21] Neu J. The 'myth' of asphyxia and hypoxia-ischemia as primary causes of necrotizing enterocolitis. Biology of the Neonate. 2005;87:97-98

[22] Liu Z, Li N, Neu J. Tight junctions, leaky intestines, and pediatric diseases. Acta Paediatrica. 2005;94(4):386-393

[23] Neu J, Walker WA. Necrotizing enterocolitis. The New England Journal of Medicine. 2011;364(3):255-264

[24] Ballance WA, Dahms BB, Shenker N, Kliegman RM. Pathology of neonatal necrotizing enterocolitis: A ten year experience. The Journal of Pediatrics. 1990;117:S6-S13

[25] Frost BL, Modi BP, Jaksic T, Caplan MS. New medical and surgical insights into neonatal necrotizing enterocolitis: A review. JAMA Pediatrics. 2017;171(1):83-88

[26] Treszl A, Tulassay T, Vasarhelyi B. Genetic basis for necrotizing
enterocolitis-Risk factors and their relations to genetic polymorphisms. Frontiers in Bioscience. 2006;11(2006):570-580

[27] Sharma R, Young C, Neu J. Molecular modulation of intestinal epithelial barrier: Contribution of microbiota. Journal of Biomedicine \& Biotechnology. 2010:305879. DOI: 10.1155/2010/305879. [Epub 2010 Jan 31]

[28] Lin PW, Nasr TR, Stoll BJ. Necrotizing enterocolitis: Recent scientific advances in pathophysiology and prevention. Seminars in Perinatology. 2008;32(2):70-82

[29] Hunter CJ, Upperman JS, Ford HR, Camerini V. Understanding the susceptibility of the premature infant to necrotizing enterocolitis (NEC). Pediatric Research. 2008;63:117-123

[30] Denning TL, Bhatia AM, Kane AF, et al. Pathogenesis of NEC: Role of the innate and adaptive immune response. Seminars in Perinatology. 2017;41:15-28

[31] Tanner SM, Berryhill TF, Eelenburg JL, et al. Pathogenesis of NEC:

Modelling the innate immune response. American Journal of Pathology. 2015;185(1):4-16

[32] Maheshwari A, Schelonka RL, Dimmitt RA, et al. Cytokines associated with necrotizing enterocolitis in extremely-low-birth-weight infants. Pediatric Research. 2014;76(1):100-108

[33] Sodhi CP et al. Toll-like receptor-4 inhibits enterocyte proliferation via impaired $\beta$-catenin signaling in necrotizing enterocolitis. Gastroenterology. 2010;138:185-196

[34] Lu P, Sodhi CP, Hackam DJ. Tolllike receptor regulation of intestinal development and inflammation in the pathogenesis of necrotizing enterocolitis. Pathophysiology. 2014;21:81-93 
[35] Yazji I et al. Endothelial TLR4 activation impairs intestinal microcirculatory perfusion in necrotizing enterocolitis via eNOSNO-nitrite signaling. Proceedings of the National Academy of Sciences of the United States of America. 2013;110:9451-9456

[36] Ostlie DJ, Spilde TL, St Peter SD, et al. Necrotizing enterocolitis in full-term infants. Journal of Pediatric Surgery. 2003;38:1039-1042

[37] Walsh MC, Kliegman RM. Necrotizing enterocolitis: Treatment based on staging criteria. Pediatric Clinics of North America. 1986;33:179-201

[38] Fortune PM, Wagstaff M, Petros AJ. Cerebro-splanchnic oxygenation ratio (CSOR) using near infrared spectroscopy may be able to predict splanchnic ischaemia in neonates. Intensive Care Medicine. 2001;27:1401-1407

[39] Munaco AJ, Veenstra MA, Brownie E, Danielson LA, Nagappala KB, Klein MD. Timing of optimal surgical intervention for neonates with necrotizing enterocolitis. The American Surgeon. 2015;81:438-443

[40] Zani A, Eaton S, Puri P, et al. International survey on the management of necrotizing enterocolitis. European Journal of Pediatric Surgery. 2015;25:27-33

[41] Aydemir O, Aydemir C, Sarikabadayi YU, et al. Fecal calprotectin levels are increased in infants with necrotizing enterocolitis. The Journal of Maternal-Fetal \& Neonatal Medicine. 2012;25:2237-2241

[42] Benkoe T, Reck C, Gleiss A, et al. Interleukin 8 correlates with intestinal involvement in surgically treated infants with necrotizing enterocolitis. Journal of Pediatric Surgery. 2012;47:1548-1554
[43] Benkoe T, Reck C, Pones M, et al. Interleukin-8 predicts 60 -day mortality in premature infants with necrotizing enterocolitis. Journal of Pediatric Surgery. 2014;49:385-389

[44] Gaudin A, Farnoux C, Bonnard A, et al. Necrotizing enterocolitis (NEC) and the risk of intestinal stricture: The value of C-reactive protein. PLoS One. 2013;8:e76858

[45] Guthmann F, Borchers T, Wolfrum C, Wustrack T, Bartholomaus S, Spener F. Plasma concentration of intestinal- and liver-FABP in neonates suffering from necrotizing enterocolitis and in healthy preterm neonates. Molecular and Cellular Biochemistry. 2002;239:227-234

[46] Tepas JJ 3rd, Sharma R, Leaphart CL, Celso BG, Pieper P, Esquivia-Lee V. Timing of surgical intervention in necrotizing enterocolitis can be determined by trajectory of metabolic derangement. Journal of Pediatric Surgery. 2010;45:310-313

[47] Lacher M, Kuebler FK, Dingemann J, Ure BM. Minimal invasive surgery in the newborn: Current status and evidence. Seminars in Pediatric Surgery. 2014;23:249-256

[48] Smith J, Thyoka M. What role does laparoscopy play in the diagnosis and immediate treatment of infants with necrotizing enterocolitis? Journal of Laparoendoscopic \& Advanced Surgical Techniques. 2013;4:397-401

[49] Rao SC, Basani L, Simmer K, Samnakay N, Deshpande G. Peritoneal drainage versus laparotomy as initial surgical treatment for perforated necrotizing enterocolitis or spontaneous intestinal perforation in preterm low birth weight infants. Cochrane Database of Systematic Reviews. 2011;6:CD006182. DOI: 10.1002/14651858.CD006182.pub2 
[50] Rees CM, Eaton S, Kiely EM, et al. Peritoneal drainage or laparotomy for neonatal bowel perforation? A randomized controlled trial. Annals of Surgery. 2008;248:444-491

[51] Blakely ML, Tyson JE, Lally KP, et al. Laparotomy versus peritoneal drainage for necrotizing enterocolitis or isolated intestinal perforation in extremely low birth weight infants: Outcomes through 18 months adjusted age. Pediatrics. 2006;117:e680

[52] Cole CR, Hansen NI, Higgins RD, Ziegler TR, Stoll BJ. Very low birth weight preterm infants with surgical short bowel syndrome: Incidence, morbidity and mortality, and growth outcomes at 18 to 22 months. Pediatrics. 2008;122:e573-e582

[53] Duro D, Kalish LA, Johnston P, et al. Risk factors for intestinal failure in infants with necrotizing enterocolitis: A Glaser Pediatric Research Network study. Journal of Pediatrics. 2010;157(2):203-208.e1

[54] Amin SC, Pappas C, Iyengar H, Maheshwari A. Short bowel syndrome in the NICU. Clinics in Perinatology. 2013;40(1):53-68

[55] Schwartz MZ, Richardson CJ, Hayden CK, et al. Intestinal stenosis following successful medical management of necrotizing enterocolitis. Journal of Pediatric Surgery. 1980;15:890

[56] Hintz SR, Kendrick DE, Stoll BJ, et al. Neurodevelopmental and growth outcomes of extremely low birth weight infants after necrotizing enterocolitis. Pediatrics. 2005;115:696

[57] Hong CR, Fullerton BS, Mercier $\mathrm{CE}$, et al. Growth morbidity in extremely low birth weight survivors of necrotizing enterocolitis at discharge and two-year follow-up. Journal of Pediatric Surgery. 2018;53:1197-1202
[58] Schulzke SM, Deshpande GC, Patole SK. Neurodevelopmental outcomes of very low-birth-weight infants with necrotizing enterocolitis: A systematic review of observational studies.

Archives of Pediatrics \& Adolescent Medicine. 2007;161:583

[59] Fitzgibbons SC, Ching Y, Yu $\mathrm{D}$, et al. Mortality of necrotizing enterocolitis expressed by birth weight categories. Journal of Pediatric Surgery. 2009;44:1072-1075

[60] Clark RH, Gordon P, Walker WM, Laughon M, Smith PB, Spitzer AR. Characteristics of patients who die of necrotizing enterocolitis. Journal of Perinatology. 2011;32(3):199-204

[61] Lambert DK, Christensen RD, Baer VL, et al. Fulminant necrotizing enterocolitis in a multihospital healthcare system. Journal of Perinatology. 2012;32:194

[62] Stey A et al. Outcomes and costs of surgical treatments of necrotizing enterocolitis. Pediatrics. 2015;135:e1190-e1197

[63] Bisquera JA, Cooper TR, Berseth CL. Impact of necrotizing enterocolitis on length of stay and hospital charges in very low birth weight infants. Pediatrics. 2002;109:423-428

[64] Thomas JP, Raine T, Reddy S, Belteki G. Probiotics for the prevention of necrotising enterocolitis in very lowbirth-weight infants: a meta-analysis and systematic review. Acta Paediatr 2017;106:1729-1741

[65] Li X, Li X, Shang Q, Gao Z, Hao F, Guo H, et al. Fecal microbiota transplantation (FMT) could reverse the severity of experimental necrotizing enterocolitis (NEC) via oxidative stress modulation. Free Radical Biology \& Medicine. 2017;108:32-43 


\title{
Oesophageal Atresia: Drowning a Child in His/Her Own Saliva
}

\author{
Samuel Osei-Nketiah and William Appeadu-Mensah
}

\begin{abstract}
Oesophageal atresia (OA) is a congenital anomaly characterised by absence or loss of a segment of the oesophagus. This commonly affects the thoracic portion of the oesophagus, leaving upper and lower oesophageal segments. Loss of the oesophageal luminal continuity leads to impaired in utero swallowing of amniotic fluid as well as postnatal swallowing of saliva and food. Besides the loss of oesophageal continuity, most of the patients tend to have a connection between the trachea and the lower oesophageal segment and a few between the trachea and the upper oesophageal segment, a condition called tracheo-oesophageal fistula (TOF). In view of these, the main principles guiding the definitive surgical management of OA are (1) to disconnect any TOF and (2) to establish a conduit for swallowing, preferably using the native oesophageal segments. This chapter seeks to discuss OA by focusing on the embryology, anatomy and physiology of the oesophagus, stressing on the embryological basis of OA. Other areas to cover include aetiology, pathogenesis, epidemiology, pathologic classification, associated anomalies, pathophysiology, clinical presentation and diagnosis. Further discussion will focus on prognostic classification of patients, management and post-operative complications.
\end{abstract}

Keywords: oesophageal atresia, pathology, associated anomalies, pathophysiology, clinical presentation, diagnosis, pre-operative management, surgical management, outcome, prognosis

\section{Introduction}

Oesophageal atresia (OA), a congenital anomaly characterised by absence or loss of a segment of the oesophagus, commonly affects the thoracic portion of the oesophagus, leaving upper and lower oesophageal segments. Loss of the oesophageal luminal continuity leads to impaired in utero swallowing of amniotic fluid as well as postnatal swallowing of saliva and food. Besides the loss of oesophageal continuity, most of the patients tend to have a connection between the trachea and the lower oesophageal segment and a few between the trachea and the upper oesophageal segment, a condition called tracheo-oesophageal fistula (TOF). In view of these, the main principles guiding the definitive surgical management of oesophageal atresia are (1) to disconnect any tracheo-oesophageal fistula and (2) to establish a conduit for swallowing, preferably using the native oesophageal segments.

During the early years, the surgical management of oesophageal atresia was associated with lots of challenges and high mortality [1-4]. Over the past two to three decades, however, the surgical outcome has improved significantly in most centres in the developed countries. This improvement is attributed to advances 


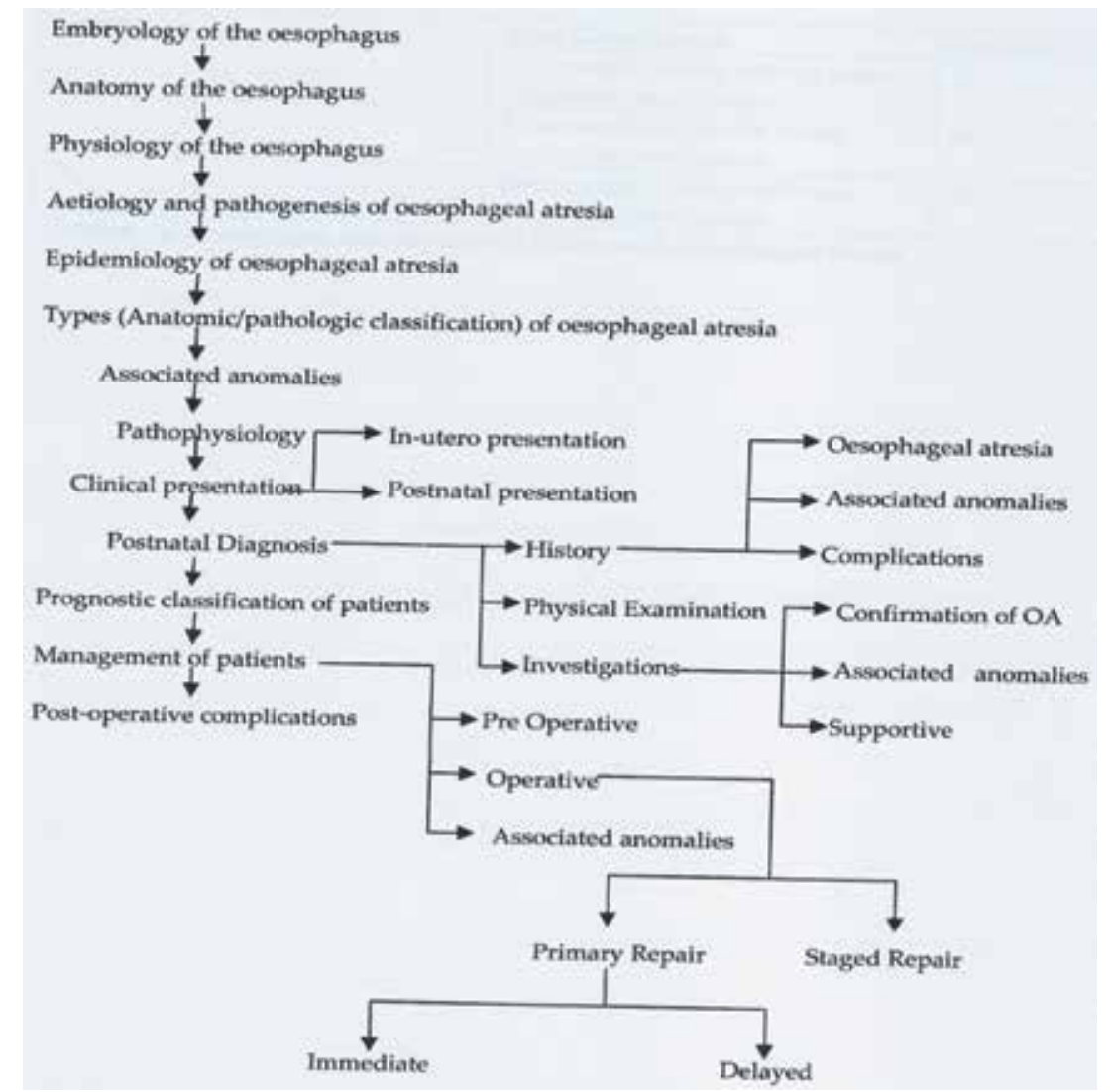

Figure 1.

Flow chart showing various topics to be discussed under oesophageal atresia.

in neonatal anaesthesia, well-established neonatal intensive care units (NICU), availability of total parental nutrition (TPN) and refined surgical skills [1, 5-8]. Conversely, the surgical outcome of oesophageal atresia in developing countries still remains very poor due to lack of the aforementioned facilities, in addition to late presentation [9-11].

This chapter seeks to discuss OA by focusing on the topics shown in Figure 1.

\section{Embryology of the oesophagus}

The oesophagus develops from the primitive foregut as a continuation of the pharynx. It is said to be present by the fifth week of gestation, and it attains its final foetal length $(8-10 \mathrm{~cm})$ during the 7 th week of gestation [12]. Thus, the length of the oesophagus at birth is $8-10 \mathrm{~cm}$, and this doubles during the first few years of life [12].

The normal embryology of the foregut, as found in most reports and textbooks of embryology, is divided into five developmental steps [13]:

1. During the first step, the endoderm (epithelium) of the primitive foregut differentiates into a ventral area called the lung field and a dorsal area called oesophageal area. The epithelium of the ventral area (lung field) of the primitive foregut consists of 3-4 cell layers, while that of the dorsal area (oesophageal area) has only one cell layer. This phase occurs when the embryo is about 22-23 days old. 
2. Lung (tracheal) bud develops at the caudal end of the lung field.

3. During the third step, beginning caudally at the area of the lung bud, the lateral walls of the foregut start to approximate, developing longitudinal ridges inside the lumen of the foregut. This clearly separates the ventral lung field and the dorsal oesophageal area.

4. Epithelial tracheo-oesophageal septum develops during the fourth step; and it is assumed that this process also starts caudally and ends cranially close to the laryngeal primordium. This process is described by most investigators in four steps: (i) the epithelium of the longitudinal ridges starts to proliferate; (ii) the ridges, therefore, fuse in the midline of the primitive foregut and form an epithelial septum; (iii) cell death takes place in the central areas of the septum, noticeable by the appearance of nuclei pyknosis and (iv) as a result, mesenchymal tissue then expands into the area between the trachea and the oesophagus.

5. Separation of the respiratory tract from the oesophagus becomes definitive between the sixth and the seventh weeks of gestation through the formation of a mesenchymal septum called tracheo-oesophageal septum.

It should be noted that most steps in this schematic description of the foregut embryology lack clear evidence [13].

Other developmental features of the oesophagus include [12]:

Mesenchymal circular coat (muscle) develops early in the sixth week of gestation. The longitudinal muscle forms between the ninth and twelfth weeks of gestation, and the muscularis mucosa develops at approximately the fourth month of gestation [12].

Blood vessels enter the oesophageal wall during the seventh month of gestation and lymph capillaries between the third and fourth months of life [12]. The most important embryologic structure for blood supply to the oesophagus is the fourth branchial arch. The arch produces the subclavian artery and its branches, including the inferior thyroid artery which supplies the cervical oesophagus. The fourth branchial arch also produces the aorta, from which vessels spring to supply the thoracic oesophagus.

The formation of the oesophageal epithelium is peculiar; it "changes face" four times. The epithelium is stratified columnar at the start of embryonic life, becoming cuboidal later. In foetal life, it is ciliated columnar, and, finally, it becomes stratified squamous soon after birth. Innervation of the oesophageal wall is received from sympathetic nerve fibres from the thoracic trunk and celiac plexus and parasympathetic innervation from the vagus nerve.

The oesophageal wall is formed from endoderm and mesoderm (Figure 2). The endoderm produces the oesophageal epithelium and glands, whereas the mesoderm produces the connective tissue, muscular coat, and angioblasts. Splanchnic mesenchyme surrounds the oesophagus and trachea. The splanchnic mesenchyme forms the smooth muscle of the lower oesophagus.

The causal branchial arches (4 and 6) are responsible for the formation of the striated musculature of the upper oesophagus and pharynx. They are innervated by the vagus nerve (nerve to the fourth arch) and the recurrent laryngeal nerve branch of the vagus nerve (nerve to the sixth arch) [12].

The oesophageal lumen is almost filled with vacuolated cells from proliferation of oesophageal epithelium during the seven-eighth weeks of gestation. The filling is never complete, and hence the so-called solid stage does not exist. At 10 weeks' gestation, the lumen of the oesophagus is restored as the vacuolated cells disappear. 


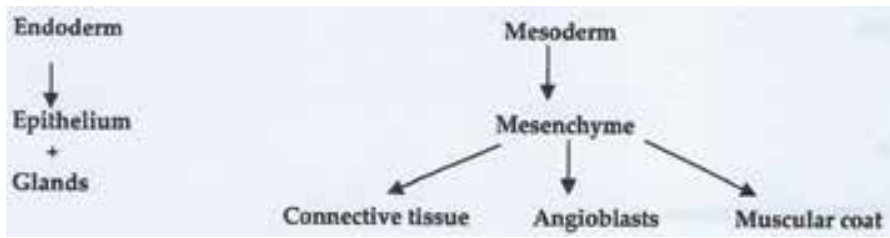

Figure 2.

Embryologic duo responsible for genesis of the oesophagus.

\subsection{Anatomy of the oesophagus}

The oesophagus is a muscular tube connecting the pharynx to the stomach. At birth, the length of the oesophagus is about $8-10 \mathrm{~cm}$, and this doubles in the first few years of life. The length of the oesophagus in the adult is about $25 \mathrm{~cm}$. It extends from the lower border of the cricoid cartilage (at the level of the C6 vertebra) to the cardiac orifice of the stomach at the level of T11 vertebra. The upper limit in the newborn is found at the level of the fourth or fifth cervical vertebra and ends higher at the level of the T9 vertebra $[14,15]$.

The oesophageal wall is composed of mucosa, submucosa, muscularis propria and adventitia, lacking a distinct serosa. The mucosa is the strongest layer of the oesophageal wall. Hence, meticulous approximation of the oesophageal mucosa is essential for a technically sound anastomosis.

The oesophagus is divided into three segments-cervical, thoracic and abdominal segments. The cervical portion is somewhat curved, with its convex side to the left, thereby projecting to the left of the trachea. Incisions for approaching the cervical oesophagus are commonly made on this side. Anteriorly, the cervical oesophagus is covered by the trachea.

The arterial blood supply to the oesophagus is generally considered with regard to the cervical, thoracic and abdominal segments of the oesophagus. The arterial blood supply to the pharyngo-oesophageal junction and the cervical oesophagus is derived from branches of the inferior thyroid artery. In addition, the pharyngo-oesophageal junctional area of the oesophagus is supplied by small arterial branches of the subclavian (artery of Luschka), common carotid, vertebral, superior thyroid and costocervical trunk vessels $[12,16]$. The thoracic oesophagus is supplied from oesophageal branches of the aorta, the bronchial arteries and the right intercostal arteries. Accessory oesophageal branches are also present directly from the internal mammary, common carotid and superior intercostal arteries $[12,17]$. The left gastric artery provides oesophageal blood supply to the abdominal segment of the oesophagus in most individuals. Rarely, oesophageal arteries will arise from an accessory left hepatic artery. In less than one-half of individuals, the oesophagus receives arterial blood via the left inferior phrenic artery and rarely from the right inferior phrenic artery [16]. A welldeveloped subepithelial network of capillaries is present in the oesophageal mucosa and submucosa $[18,19]$. The excellent submucosa plexus of the proximal oesophagus allows for extensive mobilization without compromise to the blood supply, whereas caution should be taken distally because of the segmental lower oesophageal blood supply.

Venous drainage from the oesophagus includes intrinsic and extrinsic vessels. The intrinsic system includes subepithelial and submucosal veins that join gastric veins and perforating veins that join with the extrinsic system of veins. The extrinsic veins include larger longitudinal vessels that run on the outer surface of the oesophagus and are close to the vagus nerves. These vessels connect the left gastric vein to the azygous or hemiazygous veins either directly or indirectly via the posterior bronchial veins. Extrinsic veins drain into the inferior thyroid, vertebral and deep cervical veins in the cervical region. Oesophageal veins at the level of the 
cardia join the phrenic and abdominal oesophageal veins to drain primarily into the left gastric vein, as well as the gastroepiploic and splenic veins [20]. This may be a point of importance when dealing with a patient with portal hypertension.

The oesophageal lymphatics form plexuses in the mucosa (lamina propria), submucosa, muscularis, and adventitia with interconnectivity [12]. Collecting trunks originate in the submucosa and empty into the nearest lymph nodes.

In the oesophageal wall are two plexuses of nerves for intrinsic nerve supply: (i) Meissner's plexus in the submucosa and (ii) Auerbach's plexus in the connective tissue between the circular and longitudinal muscularis externa [12]. These plexuses form networks of multipolar ganglion cells, the processes of which are in contact with one another and receive axons from the vagus. The oesophagus receives extrinsic nerve supply from three sources: the (a) cerebrospinal, (b) sympathetic and (c) parasympathetic (vagal) nervous systems [12].

The cricopharyngeal (CP) muscle, which is located at the pharyngo-oesophageal junction, attaches to the cricoid cartilage and forms a C-shaped muscular band. It is innervated by the pharyngeal plexus of the vagus nerve and the recurrent laryngeal nerve [21]. The main function of the CP muscle is to control luminal flow between the pharynx and oesophagus. The CP sphincter muscle is tonically contracted at rest and relaxes during swallowing. The major component of the upper oesophageal sphincter (UES) is the CP muscle, although the inferior pharyngeal constrictor and striated muscles of the proximal oesophagus also contribute [22].

The function of the lower oesophageal sphincter is abolished by total truncal vagotomy.

\subsection{Physiology of the oesophagus}

The main function of the oesophagus is for swallowing, and this is achieved through peristalsis. Functionally, the oesophagus is divided into three areas: (i) the upper oesophageal sphincter (UOS), (ii) the oesophageal body and (iii) the lower oesophageal sphincter (LOS). The coordinated activity of these three parts is essential to ensure propulsion of bolus from the pharynx to the stomach. The UOS plays a key role in controlling regurgitation of oesophageal content into the pharynx and the airways, while the LOS prevents reflux of gastric content into the oesophagus.

\subsection{Aetiology and pathogenesis of oesophageal atresia}

Various theories were developed in the past to explain the embryology of foregut anomalies. These theories are grouped into four [13]: (i) oesophageal occlusion theory, (ii) theories of spontaneous deviation of the tracheo-oesophageal septum, (iii) mechanical theories and (iv) not otherwise specified (NOS) theories.

Tandler postulated the theory of foregut occlusion in 1902 as a physiological occlusion during duodenal development [13]. Such physiological occlusion is also postulated to occur during oesophageal development; and that failure of recanalisation leads to oesophageal atresia [13]. Tracheo-oesophageal septal deviation is found to be another theory that explains the development of OA [13]. Various mechanisms have been used to explain the mechanical theory [13]. These include ventral pressure on the developing oesophagus by a very big anlage of the heart and aberrant vessels. The NOS theories include the development of a very large tracheal field that uses too much tissue to form the trachea, resulting in a shortage of dorsal tissue. Abnormal septation, combined with a disturbance in the organ inducing field, is believed to account for OA with TOF. Isolated TOF is speculated to result from a loss of epithelial proliferation or through an excessive necrosis in the area of the epithelial tracheo-oesophageal septum [13]. 
Aetiologically, various genetic defects have been found to be associated with oesophageal atresia. Important genes related to the pathogenesis of OA, and mostly involved in developmental pathways, include vitamin A effectors, retinoic acid receptors a and $b$ (Rara and Rarb), sonic hedgehog pathway effectors (Shh, Gli2, Gli3 and Foxf1) and other homeobox containing transcription factors (Hoxc4, Ttf-1 and Pcsk5) [23]. Various environmental teratogens have also been implicated in the pathogenesis of OA-TOF [23]. Infants born to mothers with prolonged exposure to contraceptive pills (exposure to progesterone and oestrogen) during pregnancy have high risk. Oesophageal atresia has also been reported in some infants of hyperthyroid and uncontrolled diabetic mothers. Intrauterine exposure to thalidomide and diethylstilbestrol are also found to be associated with OA.

\subsection{Epidemiology of oesophageal atresia}

The incidence of OA, with or without TOF, is reported to be 1:3500 live-born infants. This, however, varies geographically [23] from 1 in 2440 births in Finland to 1 in 4500 births in the United States and Australia. In a European study, 62\% of infants with OA-TOF were male, whereas a California database found considerable variations in the male-to-female ratios between types of OA-TOF defects [23]. Mothers of white ethnicity have a higher $(>60 \%)$ prevalence of OA-TOF than nonwhite populations do [23]. First pregnancy and increasing maternal age have been found to be associated with an increased risk OA-TOF [23]. The risk is reported to be twofold for women 35-40 years old and threefold for those older than this age [23]. Offspring of in vitro fertilization patients also have a significantly increased risk of developing OA (OR 3.65:CI 2/53-5/26) [23].

\subsection{Anatomic (pathologic) classification of oesophageal atresia}

Various systems of classifications have been used to classify OA. The two most frequently used ones are shown in Table 1 [24].

\subsection{Associated anomalies}

About $50-70 \%$ of patients with oesophageal atresia have associated congenital anomalies [23]. Cases without tracheo-oesophageal fistula tend to be most commonly associated with other anomalies, while those with the H-type are less commonly associated with other anomalies. Associated anomalies may negatively affect patient management and overall outcome of patients.

\begin{tabular}{|c|c|c|c|}
\hline Amatomic Descriptioe & Geoss type & Vogt type & $\begin{array}{l}\text { Approvimate inddence } \\
\text { CS41 }\end{array}$ \\
\hline Orsophukeal Agenesis & $=$ & 1 & \\
\hline 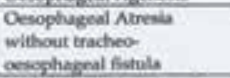 & A & II & 6 \\
\hline $\begin{array}{l}\text { Oesophrageal atresta with } \\
\text { proximal tracheo- } \\
\text { oswogheseal fistula }\end{array}$ & B & itla & 2 \\
\hline $\begin{array}{l}\text { Oesophugesl atresis with } \\
\text { distal trocheo-oesophageal } \\
\text { fasula }\end{array}$ & C & Inb & 85 \\
\hline $\begin{array}{l}\text { Oesophageal atresia with } \\
\text { both provimal and distal } \\
\text { tracheo-cesophugeal } \\
\text { fiatulae }\end{array}$ & D & Itile & 1 \\
\hline $\begin{array}{l}\text { Tracheo-oesophageal } \\
\text { fistula without } \\
\text { oesughegeal atrsia }\end{array}$ & $\mathbf{E}$ & IV & 6 \\
\hline
\end{tabular}

Table 1.

Types of oesophageal atresia-anatomic (pathologic) classification of oesophageal atresia. 
About $50 \%$ of patients with associated anomalies have recognizable syndromes, sequences, and associations [23]. VACTERL (vertebral, anorectal malformation, cardiac, tracheo-oesophageal, renal, limb) association is found in $20 \%$ of cases [25]. These syndromes, sequences, and associations usually result from chromosomal anomalies or single gene mutations (Tables 2 and 3) [23, 26].

Patients with oesophageal atresia have trisomies 18 and 21 in about $7 \%$ of patients. In trisomy 21 (Down syndrome), the possibility of associated duodenal atresia, Hirschsprung's disease and congenital heart disease should be considered. In patients with clinically suspected trisomy 18 , surgery should be postponed and chromosomal analysis done immediately since prognosis for trisomy 18 is very poor.

The common associated anomalies in non-syndromic oesophageal atresia include the cardiovascular system (CVS), genitourinary tract (GUT), gastrointestinal tract (GIT), musculoskeletal system (MSS), and central nervous system (CNS) (Table 4). Cardiovascular anomalies tend to occur most frequently (Table 4) [23, 25, 27]. This is followed by the GUT, GIT, MSS, and CNS (neurologic anomalies). A review of cases seen at our centre in Accra Korle-Bu, however, showed overall incidence of associated anomalies of $32.5 \%$, with GIT system being the most commonly affected, followed by the CVS [9].

The most common CVS anomalies are ventricular septal defect (VSD) and atrial septal defect (ASD). Other CVS defects include tetralogy of Fallot, patent ductus arteriosus (PDA), and coarctation of the aorta. A few patients tend to have the descending thoracic aorta on the right side. Renal agenesis or hypoplasia, undescended testis, cystic renal disease, hydronephrosis, vesicoureteric reflux (VUR) and ureteric duplication are some of the GUT anomalies associated with oesophageal atresia. Other GUT anomalies include pelvi-ureteric junction (PUJ) obstruction, vesicoureteric junction (VUJ) obstruction, urachal anomalies, ambiguous genitalia, bladder exstrophy and cloacal exstrophy. The GIT anomalies found in oesophageal atresia include anorectal malformation (ARM), duodenal atresia, intestinal malrotation, ileal atresia, annular pancreas and pyloric stenosis. Vertebral and radial abnormalities are the usual MSS defects associated with oesophageal atresia. The CNS defects found in oesophageal atresia include neural tube defects, hydrocephalus, holoprosencephaly and anophthalmia or microphthalmia.

\subsection{Pathophysiology of oesophageal atresia}

The discontinuation of the oesophageal lumen prevents swallowed amniotic fluid by the foetus from reaching the stomach and hence the intestine. In view of this, the physiological control of amniotic fluid volume by the foetus, through swallowing and absorption, is impaired, leading to polyhydramnios. This would in turn lead to premature rupture of membrane, umbilical cord prolapse and premature delivery.

\begin{tabular}{|c|c|}
\hline Chromosoenal Defect & Resaltunt Syndrome \\
\hline Trisomy 21 & Down syndeome \\
\hline Trisomy 18 & Edward syndrome \\
\hline Trisomy 13 & Patati syndrume \\
\hline Chromosome $22 q$ deletion & $\begin{array}{l}\text { 1. DiCeorge syndrome } \\
\text { 2. Autosomal dominant Opitz G/BBB syndrome }\end{array}$ \\
\hline Chromosome $17 q$ deletion & $\begin{array}{l}\text { Chromosome } 17 q \text { deletion syndrome, including renal } \\
\text { cyst and diabetes (RCAD) syndrone and Mayer- } \\
\text { Rokitansky-Kusber-Hauser syndrome }\end{array}$ \\
\hline Chromosome 16q deletion & $\begin{array}{l}\text { Chromosome } 16 \mathrm{q} \text { deletion syndrome, induding Townes- } \\
\text { Brocks syndrome }\end{array}$ \\
\hline Chromosome $13 q$ deletion & Chromosome 13 g deletion syndrome \\
\hline
\end{tabular}

Table 2.

Chromosomal abnormalities associated with oesophageal atresia. 


\begin{tabular}{|c|c|}
\hline Single Gene Matation (Chromosomal location) & Resultant Syndrome, Sequence and Associations \\
\hline MYCN (2p24.1) & $\begin{array}{l}\text { Feingold syndrome (ODFD syndrome - Oculo-digito- } \\
\text { esophageal-duodenal syndrome) }\end{array}$ \\
\hline CHD7 (Bq12) & $\begin{array}{l}\text { CHARCE (Coloborna, Heart defects, Atresia of nasal } \\
\text { choanae, Retarded growth/development, Genitositinary } \\
\text { abnormalities. Ear anomalies) syndronve }\end{array}$ \\
\hline $50 \times 2(3926.33)$ & AEG (Anolphthalmos-Esophugeal-Cenital) syndtome \\
\hline GL13 (7:13) & Pallister-Hall syndrome \\
\hline FANCA $(16 \mathrm{q} 24.3)$ & Fancont ansemia \\
\hline IBXS (12924.21) & Holv-Oram syndroene (Heart-hand syndroene) \\
\hline Multsfactorial & $\begin{array}{l}\text { Goldenhar syndrome (Otulo-asuriculo-vertebral } \\
\text { syndrome) }\end{array}$ \\
\hline RFX6 $(69221)$ & Martinex-Frias syndrome \\
\hline 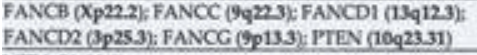 & $\begin{array}{l}\text { VACTERL, Assodiation with hydrocephalus (VACTERL- } \\
\text { H) }\end{array}$ \\
\hline MIDi $\left(x_{p 22.2)}\right.$ & $\mathrm{X}$-linked Opitz G/BBB \\
\hline
\end{tabular}

Table 3.

Single gene mutations associated with oesophageal atresia.

Postnatally, the neonate is not able to swallow saliva and food. This leads to accumulation of saliva and food in the upper oesophageal segment (pouch). The accumulated saliva and food then spill over into the lungs through the larynx and trachea. Patients are therefore prone to the development of aspiration pneumonia. Patients with distal tracheo-oesophageal fistula also tend to aspirate gastric secretions into the lungs, especially if lying in a head-down position.

The distal tracheo-oesophageal fistula also allows air to escape from the trachea to the stomach and hence the intestines. Consequently, patients can develop gross distention of the stomach and intestine, especially if patient is resuscitated using an Ambu bag. The distended stomach can then splint the diaphragm, impairing diaphragmatic excursion and thereby causing respiratory distress. The distended stomach can also rupture leading to chemical peritonitis.

In patients with delayed diagnosis, the constant regurgitation of swallowed food leads to malnutrition. Such patients tend to have poor surgical outcome and overall survival.

Associated congenital anomalies, especially severe cardiac and renal anomalies, also affect management and survival of patients. Low birth weight that may result from premature delivery owing to polyhydramnios also tends to affect management and survival.

\subsection{Clinical presentation}

Oesophageal atresia may be detected prenatally or postnatally.

\subsubsection{Prenatal presentation and diagnosis}

Prenatal diagnosis helps in planning of delivery and possible prenatal referral to centres that have the facilities to manage such patients.

Oesophageal atresia should be suspected prenatally in patients with evidence of polyhydramnios. Polyhydramnios will present with symphysio-fundal height

\begin{tabular}{|l|l|}
\hline Associated anomaly & Incidence (76) \\
\hline CVS & 24 \\
\hline CUT & 21 \\
\hline CIT & 21 \\
\hline MSS & 14 \\
\hline CNS & 7 \\
\hline
\end{tabular}

Table 4.

Common associated anomalies in non-syndromic oesophageal atresia. 
of more than the expected for gestational age. This is confirmed with antenatal ultrasonography with amniotic fluid index (AFI) of more than $24 \mathrm{~cm}$.

Antenatal ultrasonographic features of polyhydramnios, a small stomach, a distended upper oesophageal pouch and abnormal swallowing should raise the suspicion of oesophageal atresia [28-31].

\subsubsection{Postnatal presentation and diagnosis}

\subsubsection{History}

Postnatally, patients with oesophageal atresia present with drooling of saliva (excessive salivation). A history of antenatal polyhydramnios gives more credence to the diagnosis of oesophageal atresia. Delayed diagnosis leads to aspiration of saliva from the upper oesophageal pouch, causing aspiration pneumonia with cough, cyanosis and fever. Feeding leads to worsening of the aspiration pneumonia. Hence, early detection and avoidance of oral feeds helps to promote good outcome of patients.

Patients with distal tracheo-oesophageal fistula are also at risk of aspiration of gastric secretions into the lungs. In addition, swallowed air passes through the distal tracheo-oesophageal fistula into the stomach. This can lead to gross gastric distention, especially following Ambu bagging, resulting in diaphragmatic splinting and sometimes gastric rupture. They may thus present with respiratory distress, gross abdominal distention and sometimes peritonitis.

Patients with tracheo-oesophageal fistula without oesophageal atresia are usually diagnosed late. They present with recurrent cough associated with feeding and recurrent episodes of pneumonia.

Other symptoms would depend on associated congenital anomalies. Patients with cardiac anomalies may present with cyanosis. Patients with renal agenesis will have a history of anuria.

During history taking, one should seek for a maternal history of uncontrolled diabetes mellitus and the use of drugs such as oral contraceptives, antithyroid drugs (carbimazole and methimazole) and thalidomide. These are risk factors for oesophageal atresia.

The history of the maturity and weight of the baby at birth are also important considerations.

\subsubsection{Physical examination}

The principles of physical examination of a patient with suspected oesophageal atresia are to confirm the diagnosis, assess for aspiration pneumonia and evaluate for associated congenital anomalies.

To confirm the diagnosis, a stiff radiopaque 10-gauge French catheter is passed through the mouth into the oesophagus. The tube is not passed through the nose because it may traumatize the nasal passages. The diagnosis of oesophageal atresia is heightened if the tube fails to reach the stomach by getting arrested in the proximal oesophageal pouch at about $10 \mathrm{~cm}$ from the alveolar margin. A soft and smaller tube may coil in the upper oesophageal pouch, giving an impression of reaching the stomach. The secretions sucked through the tube can be tested with litmus paper to differentiate between saliva (basic) in the upper oesophageal pouch and gastric secretions (acidic).

The presence of fever, respiratory distress, reduced air entry in the lungs (especially on the right side) and crepitations in the lungs are an indication of aspiration pneumonia. However, patients in heart failure will have similar chest findings, and 
these are usually bilateral. Gastric distention and gastric rupture will be associated with respiratory distress, abdominal distention and abdominal signs of peritonitis.

Meticulous examination for associated anomalies is done. The presence of cyanosis may be due to cyanotic heart disease. The chest is examined for heart murmurs and crepitations. The abdomen is examined for any palpable flank mass. The perineum is examined for ARM. The spine and the limbs are also examined for any anomaly. Indeed, the VACTERL association could be used as a guide to take history, examine and investigate for any associated congenital anomaly.

\subsubsection{Investigations}

Investigations are also done to confirm the diagnosis, to assess for complications (aspiration pneumonitis and gastric rupture) and to evaluate for associated anomalies.

Oesophageal atresia is confirmed by taking a plain X-ray of the neck, chest, and abdomen with a radiopaque tube passed through the mouth in situ. In the presence of oesophageal atresia, the X-ray will show the tube arrested in the upper oesophageal pouch or coiled in the upper pouch. The same X-ray is also evaluated for evidence of associated aspiration pneumonia, cardiac enlargement, dextrocardia and vertebral anomalies.

The abdominal portion will help to determine the presence of air in the stomach and intestine. The presence of air in the stomach and intestine indicates the presence of a distal tracheo-oesophageal fistula. Pneumoperitoneum indicates gastric rupture. A gasless abdomen implies the absence of a distal tracheo-oesophageal fistula. Such a patient may either have no tracheo-oesophageal fistula or a proximal fistula. The proximal fistula can be identified with the aid of bronchoscopy [32]. Table 5 summarises the important diagnostic features of OA.

Abdominal ultrasound to assess the kidneys, ureters and the urinary bladder is also done. Patients with bilateral renal agenesis or severe multicystic dysplastic kidneys will need no further management since such patients will not survive. Renal scintigraphy will confirm non-functioning kidneys.

Ultrasonography (USG) of the spine is used to assess the spinal cord for anomalies, including tethered cord. The USG of the spinal cord should be done before 3 months of age since the bones become ossified after this age, rendering the use of USG impossible. Magnetic resonance imaging (MRI) may be used to evaluate the spinal cord. It is, however, very expensive and implies the need for general anaesthesia in a new born with a relatively high anaesthetic risk. It is not readily available at most centres, especially in the developing countries.

\begin{tabular}{|c|c|c|}
\hline & Prenatal & Postnatal \\
\hline Clinical Features & - Rolyhydramnios & 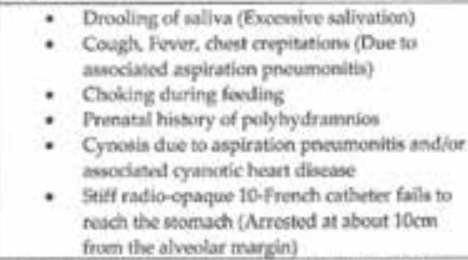 \\
\hline Imaging Features & $\begin{array}{l}\text { Ultrascond: } \\
\text { - Dilatod upper } \\
\text { oesophageol pouch } \\
\text { - Small stumiach } \\
\text { - Abnormal wallowing }\end{array}$ & 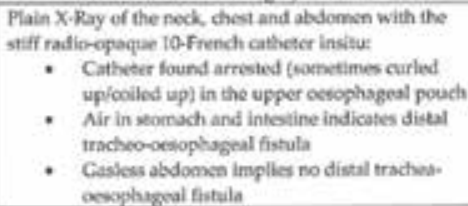 \\
\hline
\end{tabular}

Table 5 .

Important diagnostic features of oesophageal atresia. 
Echocardiogram is used to evaluate the heart for a congenital heart disease and the direction of the arch of the aorta. The direction of the arch of the aorta (normally to the left) is utilised in deciding the side of the thoracotomy incision (usually on the right side since the arch is normally to the left). At our centre, echocardiogram is not routinely done for every patient since it is expensive and not readily available. Hence, we selectively use it for only patients with clinical evidence of congenital heart disease.

Patients with suspected chromosomal and genetic anomalies are evaluated to confirm the anomaly. However, this is not available at most centres in developing centres, including our centre. Patients with confirmed trisomy 18 (Edwards syndrome) should have any planned surgical intervention abandoned since prognosis for these patients is very poor.

Patients with Down syndrome should be evaluated for the possibility of associated duodenal atresia, congenital heart disease and Hirschsprung's disease.

Other investigations that are supportive are full blood count (FBC) and blood urea, electrolytes, and creatinine (BUE\&Cr). Blood gases may be analysed in patients who are critically ill.

\subsection{Clinical prognostic classification of patients}

Prognostic classification is done based on risk factors that affect survival of infants with OA. It is used to guide operative treatment and to compare case outcomes over time and between centres. The first prognostic risk stratification of patients with OA was developed in 1962 (Waterston classification) [4]. This is based on birth weight, the presence of pneumonia and associated congenital anomalies (Table 6). It has provided important contribution to the care of infants with OA. Patients in group A (good-risk category) are offered immediate primary repair of the defect. Those in group B (moderate-risk category) are treated with delayed primary repair, while those in group $\mathrm{C}$ (high-risk category) are managed by staged repair.

Several new classification schemes have been developed because of improvement in neonatal intensive care and availability of more treatment options for multiple congenital anomalies. These new schemes include refinement of the Waterston classification by Randolf and colleagues in 1989 [33]. This is based on the overall physiologic status of the patient. Poenaru et al. [34] also developed a new prognostic classification based on severe pulmonary dysfunction with preoperative mechanical ventilation and severe associated anomalies. Spitz et al. [35] developed a new risk classification based on birth weight and major cardiac anomaly (Table 7). Currently, the Spitz classification is the most commonly used system $[36,37]$. Another new prognostic classification system has been developed by adding preoperative respiratory distress syndrome and pneumonia to the Spitz classification [38].

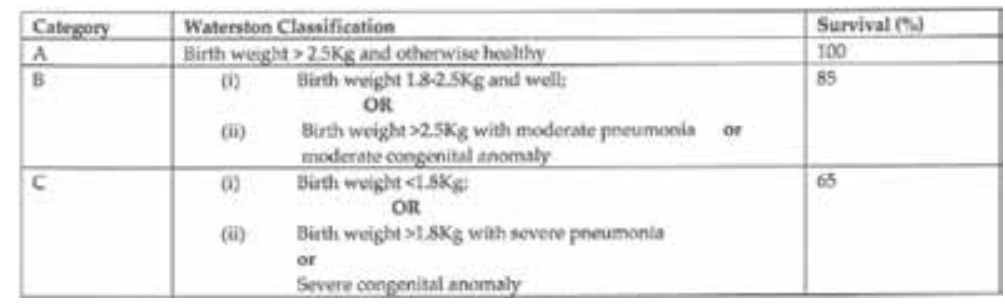

Table 6.

Waterston prognostic classification of oesophageal atresia infants. 


\begin{tabular}{|c|c|c|}
\hline Group & Spitz Classification & Survival Fa \\
\hline 1 & $\begin{array}{l}\text { Burth weight }>1.5 \mathrm{Kg} \text { without majar } \\
\text { owegenilat hoart disosse }\end{array}$ & 97 \\
\hline ii & $\begin{array}{l}\text { Dirth weight } \angle 15 \mathrm{Kg} \text { OR Mtojor } \\
\text { congenital heart diowne }\end{array}$ & 59 \\
\hline iil & 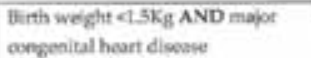 & 22 \\
\hline
\end{tabular}

Table 7 .

Spitz prognostic classification of infants with oesophageal atresia.

\subsection{Management of patients}

\subsubsection{Pre-operative management}

The aim of preoperative management is to make patient stable before surgical management of the oesophageal atresia. This involves prevention of complications, treatment of life-threatening complications, management of life-threatening associated congenital anomalies and general supportive measures.

Complications to be prevented are mainly aspiration and diaphragmatic splinting by gaseous distention of the stomach and intestines. Maintaining the child in a partly upright position and by repeated or continuous suctioning of the upper oesophageal pouch prevents aspiration. These measures keep the proximal oesophagus empty and reduce the likelihood of overflow of saliva into the lungs. The repeated suctioning of the upper oesophageal pouch should be done every 10 min or more often if the child appears to have excessive mucus or air bubbles. The best tube for suctioning, especially for continuous suctioning, is the Replogle tube. The partial upright position prevents aspiration of stomach secretions. Infants with OA should not be fed to prevent aspiration. The child should have minimal handling to prevent excessive crying and consequent filling of stomach with air. In addition, vigorous resuscitation by Ambu bagging should be avoided to prevent gastric distention.

The main life-threatening complication is respiratory distress. Respiratory distress may be due to prematurity, other congenital abnormalities, aspiration pneumonia, or diaphragmatic splinting. Diaphragmatic splinting in patients with OA results from excessive escape of air through the distal fistula into the stomach, causing distention of the stomach and intestines and, in some cases, causing gastric rupture. Thorough evaluation of the infant to determine the underlying cause and prompt management is paramount. Gross gastric distension and perforation, causing pneumoperitoneum and elevation of the diaphragm, can cause major morbidity by worsening ventilation. Prompt needle decompression of the abdomen should be offered. This is followed by urgent laparotomy to control the air leakage. This involves insertion of a Foley catheter through the gastric perforation into the lower oesophagus, thereby occluding the distal TOF and allowing thoracotomy to proceed, at which time the distal TOF is divided. Blood gases should be monitored, but if monitoring facilities are not available, the infant must be kept pink at all times, and pulse oximetry for monitoring is considered standard.

Patients with duct-dependent congenital heart disease should be stabilised, by keeping the duct (patent ductus arteriosus) patent with prostaglandin E1 infusion, before surgery is done.

General supportive measures include keeping the child warm, vitamin $\mathrm{K}$ administration, provision of IV fluids/parenteral nutrition and administration of broad-spectrum antibiotics. 


\subsubsection{Operative management of oesophageal atresia}

The operative management may be immediate primary repair, delayed primary repair or staged repair; and this depends on the prognostic category of the patient and the gap between the upper and the lower oesophageal segments. Patients with pneumonia and duct-dependent congenital heart disease need to be stabilised and planned for delayed primary repair. Patients with long-gap oesophageal atresia could also be managed with delayed primary repair. Further growth of the upper oesophageal pouch tends to occur if primary repair is delayed for about 3 months. Hence, the role of total parenteral nutrition (TPN) becomes significantly important. No consensus has been reached for the definition of long-gap OA. However, long gap is defined by some authorities as a gap of $\geq 3$ vertebral bodies or $\geq 5 \mathrm{~cm}$ [39].

Primary repair is achieved mostly through a right thoracotomy (readers should read operative textbooks for details of the surgical procedure). However, in patients with right-sided arch of aorta, left thoracotomy is done. The patient is placed at the right side uppermost and with a towel folded underneath the left chest to give lateral flexion. The right arm is extended above the head and the head slightly flexed. A transverse incision is centred on the inferior angle of the scapula, and the chest is entered through the fourth intercostal space. Approach to the oesophagus may be extrapleural or transpleural. The extrapleural approach is favoured because of the less likelihood of developing empyema following an anastomotic leak. The pleura is swept off the chest wall to identify the structures of the posterior mediastinum. The azygous vein is then ligated and divided.

Any distal TOF is identified and divided close to the trachea. The fistula is then closed with interrupted absorbable sutures such as 4/0 Vicryl, PDS, or Monocryl. Care should be taken to avoid damage to the vagal fibres and blood supply to the distal oesophageal segment.

The upper oesophagus is identified with the aid of a tube passed through the mouth or nose by the anaesthetist. It is then mobilised enough to allow an end-toend, one-layer, interrupted oesophageal anastomosis, ensuring that the mucosa and the submucosa are included. The upper oesophageal segment can be extensively mobilized along its full length without risk of significant ischemia. If extensive mobilization of the proximal oesophagus fails to provide adequate length, the lower oesophagus may be mobilized to prevent undue tension on the anastomosis. Mobilising the lower oesophagus without complete disruption of its segmental vascular supply is possible. However, care should be taken to avoid excessive or rough handling of the oesophagus. If, despite extensive mobilisation of the two oesophageal segments, an anastomosis cannot be performed without excessive tension, an oesophageal myotomy (Levaditis procedure) can be performed. Myotomy (circular or spiral myotomy) is usually done using the upper pouch. It can cause significant damage to the nerve and blood supply distal to the myotomy and predisposes to diverticulum formation and strictures.

Patients with TOF without atresia have an oblique fistula running downward from the trachea to the oesophagus, usually at the level of T1-T3. This level is somewhat higher than what is seen in most patients with oesophageal atresia. In these patients, a cervical approach provides the best surgical access as most fistulas are in the root of the neck (at about the level of the second thoracic vertebra). Care should be taken to avoid damage to the recurrent laryngeal nerves, which lie in the grooves between the oesophagus and the trachea and closely related to the fistula, during operative dissection.

Staged repair of OA involves division of TOF, placement of feeding gastrostomy and cervical oesophagostomy. Disconnection of distal TOF is done at most centres through thoracotomy. At our centre, however, this is done by dividing the abdominal 
oesophagus during the placement of the feeding gastrostomy [27]. This helps to avoid the stress of thoracotomy that may negatively affect patient outcome. Patients who undergo staged repair are later offered oesophageal replacement surgery.

It should be noted that the surgical management of OA has been advanced into the realm of minimally invasive surgery (thoracoscopy) due to recent advances in surgical techniques. [40-49]. Thoracoscopic approach to the repair of OA is associated with early recovery and minimal chest wall musculoskeletal morbidity as compared with open surgery (thoracotomy).

\subsection{Manoeuvres for managing long-gap OA to achieve primary repair}

Various manoeuvres are used to narrow the gap between the upper and lower oesophageal segments [50]. These manoeuvres are classified as preoperative manoeuvres and intraoperative manoeuvres (Table 8).

Preoperative manoeuvres are those that are done before the surgery for primary repair is attempted; and they may be achieved thoracoscopically [45, 51]. These include external traction technique by Foker [50, 52, 53]; multistage, extrathoracic elongation technique by Kimura [52, 54]; bougienage of the upper oesophageal pouch, sometimes including the lower pouch; placement of magnets in the two ends of the oesophageal segments with patient placed in an electromagnetic field and delaying of surgery for some months to allow growth of the oesophagus.

In the Kimura technique, the upper part of the oesophagus is mobilised and brought out as an end-cervical oesophagostomy. The oesophagus and its cutaneous stoma are surgically mobilised and translocated down the anterior chest wall every 2-3 weeks. This is continued until enough length is achieved to perform an end-to-end oesophageal anastomosis. The Foker technique involves open or thoracoscopic placement of traction sutures on both the proximal and distal oesophageal pouches with the sutures exiting through the chest wall. These sutures are serially pulled in opposite directions until the pouches approximate. This external traction technique of Foker is reported to induce oesophageal growth and expedite approximation of the pouches.

Internal traction techniques have also been used to bridge long gaps [45, 55]. These include open or thoracoscopic suturing of the oesophageal segments to the prevertebral fascia or costal bone under tension.

Intra-operative manoeuvres include full mobilisation of the upper segment of the oesophagus; mobilisation of the distal segment; circular myotomy (Levaditis technique) or spiral myotomy, usually of the upper pouch and mobilisation of the stomach into the chest.

Other intraoperative techniques include full-thickness anterior flap of the upper pouch [56] and injection of Botox into the upper segment.

Figure 3 shows an algorithm for the management of patients with oesophageal atresia.

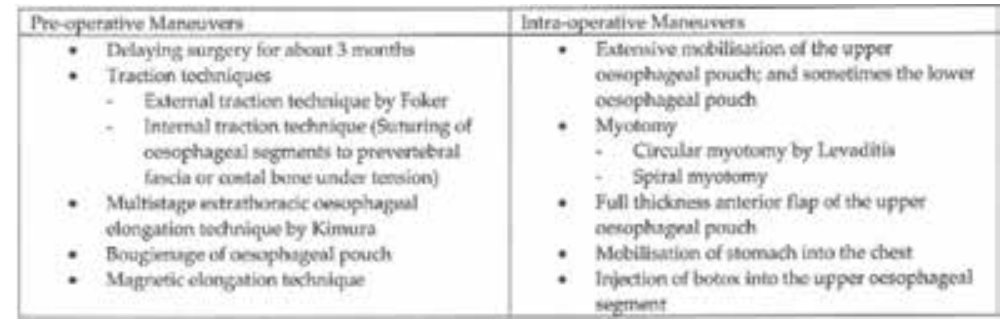

Table 8.

Pre-operative and intraoperative oesophageal elongation manoeuvres for long-gap oesophageal atresia. 


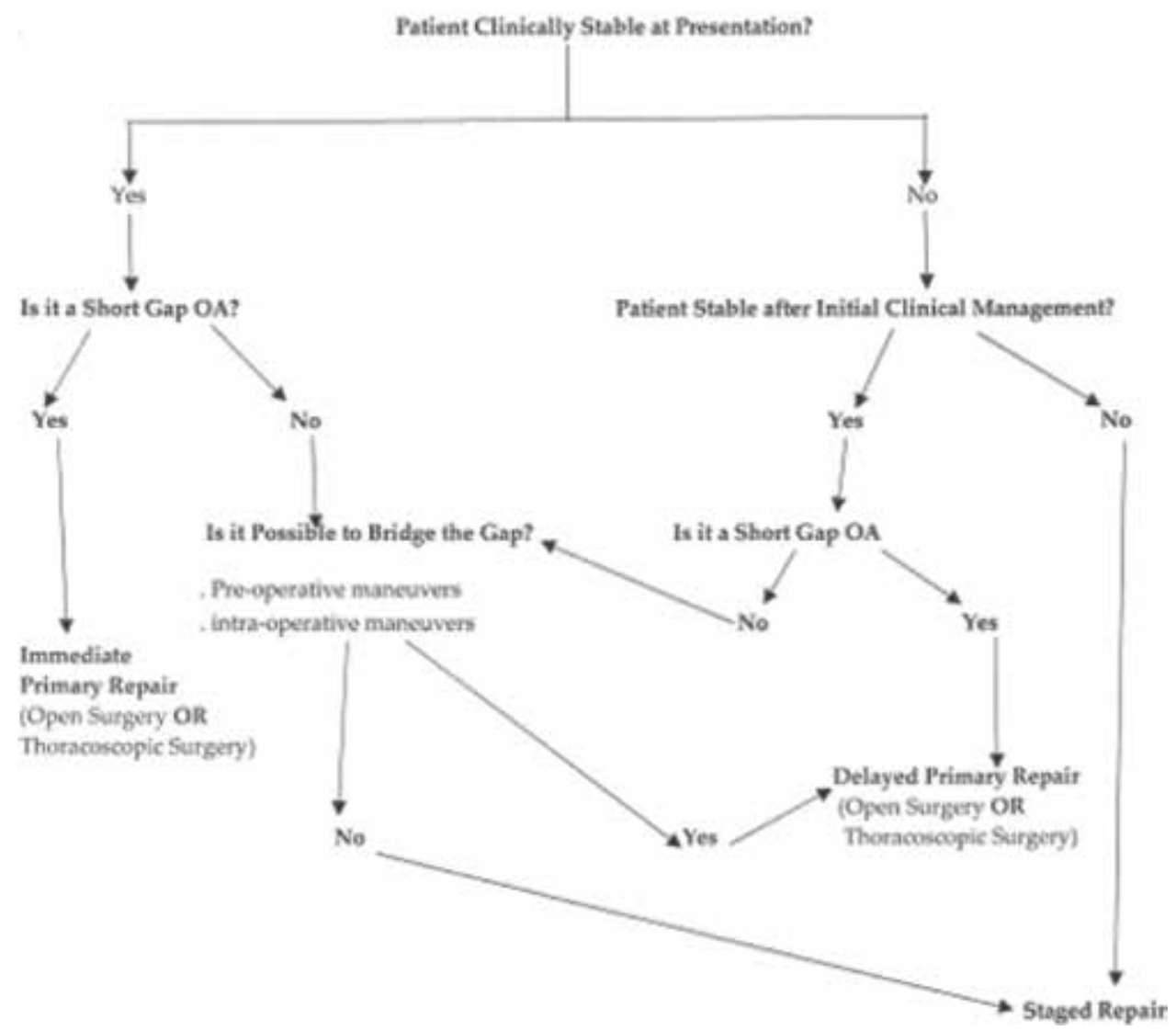

Figure 3.

Algorithm for the management of patients with oesophageal atresia.

\subsection{Complications after repair of OA}

Complications [57-59] resulting from repair of OA are generally grouped into two: early and late complications (Table 9). Early complications include anastomotic leak, anastomotic stricture, and recurrent tracheo-oesophageal fistula. Tracheomalacia, gastro-oesophageal reflux and oesophageal dysmotility are the late complications. Factors that promote postoperative complications include preoperative intubation, birth weight less than $2.5 \mathrm{~kg}$, long-gap OA [60], post-operative intubation for more than 4 days, anastomotic leak and inability to feed orally for more than 1 month [61]. Management of the complications may involve a multidisciplinary approach.

\subsubsection{Early complications}

\subsubsection{Anastomotic leak}

Anastomotic leak at the oesophago-oesophagostomy is found in about $14-16 \%$ of patients after primary repair of OA. Most often, the leaks are clinically insignificant and can be managed with adequate drainage and nutritional support. Up to $95 \%$ of the leaks close spontaneously when a retropleural approach is undertaken and a patent mediastinal drain is in place [62]. Even in transpleural repair with leakage, spontaneous closure occurs with adequate drainage. Anastomotic breakdown 


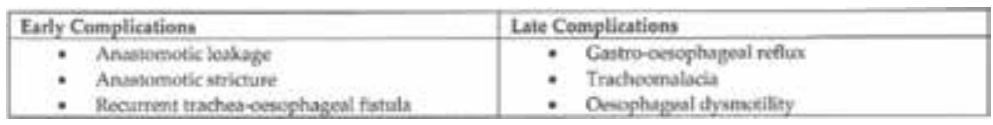

Table 9.

Complications associated with oesophageal atresia repair.

usually leads to the formation of a stricture at the site and may be associated with a recurrent TOF. Only 3-5\% of anastomotic leaks are known to result from major disruptions of the oesophageal anastomosis. They are found to be typically seen within 24-48 h after repair. Patients usually deteriorate as result of tension pneumothorax or mediastinitis. Hence, prompt reoperation with adequate drainage is imperative. Repair of the leak may be attempted, and this may be buttressed with the help of a pleural or pericardial patch, with or without intercostal muscle flap. Contributing factors to anastomotic leak include poor surgical technique, ischemia of the oesophageal ends, the use of myotomy and excessive tension at the anastomotic site $[63,64]$. If reanastomosis is not possible, cervical oesophagostomy and delayed oesophageal replacement would be required.

\subsubsection{Anastomotic stricture}

Anastomotic stricture is found to be a common complication after repair of $\mathrm{OA}$. It is characterised by dysphagia and recurrent respiratory problems due to aspiration or foreign body obstruction. The narrowing is noted on endoscopy or contrast oesophagography. Poor anastomotic technique (excessive tension, twolayered anastomosis and silk suture material), long gap, ischemia at the ends of the oesophagus, gastro-oesophageal reflux and anastomotic leak are factors implicated in the pathogenesis of oesophageal stricture.

Anastomotic stricture is treated by dilatation. However, a stricture resistant to repeated dilatations will require resection and reanastomosis or oesophageal replacement. Triamcinolone injection may be used at the stricture site. However, repeated injections may lead to adrenal suppression. Application of mitomycin $\mathrm{C}$ to the stricture under endoscopic control has also been reported to reduce stricture formation after dilation. It is important to determine whether the oesophageal stricture is associated with gastro-oesophageal reflux. This can be determined using contrast oesophagography, endoscopy, $\mathrm{pH}$ monitoring or a combination of these studies. The presence of gastro-oesophageal reflux is initially managed medically with proton pump inhibitors. Failure of medical management may warrant antireflux procedure.

\subsubsection{Recurrent tracheo-oesophageal fistula}

Recurrent TOF commonly results from anastomotic leak with local inflammation and erosion through the previous site of TOF repair. Recurrent TOF can be minimised by the use of a pleural flap, vascularized pericardial flap or azygous vein flap interposed between the oesophageal and tracheal suture lines. Symptoms of recurrent TOF can be typical of those seen with a congenital H-type TOF (coughing with feedings and recurrent respiratory distress). However, less obvious symptoms such as recurrent pulmonary infections are more common. Air-filled oesophagus on plain radiographs of the chest is suggestive of the diagnosis. As done in patients with congenital $\mathrm{H}$-type fistula, contrast oesophagography performed in the prone position under videofluoroscopy is a reliable method of establishing the diagnosis. Another reliable diagnostic approach is bronchoscopy with cannulation of the fistula with a 2- to 3-French catheter. It is invaluable in locating the fistula 
during the operative procedure. About $50 \%$ of recurrent TOF is missed on routine contrast swallow studies. A recurrent TOF rarely closes spontaneously and usually requires surgical repair. Operation of choice is thoracotomy with fistula ligation, and division is the operation of choice. Pleura, intercostal muscle or pericardium should be interposed between the oesophagus and trachea to minimise recurrence. Endoscopic treatment of TOF by means of various chemicals or diathermy has also been reported. Various case reports and case series have reported the use of diathermy or laser deepithelialization followed by fibrin glue.

\subsubsection{Late complications}

\subsubsection{Gastro-oesophageal reflux}

Gastro-oesophageal reflux is a common complication after repair of OA [65]. It is probably related to shortening of the intra-abdominal portion of the oesophagus because of anastomotic tension and/or oesophageal motor dysfunction. The motor dysfunction may be intrinsic to the congenital anomaly or acquired from operative manipulation. Clinically, gastro-oesophageal reflux is suspected in patients with symptoms of vomiting, dysphagia and recurrent anastomotic stenosis. Episodes of foreign body or food bolus impaction may occur. Respiratory symptoms such as stridor, cyanotic spells, recurrent pneumonia and reactive airway disease are also suggestive of gastro-oesophageal reflux.

Upper gastrointestinal contrast study and 24-h pH probe data are diagnostic tools for gastro-oesophageal reflux. Multichannel oesophageal impedance combined with $\mathrm{pH}$ monitoring may emerge as a superior test. Abnormal oesophageal peristalsis and decreased lower oesophageal sphincter pressures after OA repair have been documented on oesophageal manometry. Medical management typically consists of thickening of feedings, positioning of the infant in a prone or upright posture, administration of acid reduction agents such as histamine- 2 blockers, proton pump inhibitors and prokinetic agents. Antireflux operations are offered for patients with failed medical management, failure to thrive, chronic pulmonary infection, refractory anastomotic stricture or the development of a distal oesophageal stricture.

\subsubsection{Tracheomalacia}

Respiratory symptoms occurring after repair of OA can be due to tracheomalacia. Tracheomalacia is defined as generalized or localized weakness of the trachea that allows the anterior and posterior tracheal walls to come together during expiration or coughing.

Symptoms of tracheomalacia are often difficult to clinically distinguish from those of recurrent TOF, anastomotic leak, or gastro-oesophageal reflux. Embryologic events leading to TOF are believed to contribute to the development of tracheomalacia [61]. The tracheal cartilage is shorter than normal, thereby failing to provide the support necessary to maintain a patent airway [61]. The trachea may also be compressed between the aorta anteriorly and the often dilated upper oesophagus posteriorly after repair of OA; and such compression has been considered a significant contributor to the pathophysiology of tracheomalacia [61]. The tracheal collapse usually occurs in the region of or just above the original site of TOF in the distal third of the trachea which is generally at the level of the aortic arch; and severe tracheomalacia appears less common in infants with pure OA. Tracheomalacia has broad clinical manifestations, ranging from a "brassy" or "barking" cough in mild cases to recurrent pneumonia or acute, life-threatening 
apnoeic spells. Because of difficulty in breathing or cyanotic attacks during feeding, infants with tracheomalacia are often reluctant to feed. Life-threatening apnoeic and cyanotic spells occur during or within 5-10 min of a meal. They are characterized by cyanosis progressing to apnoea, bradycardia, and ultimately, cardiorespiratory arrest if not detected and managed promptly. Diagnosis is established by bronchoscopy with spontaneous ventilation. This reveals a slit-like lumen of the trachea at the involved area. However, because the symptoms overlap those of a stricture or gastro-oesophageal reflux, contrast oesophagogram is usually done as an initial investigation. Close attention to the tracheal air column on the lateral views during such a study will often reveal complete tracheal collapse during forced expiration (i.e., crying) or when contrast fills a distended upper oesophagus just above the anastomosis. Most mild to moderate symptoms of tracheomalacia tend to improve with time. Hence, operative intervention is not required. Operative treatment of choice for patients with severe symptoms, including acute life-threatening events, is aortopexy $[46,61,66]$. This is usually performed through a left anterior mediastinotomy (Chamberlain approach) or anterolateral thoracotomy [61]. The ascending aorta and arch are sutured to the posterior surface of the sternum after partial thymectomy [61]. The lifting of the aorta up in this fashion raises the anterior wall of the trachea and opens the tracheal lumen. In cases in which the aortic arch would not reach the posterior aspect of the sternum without undue tension, the use of a flap of pericardium based at the root of the aorta to be sutured to the sternum may be used [61]. Aortopexy and tracheopexy have also been done through anterior mediastinal approach via a low transverse cervical incision.

\subsection{Oesophageal replacement}

Oesophageal replacement surgery is usually done for patients with OA when primary repair fails or when primary repair is impossible. Various operative procedures have been described; and the most commonly used ones are colon or ileocolon interposition, reversed (antiperistalsis) gastric tube interposition, isoperistalsis gastric tube interposition, jejunum interposition and gastric transposition (gastric pull-up) [67] (Table 10).

Among the methods, colon replacement, or ileocolon, has been widely practiced for many years as a method of oesophageal replacement. This involves placement of the right or left colon substernally or behind the hilum of the lung on the right or left side. To avoid stricture or ulceration at the cologastric anastomosis, vagotomy and a gastric drainage procedure are typically performed. Complications after colonic interposition include cervical anastomotic leak, stricture and intrathoracic redundant colon with stasis, gastric reflux, respiratory problems and diarrhoea.

Reversed gastric tube as a substitute is preferred by some surgeons. A tubularised portion of the greater curvature is brought up to the cervical oesophagus in the substernal or retrohilar position. This procedure has similar complications as described for colonic interposition. A portion of the greater curvature of the stomach can be fashioned into a "free" tube graft based on the right gastroepiploic artery; and this is used as a modification of the reversed gastric tube for oesophageal replacement.

- Colon or flencolon interponition

- Gontric tube interposition Reversed (antiperialaleis)

Isoperiatalkis

- Jofanum interposition

- Castric tranespoition (gastric pull-up?

Table 10.

Commonly used oesophageal replacement techniques. 
Oesophageal replacement can also be achieved using the jejunum, both in a Rouxen-Y fashion and as a free graft with microvascular anastomosis. Recently, a wellestablished method for oesophageal replacement is the use of gastric transposition.

\subsection{Outcome and conclusion}

During the early years, the surgical management of OA was associated with lots of challenges and high mortality [1-4]. Respiratory failure, inadequate resuscitation, and complications of prematurity resulted in most deaths in the past. Complications of the surgical repair of the oesophageal atresia itself, particularly sepsis after dehiscence of the oesophageal anastomosis, and prolonged poor nutrition are other major causes of mortality.

Over the past two to three decades, however, the surgical outcome has improved significantly in most centres in the developed countries. This improvement is attributed to advances in neonatal anaesthesia, well-established neonatal intensive care units (NICU), availability of total parental nutrition (TPN) and refined surgical skills $[1,5-8]$. The current major cause of mortality in most developed countries is from associated major congenital abnormalities. Death from prematurity or oesophageal complications is now rare. In view of this, the previously used Waterston classification has little relevance in developed countries. The prognosis however remains poor in developing countries where late presentation is the norm. Waterston classification may remain relevant in these countries.

Gastro-oesophageal reflux and poor oesophageal clearance due to some degree of ongoing oesophageal dysmotility may limit long-term survival. Dysplastic changes in the lower oesophageal mucosa may predispose to oesophageal carcinoma. Ongoing gastro-oesophageal reflux is a significant risk fact for oesophageal carcinoma. Hence, regular surveillance in these patients is important.

\section{Conflict of interest}

Nil.

\section{Author details}

Samuel Osei-Nketiah* and William Appeadu-Mensah

Department of Surgery, University of Ghana School of Medicine and Dentistry, Accra, Ghana

*Address all correspondence to: oseivatican@yahoo.com

IntechOpen

(C) 2019 The Author(s). Licensee IntechOpen. This chapter is distributed under the terms of the Creative Commons Attribution License (http://creativecommons.org/licenses/ by/3.0), which permits unrestricted use, distribution, and reproduction in any medium, provided the original work is properly cited. (cc) BY 


\section{References}

[1] Pinheiro PF, Simões e Silva AC, Pereira RM. Current knowledge on esophageal atresia. World Journal of Gastroenterology. 2012;18:3662-3672

[2] Spitz L. Oesophageal atresia. Orphanet Journal of Rare Diseases. 2007;2:24

[3] Lanman TH. Congenital atresia of the oesophagus: A study of thirty-two cases. Archives of Surgery. 1940;7:1060

[4] Waterston DJ, Carter RE, Aberdeen E. Oesophageal atresia: Tracheooesophageal fistula. A study of survival in 218 infants. Lancet. 1962;1:819-822

[5] Spitz L. Esophageal atresia. Lessons I have learned in a 40-year experience. Journal of Pediatric Surgery. 2006;41:1635-1640

[6] Grosfeld JL, Ladd AP. Congenital anomalies. In: Silva AC, Pereira RM, Pinheiro PF, editors. Pediatric SurgeryClinical and Surgical Conduct. Rio de Janeiro: Guanabara Koogan; 2005. pp. 291-298

[7] Nakayana DK. Congenital abnormalities of the esophagus. In: O’Neill JA Jr, Grosfeld JL, Foukalsrud EW, Coran AG, Caldanone AA, editors. Principles of Pediatric Surgery. 2nd ed. St. Louis, MO: Mosby; 2003. pp. 385-394

[8] Goyal A, Jones MO, Couriel JM, Losty PD. Oesophageal atresia and tracheo-oesophageal fistula. Archives of Disease in Childhood. Fetal and Neonatal Edition. 2006;91:F381-F384

[9] Osei-Nketiah S, Hesse AA, AppeaduMensah W, Glover-Addy H, Etwire VK, Sarpong P. Management of oesophageal atresia in a developing country: Is primary repair forbidden? African Journal of Paediatric Surgery. 2016;13:114-119

[10] Karakus SC, Ozokutan BH, Bakal U, Ceylan H, Sarac M, Kul S, et al.
Delayed diagnosis: An important prognostic factor for oesophageal atresia in developing countries. Journal of Paediatrics and Child Health. 2016;52(12):1090-1094

[11] Ameh EA, Dogo PM, Nmadu PT. Emergency neonatal surgery in a developing country. Pediatric Surgery International. 2001;17:448-451

[12] Skandalakis JE, Ellis H. Embryologic and anatomic basis of esophageal surgery. The Surgical Clinics of North America. 2000;80(1):85

[13] Kluth D, Fiegel H. The embryology of the forgut. Seminars in Pediatric Surgery. 2003;12(1):3-9

[14] Beasley P. Anatomy of the pharynx and esophagus. In: Kerr AG, Gleeson M, editors. Scott-Brown's Otolaryngology. 6th ed. Oxford, UK: ButterworthHeinemann; 1997

[15] Postma GN, Seybt MW, Rees CJ. Esophagology. In: Snow JB, Wackym PA, editors. Ballinger's Otolaryngology Head \& Neck Surgery. 17th ed. Shelton, Conn: BC Decker Inc; 2009. pp. 975-995

[16] Swigart LL. The esophageal arteries. Surgery, Gynecology \& Obstetrics. 1950;90:234-243

[17] Geboes K, Geboes KP, Maleux G. Vascular anatomy of the gastrointestinal tract. Best Practice \& Research. Clinical Gastroenterology. 2001;15(1):1-14

[18] Potter SE. Observations on the intrinsic blood supply of the esophagus. Archives of Surgery. 1950;61:944-948

[19] Shapiro AL. The esophageal arteries: Their configurational anatomy and variations in relation to surgery. Annals of Surgery. 1950;131:171-185

[20] Butler H. The veins of the oesophagus. Thorax. 1951;6:276-296 
[21] Sasaki CT, Kim YH, Sims HS, et al. Motor innervation of the human cricopharyngeus muscle. Annals of Otology, Rhinology, and Laryngology. 1999;108(12):1132-1139

[22] Goyal RK, Martin SB, Shapiro J, et al. The role of cricopharyngeus muscle in pharyngoesophageal disorders. Dysphagia. 1993;8(3):252-258

[23] Harmon CM, Coran AG. Congenital anomalies of the esophagus. In: Coran AG, Adzick NS, Krummel TM, et al., editors. Pediatric Surgery. 7th ed. Vol. 1. Philadelphia, USA: Elsevier Saunders; p. 896 (chapter 69)

[24] Beasley SW. Esophageal atresia and tracheoesophageal fistula. In: Oldham KT, Colombani PM, Foglia RP, Skinner MA, editors. Principles and Practice of Pediatric Surgery. 4th ed. Vol. 2. Philadelphia, USA: Lippincott Williams \& Wilkins; p. 1040 (chapter 65)

[25] Harmon CM, Coran AG. Congenital anomalies of the esophagus. In: Coran AG, Adzick NS, Krummel TM, et al., editors. Pediatric Surgery. 7th ed. Vol. 1. Philadelphia, USA: Elsevier Saunders; p. 897 (chapter 69)

[26] Brosens E, Marsch F, de Jong EM, et al. Copy number variations in 375 patients with oesophageal atresia and/or tracheoesophageal fistula. European Journal of Human Genetics. 2016;24(12):1715-1723

[27] Al-Salem AH, Tayeb M, Khogair S, et al. Esophageal atresia with or without tracheoesophageal fistula: Success and failure in 94 cases. Annals of Saudi Medicine. 2006;26(2):116-119

[28] Shulman A, Mazkereth R, Zalel Y, et al. Prenatal identification of oesophageal atresia: The role of ultrasonography for evaluation of functional anatomy. Prenatal Diagnosis. 2002;22(8):669-674
[29] Tröbs RB, Nissen M, Wald J. The upper pouch in oesophageal atresia shows proportional growth during late foetal life. Acta Paediatrica. 2018;107(9):1648-1649

[30] Garabedian C, Sfeir R, Langlois C, Bonnard A, Khen-Dunlop N, Gelas T, et al. Does prenatal diagnosis modify neonatal treatment and early outcome of children with esophageal atresia? American Journal of Obstetrics and Gynecology. 2015;212:e1-e7

[31] Bradshaw CJ, Thakkar H, Knutzen L, Marsh R, Pacilli M, Impey L, et al. Accuracy of prenatal detection of tracheoesophageal fistula and oesophageal atresia. Journal of Pediatric Surgery. 2016;51(8):1268-1272

[32] Summerour V, Stevens PS, Lander AD, Singh M, Soccorso G, Arul GS. Characterization of the upper pouch tracheo-oesophageal fistula in oesophageal atresia. Journal of Pediatric Surgery. 2017;52(2):231-234

[33] Randolf JG, Newman KD, Anderson KD. Current results in repair of esophageal atresia with tracheaoesophageal fistula using physiologic status as a guide to therapy. Annals of Surgery. 1989;209:526, discussion 530

[34] Poenaru D, Laberge JM, Neilson IR, et al. A new prognostic classification for esophageal atresia. Surgery. 1993;113:426

[35] Spitz L, Kiely EM, Morecroft JA, Drake DP. Esophageal atresia: At-risk groups for the 1990s. Journal of Pediatric Surgery. 1994;29:723

[36] Driver CP, Shanker KR, Jones MO, et al. Phenotypic presentation and outcome of esophageal atresia in the era of Spitz classification. Journal of Pediatric Surgery. 2001;36(9):1419-1421

[37] Konkin DE, O’Hali AW, Webber EM, Blair GK. Outcomes in esophageal 
atresia and tracheoesophageal fistula. Journal of Pediatric Surgery. 2003;38(12):1726-1729

[38] Yagyu M, Gitter H, Richter B, et al. Esophageal atresia in Bremen, Germany-Evaluation of preoperative risk classification in esophageal atresia. Journal of Pediatric Surgery. 2000;35(4):584-587

[39] Barnhart DC, Area MJ, Coran AG. Esophageal atresia with or without tracheoesophageal fistula. In: Glick PL, Pearl RH, Irish MS, Caty MG, editors. Pediatric Surgery Secrets. Philadelphia, USA: Hanley \& Belfus Inc.; 2001. p. 35

[40] Prakash KR, Bhardwaj N, Dwivedi D, et al. Thoracoscopic repair of esophageal atresia with tracheoesophageal fistula: Basics of technique and its nuances. Journal of Indian Association of Pediatric Surgeons. 2016;21(3):120-124

[41] Okuyama H, Koga H, Ishimaru T, et al. Current practice and outcomes of thoracoscopic esophageal atresia and tracheoesophageal fistula repair: A multi-institutional analysis in Japan. Journal of Laparoendoscopic \& Advanced Surgical Techniques. Part A. 2015;25(5):441-444

[42] Lee S, Lee SK, Seo JM.

Thoracoscopic repair of esophageal atresia with tracheoesophageal fistula: Overcoming the learning curve. Journal of Pediatric Surgery. 2014;49(11):1570-1572

[43] Rothenberg S. Thoracoscopic repair of esophageal atresia and tracheo-esophageal fistula in neonates: The current state of the art. Pediatric Surgery International. 2014;30(10):979-985

[44] Rothenberg SS, Flake AW. Experience with thoracoscopic repair of long gap esophageal atresia in neonates. Journal of Laparoendoscopic
\& Advanced Surgical Techniques. Part A. 2015;25(11):932-935

[45] Tainaka T, Uchida H, Tanano A, et al. Two-stage thoracoscopic repair of long-gap esophageal atresia using internal traction is safe and feasible. Journal of Laparoendoscopic \& Advanced Surgical Techniques. Part A. 2017;27(1):71-75

[46] van der Zee DC, Tytgat SHA, van Herwaarden MYA. Esophageal atresia and tracheo-esophageal fistula. Seminars in Pediatric Surgery. 2017;26(2):67-71

[47] Holcomb GW 3rd. Thoracoscopic surgery for esophageal atresia. Pediatric Surgery International. 2017;33(4):475-481

[48] García LI, Olivos PM, Santos MM, Guelfand CHM. Thoracoscopic repair of esophageal atresia with and without tracheoesophageal fistula. Revista Chilena de Pediatría. 2014;85(4):443-447

[49] Woo S, Lau S, Yoo E, Shaul D, Sydorak R. Thoracoscopic versus open repair of tracheoesophageal fistulas and rates of vocal cord paresis. Journal of Pediatric Surgery. 2015;50(12):2016-2018

[50] Shieh HF, Jennings RW. Long-gap esophageal atresia. Seminars in Pediatric Surgery. 2017;26(2):72-77

[51] van der Zee DC, Gallo G, Tytgat SH. Thoracoscopic traction technique in long gap esophageal atresia: Entering a new era. Surgical Endoscopy. 2015;29(11):3324-3330

[52] Sroka M, Wachowiak R, Losin M, et al. The Foker Technique (FT) and Kimura Advancement (KA) for the treatment of children with long-gap esophageal atresia (LGEA): Lessons learned at two European centers. European Journal of Pediatric Surgery. 2013;23:3-7 
[53] Bobanga ID, Barksdale EM. Foker technique for the management of pure esophageal atresia: Long-term outcomes at a single institution. European Journal of Pediatric Surgery. 2016;26(2):215-218

[54] Miyano G, Okuyama H, Koga H, et al. Type-A long-gap esophageal atresia treated by thoracoscopic esophagoesophagostomy after sequential extrathoracic esophageal elongation (Kimura's technique). Pediatric Surgery International. 2014;29(11):1171-1175

[55] Bogusz B, Patkowski D, Gerus S, et al. Staged thoracoscopic repair of long-gap esophageal atresia without temporary gastrostomy. Journal of Laparoendoscopic \& Advanced Surgical Techniques. Part A. 2018;28(12):1510-1512

[56] Sharma AK, Mangal D. Simple technique of bridging wide gap in esophageal atresia with tracheoesophageal fistula_- "Surgical innovation". Journal of Indian Association of Pediatric Surgeons. 2017;22(3):187-188

[57] Krishnan U, Faure C. Editorial: Oesophageal atresia-tracheoesophageal fistula. Frontiers in Pediatrics. 2017;5:190

[58] Sadreameli SC, McGrath-Morrow SA. Respiratory care of infants and children with congenital tracheooesophageal fistula and oesophageal atresia. Paediatric Respiratory Reviews. 2016;17:16-23

[59] Duvoisin G, Krishnan U. Gastric function in children with oesophageal atresia and tracheoesophageal fistula. Frontiers in Pediatrics. 2017;5:76

[60] Thakkar HS, Cooney J, Kumar N, Kiely E. Measured gap length and outcomes in oesophageal atresia. Journal of Pediatric Surgery. 2014;49(9):1343-1346
[61] Harmon CM, Coran AG. Congenital anomalies of the esophagus. In: Coran AG, Adzick NS, Krummel TM, et al., editors. Pediatric Surgery. 7th ed. Vol. 1. Philadelphia, USA: Elsevier Saunders; p. 911 (chapter 69)

[62] Manning PB, Morgan RA, Coran AG, et al. Fifty years experience with esophageal atresia and tracheoesophageal fistula. Beginning with Cameron Haight's first operation in 1935. Annals of Surgery. 1986;204:446

[63] Velligas-Alvarez F, Olvera-Duran J, Rodriguez-Aranda E, et al. Esophageal anastomotic failure: An experimental study. Archives of Medical Research. 2003;34:171

[64] Yanchar NL, Gordon R, Cooper M, et al. Significance of the clinical course of and early upper gastrointestinal studies in predicting complications associated with repair of esophageal atresia. Journal of Pediatric Surgery. 2001;36(5):815-822

[65] Svoboda E, Fruithof J, WidenmannGrolig A, et al. A patient led, international study of long term outcomes of esophageal atresia: EAT 1. Journal of Pediatric Surgery. 2018;53(4):610-615

[66] Arnaud AP, Rex D, Elliott MJ, et al. Early experience of thoracoscopic aortopexy for severe tracheomalacia in infants after esophageal atresia and tracheo-esophageal fistula repair. Journal of Laparoendoscopic \& Advanced Surgical Techniques. Part A. 2014;24(7):508-512

[67] Soccorso G, Parikh D. Esophageal replacement in children: Challenges and long-term outcomes. Journal of Indian Association of Pediatric Surgeons. 2016;21(3):98-105 



\title{
Chapter 4
}

\section{Meconium Ileus}

\author{
Udefiagbon Omogiade
}

\begin{abstract}
Meconium ileus is a type of neonatal intestinal obstruction that occurs when abnormally thick meconium impacts in the ileum causing blockage of intestinal flow. Most infants with meconium ileus have cystic fibrosis, a congenital condition characterized by abnormally thick intestinal secretions and pancreatic insufficiency. The pathogenesis of meconium ileus is due to hyperviscous mucus secreted by abnormal intestinal glands, abnormal concentrating processes in the proximal small intestine, and pancreatic enzyme insufficiency. The clinical presentation of meconium ileus is that of abdominal distention, bilious vomiting, and failure to pass meconium. Cases of meconium ileus are usually evaluated with plain abdominal radiograph and contrast enema. Numerous air-filled loops of bowel on the supine view with characteristic absence of air-fluid levels are commonly seen on the radiograph, but the presence of calcification suggests intestinal perforation. Contrast enema examination is useful in cases with microcolon. Uncomplicated meconium ileus obstruction can be relieved by giving one or more dilute diatrizoate sodium enema (with Nacetylcysteine added) under fluoroscopy. Surgery is indicated when there is progressive distention or signs of clinical deterioration despite multiple enemas, as well as in complicated cases like meconium peritonitis, ileal atresia or stenosis, ileal perforation, and volvulus with or without pseudocyst formation.
\end{abstract}

Keywords: meconium ileus, cystic fibrosis, ileal obstruction, gastrografin enema, nonoperative treatment, enterotomy, enterostomy, resection and anastomosis

\section{Introduction}

Meconium ileus is a type of neonatal intestinal obstruction that occurs when abnormally thick and tenacious meconium becomes impacted, thus creating a blockage in a part of the distal small intestine, usually the ileum [1,2]. It accounts for about 30-33\% of cases of neonatal small intestinal obstruction [3]. Meconium ileus is a rare condition affecting only 1 in 25,000 babies [4]. It occurs in either a simple or a complicated form and is said to be the earliest clinical manifestation of cystic fibrosis occurring in approximately $16-20 \%$ of patients with cystic fibrosis [4]. Cystic fibrosis is a disease condition characterized by abnormally thick intestinal secretions and pancreatic insufficiency.

While majority of patients with meconium ileus have the disease cystic fibrosis (80-90\%), a few of them do not have it; approximately $20 \%$ of one series of cases of meconium ileus did not have cystic fibrosis [5]. Preterm infants whose mothers had medications to slow down labor are sometimes associated with meconium ileus. 
Meconium is the first series of stools that a newborn pass. It is formed during intrauterine life and consists of shed intestinal epithelial cells, bile, succus entericus, mucus, lanugo, and amniotic fluid ingested in utero. It is a dark olive green viscous and almost odorless substance that comprises the initial stools of the newborn. Meconium contains lactic acid-producing bacteria (e.g., Lactobacillus) and the so-called enteric bacteria family (e.g., Escherichia coli) [6]. It is usually evacuated within the first 24-48 hours after birth following which the usual yellowish feces are passed by the neonate. However, there might be in utero evacuation of meconium as a result of a vagal response due to perinatal stress to the fetus. Such newborn immediately after delivery may develop signs of respiratory distress from meconium aspiration syndrome.

Meconium ileus may be associated with complications such as meconium peritonitis, ileal atresia or stenosis, ileal perforation, and volvulus with or without pseudocyst formation [7-13]. The infants with cystic fibrosis are more likely to present with complicated meconium ileus [14].

\section{Detailed overview of meconium ileus}

An overview of meconium ileus in terms of etiology, pathophysiology, clinical features, investigations, and treatment will now be undertaken.

\subsection{Etiology}

Up to $20 \%$ of babies with cystic fibrosis are born with meconium ileus, and almost all babies with meconium ileus have cystic fibrosis $[15,16]$. Cystic fibrosis is caused by gene mutations in the cystic fibrosis transmembrane conductance regulator (CFTR) encoding gene [17-19]. The loss of CFTR-mediated Cl-and/or HCO3- transport by the intestinal epithelium and/or from pancreatic dysfunction is postulated as the pathogenesis of meconium ileus [20-24]. Cystic fibrosis is characterized by the triad of chronic obstruction and infection of the respiratory tract, exocrine pancreatic insufficiency, and elevated sweat chloride levels.

The pathogenesis of meconium ileus is due to hyperviscous mucus secreted by abnormal intestinal glands, abnormal concentrating processes in the proximal small intestine, and pancreatic enzyme insufficiency.

The histology is characterized by the presence of distended goblet cells in the intestinal mucosa.

\subsection{Pathophysiology}

The simple form of meconium ileus is characterized by thickened sticky meconium obstructing the ileum with consequent proximal dilatation, bowel wall thickening, and congestion. Immediately beyond the level of the obstructing inspissated meconium in the terminal ileum, there may be a few separate gray-white globular meconium pellets. Further distally, the colon is narrow and empty-the microcolon.

The complicated form may result in volvulus, atresia, necrosis, perforation, meconium peritonitis, and pseudocyst formation. These complications may manifest as incidental findings on abdominal radiographs or with clinical features suggestive of bowel obstruction caused by reactive fibro-adhesive bands due to the meconium in the peritoneal cavity or as clinical features of peritonitis. If a neonate at birth manifests features of peritonitis, it is likely due to meconium peritonitis 
secondary to meconium ileus bowel perforation. This may also result in intestinal atresia, intraperitoneal calcifications, or ascites [25]. Meconium pseudocyst is formed when the extruded meconium becomes walled off; it is a cystic mass with rim calcification [26].

In utero, about $50 \%$ of meconium ileus cases may be complicated by intestinal perforation, meconium peritonitis, volvulus, and ischemic necrosis of the bowel that results in stenosis or atresia [4].

Meconium ileus patients are at risk for developing cholestasis, especially if they are on total parenteral nutrition. As such, alkaline phosphatase (ALP), alanine aminotransferase (ALT), aspartate aminotransferase (AST), and bilirubin levels should be monitored weekly in such infants.

\subsection{Clinical features}

At birth, the neonate may be apparently normal. However, with progression of time and feeding, the infant develops abdominal distention, bilious vomiting, and failure to pass meconium. Sometimes thickened distended bowel loops are observed through the abdominal wall filled with rubbery meconium which when palpated feel characteristically doughy [3]. Bowel sounds tend to be hypoactive, and digital rectal examination may be followed by passage of pale mucosal plugs. Meconium pellets might be palpated in the scrotum of some infants who had in utero bowel perforation. In cases complicated by peritonitis or when postnatal perforation has occurred, the infant presents with respiratory distress, marked abdominal distention with abdominal erythema, significant abdominal tenderness, and ascites.

\subsection{Investigations}

Cases of meconium ileus are usually evaluated with abdominal radiograph in which meconium might have a mottled appearance or be invisible [27].

Plain abdominal radiographs are routinely the first imaging done for cases of meconium ileus. They show numerous air-filled loops of bowel on the supine view with characteristic absence of air-fluid levels on the upright view due to the tenacious meconium and the abnormal mucous-gland secretion [5]. Although the absence of air-fluid levels strongly suggests meconium ileus, the presence of airfluid levels does not exclude it as it may occasionally be demonstrated in some cases. In some cases of meconium ileus, the admixture of meconium and bowel gas gives a soap-bubble appearance usually in the right lower quadrant (Neuhauser sign). The presence of calcification, free air, or multiple air-fluid levels suggests intestinal perforation [4].

A contrast enema examination is useful in confirming the diagnosis of meconium ileus in which microcolon is seen; this differentiates it from meconium plug syndrome in which a normal or dilated colon is seen [25]. The microcolon, which represents the underused colon, could also be seen in other congenital conditions causing complete intrauterine obstruction of the distal small bowel such as ileal atresia; however for cases of meconium ileus, the presence of meconium pellets distending the distal ileum is usually identified when the contrast refluxes into the small bowel, and the diagnosis is confirmed (Figure 1).

Water-soluble agents are typically used in contrast evaluation of meconium ileus, and several of such contrast agents have been used. The hyperosmolar meglumine (GastrografinRx) diluted at ratio 1:3 to water used to be the mainstay, but some radiologists have stopped using it because of the occurrence of deaths 


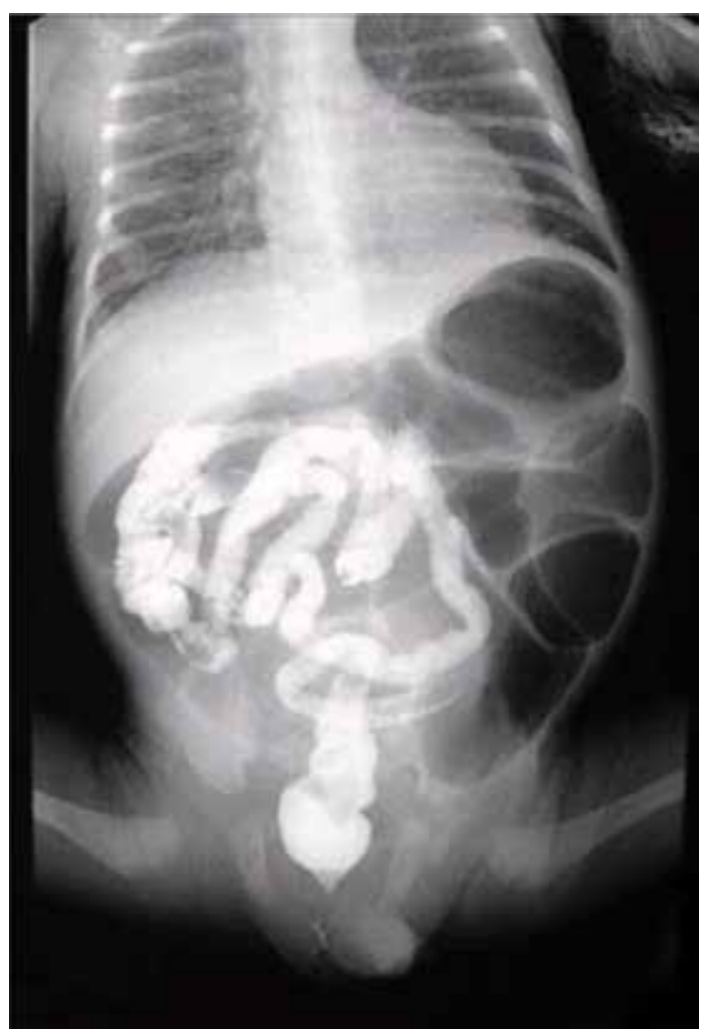

Figure 1.

Contrast enema showing microcolon and meconium pellets in the terminal ileum [courtesy Radiopaedia].

from fulminant colitis and dehydration sometimes reported with its use [28]. Also, the report of the Cystic Fibrosis Foundation Consensus Conference on gastrointestinal disorders concluded that there is no scientific evidence that hyperosmolar Gastrografin enema is any better than an iso-osmolar or hypo-osmolar enema. Nevertheless, many radiologists use it safely by ensuring appropriate dilution ratios. Adequate monitoring of fluid and electrolyte balance before, during, and after the contrast study is essential to avert potential fluid shifts with consequent hypovolemia which is worsened when bowel perforation and contrast leak occur. Nonionic contrast agents like Hypaque and Omnipaque are becoming popular with many radiologists since they have less risk of dehydration or colitis. Because of the tenacious and sticky nature of meconium, mucolytic agents like acetylcysteine are sometimes mixed with the contrast enema solution to aid passage of the meconium.

Meconium peritonitis may be an incidental abdominal radiograph finding in which the extruded meconium may be calcified or the radiograph may only suggest fluid in the abdomen when no calcification is present. When the calcification appears amorphous and curvilinear suggesting cystic loculation of the peritoneum, the term cystic or pseudocystic meconium peritonitis is used [5].

Prenatal ultrasound scan done at 17-18 weeks gestational age may show signs suggestive of meconium ileus; this include enlarged bowel loops or a mass with proximal bowel distention (likely cystic meconium peritonitis) [11, 12]. Also, calcified meconium may be seen if meconium peritonitis has already occurred. Also there might be polyhydramnios. 
Postnatal ultrasound scan is seldom necessary for meconium peritonitis, as the findings on plain radiographs are usually diagnostic. However, ultrasonography may be useful when cystic masses are present. The cystic masses often appear circumscribed and heterogeneous with sonolucent areas seen within the cyst suggestive of fluid. They demonstrate increased echogenicity resulting from debris and calcifications, and loops of fluid-filled bowel bound to the matrix of the associated adhesions may be noted. The cyst wall may be thick or thin. Multiple speckled echoes are seen with free-floating meconium in the abdomen, and these result in the snowstorm configuration.

Zangheri et al. created the following classification system related to perinatal outcome [29]:

- Grade 0: isolated intra-abdominal calcifications (IAC)

- Grade I: IAC and one of the following: ascites, pseudocyst, or bowel dilatation

- Grade II: IAC and two of the following: ascites, pseudocyst, or bowel dilatation

- Grade III: all of the above (IAC, ascites, pseudocyst, and bowel dilatation)

Patients diagnosed with meconium ileus should be tested for cystic fibrosis; the sweat chloride test should be done [25].

\subsection{Treatment}

\subsubsection{Initial medical management}

Meconium ileus cases, both simple and complicated, are approached as intestinal obstruction and as such would require urgent initial resuscitative measures. These include intravenous fluid resuscitation, nasogastric decompression, urethral catheterization for hourly urinary monitoring, multiparameter vital sign monitoring, intravenous antibiotic therapy, laboratory evaluation of full blood count, coagulation work-up, and serum electrolytes, urea, and creatinine with necessary corrections instituted. Where necessary, mechanical respiratory support is provided. Once the infant has been optimized, the decision for nonoperative or operative management is taken based on the presentation.

\subsubsection{Nonoperative treatment}

Nonoperative management can be achieved by diatrizoate meglumine enemas as first described by Noblett in 1969 [30]. Variations on his approach have been established as effective first-line treatment for uncomplicated meconium ileus.

Uncomplicated meconium ileus obstruction can be relieved by giving one or more dilute diatrizoate sodium or diatrizoate (gastrografin) enema (with $\mathrm{N}$-acetylcysteine added) under fluoroscopic guidance. The hyperosmolar nature of this compound increases the influx of fluid into the bowel lumen to liquefy the viscid meconium and thus facilitate its expulsion with consequent large gastrointestinal water losses. While carrying out this procedure, therefore, adequate intravenous fluid administration must be ensured to prevent hypovolemia. 


\subsubsection{Treatment algorithm}

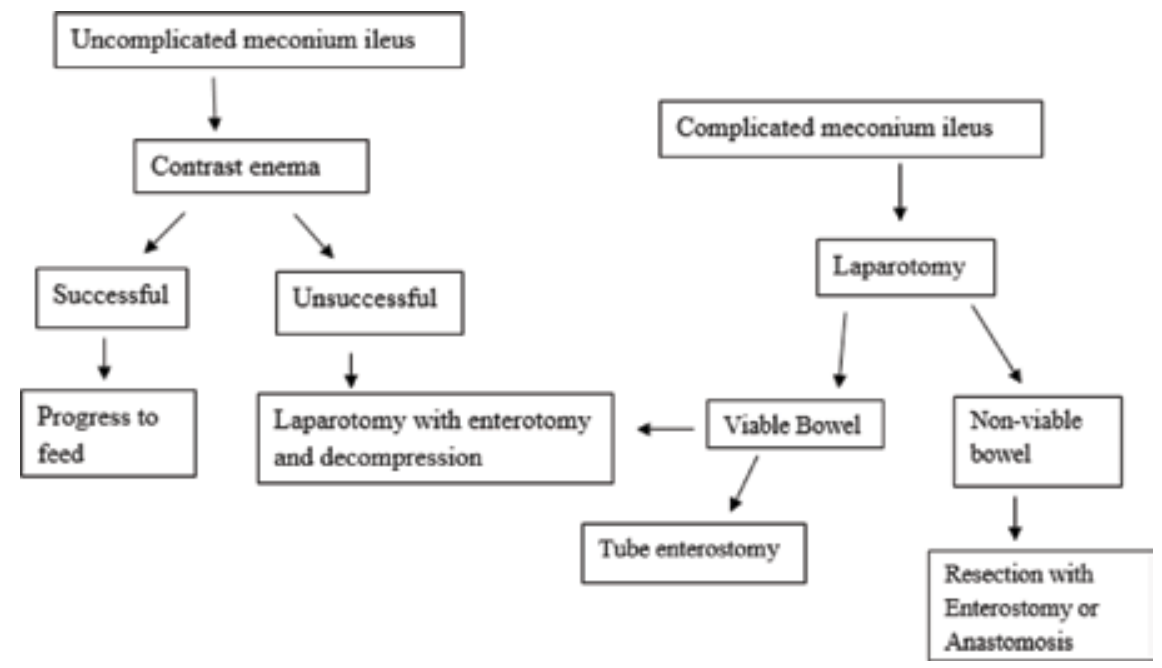

Diatrizoate meglumine (GastrografinRx) is a hyperosmolar, water-soluble, radiopaque solution containing $0.1 \%$ polysorbate 80 (Tween 80 ) and $37 \%$ organically bound iodine with osmolarity of $1900 \mathrm{mOsm} / \mathrm{L}$. Success rate of $63-83 \%$ have been reported for gastrografin enemas for patients with uncomplicated meconium ileus [31].

Noblett's criteria for nonoperative gastrografin enema therapy [30]:

- Other causes of neonatal distal intestinal obstruction must first be excluded.

- There should be no clinical or radiologic signs of complications like volvulus, gangrene, perforation, peritonitis, and atresia of the small bowel.

- Ensure adequate fluid and electrolyte replacement and correction of hypothermia as preparatory measures before the enema.

- Provision for adequate resuscitation and hydration in anticipation of transient osmotic fluid losses associated with the hyperosmolar enema.

- The enema must be carried out under fluoroscopic guidance.

- Intravenous antibiotics should be administered to the infant.

- Assurance of close surgical supervision from the initial evaluation through the hospital course.

To carry out the enema, a two-way Foley's catheter is inserted into the rectum through which a $25-50 \%$ solution of gastrografin is slowly infused at low hydrostatic pressure under fluoroscopic control. The balloon of the catheter should not be inflated to minimize the risk of rectal perforation. Upon instillation, fluid is drawn into the intestinal lumen by osmosis, and this hydrates and softens the meconium mass. For very inspissated meconium, $1 \% \mathrm{~N}$-acetylcysteine may be added to the enema solution for better deconcentration. The procedure is usually followed by rapid passage of loose meconium (liquefied to some extent), and this continues for the next 24-48 hours. 
Although the perforation that occurs during enema administration can usually be seen on fluoroscopy, it is important to obtain an immediate abdominal radiograph after completion of the gastrografin enema to rule out bowel perforation and a late abdominal radiograph (8-12 hours later or as clinically indicated) to confirm evacuation of the obstruction and to exclude late perforation [31].

Sometimes a second gastrografin enema or serial gastrografin enemas can be performed at 6-24 hour intervals if evacuation is incomplete or if the first attempt at gastrografin evacuation does not reflux contrast into dilated bowel. Administration of a $10 \% \mathrm{~N}$-acetylcysteine solution ( $5 \mathrm{~mL}$ q6h) through a nasogastric tube to liquefy upper gastrointestinal secretions as suggested by Noblett is also useful in such cases [30]. The potential complications associated with the gastrografin enema procedure include perforation, hypovolemic shock, and ischemia.

The risk of perforation during the procedure increases with repeated enemas. Late perforations, usually occurring 12-48 hours after the enema, may be due to direct injury to the bowel mucosa by the contrast medium, severe bowel distention by fluid osmotically drawn into the intestine, or extensive bowel necrosis.

Nonoperative treatment can be done for infants with peritoneal (or scrotal) calcifications on radiography who are presumed to have had meconium peritonitis in utero but who show no signs of obstruction and are passing meconium without difficulty [32].

In nonoperative management, if the enema was successful and the features of bowel obstruction have resolved, usually within 48 hours, the infant is commenced on feeds with pancreatic enzyme supplements added for infants with confirmed cystic fibrosis.

\subsubsection{Surgical treatment}

In uncomplicated meconium ileus, surgical exploration is indicated when there is progressive distention or signs of clinical deterioration or peritonitis despite multiple enemas. Whereas in complicated cases (e.g., meconium peritonitis, ileal atresia or stenosis, ileal perforation, and volvulus with or without pseudocyst formation), surgery is always indicated.

Indications for surgical management in meconium ileus [31]:

- Persistent or worsening abdominal distension

- Persistent bowel obstruction

- Enlarging abdominal mass

- Intestinal atresia

- Volvulus

- Perforation

- Meconium cyst formation with peritonitis

- Bowel necrosis

- Conditions associated with cystic fibrosis and meconium Ileus 
In the operative management of simple uncomplicated meconium ileus, the aim is to evacuate meconium from the intestine without resecting any bowel segment; however, this might be inevitable in certain instances. On the other hand, complicated meconium ileus requires resection more often and may necessitate the use of temporary stomas.

The fibrous wall of the pseudocyst is debrided without sacrificing viable intestine. Extensive adhesiolysis is required for adhesive obstruction due to meconium peritonitis; these adhesions are typically dense and very vascular. It is not necessary to perform a radical debridement of all meconium calcified plaque encountered, as long as the obstruction is relieved [32].

The surgical approach for treatment of uncomplicated meconium ileus should be individualized for each infant, although many procedures have been proposed over the years with variable success rates achieved. In all cases, uncomplicated or complicated, the following procedures are commonly done:

- Enterotomy and decompression

- Enterostomy (with or without tube) with subsequent irrigation

- Resection and enterostomy

- Resection and anastomosis

\subsubsection{Enterotomy and decompression}

An enterotomy is made on the antimesenteric border of the dilated ileum for instillation of irrigation solution (dilute acetylcysteine or saline solution) which help to loosen the inspissated meconium and liquefy it for effective evacuation through the enterotomy. The irrigation solution is introduced using a size 10 French catheter, and both the proximal and distal loops of bowel are irrigated. After complete decompression of inspissated meconium, the enterotomy is closed transversely. An appendectomy is performed with the specimen sent for histologic examination to detect the presence of ganglion cells, as well as possible presence of mucous plugging of the crypts and exuberant intraluminal mucinous material, which are suggestive of cystic fibrosis. Postoperatively, a gentle anal dilatation and rectal irrigation may sometimes be required for further evacuation of large amount of meconium passed distally into the colon during intraoperative irrigation. Enterotomy and decompression are usually indicated for simple uncomplicated meconium ileus. A supraumbilical transverse incision or transverse right lower abdominal incision can be used for the procedure.

\subsubsection{Enterostomy with subsequent irrigation}

In cases where the irrigation done after an enterotomy cannot effectively evacuate the inspissated meconium despite a patient approach, an indwelling ostomy tube (e.g., T-tube) can be inserted for postoperative bowel irrigation decompression. The irrigations are started on the first postoperative day and continued for 7-14 days. After successfully evacuating the inspissated meconium, the tube is removed, and the enterocutaneous fistula thus formed is allowed to close spontaneously. T-tube enterostomy was first described by Harberg et al. in 1981 [33]. Enterostomy for postoperative irrigation can also be done without using tube; in which case the ileal opening is sutured to skin and the bowel tacked to the fascia in standard fashion. 


\subsubsection{Resection with enterostomy or anastomosis}

Bowel resection is indicated when meconium ileus is associated with a nonviable bowel, bowel perforation, atresia, volvulus, and the like. Resection is usually combined with enterostomy procedure, but primary anastomosis may be done if the intraoperative findings and the patient's general condition are favorable. The disadvantages of the procedures involving resection and stoma(s) or anastomosis are potential postoperative fluid losses through high-volume stomas, bowel shortening by resection, and the need for a second procedure to reestablish intestinal continuity $[25,31]$. Hence, they are rarely used today.

Various stoma operations have been described with the most widespread being the Bishop-Koop-type anastomosis. This is a Roux-en-Y construct in which the distal limb is brought out as an end stoma and the proximal bowel is anastomosed end-toside approximately $4 \mathrm{~cm}$ from the opening of the distal segment (Figure 2). Normal gastrointestinal transit is permitted by this technique, and should distal obstruction occur, it provides a means for management through the ileostomy [25, 31].

The reverse of the Bishop-Koop enterostomy is the proximal enterostomy, described by Santulli and Blanc in 1961 [25, 31]. In this technique, the end of the distal limb is anastomosed to the side of the proximal limb after resection, while the end of the proximal limb is brought out as the enterostomy (Figure 2). This arrangement enhances proximal irrigation and decompression, thus making intraoperative evacuation of the dilated proximal bowel loop unnecessary. A catheter can be inserted into the distal limb through the stoma for irrigation of the distal bowel. The proximal stoma created in this technique predisposes to high-output losses with inherent risk of dehydration.

The Mikulicz enterostomy, first reported by Gross in 1953, consists of a doublebarrel stoma in which the two ends are sutured together side to side for some length

a

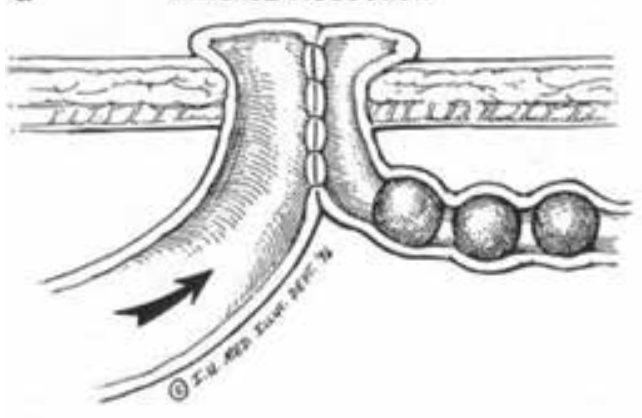

C

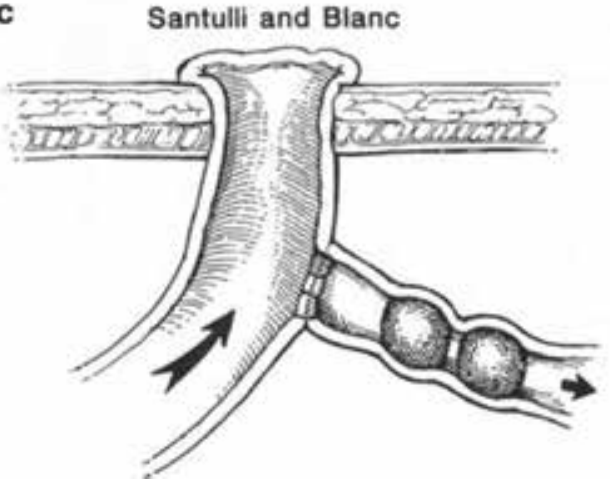

b Bishop-Koop Resection

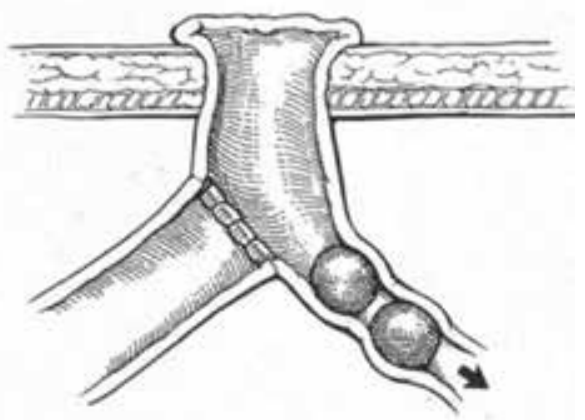

d

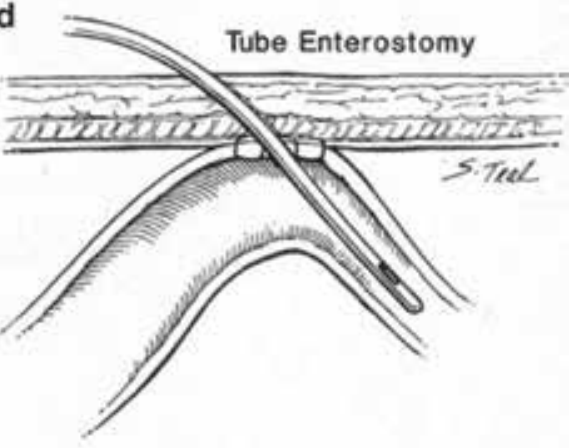

Figure 2.

Schematic description of some enterostomies as copied from Ref. [36]. 
proximal to the end of the stoma (Figure 2) [25, 31]. It was designed for bedside stoma closure in which the common wall was crushed and obliterated with a specially designed clamp and the bowel ends were closed over the top. It has the following distinct advantages:

- The procedures reduce operating and anesthetic times because complete evacuation of inspissated meconium is unnecessary.

- The procedures avoid intra-abdominal anastomosis, which eliminates the risk of anastomotic leakage.

- The bowel can be opened after complete closure of the abdominal wound; this reduces the risk of intraperitoneal contamination.

Swenson was the first to suggest resection with primary anastomosis in 1962 $[25,31]$. Anastomotic leakage was initially a major issue with such operation; however some authors have reported improved results with adequate resection of the compromised bowel, complete evacuation of proximal and distal meconium, and preserving an adequate blood supply to the anastomosis [34, 35].

\subsection{Postoperative care}

Immediately postoperatively, management involves ongoing resuscitation with special attention given to replacement of the fluid losses caused by surgery and preoperative hyperosmolar enemas (if attempted), as well as correction of ongoing losses (i.e., losses from nasogastric suction and ileostomy) [31]. Also, the infant is initially on bowel rest with general supportive care provided after any major laparotomy. The oral gastric tube is maintained until bowel function returns, and further acetylcysteine irrigations can be done via the tube as described for nonoperative management. Combining this with rectal irrigations may further aid passage of retained meconium in the distal loop as well. Most infants will need central venous access for parenteral nutrition during this period. If cystic fibrosis has not been confirmed preoperatively, the sweat chloride test should be done to confirm or rule it out. Close attention has to be given to pulmonary care in infants with cystic fibrosis. Multiple pediatric subspecialists including gastroenterologists, geneticists, pulmonologists, and pediatric surgeons are required for a good outcome of management in infants with meconium ileus, more so when they have cystic fibrosis. Once they have established a normal stooling pattern (usually within 1-2 weeks postoperative), they are commenced on graded oral feedings with pancreatic enzyme supplementation. Infants with uncomplicated meconium ileus and cystic fibrosis may receive breast milk or routine infant formula, enzymes, and vitamins, while complicated cases would benefit from predigested infant formulas (e.g., Alimentum and Pregestimil), for enteral feeding [31]. For those with stomas, administering of ostomy-drip feeds of glutamine-enriched formula at low volumes enhances bowel growth and helps prevent bacterial translocation [31]. After 4-6 weeks, when symptoms would have resolved and the infant attained an adequate weight gain, the stomas can be taken down. It is advisable to perform a distal contrast study to rule out obstruction before embarking on this procedure of reanastomosis.

\subsection{Postoperative complications}

Short-term complications are uncommon in infants with simple meconium ileus. 
Infants who have had significant (i.e., > 33\%) bowel resection may develop short bowel syndrome, especially if the ileocecal valve has been resected.

Short bowel syndrome predisposes to acidic intestinal environment that inactivates pancreatic enzymes and prevents dissolution of enteric-coated microcapsules. As such, histamine 2 receptor blockers are useful adjunct to pancreatic enzyme therapy in patients with significant bowel resections.

There are excessive fluid and sodium losses in those with stomas.

Mucus plugging and atelectasis can occur postoperatively, hence the need to initiate vigorous prophylactic pulmonary care with chest physiotherapy.

High-dose pancreatic enzyme supplementation has been associated with the development of colonic strictures and distal intestinal obstruction syndrome.

Long-term complications are mostly common to patients with cystic fibrosis.

Some infants, especially those who had meconium peritonitis may present years later with bowel obstruction due to adhesions or segmental volvulus.

Some older patients have been known to develop bowel obstruction from inspissated stools in the ileum and colon; this condition is known as "meconium ileus equivalent" [32].

\section{Conclusion}

Meconium ileus is a cause of neonatal small intestinal obstruction which mainly affects the ileum and common in infants with cystic fibrosis. Contrast (gastrografin) enema is usually diagnostic and may sometimes be therapeutic. Infants who have unsuccessful management with enemas and those with complications related to the obstruction, including volvulus, perforation, or atresia, require operative intervention.

At laparotomy, a small enterotomy is done for those with simple meconium ileus to irrigate the bowel lumen with acetylcysteine solution and thus promote effective evacuation of the highly viscous meconium. Patients with complicated meconium ileus may require bowel resection with anastomosis or tube enterostomy or creation of a stoma. Various types of stomas have been described over the years for management of the disease, but the Bishop-Koop enterostomy seems to be widespread.

Most patients respond well to therapy in the short-term but need to be followed closely for long-term complications like bowel obstruction, which has many potential causes in these patients. Advances in perinatal diagnosis and management of meconium ileus and cystic fibrosis have vastly improved the outlook for affected infants.

\section{Conflict of interest}

There is no "conflict of interest."

\section{Notes/thanks/other declarations}

None. 


\section{Author details}

Udefiagbon Omogiade

Department of Pediatric Surgery, Irrua Specialist Teaching Hospital, Irrua, Nigeria

*Address all correspondence to: dokernie11@gmail.com

\section{IntechOpen}

(C) 2019 The Author(s). Licensee IntechOpen. This chapter is distributed under the terms of the Creative Commons Attribution License (http://creativecommons.org/licenses/ by/3.0), which permits unrestricted use, distribution, and reproduction in any medium, provided the original work is properly cited. (cc) BY 


\section{References}

[1] Carlyle BE, Borowitz DS, Glick PL. A review of pathophysiology and management of foetuses and neonates with meconium ileus for the pediatric surgeon. Journal of Pediatric Surgery. 2012;47:772-781

[2] Copeland DR, St Peter SD, Sharp S, et al. Diminishing role of contrast enema in simple meconium ileus. Journal of Pediatric Surgery. 2009;44:2130-2132

[3] Cochran WJ. Meconium ileus. Merck Manuals. Kenilworth, NJ, USA: Merck \& Co., Inc.; 2018

[4] Leung AKC, Wong AL, Kao P. Meconium ileus: Causes and complications. Modern Medicine Network Special Report; 2005

[5] Hekmatnia A, Mc Hugh K, Hiorns MP. Meconium Ileus Imaging. In: Lin EC, Chief Editor; 2019. Medscape (C). Web MD LLC

[6] Jimenez E et al. Is meconium from healthy newborns actually sterile? Research in Microbiology;159(3):187-193

[7] Burge D, Drewett. Meconium plug obstruction. Pediatric Surgery International. 2004;20(2):108-110

[8] Fakhoury K, Durie PR, Levison H. Meconium ileus in the absence of cystic fibrosis. Archives of Disease in Childhood. 1992;67(10 Spec):1204-1206

[9] Lang I, Daneman A, Cutz E, et al. Abdominal calcification in cystic fibrosis with meconium ileus: Radiologic-pathologic correlation. Pediatric Radiology. 1997;27(6):523-527

[10] Lim CT, Yip CH, Chang KW. Meconium ileus-A rare cause of neonatal intestinal obstruction in Malaysia. Singapore Medical Journal. 1994;35(1):74-76
[11] Boczar M, Sawicka E, Zybert K. Meconium ileus in newborns with cystic fibrosis-Results of treatment in the group of patients operated on in the years 2000-2014. Developmental Period Medicine. 2015;19(1):32-40

[12] Takacs ZF, Meier CM, Solomayer G. Prenatal diagnosis and management of an intestinal volvulus with meconium ileus and peritonitis. Archives of Gynecology and Obstetrics. 2014;290(2):385-387

[13] Jerdee T, Newman B, Rubesova E. Meconium in perinatal imaging: Associations and clinical significance. Seminars in Ultrasound, CT, and MR. 2015;36(2):161-177

[14] Burke MS, Ragi JM, Karamanoukian HL, et al. New strategies in the nonoperative management of meconium ileus. Journal of Pediatric Surgery. 2002;37:760-764

[15] van der Doef HP, Kokke FT, van der Ent CK, Houwen RH. Intestinal obstruction syndromes in cystic fibrosis: Meconium ileus, distal intestinal obstruction syndrome, and constipation. Current Gastroenterology Reports. 2011;13(3):265-270. DOI: 10.1007/s11894-011-0185-9

[16] Gelfond D, Borowitz D. Gastrointestinal complications of cystic fibrosis. Clinical Gastroenterology and Hepatology. 2013;11(4):333-342. DOI: 10.1016/j.cgh.2012.11.006

[17] Welsh MJ, Ramsey BW, Accurso F, Cutting GR. The metabolic and molecular basis of inherited disease. In: Scriver CR, Beaudet AL, Sly WS, Valle D, Childs B, Vogelstein B, editors. Cystic Fibrosis. 8th ed. New York, New York, USA: McGraw-Hill; 2001. pp. 5121-5189

[18] Rowe SM, Miller S, Sorscher EJ. Cystic fibrosis. The New England Journal 
of Medicine. 2005;352(19):

1992-2001. DOI: 10.1056/NEJMra043184

[19] Quinton P. Physiological basis of cystic fibrosis: A historical perspective. Physiological Reviews. 1999;79(suppl 1): S3-S22

[20] Clarke LL, Harline MC. Dual role of CFTR in cAMP-stimulated HCO3secretion across murine duodenum. American Journal of Physiology. Cell Physiology. 1998;274(4 pt 1):G718-G726

[21] Clarke LL, Stien X, Walker NM. Intestinal bicarbonate secretion in cystic fibrosis mice. Journal of the Pancreas: JOP. 2001;2(4 suppl):263-267

[22] Harmon GS et al. Pharmacological correction of a defect in PPAR-gamma signaling ameliorates disease severity in Cftr-deficient mice. Nature Medicine. 2010;16(3):313-318. DOI: 10.1038/ $\mathrm{nm} .2101$

[23] Garcia MA, Yang N, Quinton PM. Normal mouse intestinal mucus release requires cystic fibrosis transmembrane regulator-dependent bicarbonate secretion. The Journal of Clinical Investigation. 2009;119(9):2613-2622. DOI: $10.1172 / J C I 38662$

[24] Kristidis P et al. Genetic determination of exocrine pancreatic function in cystic fibrosis. American Journal of Human Genetics. 1992;50(6):1178-1184

[25] Ziegler MM. Meconium ileus. In: Coran AG, Adzick NS, Krummel TM, Laberge J-M, Shamberger RC, Caldamone AA, editors. Pediatric Surgery. 7th ed. Philadelphia, PA: Saunders, an imprint of Elsevier Inc; 2012. pp. 1073-1083

[26] Lee GS, Chandran S, Rajadurai VS. Calcified meconium pseudocyst: $\mathrm{X}$-ray diagnosis of meconium peritonitis at birth. BML Case Reports. July 7, 2015:1

[27] Swischuk LE. Imaging of the Newborn Infant and Young Child. 4th ed. Philadelphia: Lippincott, Williams and Wilkins; 1997

[28] O’Halloran SM, Gilbert J, McKendrick OM, Carty HM, Heaf DP. Gastrografin in acute meconium ileus equivalent. Archives of Disease in Childhood. 1986;61(11):1128-1130

[29] Zangheri G, Andreani M, Ciriello E, Urban G, Incerti M, Vergani P. Fetal intra-abdominal calcifications from meconium peritonitis: Sonographic predictors of postnatal surgery. Prenatal Diagnosis. 2007;27(10):960-963

[30] Noblett HR. Treatment of uncomplicated meconium ileus by Gastrografin enema: A preliminary report. Journal of Pediatric Surgery. 1969;4(2):190-197

[31] Irish M, Bovet PM. Surgical Aspects of Cystic Fibrosis and Meconium Ileus. In: Minkes RK, Chief Editor, 2019. Medscape $($ C, Web MD LLC

[32] Mattei P. Meconium ileus. In: Mattei P, editor. Fundamentals of Pediatric Surgery. New York, NY, USA: Springer Science+Business Media, LLC; 2011. pp. 395-400

[33] Harberg FJ, Senekjian EK, Pokorny WJ. Treatment of uncomplicated meconium ileus via T-tube ileostomy. Journal of Pediatric Surgery. 1981;16(1):61-63

[34] Chappell JS. Management of meconium ileus by resection and end-to-end anastomosis. South African Medical Journal. 1977;52(27):1093-1094

[35] Mabogunje OA, Wang CI, Mahour H. Improved survival of neonates with meconium ileus. Archives of Surgery. 1982;117(1):37-40

[36] Rescorla FJ, Grosfeld JL. Contemporary management of meconium ileus. World Journal of Surgery. 1993;17:381 
Section 3

Hepatobiliary 



\title{
Chapter 5
}

\section{Pediatric Choledochal Cysts: Unknowns are Decreasing}

\author{
Hasan Özkan Gezer
}

\begin{abstract}
Choledochal cysts (CCs) are congenital cystic dilatation of extrahepatic and/ or intrahepatic bile ducts. CCs are more common in Asian population, the cause is still unknown. Although the etiology is controversial, the main elements in the natural historical emergence of the type I and type IV, which make up the majority of all types, have become clearer. The majority of CCs are diagnosed in childhood. Clinical presentation varies from jaundice in young patients to nonspecific abdominal pain in older, but morbidity increases with complications such as cholangitis, pancreatitis, perforation, hepatitis, liver failure, and malignancy in delayed diagnosed patients. MRCP is considered the current gold standard diagnostic modality that is able to accurately assess biliary anatomy. Although the treatment approach has been formed over the years, it still has not reached the last state. Eventually, the removal of the entire cyst and the reconstruction of the remaining biliary tract to drainage is the current treatment approach. But the dilemma is the way of reconstruction procedure (hepaticoduodenostomy or hepaticojejunostomy). All patients should be followed up for a long period of time, regardless of the surgery method.
\end{abstract}

Keywords: choledochal cyst, children, hepaticoduodenostomy, Roux-en-Y hepaticojejunostomy, common bile duct

\section{Introduction}

Choledochal cysts (CCs) are congenital dilatations of extrahepatic and/or intrahepatic bile ducts defined by Vater and Ezler in 1723. It is a rare biliary entity with an estimated incidence of 1:100-150,000 live births in Western countries. In the Asian population, the incidence can be as high as 1:1000 live births. CC is primarily a childhood disease-up to $80 \%$ of patients are diagnosed before 10 years of age. The original classification, first described by Alanso-Lej and colleagues in 1959, was changed in 1977 by Todani and colleagues that classified the CCs as five types. In addition, isolated cystic dilatation of the cystic canal was identified subsequently and proposed as type VI, apart from the revised Todani classification. Although the etiology is controversial, the main elements in the natural historical emergence of the type I and type IV, which make up the majority of all types, have become clearer. A common symptom is nonspecific abdominal pain in older children. When the cyst is complicated, the diagnosis is delayed, the treatment becomes complex, and the results are affected. External drainage (ED), internal drainage (ID), total cyst excision (CE) + hepaticoduodenostomy (HD), and total CE + hepaticojejunostomy (HJ) were defined according to historical development of treatment. Resection is considered that is necessary to prevent further complications and long-term sequel. 


\section{History}

The anatomist Abraham Vater first described the normal anatomy of the bile ducts and the fusiform dilatation of the common bile duct (CBD) in 1723 [1]. Then, Doctor Halliday Douglas first described clinically CBD dilatation in 1852. Douglas had detected a large tenderness cystic mass on the right upper quadrant by physical examination of a 17-year-old girl who had an intermittent right-sided pain, obstructive jaundice, and fever complaints in her history. Despite performing external drainage promptly, she died within 1 month. Subsequently, Douglas detected a CC with her autopsy [2]. In 1894, British surgeon William Swain performed the first successful operation in a 17-year-old girl presented with CC, by anastomosing the jejunum to a giant CC. This patient had been reported 2 months later with no jaundice. In 1922, Golder McWhorter underwent hepaticoduodenostomy after excising the CC in a 49-year-old patient who had complaints since infancy. In 1959, Alanso-Lej and colleagues first published the series of CCs. In this publication, they reviewed 94 cases, published previously, together with their own 2 cases and classified the congenital cystic dilatations of the bile ducts anatomically for the first time [3]. In 1977, Todani and colleagues modified the classification of CCs according to cholangiographic images [4-8].

\section{Incidence}

CCs are more common in Asian populations with an incidence of 1 in 13,000, even 1:1000 in Japan [9], versus 1 in 100,000-150,000 in Western populations [10]. The reason for this Asian preponderance is still unknown [11]. Although predominately diagnosed in children, CCs are found with increasing frequency in adults such that adults comprise the majority of patients in recent series $[12,13]$, which in part may be due to the increased use of diagnostic imaging [14]. In both adult and children [15], females are higher risk for the disease with a nearly $4: 1$ female preponderance $[10,16]$. Nearly, $80 \%$ of CCs are diagnosed in early infancy $[10,17]$.

\section{Pathology}

Grossly, CCs appear as a diffuse dilatation of the bile ducts [18]. In the congenital dilatations of the bile ducts, the cyst wall thickness is between 2 and $7 \mathrm{~mm}$ in diameter and usually involves an inflammatory reaction (80\%) [18] that becomes severe after 10 years of age. Cysts, especially infected, are usually being adherent to the surrounding tissues. Bile ducts and columnar epithelium can be seen in the microscopic examination of the cyst wall. Choledochocele that is covered with duodenal mucosa appears different from other types of CCs according to epithelial histology. Liver biopsy findings usually vary with the age in patients with CC. Although, newborn liver is mostly normal, mild periportal fibrosis may be seen in older children. Varying degrees of histological hepatic changes severity may be seen in most patients with CC when it is diagnosed [19]. Higher degree of liver damage associates with the presence of an anomalous pancreaticobiliary ductal union (APBDU), more severe symptoms, type IVa CC, and younger age [19-21]. It has been observed by investigating liver biopsies that most of these changes resolve after surgical excision; however, preoperative portal fibrosis and central venous distension may remain stable or increase in severity [19]. It is considered that carcinoma of the cyst wall may develop due to recurrent chronic inflammation attacks. 
However, it can also be seen in non-APBDU-associated CC patients. Additionally, the cancer can develop from anywhere such as the cyst wall, the gall bladder, or the common channel junction nearly pancreas, as a consequence of chronic inflammation due to cholangitis $[22,23]$.

\section{Etiology}

Despite the existence of numerous theories and laboratory works to explain the etiology of the disease, the exact etiology remains incompletely understood. Initial theories, put forward in this regard, were congenital weakness of the choledochal wall, distal obstruction, oligoganglionosis, and disturbances in the process of recanalization. However, more accepted theories have been produced parallel to the progress in radiological imaging methods $[24,25]$. Today, there are two main theories that are widely accepted; (1) reflux of trypsin and other pancreatic enzymes to the bile ducts due to an APBDU; (2) obstruction of distal CBD [26-28]. The idea that the choledochal and pancreatic ducts' abnormality about joint and angle was first reviewed by Babbitt in 1969, and subsequently many number of studies, supporting this view, were made [22, 23, 29-32]. A normal pancreaticobiliary junction usually has an acute angle between the CBD and the pancreatic duct [33] and is located within the duodenal wall [34]. The common channel (distance from between the junction of the CBD and the pancreatic duct to ampulla Vater) length is $4 \mathrm{~mm}$ or less, normally. In patients with CCs, this distance (common channel length) increases 5-20 mm [22, 23, 35, 36] that makes the common channel longer [37]. Okada defined it as "common channel syndrome" [36, 38]. It is considered that an abnormal long common channel (especially $>15 \mathrm{~mm}$ proximal to ampullary sphincter) $[39,40]$ causes the pancreatic duct communicating with the choledochal duct without the support of ampulla Vater's circular muscular layer (sphincter of Oddi) [41], which protects the biliary tree from reflux of pancreatic enzymes and bile [34]. Another observation is that the junction angle of the two ducts that should be acute normally, however, is close to $90^{\circ}$ in these patients. It also causes the pancreatic fluids to flow into the CBD due to the higher pressure of pancreatic duct (Figure 1) [41]. Eventually, it is considered that APBDU has a tendency to cause reflux of the pancreatic enzyme into biliary tree with consequent biliary duct inflammation and increased duct pressure, leading to duct wall damage and cystic changes [42, 43]. In animal models of murine APBDU, this mechanism has also been demonstrated $[44,45]$. APBDU is seen in up to $90 \%$ of patients with CC $[25,46]$, compared with $2 \%$ in the general population [10] and this seems to have important clinical implications. In a comparison of APBDU-associated CC versus non-APBDU-associated CC, APBDU-associated CC patients were significantly more likely to have evidence of pathologically confirmed inflammation including hepatitis, cholangitis, and pancreatitis [47, 48]. However, APBDU is not enough to explain the etiology of all CCs, such as diverticular cyst (type II), where the bile ducts were normal except cyst and the cyst is considered to be a sequel of an intrauterine CC rupture, such as meconium pseudocyst [28]. Additionally, type $\mathrm{V}$ CCs are possibly due to dysfunctional remodeling of the ductal plate during embryogenesis [40, 49].

In addition, it is considered that primary strictures of the CBD may also play a role in the development of CCs. The types of the CCs are determined according to the location, severity, and length of the stricture. Detection of these strictures preoperatively is important because treating the CC without addressing the stricture may lead to recurrent episodes of cholangitis. This mechanism has been under estimated and is now believed to be more significant in the pathophysiology of CCs [50]. 


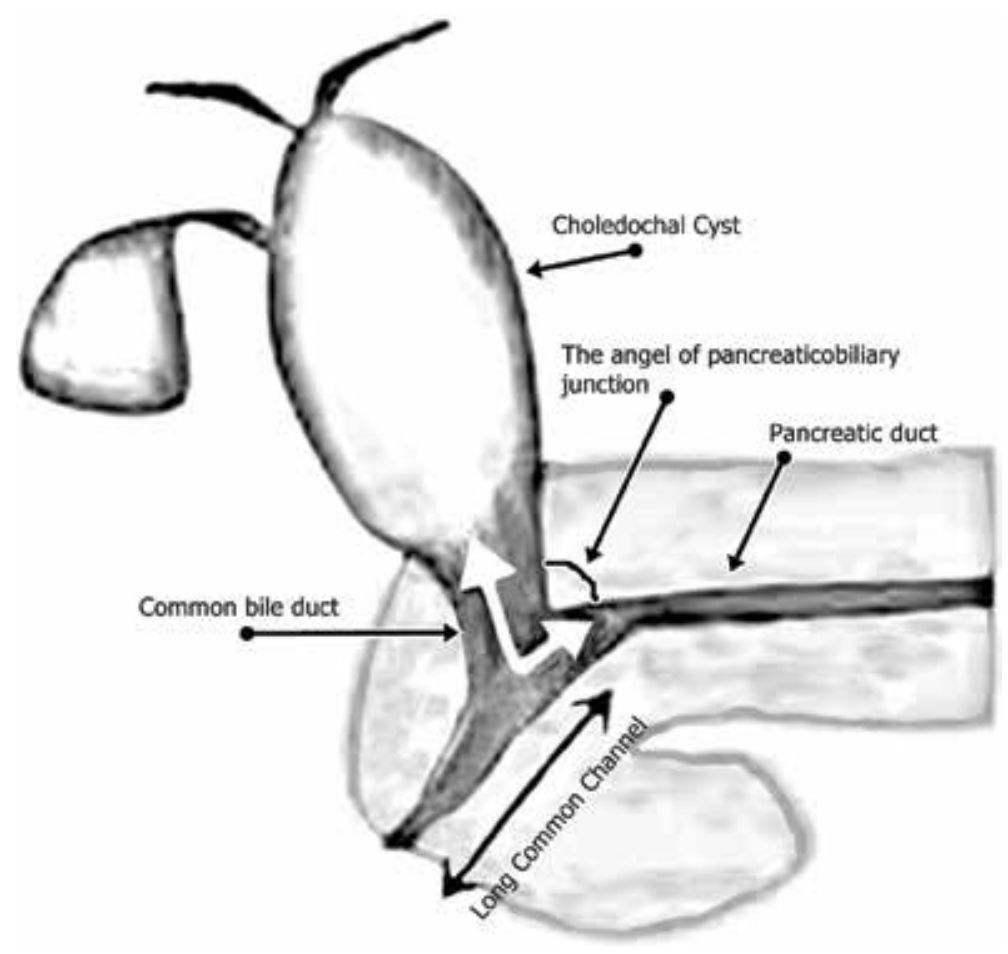

Figure 1.

Anomalous pancreaticobiliary union.

The association of $\mathrm{CC}$ with congenital anomalies remains ambiguous. Previous reports have demonstrated an association of pediatric CC and congenital anomalies. Murphy et al. reported in 2012 that screening for cardiac anomalies may be prudent in CC patients [51]. Other reports have postulated an association of CC with duodenal atresia, colonic atresia, gastroschisis, annular pancreas, and pancreatic cysts $[10,52-57]$.

\section{Classification}

CCs are first classified in 1959 by Alanso-Lej and colleagues [58]. The original classification identified four types of biliary cysts (types I-IV). In 1977, Todani and colleagues [8] modified this classification and added a fifth category of CC, type V biliary cysts or Carol disease (Figure 2) [10]. Apart from types I-V included in the revised Todani classification, isolated cystic dilatation of the cystic duct has been described and suggested as type VI [59].

Type I CC, most commonly seen, $80-90 \%$ of all CC, is a dilatation of the extrahepatic biliary tree. Importantly, the intrahepatic biliary tree is sometimes dilated secondarily due to biliary stasis. Type I cyst can be further subdivided into type Ia, Ib, and Ic cysts $[10,60]$. Type Ia CCs are composed of the gallbladder arising directly from the CC, dilated extrahepatic biliary tree, and a nondilated intrahepatic tree. Type Ib CCs are focal segmental dilatation of the CBD and contain no evidence of APBDU [50, 60]. Finally, type Ic CCs are represented by a fusiform dilatation of the common hepatic duct and CBD in the presence of APBDU $[10,50,60]$, and often also a low-grade stricture at the distal CBD [50]. In type I CC, usually, gallbladder is involved in cyst structure, and cyst extends from hepatic bifurcation to duodenum [61]. Most commonly, the ducts above (right, left, and 

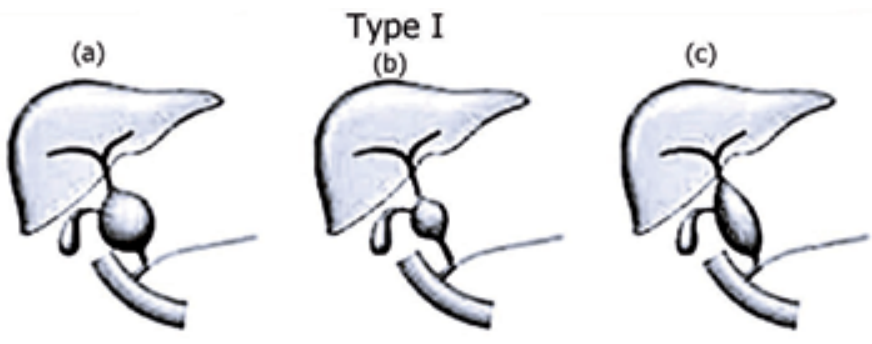

Type II

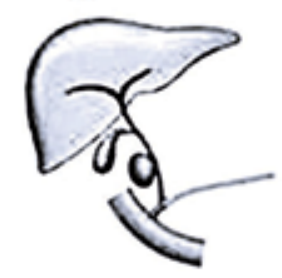

Type IV

(a)
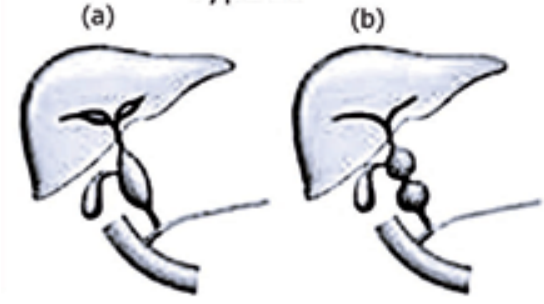

Type III

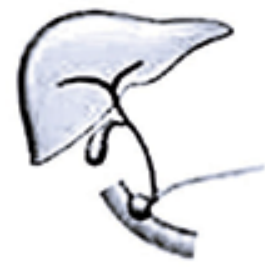

Type V

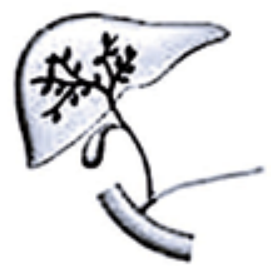

Figure 2.

Types of choledochal cysts, classified by Todani et al.

intrahepatic ducts) and below of the CC are not dilated [14], except type Ic. In type Ic, cyst extends continuously to the common hepatic duct or intrahepatic ducts [62]. Type I CCs, along with type IV cysts, have the highest risk of malignancy [10]. This is not surprising because both of them have extrahepatic involvement associated with APBDU [63].

Type II CCs ( $2 \%$ of all CCs) consist of a diverticular dilatation of the extrahepatic bile duct system and is considered true diverticulum. During the diagnostic cholangiography study, the diverticulum is filled with radiopaque substance and can be confused with the gall bladder duplication seen rarely [10].

Type III CCs (choledochoceles), 4\% of all CCs, are characterized by distal (located at the pancreaticobiliary junction) CBD dilatation, confined to the wall of the duodenum and often bulging into the duodenal lumen [25]. Type III choledochal cysts are dissimilar to other types of CCs, with features such as appearing in both sexes equally and low malignancy incidence $[10,64]$. Cysts are mostly not associated with APBDU. Because of all these characteristics, it has been suggested that type III CCs should not be classified as a type of CCs revised by Todani $[25,64,65]$. In addition, Ziegler et al. reported that choledochoceles occur more frequently in older male patients presenting with acute pancreatitis [64].

Type IV CCs, the second most common (15-20\% of all), are multiple cysts which can involve both the intrahepatic and extrahepatic biliary trees. Type IV CC 
can be further subdivided into type IVa and IVb cysts depending on intrahepatic involvement. Type IVa CC refers to multiple segmental communicating biliary dilatations located in the intra- and extrahepatic biliary tracts, and relative stricture at the junction that is used to distinguish the true type IVa CC from type I [11]. Type IVa CCs are usually associated with APBDU [50]. Type IVb CC refers to multiple extrahepatic biliary cysts without intrahepatic involvement [25]. Some recent studies have shown that intrahepatic ductal dilatations seen on preoperative imaging are thought to have been caused by distal obstruction and not true intrahepatic biliary duct disease [11]. Additionally, the question of "Is the distinguishing type I from type IVa really necessary preoperatively?" has not answered yet. Because distinguishing between types I and IVa CCs is controversial for some authors due to complete excision of the extrahepatic bile ducts, and intensive long-term follow-up still remains standard of care for both types [11].

Type V CC or Carol disease, added by Todani, is characterized by multifocal segmental intrahepatic biliary ductal dilatation [14] without the evidence of extrahepatic dilatation [66]. Caroli disease is uncommon, accounting for less than $10 \%$ of cases. Patients often present in adolescence or early adulthood with recurrent cholangitis, abdominal pain, or jaundice. However, they may present later with the sequel of portal hypertension and cirrhosis [67]. Renal abnormalities, such as medullary sponge kidney, autosomal dominant polycystic kidney disease, and medullary cystic disease can be seen in Caroli disease [49]. Some authors call Caroli disease as Caroli syndrome when congenital hepatic fibrosis is also seen, as in half of the patients $[68,69]$.

Type VI CC, isolated cystic dilatation of the cystic duct, is rare with only several case reports describing it. Although it is not officially part of the revised Todani classification, it has been proposed to be called type VI CC [70]. If the cyst emerges from the cystic duct near a level close to the CBD, it can be confused with type II CCs. In such cases, the relation of the cyst with the cystic duct should be thoroughly evaluated to differentiate them [59].

\section{Clinical presentation}

Type IC cysts are the earliest cysts that can be detected by 15-gestational week fetal ultrasonography [71, 72]. There are two clinical forms of disease: adult and infant. In infant form, symptoms such as obstructive jaundice, clay colored stools, and hepatomegaly make it difficult to distinguish from biliary atresia. Adult form of CCs is also congenital, although they usually remain silent until the age of 2 years. There are three main symptoms in the classical clinical triad: recurrent jaundice $69-75 \%$, right upper quadrant pain $47-60 \%$, and right upper quadrant mass $47-80 \%$. But the classic triad only presents in $10 \%$ of cases $(6-25 \%)[46,73,74]$.

Abdominal pain is the most common symptom (93.8\%) [75], especially in older patients and presents with colic pattern which has a variable interval time (between attacks) up to several years. When investigating the cause of CBD dilatation and differential diagnosis of unclear upper abdominal pain, jaundice, and pancreatitis in children, CCs must be considered [76]. Unfortunately, 29-62\% of pediatric patients with CC have been reported that they have choledocholithiasis $[77,78]$, that is, distinguishing this two situation (CC-associated choledocholithiasis and -nonassociated choledocholithiasis) may be difficult. Choledocholithiasis can also lead to CBD dilatation which can be misdiagnosed as a CC [79].

In $1-2 \%$ of cases, especially in infants, CCs may present with rupture and biliary peritonitis prompting emergency biliary drainage [80, 81]. It is not a surprise in diagnosing pancreatitis in patients with $\mathrm{CC}$, because of association of the presence of APBDU [10, 46, 82]. 
The risk for development of biliary carcinoma in the general population starts after the fourth decade and the incidence increase with age to $0.15 \%$ after the eighth decade. However, the risk for the development of carcinoma in patients with a CC starts in childhood and shows a significant increase with age. Interestingly, the age of biliary carcinoma development in patients who have undergone internal drainage without cyst excision has been reported to be 15 years earlier on average than patients who have never had surgery. This is thought to be associated with intestinal bacterial contamination and pancreatic enzymes added to biliary stasis [83]. The malignancy incidence in resected bile duct material has been reported as $7.5 \%$ for all age groups, and 0.4 and $11.4 \%$ for those under and over the age of 18 , respectively. The incidence has been reported to gradually increase every decade to $38.2 \%$ over the age of 60 , possibly related to chronic inflammation [5]. The incidence of a biliary malignancy development following CC excision is reported as $0.7-5.4 \%$. The malignancy can arise from anywhere such as the porta hepatis, pancreas, or the intrahepatic bile ducts. The time to onset after primary surgical intervention is reported to be 1-34 years. The total excision of the cyst significantly decreases the probability of a malignancy although it does not eliminate it completely $[84,85]$.

\section{Diagnostic evaluation}

Ultimately, multimodality imaging techniques are often utilized including computed tomography (CT), magnetic resonance imaging (MRI), and/or endoscopic retrograde cholangiopancreatography (ERCP) to confirm the extent of ductal involvement or the presence of extrahepatic disease [25]. Frequently, further imaging techniques are used to differentiate type I CC from type IVa, in the presence of intrahepatic biliary dilatation [11]. A cyst, presenting in the porta hepatitis, separated from the gallbladder and continuing with enlarged biliary ducts can be shown by ultrasound (US). Additionally, fusiform dilatation of choledoch, intrahepatic biliary dilatation (60-80\%), biliary stones, and state of liver parenchyma can be shown by US [86].

Other intraabdominal cysts, such as pancreatic pseudocysts, echinococcal cysts, or biliary cystadenomas should also be differentiated from CCs, whether the cyst has continuity with the biliary tree or not [40]. CT is not only useful for demonstrating continuity of the cyst with the biliary tree, but also demonstrates relation of the cyst with the surrounding structures and the presence of associated malignancy [87]. In order to correctly plan surgery, CT cholangiography can be used to identify the full anatomy of the biliary tree but unfortunately it has been reported to be less sensitive for imaging the pancreatic duct which is responsible for the reflux of contrast into the biliary ducts [87]. As it is well known today, the nephrohepatotoxicity of the contrast and the ionized radiation exposed are the restrictions of CT utilization in pediatric population (Figure 3) [62].

MRCP is noninvasive and highly sensitive (70-100\%) and specific (90-100\%) in the diagnosis of CCs $[88,89]$, so, is considered the current gold standard imaging even for initial evaluation [62]. Additionally, there is no irradiation, and modern scanners have alleviated the need for protracted breath-hold making it more amenable to the pediatric population $[10,90]$. Although both ultrasound and CT are highly sensitive and specific in the diagnosis of CCs, MRCP can better identify the CCs subtypes and coexisting abnormalities [89]. For example, MRI can easily identify the pancreaticobiliary ductal anatomy, while ultrasound cannot accurately demonstrate the APBDU $[89,91]$. Additionally, MRCP is preferred modality in the pediatric population due to invasive nature and inherent risks of endoscopic ultrasound and ERCP, despite their ability of detecting the abnormality of the common 

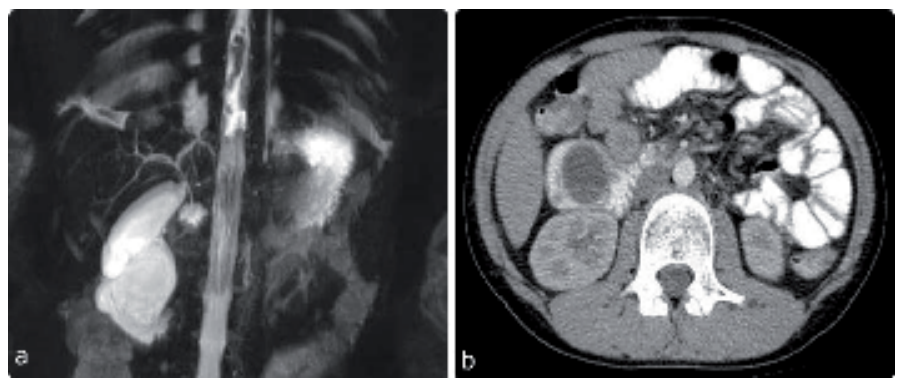

Figure 3.

Type III CC. (a) MRCP; (b) CT image.

channel [89]. MRCP has also been shown to be as effective as intraoperative cholangiography in planning surgery [18]. In addition, lower cost and morbidity compared to other imaging/diagnostic modalities, and reliability for detecting abnormalities associated with CCs such as cholangiocarcinoma and choledocholithiasis, are some of the favorable features of MRCP [89, 92]. Unfortunately, as seen often in patients with CCs, intraductal air, debris, stones, or protein plugs can interfere with the signal and alter visualization (Figure 4) [93].

Although it is highly sensitive, invasiveness and associated risks including cholangitis, bleeding, pancreatitis, and perforation makes percutaneous transhepatic cholangiography (PTC) or ERCP utilization less frequently [94]. Moreover, PTC and ERCP can be technically challenging and require general anesthesia in the pediatric population. A lot of surgeons are finding the use of diagnostic ERCP and PTC in CCs unnecessary due to advantages of both MRCP and perioperative cholangiography (performed in nearly almost patients and give highly detailed information about

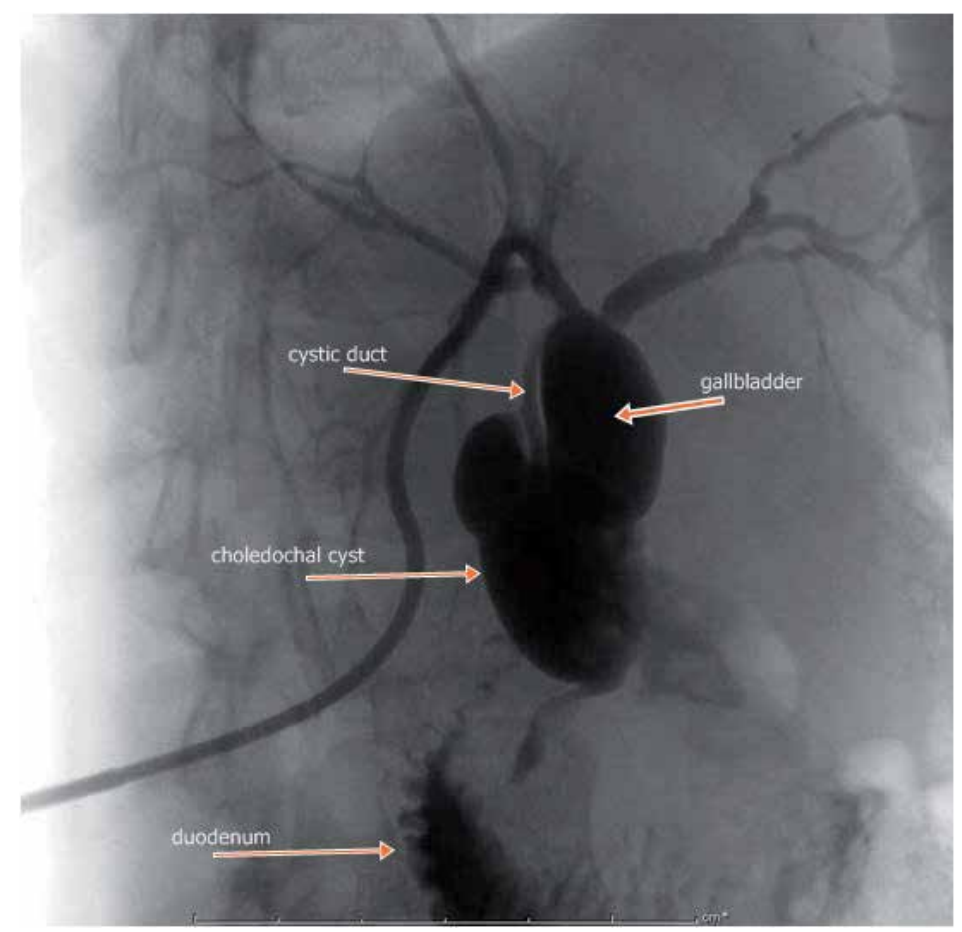

Figure 4.

PTC reveals the detailed anatomy of the biliary tracts and associated CC. 
biliary anatomy) [10, 95, 96]. Contemporary, ERCP should only be performed in cases where the appropriate diagnosis cannot be made by other less-invasive examinations, or when therapeutic performance (complications such as cholangitis or biliary stone obstruction $[97,98]$ and stabilization of the patients with preparing them to the next definitive surgery) is required (Figure 4) $[39,99,100]$.

Another method that is not preferred now for diagnostic purposes is hepatobiliary scintigraphy. Although it is possible to do it with all IDA variants, DISIDA is the best. The radio isotopic substance is collected in the liver and is normally thrown into the biliary tract, but accumulates in the cyst space in patients with CC $[101,102]$.

\subsection{The differential diagnosis}

There are many diseases including biliary atresia, infectious hepatitis, embryonal hepatic rhabdomyosarcoma, biliary lithiasis, pancreatitis, biliary hamartoma in the differential diagnosis of CCs, especially biliary atresia that is one of the two causes of neonatal obstructive jaundice in neonatal period [10]. Differentiating cystic biliary atresia (CBA), a subtype of biliary atresia and has an entirely different treatment approach, from CCs is particularly difficult. Therefore, prompt accurate diagnosis is critical [103]. While earlier presentation (<months of age), smaller cysts with less dilatation of the intrahepatic biliary system, and an atretic gallbladder with irregular and hypoplastic biliary radicles that is seen on ultrasound and cholangiography are characteristics of CBA patients [10, 20, 104, 105]; a dilated gallbladder communicated with the cyst in addition with a dilated intrahepatic biliary tree is mostly a determiner to infantile CC [104].

It is still important to differentiate biliary rhabdomyosarcoma, a rare soft tissue tumor that affects only $1 \%$ of children, from CC $[106,107]$. In the presence of a mass or intraductal growth that causes obstructive jaundice, the possible diagnosis should return in favor of rhabdomyosarcoma in children and prompt evaluation is necessary [107].

"Children with CBD dilatation did not differ significantly in clinical characteristics compared with children who had obstructive CBD dilatation" said Oh and colleagues [78] by evaluating the cholangiographic characteristics of 85 children with CBD dilatation to differentiate obstructive and congenital CBD dilatation. Indeed, it can be difficult to distinguish them. Therefore, in the pediatric population with dilated biliary trees, ruling out a distal biliary obstruction that causes secondary biliary dilatation is prudent and essential [18].

As noted above, type I CC may present with intrahepatic biliary dilatation secondary to biliary stasis, thus resembling a type IVa CC. Some authors consider that this distinction is critical given the therapeutic implications and the need to include hepatic resection (in the case of type IVa CC) in addition to extrahepatic biliary tree excision [18], whereas the others consider that the need preoperatively distinguishing between type I and IVa CCs is controversial because for both, complete excision of the extrahepatic bile duct and intensive long-term follow-up remains standard of care [11].

\section{Management}

The treatment time of antenatal diagnosed of CCs has been still a matter of debate. Some reports say that they can be operated within 2-6 weeks, even if they are asymptomatic, due to a potential complication risk of cysts, whereas, the others suggest that they can be followed-up for a time of period with US and regular monitoring of liver functions $[108,109]$. 
Surgical treatment of CCs should be performed electively except complicated ones such as cyst perforation. Children, who have acute problems related to liver and pancreas, should be initially received appropriate medical treatment to remove inflammation and associated obstruction at the pancreaticobiliary system before surgery [110].

In the first half of this century, treatment methods such as cyst aspiration, marsupialization, and external drainage (cholecystostomy, tube drainage) had been used more extensively in the surgical treatment of CCs. And as expected, high mortality and morbidity rates had been detected in patient's follow-up period. Surgical methods such as partial cyst excision and cystoduodenostomy were defined between 1920 and 1930 [111, 112]. Especially, cystoduodenostomy has been the preferred method by many surgeons until the early 1970s [113,114] when the long-term morbidity was detected as higher (30-50\%) [7]. Therefore, in those years, Roux-en-Y cystojejunostomy had identified with the idea of preventing the reflux of the duodenal contents into the bile ducts $[7,30,115]$. Indeed, the cholangitis had been significantly reduced with this method, but not completely eliminated [28]. After soon, it had been demonstrated that anastomosis with a large stoma, as possible as (at least $4 \mathrm{~cm}$ ) is more important for protecting reflux-related cholangitis attacks than which intestinal segment it is performed (duodenum or jejunum) [116]. The recognition of the cancer development from the left cystic wall and Babbitt's APBDU theory made the idea of cyst excision popular soon after. It had been reported that carcinoma develops after the internal drainage procedures at a frequency of 2.5-17.5\% several years later as a consequence of chronic inflammation of the cyst wall. Therefore, cyst excision had gain popularity in a very short time [116].

Contemporarily, the definitive treatment for CCs are total excision that has become preferred management strategy over the internal drainage procedures (choledochocystoduodenostomy or choledochocystojejunostomy), which have an only historical value today despite they had been used as a treatment method in the past although caused high morbidity (probably because of not relieving biliary stasis sufficiently) [117, 118]. Furthermore, only complete resection can fully decrease the risk of malignant degeneration: a critical point in the pediatric population with a large number of expectant life years. The general aim is to remove the cyst completely and restore biliary enteric drainage either into the duodenum by hepaticoduodenostomy (HD) or jejunum by Roux-en-Y hepaticojejunostomy (RYHJ), although specific approaches for types vary minimally. Surgical intervention should be elective and patients should be medically optimized prior to operative intervention. If patient has a cholangitis or pancreatitis attack preoperatively, the infection should be adequately treated with broad-spectrum intravenous antibiotics or biliary decompression if needed [118].

Surgery for CC disease can be performed open or laparoscopically based on patient characteristics and surgeon preference.

$\mathrm{HD}$ and RYHJ are the two most commonly utilized techniques of reconstruction [119], although other replacement conduits such as jejunal interposition HD, valved jejunal interposition HD, nonrefluxing biliary appendicoduodenostomy, hepaticoenterostomy, and wide hilar hepaticojejunostomy have been reported [120-125]. HD has been favored by some groups [126, 127] but most series suggests significantly more bile reflux compared with RYHJ [121], which is currently the most commonly utilized reconstruction.

\subsection{Open surgery}

In all cases, cholangiography should be performed initially to obtain detailed anatomical information about the intra- and extrahepatic bile ducts, irrespective of 
preoperative examinations. Dissection of extrahepatic bile ducts starts from the gallbladder. The terminal end of the cyst opening to the duodenum should be isolated, clamped, cut, and transfixed, firstly. Some surgeons suggest that dissection should be continued until the appearance of pancreatic ducts, while the others not suggest. Additionally, some surgeons taking into account that dissection toward the lower end of the cyst may cause inevitable unplanned pancreatic duct injury that pancreaticoduodenectomy requirement should be in your mind, although very rarely [75]. After the distal portion of the cyst is ligated and cut, the posterior wall is dissected from the surface of the portal vein. In cases of marked inflammation, the cyst may be excised by leaving the posterior wall on the portal vein. The dissection should go on till the hepatic hilus. The best strategy to obtain a wide anastomosis stoma is to make a hepatic dissection more proximally until the left hepatic duct is seen. Although all parts of CCs need to be removed, sometimes residual proximal cyst walls can be left to facilitate biliary anastomosis [75]. Dilated bile ducts should be irrigated with heparinized saline to clear the gallstones before anastomosis. After the cyst is excised, one of the hepaticoenterostomy methods, such as hepaticojejunostomy, HD, jejunal interposition HD, valved jejunal interposition HD, nonrefluxing biliary appendicoduodenostomy, hepaticoenterostomy, and wide hilar hepaticojejunostomy [120-125] is performed for biliary reconstruction. In $\mathrm{RYHJ}, 40-\mathrm{cm}$ jejunal loop replaces to the hepatic hilus. In RYHJ surgery, to avoid the elongation of a blind pouch as the child grows, an end-to-end anastomosis of the jejunum to the CBD is recommended if technically possible [128]. If an end-to-side anastomosis is required (in some cases, the bile duct is too small), it should be as close as possible to the closed end of the jejunal limb. Additionally, although it is not possible to predetermine the length of the Roux limb, it should be appropriate to the child's overall bowel length considering future growth. In HD, anastomosis is performed between the duodenum second part and the bile duct. The duodenum was mobilized to a limit. The duodenum is anchored to the liver at porta to avoid tension on the anastomosis (Figure 5).

The intraabdominal drain, kept in Morison's pouch, may be removed on the seventh postoperative day [124].
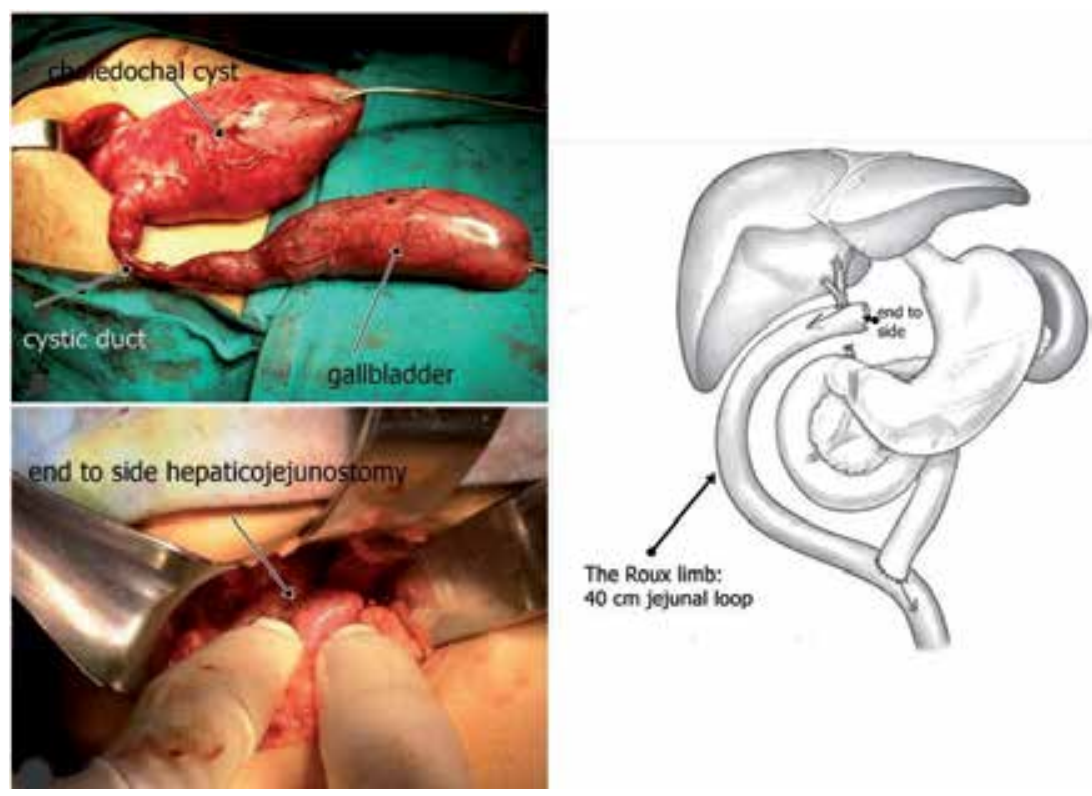

Figure 5.

Intraoperative pictures and drawn cartoon showing of the procedure. 


\subsection{Laparoscopic surgery}

Laparoscopic treatment of choledochal cysts was first described in 1995 [129] and demonstrated that it could be performed in children as young as 3 months [130] and as small as $6 \mathrm{~kg}$ [131]. As with most surgical diseases, longer operating time and shorter hospital stay [132] were comparable with open surgical approaches, and in the absence of cholangitis or pancreatitis, it becomes more suitable treatment [132]. While four or five ports are typically used in the traditional laparoscopic approach $[130,133]$, the use of single-port laparoscopy [134] and robotic surgical system [135] has also been reported. In a prospective randomized study of 121 children undergoing laparoscopic cyst excision with RYHJ, routine postoperative drainage has been shown to be unnecessary [136].

\subsection{The optimal technique for biliary reconstruction}

The most commonly performed operations for biliary reconstruction after complete surgical resection of CCs are RYHJ or HD [124]. There is a debate regarding the optimal technique for biliary reconstruction [124]. RYHJ is considered as an ideal technique for the repair of CC, but HD has gained wide acceptance and favored by many surgeons open as well as laparoscopically because of its advantages over hepaticojejunostomy. HD is more physiologic, but theoretically, the closeness of hepaticoenterostomy to stomach makes HD to have greater chance of cholangitis and bile gastritis, but in a meta-analyzed study [137], it has been shown that while the incidence of bile gastritis after HD is even higher when examined endoscopically, interestingly, there is no difference of cholangitis between HD and RYHJ. Additionally, HD is simpler to perform and associated with fewer complications such as adhesive bowel obstruction, anastomotic leakage, and peptic ulcer as compared to RYHJ [138]. HD requires less operative time, allows faster recovery of bowel function, and produces fewer complications requiring reoperation [139]. If there is an anastomotic stricture following HD, it can be easily managed by endoscopy as against hepaticojejunostomy [124]. But, when the diameter of the common hepatic duct more than $10 \mathrm{~mm}$ that lets duodenal contents more likely to reflux easily into the intrahepatic bile ducts through the HD anastomosis or when the intrahepatic biliary dilatation is present that lets refluxed duodenal contents remain longer in the intrahepatic bile ducts, HD is not recommended because of higher risk of cholangitis or anastomotic stricture formation [124]. Some studies have demonstrated high incidence of secondary bile reflux proven by endoscopy after HD [121]. Recently, a patient with hilary bile duct carcinoma, who was performed HD for the biliary reconstruction at the age of 13 months, has been reported in the 19 years follow-up after the primary cyst excision. Reflux of duodenal contents (including activated pancreatic enzymes) into the intrahepatic bile ducts through the HD anastomosis is thought to be hazardous to the bile duct mucosa in this patient [124]. Adhesive bowel obstruction is seen with a higher incidence in RYHJ that comprises a Roux-en-Y jejuna limb and two anastomoses, compared with HD. Cholangitis, peptic ulcer, fat malabsorption, diarrhea, and malnutrition are the other complications [126]. A significant incidence of long-term complications requiring reoperation such as anastomosis stenosis has been observed with the follow-up studies of patients who underwent hepaticojejunostomy after cyst excision $[138,140]$, and a wide hilar hepaticojejunostomy extending into the left hepatic duct is advocated for the way to prevent it [125].

\subsection{Treatment specifically for types}

Treatment of type I CC includes excision of the extrahepatic biliary tract, cholecystectomy, and reconstruction of the biliary system. If the duct is dilated at the 
distal margin, the mucosa may be left behind to prevent damage to the pancreaticobiliary system and can be striped. Infrequently, because of recurrent episodes of the cholangitis, the cyst wall may densely adherent to the portal vein, precluding safe resection [141]. In such cases, resection of the anterior wall with careful fulguration of the mucosa of the posterior wall can be performed [141]. Hepatic bifurcation is carefully evaluated for stricture and inflammation before performing anastomosis during proximal transection. If one of them is seen, more proximal transection should be considered [18].

For type II CCs, mostly, diverticulectomy or simple cyst excision is enough for the treatment. Primary or over a T-tube closure can be performed, and reconstruction is occasionally required if there is significant luminal narrowing [18].

One of the methods such as endoscopic sphincterotomy, sphincteroplasty, sphincteroplasty with cyst excision, or pancreaticoduodenectomy may be used to manage pediatric patients with type III CCs (choledochoceles) [10, 25]. Various reports denote adequate symptom control with this approach $[142,143]$; however, long-term follow-up is lacking. Cysts not amenable to endoscopic intervention may benefit from lateral duodenotomy with sphincteroplasty and unroofing or marsupialization of the cavity [18].

Type IV CC is approached differently based on the presence or absence and location of intrahepatic disease [15]. Type IVb cysts are treated in the same fashion as type I. Management for IVa disease differs due to the presence of intra- as well as extrahepatic involvement, as well as the presence of functional liver disease. Of foremost importance is the characterization of actual type IVa as opposed to type I with upstream ductal dilatation due to stasis and functional obstruction [50]. If the dilatation is anatomic and isolated (limited; i.e., left hemiliver), partial hepatectomy with reconstruction to the remaining hepatic ducts may be warranted due to the ongoing risk of malignant transformation in the intrahepatic biliary system [85]. However, not all patients are appropriate candidates for partial hepatectomy [144]. Those patients with obvious dilatations and stenosis of intrahepatic ducts, intrahepatic duct stones, or parenchymal atrophy may benefit from hepatectomy [144]. If hepatectomy is planned concomitantly with extrahepatic duct excision, the distribution should allow removal of all disease (of the vast majority of the severe disease) with adequate future liver remnant [15]. If the pattern is more diffuse or imaging is inconclusive, treatment in a type I paradigm with close postoperative surveillance to follow intrahepatic ducts has been utilized [145]. This approach is justified by studies demonstrating that patients who progress to malignancy most commonly develop extrahepatic cholangiocarcinoma or gallbladder cancer (approximately $85 \%$ of malignancy), whereas intrahepatic cholangiocarcinoma rarely occurs [144]. It is reported that the intrahepatic component has actually resolved in 3-6 months with adequate drainage [146]. To differentiate type IVa from type I, while in adults, preoperative percutaneous biliary drainage to decompress the intrahepatic biliary ductal system has been advocated [147], in children, this practice has not been reported probability due to the difficulty in maintaining the external tube. Although long-term results are not known, intrahepatic cystojejunostomy, in addition to hepaticojejunostomy, has been described as a way of preventing liver resection in type IVa cysts [148]. Complete extrahepatic excision with hepaticoenterostomy and drainage of the remaining cyst externally or internally should adequately ameliorate biliary stasis in the presence of bilobar unresectable intrahepatic cyst [18].

Management of patients with Caroli's disease can be particularly difficult given the location of the cysts and frequent necessity for surgery (considerable potential for cholangitis, liver complications, and biliary cirrhosis; moderate potential for neoplasia (7\%)) [15]. In Caroli disease, intrahepatic cysts can be seen as limited 
disease restricted to a single segment/lobe or diffuse disease involving the entire intrahepatic biliary tree. If the patient has not developed cirrhosis and portal hypertension, the unilobar cystic disease should be treated with anatomic hepatectomy and biliary enteric bypass. However, bilobar disease should be treated with symptom-directed nonoperative treatment methods as including litholytic agents such as ursodiol, antibiotics, and percutaneous drainage if possible. Close follow-up is required for malignant transformation. Although there is no identification for prophylactically orthotopic liver transplantation in the treatment of the disease, it should be kept in mind for the choice of the treatment in patients who have diffuse symptomatic disease with cirrhosis or portal hypertension [149].

\subsection{Follow-up}

Patients should be monitored every 6 months during the postoperative 3 years and then annually. On initial follow-up, while all patients should be evaluated with complete blood count, liver function tests and abdominal US, on subsequent followup, investigations are done only in symptomatic ones. Long-term follow-up can be made by visits, telephonic conversations, and postal inquiry [124].

\section{Outcomes and results}

Resection of pediatric CC is generally well tolerated [18]. Despite recent advances in surgical techniques and perioperative management, short- and long-term complications are not rare in children, while they are more common in adults [6]. Complications such as recurrent cholangitis attacks, malignant transformation, intracystic or intrahepatic gallstone formation, cirrhosis development, and pancreatitis are common in patients who are not operated on. Complications such as anastomotic leakage, gastrointestinal or intraabdominal bleeding, acute pancreatitis, pancreatic leakage, wound infection, wound dehiscence, intraabdominal infection/abscess, intussusception can be seen in early postoperative period defined as short-term complications [150]. Most early complications can be treated conservatively [151].

However, most series are without early mortality and report rates of acute complications including wound infections from 0 to $17 \%$, without significant difference between infants and children $[46,132]$.

Surgical inexperience and severe inflammation are often implicated in the development of anastomotic bile leakage [151]. The diagnosis of bile leakage is difficult and delayed in some cases due to nonspecific symptoms [151]. It may not always be possible to differentiate with the imaging findings of US and CT because not all of the intraabdominal fluid collection after surgery is associated with bile leakage, and so, this late diagnosis may result in mortality due to septicemia and septic shock [151]. However, it is reported that MRCP can be used to diagnose and accurately localize the site of bile leakage noninvasively [7, 151]. Bile leaks in the hepaticooenterostomy line can self-limited within a few weeks if they can be drained externally. If the bowel movement is sufficient during this period, the child can be fed by enterally. If the extracted bile is given back to the stomach with NG catheter, electrolyte losses can also be prevented. The bilirubin level of the child may remain high due to edema in the anastomosis line within the first 2 weeks, even if the operation has been successfully performed. If this takes longer, biliary tree, even anastomosis, can be evaluated with PTC [110]. Reoperation is considered only after the failure of conservative treatment [151]. The leakage can be repaired by a circumferential buried suture around the anastomotic site, peritoneal lavage, and effective drainage [151]. 
Gastrointestinal bleeding may be due to hepaticojejunostomy or stress ulcer.

Acute pancreatitis occurs in patients with CC, both preoperatively and due to injury of the pancreatic tissue during distal dissection of the cyst or to edema in the distal part of the pancreatic duct related postoperatively (4.2\%) [151]. Therefore, it has been recovered with a conservative treatment for a short time period. Some reports say that $\mathrm{CC}$ excision without ligation of the distal stenotic stump decreases the incidence of pancreatic duct injury [75]. A probe inserted into the pancreatic duct through a duodenotomy may help to prevent pancreatic duct injury in difficult cases [75]. Additionally, Urushihara et al. [152] consider that using bipolar electro cautery to scrape pancreatic tissue away from the bile duct wall during the dissection of the intrapancreatic part of the bile duct causes minimal bleeding and enables clear identification of the narrow part of the CBD [151]. Eventually, complete resection of the distal portion of the cyst, removal of debris and protein plugs in the long common channel and pancreatic duct, and correction of anomalous arrangement of the pancreaticobiliary duct junction are essential to minimize pancreatic complications after the operations [151]. The pancreatic fistula occurs because of not closing the distal choledoch well after the cyst excision or injured pancreatic duct during the dissection. An external drainage of 3-4 weeks allows the fistulas closing.

Late/long-term complications (5-15\%) [151] include anastomotic stricture, cholangitis, hepatolithiasis, ileus, cirrhosis, and malignancy. Benign anastomotic stricture with recurrent cholangitis is less common than in adults but is still seen in many as $10-25 \%$ of patients and can be associated with both intrahepatic and bile duct stone formation [117].

After intraabdominal surgery, small bowel obstructions, mostly due to adhesions, are common [154]. Patients should be closely monitored for any possible clinical deterioration [154]. If there is no improvement after $48 \mathrm{~h}$ of follow-up, there is a high risk of bowel resection due to bowel necrosis despite it has not been clearly defined [156]. Furthermore, it has been defined that during biliary reconstruction, the length and placement of the Roux loop is very important in adhesive bowel obstruction developing postoperatively [156].

In terms of anastomotic stricture, improvement of surgical skills, preservation of blood supply, no or mild inflammation cyst wall, and construction of wide (larger than $1 \mathrm{~cm}$ ) and tension-free stoma are key factors to reduce anastomosisrelated complication [138]. There should be no delay in surgical or endoscopic intervention once biliary obstruction develops postoperatively, but a great deal of planning and a thoughtful workup are required [151]. Kim et al. [155] reported that PTC with stone removal and balloon dilatation was useful in patients with anastomotic stricture. However, some investigators consider that recurrent anastomotic strictures may occur due to fibrosis even after balloon dilatation with PTCS, and repeated cholangitis may cause multiple intrahepatic biliary strictures, recurrent hepatic stones, and development of biliary carcinoma $[152,153]$. Hence, especially in young patients, it is recommended that revision of the hepaticojejunostomy followed by ductoplasty, to create a wide stoma for sufficient bile drainage [152, 153].

Rigorous long-term follow-up after pediatric CC resection is limited, but the risk of biliary carcinoma (cholangiocarcinoma, squamous cell carcinoma, sarcoma, gallbladder cancer $[12,157]$, most often cholangiocarcinoma) clearly remains elevated even after CC excision compared to the general population [18]. The malignancy risk is considered to increase with age at surgery, and the cumulative biliary malignancy risk 25 years after primary surgery has been reported to be as high as $11 \%$ [75]. Malignant disease has been noted in up to $14 \%$ of patients after CC resection as a child [46]. In fact, cancer is the most frequent cause of late mortality in pediatric CC series [18]. Even after complete excision, patients are at higher risk for malignancy than general population [158]. Continued surveillance is, therefore, 
strongly recommended, though it is not known whether there are risk factors such as retained portion of cyst or not [18]. In those with known malignancy, oncologic principles should apply; patients who can undergo safe resection with negative margins are appropriate for operation [15]. Resection may include hepatectomy with regional lymphadenectomy, extirpation of extrahepatic bile ducts with regional lymphadenectomy (and cholecystectomy), or pancreaticoduodenectomy [15]. However, the 5-year survival rate for patients with CCs complicated by malignancy is high, up to $55 \%$ in patients with cholangiocarcinoma [159].

\section{Author details}

Hasan Özkan Gezer

Department of Pediatric Surgery, Başkent University Faculty of Medicine, Ankara, Turkey

*Address all correspondence to: hozkangezer@yahoo.com.tr

\section{IntechOpen}

(C) 2019 The Author(s). Licensee IntechOpen. This chapter is distributed under the terms of the Creative Commons Attribution License (http://creativecommons.org/licenses/ by/3.0), which permits unrestricted use, distribution, and reproduction in any medium, provided the original work is properly cited. (cc) BY 


\section{References}

[1] Ando H, Ito T, Watanabe Y, Seo T, Kaneko K, Nagaya M. Spontaneous perforation of choledochal cyst. Journal of the American College of Surgeons. 1995;181(2):125-128

[2] Douglas AH. Case of dilatation of common bile duct. Monthly Journal of Medical Science (London). 1852;14:97

[3] Altın MA, Büyükpamukçu N, Hiçsönmez A. Çocukluk çağı koledok kistlerinin cerrahi tedavisi. Hacettepe Tip Dergisi. 1985;18:186

[4] Caroli J. Diseases of intrahepatic bile ducts. Israel Journal of Medical Sciences. 1968;4(1):21-35

[5] Flanigan DP. Biliary cysts. Annals of Surgery. 1975;182:635-643

[6] Klotz D, Cohn BD, Kottmeier PK. Choledochal cysts: Diagnostic and therapeutic problems. Journal of Pediatric Surgery. 1973;8(2):271-283

[7] O’Neill JA Jr, Clatworthy HW Jr. Management of choledochal cysts: A fourteen-year follow-up. American Journal of Surgery. Apr 1971;37(4):230-237

[8] Todani T, Watanabe Y, Narusue M, Tabuchi K, Okajima K. Congenital bile duct cysts: Classification, operative procedures, and review of thirty-seven cases including cancer arising from choledochal cyst. American Journal of Surgery. 1977;134(2):263-269

[9] O’Neill JA Jr. Choledochalcyst. Current Problems in Surgery. 1992;29(6):361-410

[10] Soares KC, Arnaoutakis DJ, Kamel I, Rastegar N, Anders R, Maithel S, et al. Choledochal cysts: Presentation, clinical differentiation, and management. Journal of the American College of
Surgeons. 2014;219(6):1167-1180. DOI: 10.1016/j.jamcollsurg.2014.04.023 [Epub Jun 27, 2014]

[11] Gezer HÖ, Oğuzkurt P, Temiz A, İnce E, Ezer SS, Hiçsönmez A. Choledochal cysts in children: Intrahepatic ductal dilatation does not indicate true intrahepatic biliary duct disease. Turkish Journal of Gastroenterology. Jan 2016;27(1):23-29. DOI: 10.5152/ tjg.2015.150211 [Epub Nov 27, 2015]

[12] Edil BH, Cameron JL, Reddy S, Lum Y, Lipsett PA, Nathan H, et al. Choledochal cyst disease in children and adults: A 30-year singleinstitution experience. Journal of the American College of Surgeons. 2008;206(5):1000-1005; discussion 10058. DOI: 10.1016/j.jamcollsurg.2007.12.045 [Epub Mar 17, 2008]

[13] Xia HT, Yang T, Liang B, Zeng JP, Dong JH. Treatment and outcomes of adults with remnant intrapancreatic choledochal cysts. Surgery.

2016;159(2):418-425. DOI: 10.1016/j. surg.2015.04.042 [Epub Jun 27, 2015]

[14] Lewis VA, Adam SZ, Nikolaidis P, Wood C, Wu JG, Yaghmai V, et al. Imaging of choledochal cysts. Abdominal Imaging. 2015;40(6):1567-1580.

DOI: $10.1007 / \mathrm{s} 00261-015-0381-4$

[15] Ronnekleiv-Kelly SM, Soares KC, Ejaz A, Pawlik TM. Management of choledochalcysts. Current Opinion in Gastroenterology. 2016;32(3):225-231. DOI: 10.1097/ MOG.0000000000000256

[16] Lipsett PA, Pitt HA. Surgical treatment of choledochal cysts. Journal of Hepato-Biliary-Pancreatic Sciences. 2003;10(5):352-359

[17] Wiseman K, Buczkowski AK, Chung SW, Francoeur J, Schaeffer D, Scudamore CH. Epidemiology, 
presentation, diagnosis, andoutcomes of choledochalcysts in adults in an urban environment. American Journal of Surgery. 2005;189(5):527-531; discussion 531

[18] Soares KC, Goldstein SD, Ghaseb MA, Kamel I, Hackam DJ, Pawlik TM. Pediatric choledochal cysts: Diagnosis and current management. Pediatric Surgery International. 2017;33(6):637-650. DOI: 10.1007/ s00383-017-4083-6 [Epub Mar 31, 2017]

[19] Sugandhi N, Agarwala S, Bhatnagar V, Singh MK, Sharma R. Liverhistology in choledochal cyst-pathological changes and response to surgery: The over looked aspect? Pediatric Surgery International. 2014;30(2):205-211. DOI: $10.1007 / \mathrm{s} 00383-013-3453-\mathrm{y}$

[20] Zhou LY, Guan BY, Li L, Xu ZF, Dai $\mathrm{CP}$, Wang W, et al. Objective differential characteristics of cysticbiliary atresia and choledochal cysts in neonates and young infants: Sonographic findings. Journal of Ultrasound in Medicine. 2012;31(6):833-841

[21] Fumino S, Higuchi K, Aoi S, Furukawa T, Kimura O, Tajiri T.Clinic alanalysis of liver fibrosis in choledochal cyst.Pediatric Surgery International 2013;29(11):1097-1102. DOI: $10.1007 /$ s00383-013-3368-7

[22] Guelrud M, Morera C, Rodriguez M, Prados JG, Jaén D. Normal and anomalous pancreaticobiliary union in children and adolescents. Gastrointestinal Endoscopy. 1999;50(2):189-193

[23] Iwai N, Yanagihara J, Tokiwa K, Shimotake T, Nakamura K. Congenital choledochal dilatation with emphasis on pathophysiology of the biliary tract. Annals of Surgery. 1992;215(1):27-30

[24] Kusunoki M, Saitoh N, Yamamura T, Fujita S, Takahashi T, Utsunomiya
J. Choledochal cysts. Oligoganglionosis in the narrow portion of the choledochus. Archives of Surgery. 1988;123(8):984-986

[25] Martin RF. Biliary cysts: A review and simplified classification scheme. Surgical Clinics of North America. 2014;94(2):219-232. DOI: 10.1016/j. suc.2014.01.011

[26] Miyano T, Suruga K, Suda K. Abnormal choledocho-pancreatico ductal junction related to the etiology of infantile obstructive jaundice diseases. Journal of Pediatric Surgery. 1979;14(1):16-26

[27] Miyano T, Tsunoda S, suruga $\mathrm{K}$, et al. A proposal of choledochopancreatic long common channnel disease. Japanese Journal of Pediatric Surgery. 1979;11:1111

[28] O’Neill JA Jr. Choledochal cyst. In: O’Neill JA Jr, Rowe MI, Grosfeld JL, Fonkalsrud EW, Coran AG, editors. Pediatric Surgery. 5th ed. Vol. 2. St Louis, Missouri; Mosby-Year Book, Inc; 1998; pp. 1483-1494

[29] Arima E, Akita H. Congenital biliary tract dilatation and anomalous junction of the pancreatico-biliary ductal system. Journal of Pediatric Surgery. 1979;14(1):9-15

[30] Babbitt DP. Congenital choledochal cysts: New etiological concept based on anomalous relationships of the common bile duct and pancreatic bulb. Annales de Radiologie (Paris) 1969;12(3):231-240

[31] Ikeda N. Experimental and clinical studies on anomalous relationship in the pancreaticobiliary duct. The Tokushima Journal of Experimental Medicine. 1978;25(1-2):47-57

[32] Ito T, Ando H, Nagaya M, Sugito $\mathrm{T}$. Congenital dilatation of the common bile duct in children. The etiologic significance of the narrow segment 
distal to the dilated common bile duct. Zeitschrift für Kinderchirurgie. 1984;39(1):40-45

[33] Lee HK, Park SJ, Yi BH, Lee AL, Moon JH, Chang YW. Imaging features of adult choledochal cysts: A pictorial review. Korean Journal of Radiology. 2009;10(1):71-80. DOI: 10.3348/ kjr.2009.10.1.71

[34] Beltrán MA. Current knowledge on pancreaticobiliary reflux in normal pancreaticobiliary junction. International Journal of Surgery. 2012;10(4):190-193. DOI: 10.1016/j. ijsu.2012.02.009 [Epub Feb 20, 2012]

[35] Jona JZ, Babbitt DP, Starshak RJ, LaPorta AJ, Glicklich M, Cohen RD. Anatomic observations and etiologic and surgical considerations in choledochal cyst. Journal of Pediatric Surgery. 1979;14(3):315-320

[36] Okada A, Oguchi Y, Kamata S, Ikeda Y, Kawashima Y, Saito R. Common channel syndromeDiagnosis with endoscopic retrograde cholangiopancreatography and surgical management. Surgery. 1983;93(5):634-642

[37] Li L, Yamataka A, Yian-Xia W, Da-Yong W, Segawa O, Lane GJ, et al. Ectopic distal location of the papilla of Vater in congenital biliary dilatation: Implications for pathogenesis. Journal of Pediatric Surgery. 2001;36(11):1617-1622

[38] Okada A, Nakamura T, Higaki J, Okumura K, Kamata S, Oguchi Y. Congenital dilatation of the bile duct in 100 instances and its relationship with anomalous junction. Surgery, Gynecology \& Obstetrics. 1990;171(4):291-298

[39] Liu QY, Nguyen V. Endoscopic approach to the patient with congenital anomalies of the biliary tract. Gastrointestinal Endoscopy Clinics of
North America. 2013;23(2):505-518. DOI: 10.1016/j.giec.2012.12.004 [Epub Jan 17, 2013]

[40] Levy AD, Rohrmann CA Jr. Biliary cystic disease. Current Problems in Diagnostic Radiology. 2003;32(6):233-263

[41] Iwai N, Tokiwa K, Tsuto T, et al. sphincter function of the bile duct in patients with congenital choledochal dilatation. Journal of the Japanese Society of Pediatric Surgeons. 1986;22:688

[42] Søreide K, Søreide JA. Bile duct cyst as precursor to biliary tract cancer. Annals of Surgical Oncology. 2007;14(3):1200-1211 [Epub Dec 23, 2006]

[43] Chang J, Jang JY, Kang MJ, Jung W, Shin YC, Kim SW. Clinicopathologic differences in patients with gallbladder cancer according to the presence of anomalous biliopancreatic junction. World Journal of Surgery. 2016;40(5):1211-1217. DOI: $10.1007 /$ s00268-015-3359-z

[44] Yamashiro Y, Miyano T, Suruga K, Shimomura H, Suda K, Matsumoto $M$, et al. Experimental study of the pathogenesis of choledochal cyst and pancreatitis, with special reference to the role of bile acids and pancreatic enzymes in the anomalous choledochopancreatico ductal junction. Journal of Pediatric Gastroenterology and Nutrition. 1984;3(5):721-727

[45] Miyano T, Suruga K, Suda K. “The choledocho-pancreatic long common channel disorders" in relation to the etiology of congenital biliary dilatation and other biliary tract disease. Annals of the Academy of Medicine, Singapore. 1981;10(4):419-426

[46] de Vries JS, de Vries S, Aronson DC, Bosman DK, Rauws EA, Bosma A, et al. Choledochal cysts: Age of 
presentation, symptoms, and late complications related to Todani's classification. Journal of Pediatric Surgery. 2002;37(11):1568-1573

[47] Park SW, Koh H, Oh JT, Han SJ, Kim S. Relationship between anomalous pancreaticobiliary ductal union and pathologic inflammation of bile duct in choledochal cyst. Pediatric Gastroenterology, Hepatology \& Nutrition. 2014;17(3):170-177. DOI: 10.5223/pghn.2014.17.3.170 [Epub Sep 30, 2014]

[48] Song HK, Kim MH, Myung SJ, Lee SK, Kim HJ, Yoo KS, et al. Choledochal cyst associated the with anomalous union of pancreaticobiliary duct (AUPBD) has a more grave clinical course than choledochal cyst alone. The Korean Journal of Internal Medicine. 1999;14(2):1-8

[49] Fulcher AS, Turner MA, Sanyal AJ. Case 38: Caroli disease and renal tubular ectasia. Radiology. 2001;220(3):720-723

[50] Todani T, Watanabe Y, Toki A, Morotomi Y. Classification of congenital biliary cystic disease: Special reference to type Ic and IVA cysts with primary ductal stricture. Journal of Hepato-Biliary-Pancreatic Surgery. 2003;10(5):340-344

[51] Murphy AJ, Axt JR, Lovvorn HN 3rd. Associations between pediatric choledochal cysts, biliary atresia, and congenital cardiac anomalies. Journal of Surgical Research. 2012;177(2):e59-e63. DOI: 10.1016/j.jss.2012.04.018 [Epub May 1, 2012]

[52] Iwai A, Hamada Y, Takada K, Inagaki $\mathrm{N}$, Nakatake R, Yanai $\mathrm{H}$, et al. Choledochal cyst associated with duodenal atresia: Case report and review of the literature. Pediatric Surgery International. 2009;25(11):995-998. DOI: $10.1007 /$ s00383-009-2462-3
[53] Shih HS, Ko SF, Chaung JH. Is there an association between duodenal atresia and choledochal cyst? Journal of Pediatric Gastroenterology and Nutrition. 2005;40(3):378-381

[54] Nijagal A, Ozgediz D, Feldstein VA, Lee H, Harrison MR. Colonic atresia and choledochal cyst: A rare combination. Pediatric Surgery International. 2009;25(1):113-115. DOI: $10.1007 /$ s00383-008-2280-z [Epub Nov 5, 2008]

[55] Komuro H, Takahashi MI, Matoba K, Hori T, Hirai M, Gotoh C, et al. Rare association of severe hypoplasia of the abdominal aorta with imperforate anus, colonic atresia, and choledochal cyst. Pediatric Surgery International. 2006;22(3):289-292 [Epub Nov 26, 2005]

[56] Serber J, Stranzinger E, Geiger JD, Teitelbaum DH. Association of gastroschisis and choledochal cyst. Journal of Pediatric Surgery. 2009;44(3):e23-e26. DOI: 10.1016/j. jpedsurg.2008.12.005

[57] Komuro H, Makino S, Tahara K. Choledochal cyst associated with duodenal obstruction. Journal of Pediatric Surgery. 2000;35(8):1259-1262

[58] Alonso-lej F, Rever WB Jr, Pessagno DJ. Congenital choledochal cyst, with a report of 2, and an analysis of 94, cases. International Abstract of Surgery. 1959;108(1):1-30

[59] Serena Serradel AF, Santamaría Linares E, Herrera Goepfert R. Cystic dilatation of the cystic duct: A new type of biliary cyst. Surgery. 1991;109

(3 Pt 1):320-322

[60] Rozel C, Garel L, Rypens F, Viremouneix L, Lapierre C, Décarie JC, et al. Imaging of biliary disorders in children. Pediatric Radiology. 2011;41(2):208-220. DOI: 10.1007/ s00247-010-1829-x [Epub Sep 24, 2010] 
[61] Spier LN, Crystal K, Kase DJ, Fagelman D, Spier N. Choledochocele: Newer concepts of origin and diagnosis. Surgery. 1995;117(4):476-478

[62] Drabek J, Keil R, Stovicek J, Lochmannova J, Hlava S, Snajdauf J, et al. The role of endoscopic retrograde cholangiopancreatography in choledochal cysts and/or abnormal pancreatobiliary junction in children. Przegląd Gastroenterologiczny. 2017;12(4):303-309. DOI: 10.5114/ pg.2017.72107 [Epub Dec 14, 2017]

[63] Li L, Yamataka A, Wang YX, Wang DY, Wang K, Li ZX, et al. Anomalous pancreatic duct anatomy, ectopic distal location of the papilla of Vater and congenital biliary dilatation: A new developmental triad? Pediatric Surgery International. 2003;19(3):180-185 [Epub Apr 15, 2003]

[64] Ziegler KM, Pitt HA, Zyromski NJ, Chauhan A, Sherman S, Moffatt D, et al. Choledochoceles: Are they choledochal cysts? Annals of Surgery. 2010;252(4):683-690. DOI: 10.1097/ SLA.0b013e3181f6931f

[65] Ziegler KM, Zyromski NJ. Choledochoceles: Are they choledochal cysts? Advances in Surgery. 2011;45:211-224

[66] Moslim MA, Gunasekaran G, Vogt D, Cruise M, Morris-Stiff G. Surgical management of Caroli's disease: Single center experience and review of the literature. Journal of Gastrointestinal Surgery. 2015;19(11):2019-2027. DOI: 10.1007/s11605-015-2918-9 [Epub Aug $25,2015]$

[67] Brancatelli G, Federle MP, Vilgrain V, Vullierme MP, Marin D, Lagalla R. Fibropolycystic liver disease: CT and MR imaging findings. Radiographics. 2005;25(3):659-670

[68] Mabrut JY, Bozio G, Hubert C, Gigot JF. Management of congenital bile duct cysts. Digestive Surgery. 2010;27(1):
12-18. DOI: 10.1159/000268109 [Epub Apr 1, 2010]

[69] Santiago I, Loureiro R, CurvoSemedo L, Marques C, Tardáguila F, Matos C, et al. Congenital cystic lesions of the biliary tree. American Journal of Roentgenology. 2012;198(4):825-835. DOI: 10.2214/AJR.11.7294

[70] Bode WE, Aust JB. Isolated cystic dilatation of the cystic duct. American Journal of Surgery. 1983;145(6):828-829

[71] Dewbury KC, Aluwihare AP, Birch SJ, Freeman NV. Prenatal ultrasound demonstration of a choledochal cyst. The British Journal of Radiology. 1980;53(633):906-907. DOI: 10.1259/0007-1285-53-633-906

[72] Howell CG, Templeton JM, Weiner S, Glassman M, Betts JM, Witzleben CL. Antenatal diagnosis and early surgery for choledochal cyst. Journal of Pediatric Surgery. 1983;18(4):387-393

[73] Goon HK, Tai A, Samad SA. congenital bile duct dilatation: Review of 35 cases. Pediatric Surgery International. 1992;7:332

[74] Miyano T, Yamataka A, Kato Y, Segawa O, Lane G, Takamizawa S, et al. Hepaticoenterostomy after excision of choledochal cyst in children: A 30-year experience with 180 cases. Journal of Pediatric Surgery. 1996;31(10):1417-1421

[75] Gadelhak N, Shehta A, Hamed H. Diagnosis and management of choledochal cyst: 20 years of single center experience. World Journal of Gastroenterology. 2014;20(22): 7061-7066. DOI: 10.3748/wjg.v20. i22.7061

[76] Kim YA, Kim GM, Chun P, Hwang EH, Mun SW, Lee YJ, et al. Management of pediatric patients presenting with acute abdomen accompanying dilatation of the common bile duct. Pediatric Gastroenterology, Hepatology \& 
Nutrition. 2018;21(3):203-208. DOI: 10.5223/pghn.2018.21.3.203 [Epub Jun $28,2018]$

[77] Huang CS, Huang CC, Chen DF. Choledochal cysts: Differences between pediatric and adult patients. Journal of Gastrointestinal Surgery. 2010;14(7):1105-1110. DOI: $10.1007 /$ s11605-010-1209-8 [Epub Apr 27, 2010]

[78] Oh SH, Chang SH, Kim HJ, Cho JM, Hwang JH, Namgoong JM, et al. Cholangiographic characteristics of common bile duct dilatation in children. World Journal of Gastroenterology. 2015;21(20):6229-6235. DOI: $10.3748 /$ wjg.v21.i20.6229

[79] Youn HS, Seo JH, Park CH, Cho JM, Park JJ. An infantile case of cholelithiasis initially misdiagnosed as choledochal cyst. Pediatrics International. 2012;54(1):168-169. DOI: 10.1111/j.1442-200X.2011.03546.x

[80] Yamaguchi M. Congenital choledochal cyst. Analysis of 1,433 patients in the Japanese literature. American Journal of Surgery. 1980;140(5):653-657

[81] Yamoto M, Urushihara N, Fukumoto K, Miyano G, Nouso $\mathrm{H}$, Morita K, et al. Usefulness of laparoscopic cholecystostomy in children with complicated choledochal cyst. Asian Journal of Endoscopic Surgery. 2015;8(2):153-157. DOI: 10.1111/ases.12170 [Epub Feb 11, 2015]

[82] Diao M, Li L, Cheng

W. Coagulopathy in a subtype of choledochal cyst and management strategy. World Journal of Gastroenterology. 2014;20(30): 10606-10612. DOI: 10.3748/wjg.v20. i30.10606

[83] Makin E, Davenport M. Understanding choledochal malformation. Archives of Disease in Childhood. 2012;97(1):69-72. DOI: 10.1136/adc.2010.195974 [Epub Mar 27, 2011]

[84] Ohashi T, Wakai T, Kubota M, Matsuda Y, Arai Y, Ohyama T, et al. Risk of subsequent biliary malignancy in patients undergoing cyst excision for congenital choledochal cysts. Journal of Gastroenterology and Hepatology. 2013;28:243-247

[85] He XD, Wang L, Liu W, Liu $\mathrm{Q}, \mathrm{Qu} \mathrm{Q}, \mathrm{Li} \mathrm{BL}$, et al. The risk of carcinogenesis in congenital choledochal cyst patients: An analysis of 214 cases. Annals of Hepatology. 2014;13:819-826

[86] Hernanz-Schulman M, Ambrosino MM, Freeman PC, Quinn CB. Common bile duct in children: Sonographicdimensions. Radiology. 1995;195(1):193-195

[87] Lam WW, Lam TP, Saing H, Chan FL, Chan KL. MR cholangiography and CT cholangiography of pediatric patients with choledochal cysts. American Journal of Roentgenology. 1999;173(2):401-405

[88] Huang CT, Lee HC, Chen WT, Jiang CB, Shih SL, Yeung $\mathrm{CY}$. Usefulness of magnetic resonance cholangiopancreatography in pancreatobiliary abnormalities in pediatric patients. Pediatrics $\&$ Neonatology. 2011;52(6):332-336. DOI: 10.1016/j.pedneo.2011.08.006 [Epub Nov 6, 2011]

[89] Sacher VY, Davis JS, Sleeman D, Casillas J. Role of magnetic resonance cholangiopancreatography in diagnosing choledochal cysts: Case series and review. World Journal of Radiology. 2013;5(8):304-312. DOI: 10.4329/wjr.v5.i8.304

[90] Suzuki M, Shimizu T, Kudo T, Suzuki R, Ohtsuka Y, Yamashiro $\mathrm{Y}$, et al. Usefulness of nonbreathhold 1-shot magnetic resonance 
cholangiopancreatography for the evaluation of choledochal cyst in children. Journal of Pediatric Gastroenterology and Nutrition. 2006;42(5):539-544

[91] Guo WL, Huang SG, Wang J, Sheng M, Fang L. Imaging findings in 75 pediatric patients with pancreaticobiliary maljunction: A retrospective case study. Pediatric Surgery International. 2012;28(10): 983-938 [Epub Aug 15, 2012]

[92] Murphy AJ, Axt JR, Crapp SJ, Martin CA, Crane GL, Lovvorn HN 3rd. Concordance of imaging modalities and cost minimization in the diagnosis of pediatric choledochal cysts. Pediatric Surgery International. 2012;28(6): 615-621. DOI: 10.1007/s00383-0123089-3 [Epub Apr 21, 2012 ]

[93] Arshanskiy Y, Vyas PK. Type IV choledochal cyst presenting with obstructive jaundice: Role of MR cholangiopancreatography in preoperative evaluation. American Journal of Roentgenology. 1998;171(2):457-459

[94] Saito T, Terui K, Mitsunaga T, Nakata M, Kuriyama Y, Higashimoto $\mathrm{Y}$, et al. Role of pediatric endoscopic retrograde cholangiopancreatography in an era stressing less-invasive imaging modalities. Journal of Pediatric Gastroenterology and Nutrition. 2014;59(2):204-209. DOI: 10.1097/ MPG.0000000000000399

[95] Park DH, Kim MH, Lee SK, Lee SS, Choi JS, Lee YS, et al. Can MRCP replace the diagnostic role of ERCP for patients with choledochal cysts? Gastrointestinal Endoscopy. 2005;62(3):360-366

[96] Sharma AK, Wakhlu A, Sharma SS. The role of endoscopic retrograde cholangiopancreatography in the management of choledochal cysts in children. Journal of Pediatric Surgery. 1995;30(1):65-67
[97] Schaefer JF, Kirschner HJ, Lichy M, Schlemmer HP, Schick F, Claussen CD, et al. Highly resolved free-breathing magnetic resonance cholangiopancreatography in the diagnostic workup of pancreaticobiliary diseases in infants and young childrenInitial experiences. Journal of Pediatric Surgery. 2006;41(10):1645-1651

[98] Moyer V, Freese DK, Whitington

PF, Olson AD, Brewer F, Colletti

$\mathrm{RB}$, et al. North American Society for Pediatric Gastroenterology, Hepatology and Nutrition. Guideline for the evaluation of cholestatic jaundice in infants: Recommendations of the North American Society for Pediatric Gastroenterology, Hepatology and Nutrition. Journal of Pediatric Gastroenterology and Nutrition. Aug 2004;39(2):115-128

[99] De Angelis P, Foschia F, Romeo E, Caldaro T, Rea F, di Abriola GF, et al. Role of endoscopic retrograde cholangiopancreatography in diagnosis and management of congenital choledochal cysts: 28 pediatric cases. Journal of Pediatric Surgery. 2012;47(5):885-888. DOI: 10.1016/j. jpedsurg.2012.01.040

[100] Otto AK, Neal MD, Slivka AN, Kane TD. An appraisal of endoscopic retrograde cholangiopancreatography (ERCP) for pancreaticobiliary disease in children: Our institutional experience in 231 cases. Surgical Endoscopy. 2011;25(8):2536-2540. DOI: 10.1007/s00464-011-1582-8 [Epub Feb 27, 2011]

[101] Dooms GC, Fisher MR, Higgins $\mathrm{CB}$, Hricak H, Goldberg HI, Margulis AR. MR imaging of the dilated biliary tract. Radiology. 1986;158(2):337-341

[102] Riddlesberger MM Jr. Evaluation of the gastrointestinal tract in the child: CT, MRI, and isotopic studies. Pediatric Clinics of North America. 1988;35(2):281-310 
[103] Hill SJ, Clifton MS, Derderian SC, Wulkan ML, Ricketts RR. Cystic biliary atresia: A wolf in sheep's clothing. The American Surgeon. 2013;79(9): 870-872

[104] Vijayaraghavan P, Lal R, Sikora SS, Poddar U, Yachha SK. Experience with choledochal cysts in infants. Pediatric Surgery International. 2006;22(10): 803-807 [Epub Sep 1, 2006]

[105] Kim WS, Kim IO, Yeon KM, Park KW, Seo JK, Kim CJ. Choledochal cyst with or without biliary atresia in neonates and young infants: US differentiation. Radiology. 1998;209(2):465-469

[106] Nakib G, Calcaterra V, Goruppi I, Romano P, Raffaele A, Schleef J, et al. Robotic-assisted surgery approach in a biliary rhabdomyosarcoma misdiagnosed as choledochal cyst. Rare Tumors. 2014;6(1):5173. DOI: 10.4081/rt.2014.5173 [eCollection Jan 23, 2014]

[107] Elwahab MA, Hamed H, Shehta A, Ali M, Zalata K. Hepatobiliaryrhabdomyosarcoma mimicking choledochal cyst: Lessons learned. International Journal of Surgery Case Reports. 2014;5(4): 196-199. DOI: 10.1016/j.ijscr.2014.01.020 [Epub Feb 7, 2014]

[108] Büyükyavuz I, Ekinci S, Ciftçi AO, Karnak I, Senocak ME, Tanyel FC, et al. A retrospective study of choledochal cyst: Clinical presentation, diagnosis and treatment. The Turkish Journal of Pediatrics. 2003;45(4):321-325

[109] Stringer MD, Dhawan A, Davenport M, Mieli-Vergani G, Mowat AP, Howard ER. Choledochal cysts: Lessons from a 20 year experience. Archives of Disease in Childhood. 1995;73(6):528-531

[110] Todani T, Watanabe Y, Toki A, Urushihara N. Carcinoma related to choledochal cysts with internal drainage operations. Surgery, Gynecology \& Obstetrics. 1987;164(1):61-64

[111] Gross RE. Idiopathic dilatation of the common bile duct in children. The Journal of Pediatrics. 1933;3:730

[112] Mcworther GL. Congenital cystic dilatation of the common bile duct. Archives of Surgery. 1924;8:604

[113] Fonkalsrud EW, Boles

ET. Choledochal cyst in infancy and childhood. Surgery, Gynecology \& Obstetrics. 1965;121:733

[114] Madden JD, Gruwez JA, Tan PY. Obstructive (surgical) jaundice: an analysis of 140 consequtive cases and a consideration of choledocoduodenostomy in its treatment. American Journal of Surgery. 1965;174:711

[115] Spitz L. Choledochal cyst. Surgery, Gynecology \& Obstetrics. 1978;147(3):444-452

[116] Başaklar AC. Bebek ve Çocukların Cerrahi ve Ürolojik Hastalıkları. Turkey: Palme yayıncılık; 2006. ISBN: 975-8982-83-4

[117] Schier F, Clausen M, Kouki M, Gdanietz K, Waldschmidt J. Late results in the management of choledochal cysts. European Journal of Pediatric Surgery. 1994;4(3):141-144

[118] Todani T, Watanabe Y, Toki A, Urushihara N, Sato Y. Reoperation for congenital choledochal cyst. Annals of Surgery. 1988;207(2):142-147

[119] Wei MF, Qi BQ, Xia GL, Yuan JY, Wang G, Weng YZ, et al. Use of the appendix to replace the choledochus. Pediatric Surgery International. 1998;13(7):494-496

[120] Todani T, Watanabe Y, Mizuguchi T, Fujii T, Toki 
A. Hepaticoduodenostomy at the hepatic hilum after excision of choledochal cyst. American Journal of Surgery. 1981;142(5):584-587

[121] Shimotakahara A, Yamataka A, Yanai T, Kobayashi $H$, Okazaki T, Lane GJ, et al. Rouxen-Y hepaticojejunostomy or hepaticoduodenostomy for biliary reconstruction during the surgical treatment of choledochal cyst: Which is better? Pediatric Surgery International. 2005;21(1):5-7

[122] Narasimha Rao KL, Mitra SK, Kochher R, Thapa BR, Nagi B, Katariya $S$, et al. Jejunal interposition hepaticoduodenostomy for choledochal cyst. The American Journal of Gastroenterology. 1987;82(10):1042-1045

[123] Shamberger RC, Lund DP, Lillehei CW, Hendren WH 3rd. Interposed jejunal segment with nipple valve to prevent reflux in biliary reconstruction. Journal of the American College of Surgeons. 1995;180(1):10-15

[124] Patil V, Kanetkar V, Talpallikar MC. Hepaticoduodenostomy for biliary reconstruction after surgical resection of choledochal cyst: A 25-year experience. Indian Journal of Surgery. 2015;77(Suppl 2):240-244. DOI: 10.1007/s12262-012-0783-2 [Epub Dec 18, 2012]

[125] Stringer MD. Wide hilar hepaticojejunostomy: The optimum method of reconstruction after choledochal cyst excision. Pediatric Surgery International. 2007;23(6): 529-532 [Epub Apr 17, 2007]

[126] Schimpl G, Aigner R, Sorantin E, Mayr J, Sauer H. Comparison of hepaticoantrostomy and hepaticojejunostomy for biliary reconstruction after resection of a choledochal cyst. Pediatric Surgery International. 1997;12(4):271-275
[127] Yeung F, Chung PH, Wong KK, Tam PK. Biliary-enteric reconstruction with hepaticoduodenostomy following laparoscopic excision of choledochal cyst is associated with better postoperative outcomes: A singlecentre experience. Pediatric Surgery International. 2015;31(2):149-153. DOI: 10.1007/s00383-014-3648-x [Epub Nov 30, 2014]

[128] Miyano T. Choledochal cysts. In: Stringer MD, Oldham KT, Mouriquand PDE, editors. Pediatric Surgery and Urology: Long-term Outcomes. Cambridge: Cambridge University Press. pp. 465-479

[129] Farello GA, Cerofolini A, Rebonato M, Bergamaschi G, Ferrari C, Chiappetta A. Congenital choledochal cyst: Video-guided laparoscopic treatment. Surgical Laparoscopy Endoscopy. 1995;5(5):354-358

[130] Le DM, Woo RK, Sylvester K, Krummel TM, Albanese CT. Laparoscopic resection of type 1 choledochal cysts in pediatric patients. Surgical Endoscopy. 2006;20(2):249-251 [Epub Dec 28, 2005]

[131] Lee JH, Kim SH, Kim HY, Choi YH, Jung SE, Park KW. Early experience of laparoscopic choledochal cyst excision in children. Journal of Korean Surgical Society. 2013;85(5):225-229. DOI: 10.4174/jkss.2013.85.5.225 [Epub Oct 25, 2013]

[132] Liuming H, Hongwu Z, Gang L, Jun J, Wenying H, KKY W, et al. The effect of laparoscopic excision vs open excision in children with choledochal cyst: A midterm follow-up study. Journal of Pediatric Surgery. 2011;46(4):662-665. DOI: 10.1016/j. jpedsurg.2010.10.012

[133] Margonis GA, Spolverato G, Kim Y, Marques H, Poultsides G, Maithel S, et al. Minimally invasive resection of choledochal cyst: A 
feasible and safe surgical option. Journal of Gastrointestinal Surgery. 2015;19(5):858-865. DOI: 10.1007/ s11605-014-2722-y [Epub Dec 18, 2014]

[134] Diao M, Li L, Cheng W. To drain or not to drain in Roux-en-Y hepatojejunostomy for children with choledochal cysts in the laparoscopic era: A prospective randomized study. Journal of Pediatric Surgery. 2012;47(8):1485-1489. DOI: 10.1016/j. jpedsurg.2011.10.066

[135] Chang EY, Hong YJ, Chang HK, Oh JT, Han SJ. Lessons and tips from the experience of pediatric robotic choledochal cyst resection. Journal of Laparoendoscopic \& Advanced Surgical Techniques: Part A. 2012;22(6):609-614. DOI: 10.1089/lap.2011.0503 [Epub Jun 13, 2012]

[136] Diao M, Li L, Li Q, Ye M, Cheng $W$. Single-incision versus conventional laparoscopic cyst excision and Roux-Y hepaticojejunostomy for children with choledochal cysts: A case-control study. World Journal of Surgery. 2013;37(7):1707-1713. DOI: $10.1007 /$ s00268-013-2012-y

[137] Narayanan SK, Chen

Y, Narasimhan KL, Cohen

RC. Hepaticoduodenostomy versus hepaticojejunostomy after resection of choledochal cyst: A systematic review and meta-analysis. Journal of Pediatric Surgery. 2013;48(11):2336-2342. DOI: 10.1016/j.jpedsurg.2013.07.020

[138] Todani T, Watanabe Y, Urushihara $\mathrm{N}$, Noda T, Morotomi Y. Biliary complications after excisional procedure for choledochal cyst. Journal of Pediatric Surgery. 1995;30(3):478-481

[139] Santore MT, Behar BJ, Blinman TA, Doolin EJ, Hedrick HL, Mattei $\mathrm{P}$, et al. Hepaticoduodenostomy vs hepaticojejunostomy for reconstruction after resection of choledochal cyst. Journal of Pediatric Surgery.
2011;46(1):209-213. DOI: 10.1016/j.

jpedsurg.2010.09.092

[140] Yamataka A, Ohshiro K, Okada Y, Hosoda Y, Fujiwara T, Kohno S, et al. Complications after cyst excision with hepaticoenterostomy for choledochal cysts and their surgical management in children versus adults. Journal of Pediatric Surgery. 1997;32(7):1097-1102

[141] Senthilnathan P, Patel ND, Nair AS, Nalankilli VP, Vijay

A, Palanivelu C. Laparoscopic management of choledochal cysttechnical modifications and outcome analysis. World Journal of Surgery. 2015;39(10):2550-2556. DOI: 10.1007/ s00268-015-3111-8

[142] Saeki I, Takahashi Y, Matsuura T, Takahata S, Tanaka M, Taguchi T. Successful endoscopic unroofing for a pediatric choledochocele. Journal of Pediatric Surgery. 2009;44(8): 1643-1645. DOI: $10.1016 /$ j.jpedsurg. 2009.03.042

[143] Dohmoto M, Kamiya T, Hünerbein M, Valdez H, Ibanegaray J, Prado J. Endoscopic treatment of a choledochocele in a 2-yearold child. Surgical Endoscopy. 1996;10(10):1016-1018

[144] Lee SE, Jang JY, Lee YJ, Choi DW, Lee WJ, Cho BH, Korean Pancreas Surgery Club et al. Choledochal cyst and associated malignant tumors in adults: A multicenter survey in South Korea. Archives of Surgery. 2011;146(10):1178-1184. DOI: 10.1001/ archsurg.2011.243

[145] Acker SN, Bruny JL, Narkewicz MR, Roach JP, Rogers A, Karrer

FM. Preoperative imaging does not predict intrahepatic involvement in choledochal cysts. Journal of Pediatric Surgery. 2013;48(12):2378-2382. DOI: 10.1016/j. jpedsurg.2013.08.008 
[146] Joseph VT. Surgical techniques and long-term results in the treatment of choledochal cyst. Journal of Pediatric Surgery. 1990;25(7):782-787

[147] Savader SJ, Venbrux AC, Benenati JF, Mitchell SE, Widlus DM, Cameron JL, Osterman FA Jr. Choledochal cysts: Role of noninvasive imaging, percutaneous transhepatic cholangiography, and percutaneous biliary drainage in diagnosis and treatment. Journal of Vascular and Interventional Radiology 1991;2(3):379-385

[148] Urushihara N, Fukumoto K, Fukuzawa H, Tani M, Matsuoka T, Suzuki K, et al. Hepaticojejunostomy and intrahepatic cystojejunostomy for type IV-A choledochal cyst. Journal of Pediatric Surgery. 2007;42(10):1753-1756

[149] Shi LB, Peng SY, Meng XK, Peng $\mathrm{CH}$, Liu YB, Chen XP, et al. Diagnosis and treatment of congenital choledochal cyst: 20 years' experience in China. World Journal of Gastroenterology. 2001;7(5):732-734

[150] Fujishiro J, Urita Y, Shinkai T, Gotoh C, Hoshino N, Ono K, et al. Clinical characteristics of liver fibrosis in patients with choledochal cysts. Journal of Pediatric Surgery. 2011;46 (12):2296-2300. DOI: 10.1016/j. jpedsurg.2011.09.017

[151] Sheng Q, Lv Z, Xu W, Xiao X, Liu J, Wu Y. Reoperation after cyst excision with hepaticojejunostomy for choledochal cysts: Our experience in 18 cases. Medical Science Monitor. 2017;23:1371-1377

[152] Urushihara N, Fukumoto K, Fukuzawa H, Mitsunaga M, Watanabe $\mathrm{K}$, Aoba T, et al. Long-term outcomes after excision of choledochal cysts in a single institution: Operative procedures and late complications. Journal of Pediatric Surgery. Dec
2012;47(12):2169-2174. DOI: 10.1016/j. jpedsurg.2012.09.001

[153] Edil BH, Olino K, Cameron $\mathrm{JL}$. The current management of choledochal cysts. Advances in Surgery. 2009; 43:221-232

[154] Lautz TB, Barsness KA. Adhesive small bowel obstruction-Acute management and treatment in children. Seminars in Pediatric Surgery. 2014;23(6):349-352. DOI: 10.1053/j. sempedsurg.2014.06.006 [Epub Jun 4, 2014]

[155] Kim JH, Choi TY, Han JH, Yoo BM, Kim JH, Hong J, et al. Risk factors of postoperative anastomotic stricture after excision of choledochal cysts with hepaticojejunostomy. Journal of Gastrointestinal Surgery. May 2008;12(5):822-828. [Epub Dec 6 2007]

[156] Yamataka A, Kobayashi H, Shimotakahara A, Okada Y, Yanai $\mathrm{T}$, Lane GJ, et al. Recommendations for preventing complications related to Roux-en-Y hepaticojejunostomy performed during excision of choledochal cyst in children. Journal of Pediatric Surgery. 2003;38(12):1830-1832

[157] Ono S, Fumino S, Shimadera S, Iwai N. Long-term outcomes after hepaticojejunostomy for choledochal cyst: A 10- to 27-year follow-up. Journal of Pediatric Surgery. 2010;45(2):376-378. DOI: 10.1016/j. jpedsurg.2009.10.078

[158] Cho MJ, Hwang S, Lee YJ, Kim KH, Ahn CS, Moon DB, et al. Surgical experience of 204 cases of adult choledochal cyst disease over 14 years. World Journal of Surgery. 2011;35(5):1094-1102. DOI: $10.1007 /$ s00268-011-1009-7

[159] Lee KF, Lai EC, Lai PB. Adult choledochal cyst. Asian Journal of Surgery. 2005;28(1):29-33 

Section 4

Abdominal Wall Defects 



\title{
Management of Gastroschisis
}

\author{
Alaa Obeida and Aly Shalaby
}

\begin{abstract}
Gastroschisis (GS) is one of the congenital abdominal wall defects, in which the bowel has prolapsed without a covering through a defect adjacent to (and nearly always to the right of) an otherwise normal umbilicus. Proper management of such cases gives them the opportunity to survive and thrive. In this chapter, simplified flowcharts for the initial management of GS, surgical intra-operative decisions and post-operative active follow-up of such cases will be presented and discussed. The first flowchart will discuss how to deal with a GS case from birth till the operative theatre, while the second flowchart will take the lead to guide the surgeon with the available surgical options and how to choose the suitable one for the case. Finally, the post-operative active follow-up fluid management and possible complications are discussed.
\end{abstract}

Keywords: gastroschisis, AWDs, fluid management, LMIC, complications

\section{Introduction}

Gastroschisis (GS) or more aptly "laparoschisis" is a congenital abdominal wall defect (AWD) leading to herniation of the gut more commonly to the right of the umbilical cord (Figure 1). It differs from other AWDs in causality, risk factors, and associated anomalies [1-3].

GS incidence is increasing worldwide [4, 5] and is estimated around 1 in 2200 live births [6,7]. Antenatal scans detect most cases [8], survival in developed countries is excellent [7] and apart from some gastrointestinal dysfunction, longterm problems are rare [9].

This chapter is dedicated to discuss in simplified flowchart-form the initial, operative and post-operative management of GS with emphasis on low-resource settings. In addition it aims to outline salient topics such as fluid management and complications.

\section{Etiology and embryology}

Though unexplained, a young maternal age and low socioeconomic status are the commonest risk factors for GS [10,11]. Smoking, drugs, environmental toxins and poor nutrition have also been implicated [12]. A genetic link in the form of homozygous gene polymorphisms has been reported [13] and is substantiated by an increased prevalence among familial cases of birth defects and twins [14].

The embryological origin of GS is still a matter of conjecture. Several theories have been put forward attempting to expound the abdominal wall defect: failed body-wall folding [15]; a vascular insult to the omphalo-mesenteric artery [16] or to the right umbilical vein [17]; a localized disruption of the amniotic membrane [18] 


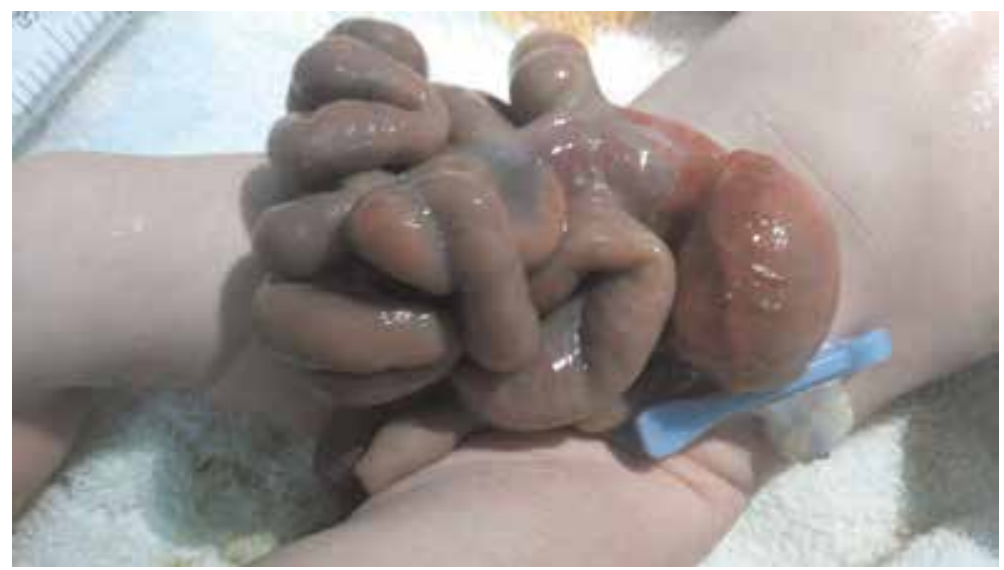

Figure 1.

Gastroschisis with prolapsed bowel to the right of the umbilicus.

or teratogen-induced mesenchymal failure [19]. None of the theories are fully satisfactory [20]. The right-sided occurrence of the defect has been linked to the position of the yolk sac $[15,21]$ without clear reasoning as to why. Left-sided defects have also been described [22].

\section{Antenatal diagnosis}

In high-income countries (HICs) routine antenatal scans may detect more than $97 \%$ of cases [23]. A diagnosis can be made as early as 10 weeks of gestation [24] and aids counseling, transfer and delivery $[25,26]$. Ultrasound will typically pick up herniated bowel not covered by amnion, to the right of the umbilical cord (Figure 2). In contrast, an exomphalos will be covered by a membrane, lies in the

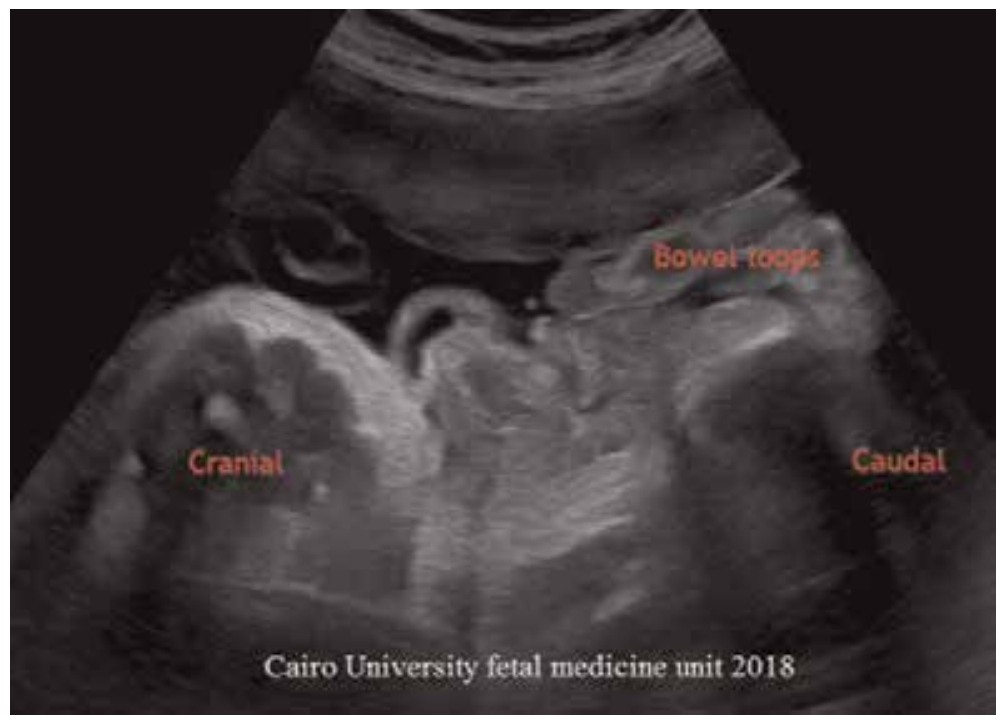

Figure 2.

Antenatal scan showing GS. 
midline and may involve solid organ prolapse. Ultrasound is instrumental in picking up closing GS which is defined as a worsening ratio of intra vs. extra peritoneal bowel dilatation [27]. Further aids to diagnosis of GS are high levels of maternal serum alpha-fetoprotein (MSAFP) [28], intrauterine growth retardation with or without oligo-/an-hydramnios $[7,29,30]$. As GS is usually an isolated anomaly with very few risks to the mother or child, termination of pregnancy is not habitually offered $[2,31,32]$.

\section{Timing and mode of delivery}

A spontaneous onset of labor will typically occur around 36 weeks gestation and the route of delivery is dependent on obstetric indications $[33,34]$. There is a lack of high-level evidence to support early induction of labor in uncomplicated GS cases $[35,36]$ and a similar lack of evidence to support cesarean section [37]. Early (emergency) delivery is beneficial in closing GS [26].

\section{GS in low to middle income countries (LMICs)}

LMICs have an overall high mortality rate in neonates with correctable congenital anomalies $[38,39]$ and suffer from a lack of medical facilities and personnel [40]. Non-governmental and governmental organizations have been criticized for not doing enough $[41,42]$ though new partnerships are attempting to redress this $[43,44]$.

Mortality from GS in low-to-middle-income countries (LMICs) can reach up to 80-100\% [45-48] which is in sharp contrast to the $<10 \%$ in HICs [49]. Sepsis is a major culprit in most cases of neonatal mortality in LMICs [48]. The Gastroschisis International (GiT) network has suggested that poor resuscitation combined with sepsis and abdominal compartment syndrome is directly linked to the poor outcome [50].

Antenatal care may not be well developed [51] or mothers may engage poorly with it [52] which risks births in areas far from the reach of the pediatric surgeon. A delay in transfer of the neonate with GS remains a main concern $[47,53]$ however a recent study from South Africa has suggested that resuscitation at the initial point of care and throughout transfer may be the key to improving the end result [51].

\section{Initial management (pre-operative management)}

A GS infant is ideally delivered at or near a facility with pediatric surgical support [25]. Conversely, outborn cases have been shown to have worse outcomes such as longer days on parenteral nutrition and longer duration to achieving full feeds [54].

The accepted approach to managing GS is to cover the gut with a sterile bag (Figure 3), nasogastric decompression and fluid resuscitation. Hypothermia is a major risk due to the exposed gut and significant fluid losses [55]. Premature babies are particularly prone to hypothermia because of their high ratio of skin surface to weight and a lower amount of subcutaneous and brown fat. They may also have respiratory issues which impact on their oxygen consumption and heat production [55].

The authors follow the protocol outlined in Figure 4. At the outset doctors and nurses are reminded that the triad of hypovolemia, hypothermia and sepsis are the major threats to this neonate and that resuscitation is directed to mitigating their 


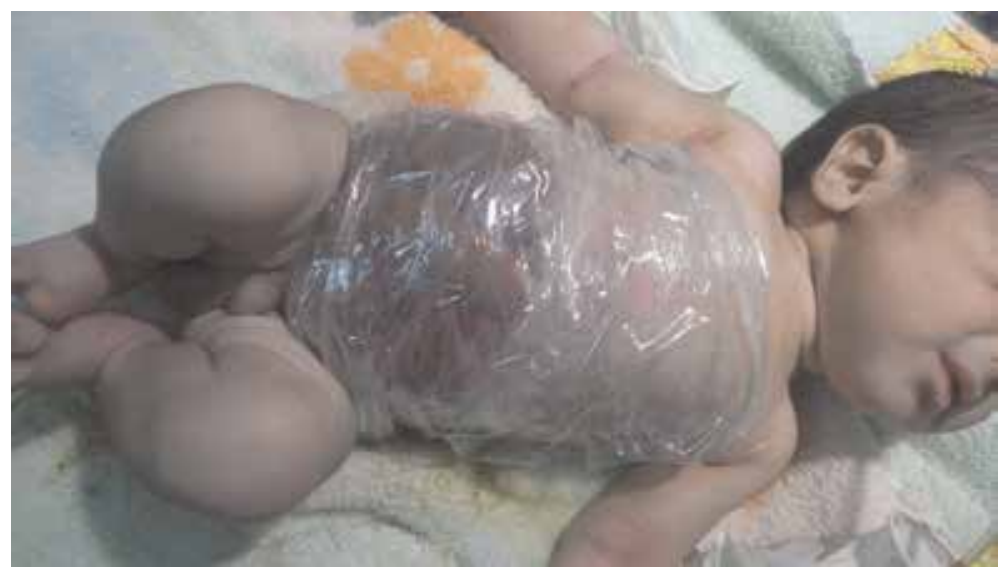

Figure 3.

Cling film covering the bowel in gastroschisis.

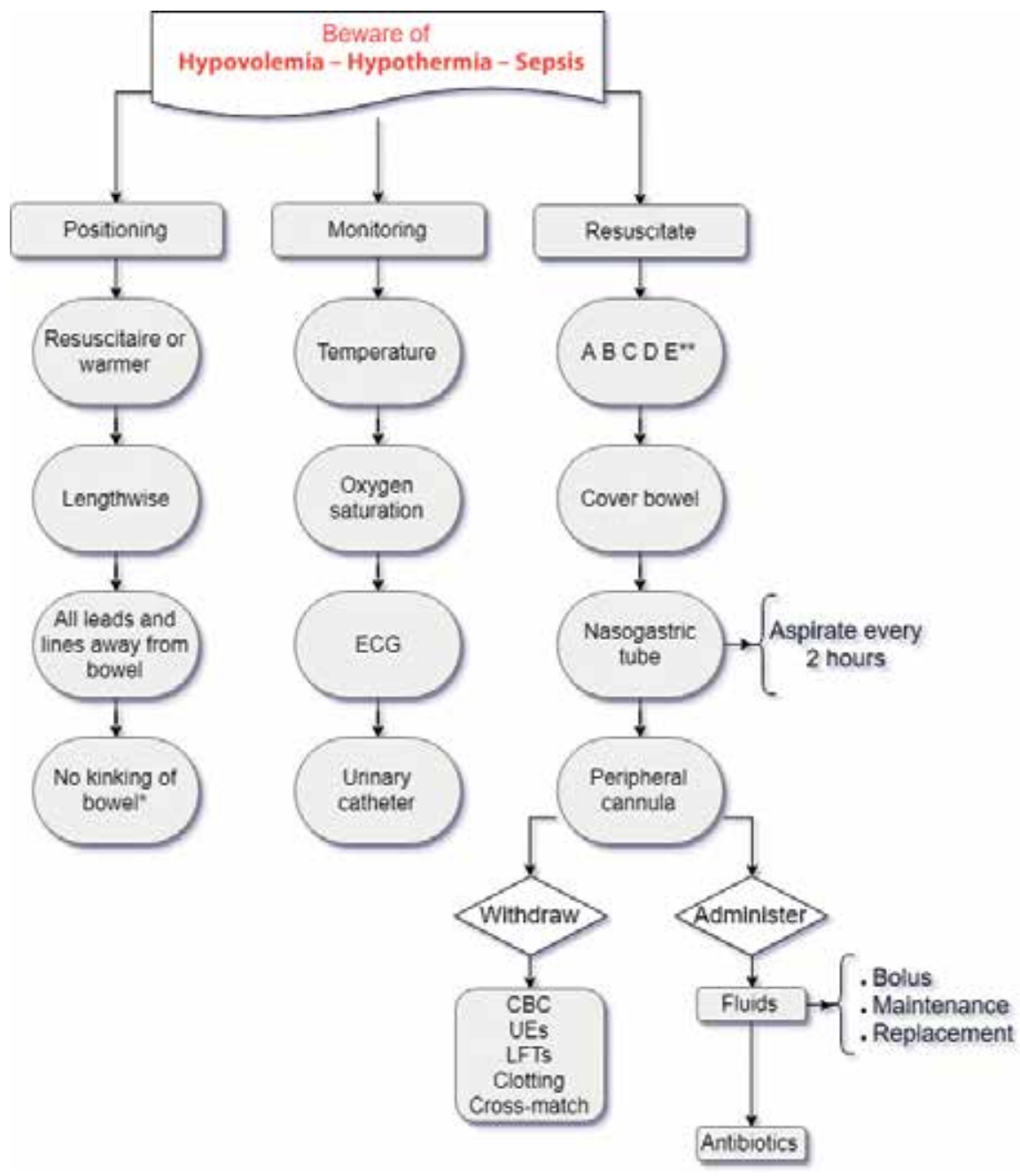

Figure 4 .

Initial management of gastroschisis. "Kinking can be avoided by laying the child on their side or by propping up the bowel with gauze rolls while the child is supine. "ABC of basic resuscitation. Do not forget blood sugar. $C B C$, complete blood count; UEs, urea and electrolytes; LFTs, liver function tests. 
effects. Almost simultaneously, certainly not sequentially, the baby is positioned lengthwise on a resuscitaire or warmer to facilitate access. Any wires, leads or lines are shifted away from the baby and the bowel. Kinking of the bowel is avoided by laying the child on their side or by propping up the bowel with gauze rolls in the supine position. Probes for temperature and oxygen saturation are connected. ECG leads are placed and connected to a monitor. A urinary catheter is placed with an aseptic technique. Resuscitation follows APLS guidelines of airway, breathing, circulation, rapid initial examination while the bowel is covered with cling film. An appropriately-sized nasogastric tube is placed on free drainage supplemented by 2-hourly active aspiration. Peripheral vascular access is secured and bloods are taken for blood sugar (if not done earlier), a complete blood picture, kidney and liver functions, clotting and cross-match. A fluid bolus is then administered followed by maintenance according to body weight. Broad-spectrum antibiotics are given according to the hospital protocol.

\section{Intra-operative decision making}

The aspired aim is to achieve full reduction of the bowel with muscle and skin closure of the abdominal wall, as cosmetically as possible. Safety of the child and the gut are paramount therefore if a complete primary closure is not possible staged reduction should be considered.

All manipulations should be done in a sterile environment. The authors routinely take all cases to theatre, however bedside procedures are also possible. Central vascular access is secured and a urinary catheter would have been placed during initial resuscitation in all cases.

The authors follow the guideline outlined in Figure 5: cases of simple GS with no obvious viscero-peritoneal disproportion (VPD) will undergo primary closure. If very straightforward, sutureless closure with steri-strip dressings is done. On occasion some cases will require division of bands or strands of omentum adherent to the defect and they go on to have formal sutured closure of the defect. Primary (sutured) closure has excellent cosmetic results (Figures 6 and 7). Sutureless closure is associated with a higher incidence of umbilical hernia $[56,57]$ Guided by ventilation pressures, cases with moderate VPD will undergo a skin closure with the size of the defect determining if the umbilicus, the skin or a prosthetic patch is needed. Marked VPD and high ventilation pressures call for staged silo closure. The authors fashion surgical silos from sterile intravenous fluid bags (Figure 8a-c). Surgical silos can be made from a variety of materials which are summarized in Box 1. Spring-loaded (pre-formed) silos are ready-made and obviate the need for suturing to the abdominal wall $[20,55]$. They come in various sizes to allow for the variability in the GS defect (Figure 9). One may rely on gravity alone, active tucking or a combination of both to reduce the contents into the abdominal cavity. There is weak evidence in favor of the routine use of pre-formed silos instead of primary closure $[20,55,58]$.

Complex GS is defined as any case with associated bowel atresia, stenosis, perforation or volvulus. In the presence of atresia, the authors' preference is to plan a delayed repair but a primary resection and anastomosis at the time of abdominal closure is also acceptable if the bowel is healthy and not too edematous. Stoma formation is fraught with high-output stoma complications such as failure to thrive and peri-stoma skin breakdown-therefore is not the surgery of choice in lowresource settings. Closing GS represents a spectrum of disease where the defect has started to narrow down around the prolapsed gut. At its simplest form it can lead to intestinal stenosis but may progress to atresia, gut ischemia up to complete 


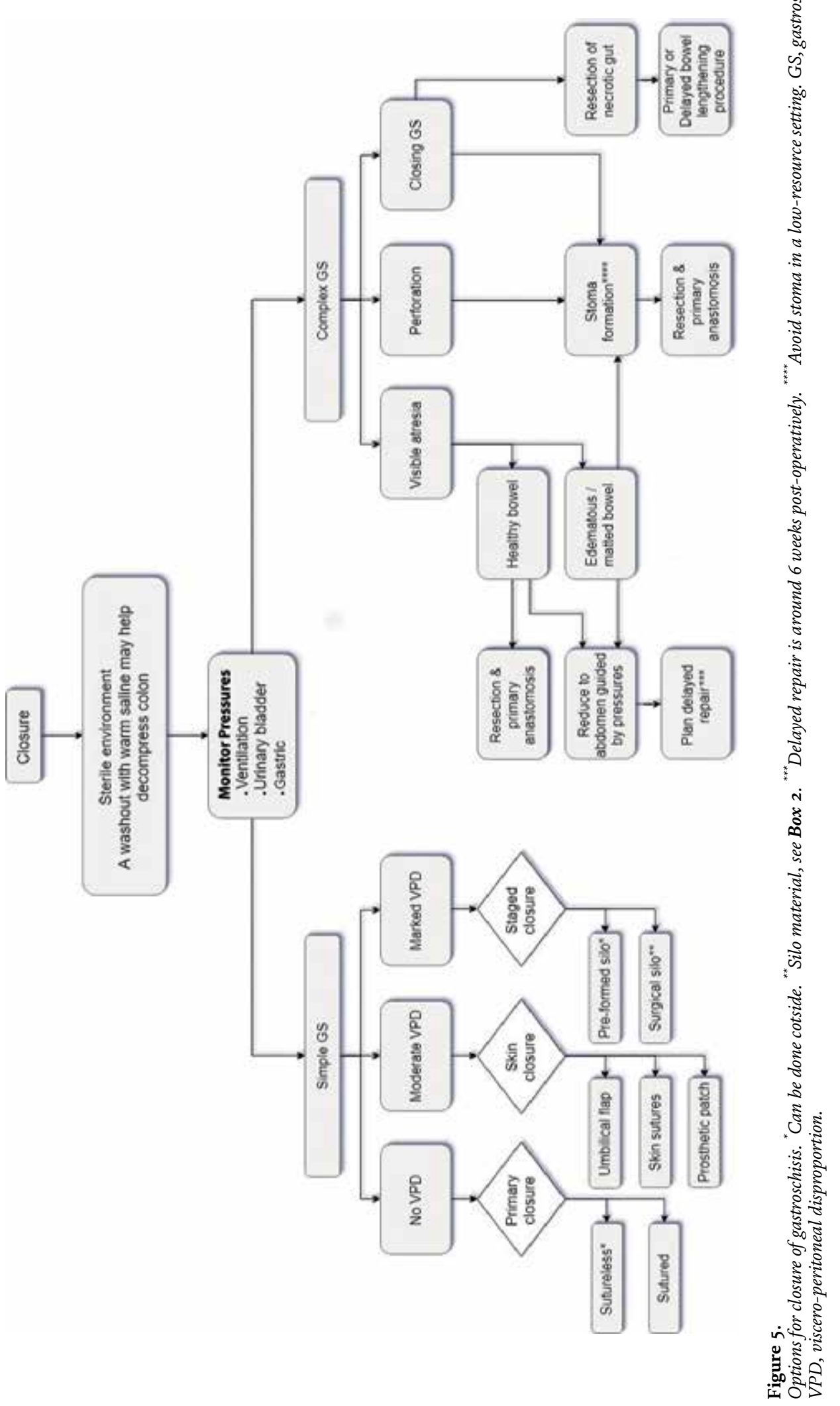




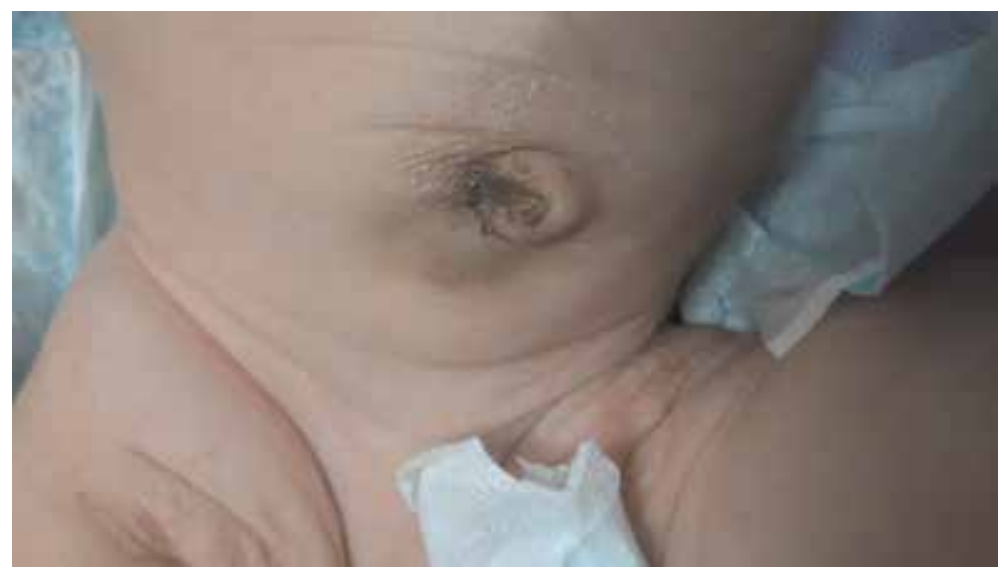

Figure 6.

Cosmetic result after primary closure.

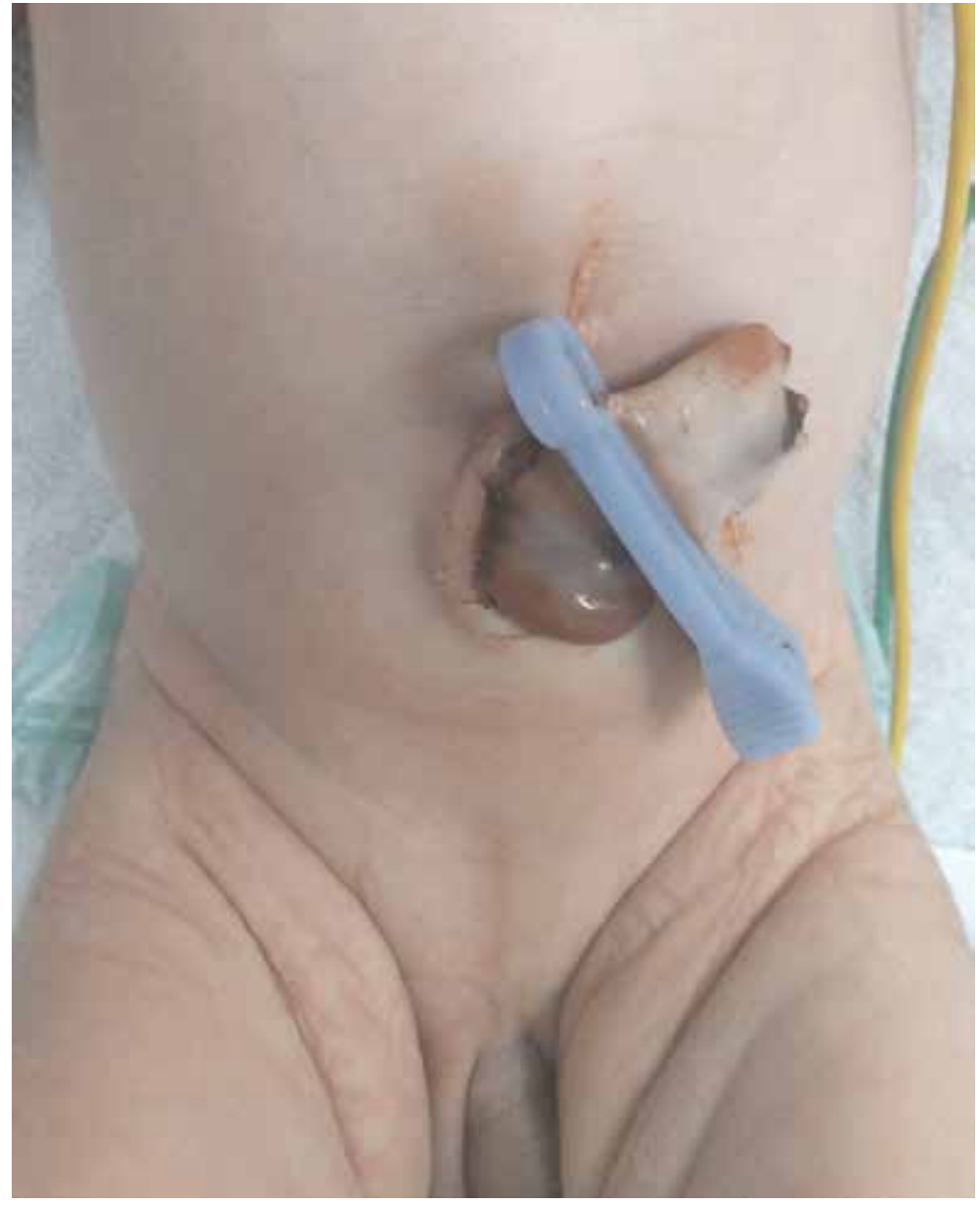

Figure 7.

Primary Closure.

disappearance of the prolapsed bowel if the defect closes completely, aka closed GS or "vanishing gut syndrome" [61]. Closing GS is challenging even in HICs and is associated with worse outcomes compared to simple GS. Narrowing or atresia may 


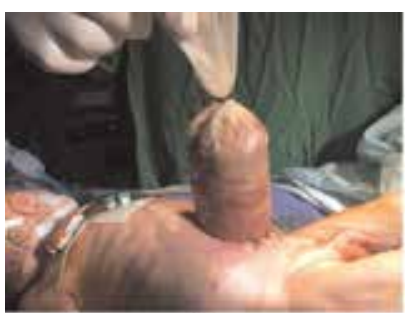

(a)

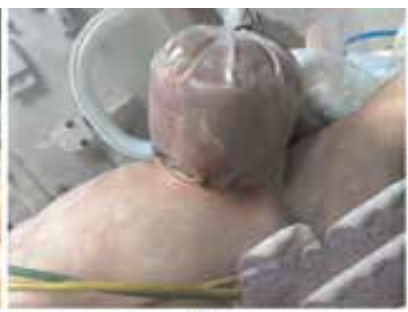

(b)

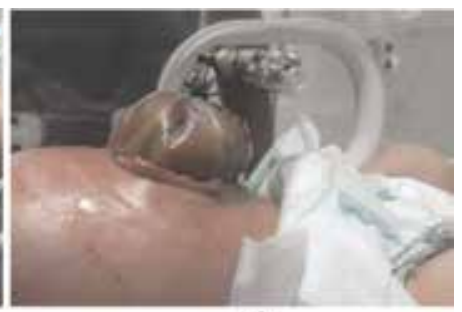

(c)

Figure 8.

$(a-c)$ Staged silo reduction.

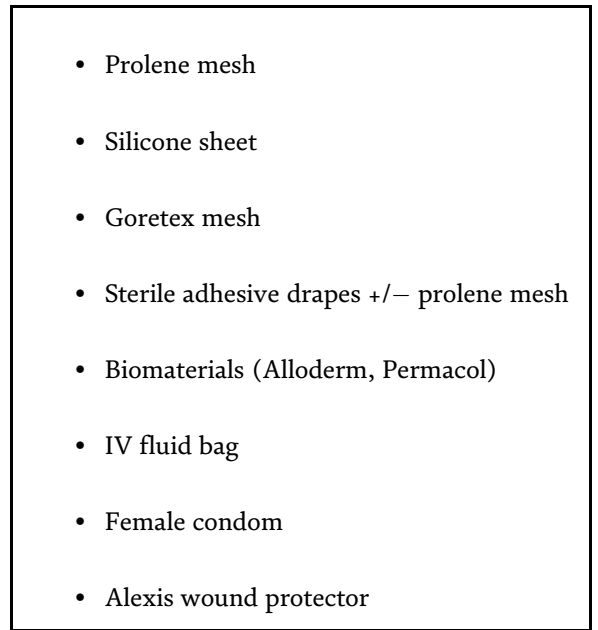

Box 1.

Available silo materials [55, 59, 60].

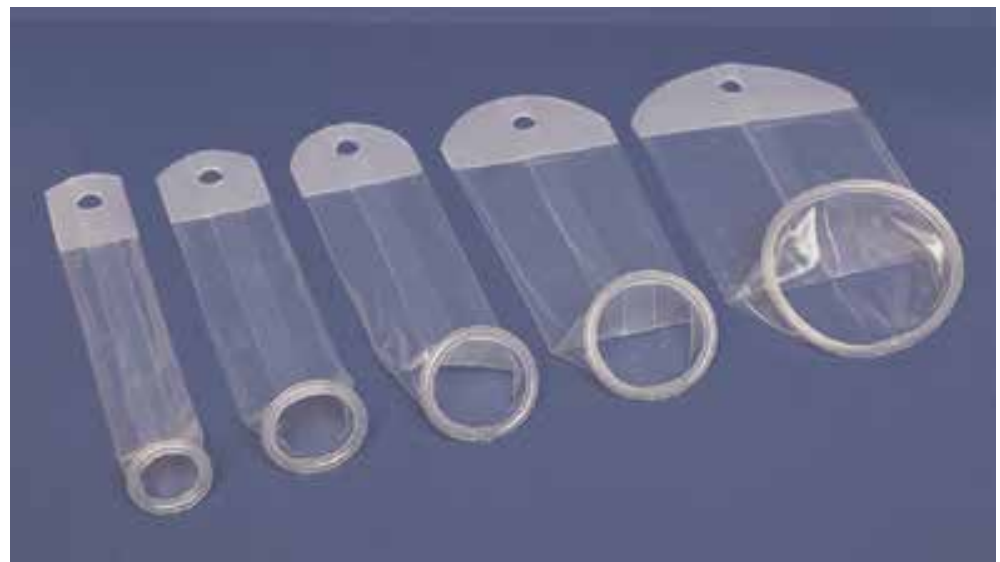

Figure 9.

Pre-formed silos http://bentecmed.com/bentec-medical-products/ventral-wall-defect-silo-bags/.

lend themselves to resection and primary anastomosis (either at the time of reduction, or delayed). Necrotic gut will require resection (Figures 10 and 11) and vanished gut will indicate an ultra-short intra-abdominal segment. These cases will require either primary or delayed bowel lengthening procedures [62]. 


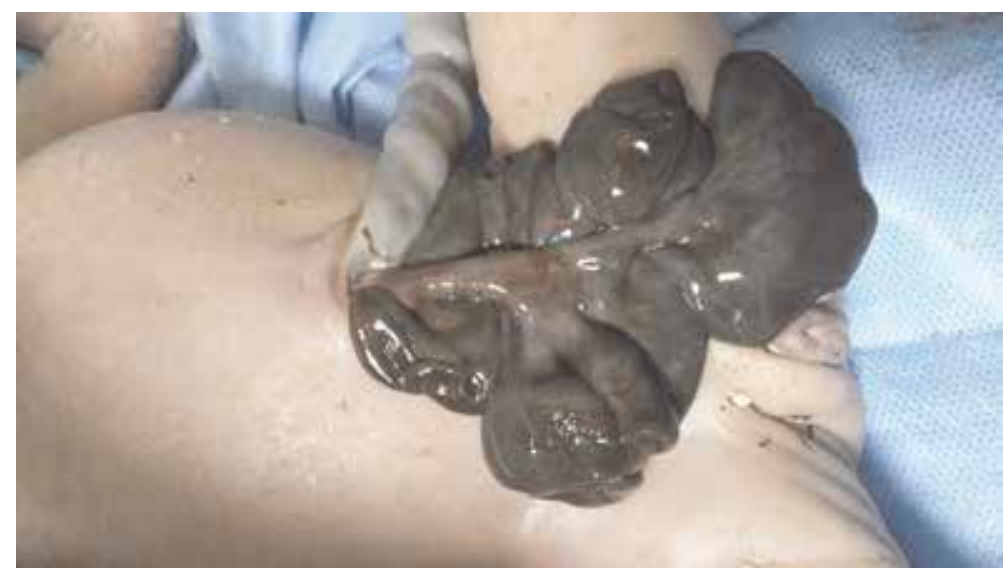

Figure 10.

Closing gastroschisis with necrotic bowel.

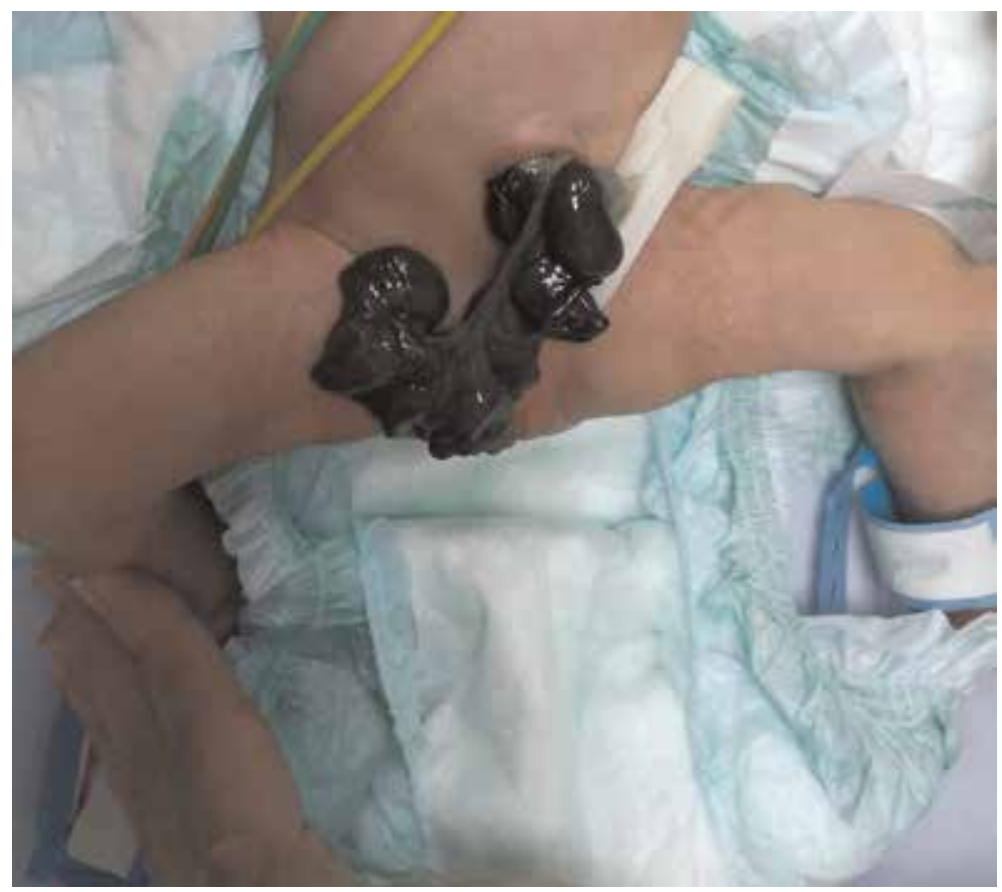

Figure 11.

Closing gastroschisis with necrotic bowel.

\section{Post-operative care}

The staged reduction process should take between 1 day and 2 weeks and is dependent on the degree of VPD. Enteral feeds are started once the gastro-intestinal system shows signs of resumed function: decreased nasogastric aspirates $<20 \mathrm{ml} / \mathrm{kg}$ and bowel motions. Ideally expressed maternal breast milk is used [63-65], but formula feeds are acceptable. Elemental feeds may help protect against necrotizing enterocolitis. GS infants fed at around 7 days post closure seem to have the best outcome [66]. If the bowels do not open within 10-14 days a water-soluble contrast 
enema should be done to rule out a bowel atresia. An atresia detected at the time of initial closure of by subsequent imaging may be safely repaired after 3-6 weeks. Albeit uncommon in GS, cases with any associated malformations will require further investigations and management according to the findings.

\section{Fluid management in GS}

Publications frequently refer to a "consensus" among doctors on the optimal fluids required for GS. However, there is sparse evidence-based literature to guide the perioperative fluid management $[65,66]$. Reports of fluid administration have varied from twice to three-times the normal maintenance volumes for neonates $[67,68]$ and were based on original research by Phillippart et al. in 1972 [69].

Fluid overload in the absence of hypovolemia has been proven to be deleterious in neonates [70]. It affects a patent ductus arteriosus, may cause intracranial hemorrhage, bronchopulmonary dysplasia or may even be fatal [71, 72]. Therefore the mere assumption that GS cases need vigorous volume expansion may be harmful. Preterm neonates may also benefit from fluid restriction according to a recent Cochrane review [73]. It has been suggested that the fluid overload will contribute to intestinal edema leading to a longer hospital stay and longer duration of parenteral nutrition through the increase of total body water and salt [65]. It may also play a part in development of NEC [74].

This practice of over transfusion is routinely carried out postoperatively as well [75]. While it may be of value in cases with a silo where there are ongoing losses of fluid from the base, it has no real justification in cases which undergo primary closure.

Few published sources will give an outright volume to go by. They will always be ranges and the clinician must be guided by continuous assessment of the child. Postnatal diuresis can complicate fluid-balance calculations but a useful milestone to assess the cardiovascular status is after administering $40 \mathrm{ml} / \mathrm{kg}$ of fluid. Albumin has been advocated as a volume expander in hypovolemic GS cases. It is not particularly useful in hypoalbuminemia associated with sepsis [76, 77]. An additional tool to help restore insensible water loss incurred through breathing is the humidification of incubator air.

\section{Complications}

\subsection{Abdominal compartment syndrome (ACS)}

A large degree of viscero-peritoneal disproportion and over-zealous reduction runs the risk of increased intra-abdominal pressure. The latter will result in restricting diaphragmatic movement and compression of the inferior vena cava, which will in turn result in respiratory distress, renal, liver and bowel ischemia, respectively. They manifest as metabolic acidosis, oliguria, renal and liver dysfunction [55].

Frequent monitoring of oxygen saturation/ventilation setting, serial blood gases, urine output, serial abdominal examinations, lower limb perfusion are important in early detection of ACS. Oliguria alone is not a sensitive indicator of ACS as it may be due to hypovolemia. Pressure measurements can be taken using sophisticated transducers used with anesthesia machines or by simply connecting the tubing to a CVP water manometer. Reference values are quoted in Box 2 [78-81]. 
- Gastric/urinary bladder pressure $<200 \mathrm{~cm} \mathrm{H}_{2} \mathrm{O}$ or $<15-$ $20 \mathrm{mmHg}$

- End-tidal $\mathrm{CO}_{2}<50 \mathrm{mmHg}$

- CVP $<4 \mathrm{mmHg}$ or $<5.4 \mathrm{~cm} \mathrm{H}_{2} \mathrm{O}$

- Ventilation $<24 \mathrm{~cm} \mathrm{H}_{2} \mathrm{O}$

Box 2.

Reference values for safe abdominal closure [78-81].

\subsection{Sepsis}

Sepsis is a common complication in LMICs. Most common sources are intra-abdominal, silo/wound infections, indwelling central lines or urinary catheters [50, 51].

As always, prevention is better than cure. Meticulous antisepsis protocols and timely use of antibiotics are important first tools. Early suspicion of central lineassociated blood stream infection (CLABSI) or urinary tract infection (UTI) should prompt urgent cultures to be sent to the lab. The authors remove the urinary catheter once there is a stable urine output and no further risk of abdominal compartment syndrome.

\subsection{Silo complications}

Both pre-formed and surgical silos are prone to dislodgement and may cause bowel kinking, ischemia and perforation [58]. It is prudent to keep the silo and its contents visible at all times to allow early detection of any of these complications.

\subsection{Pneumothorax}

Iatrogenic pneumothorax secondary to barotrauma is an unfortunate complication in ventilated neonates and occurs in around $8.7 \%$ of the cases [82]. In GS this may be due to high intra-abdominal pressure after bowel reduction. This is best anticipated in theatre and if pressures exceed $24 \mathrm{~cm} \mathrm{H}_{2} \mathrm{O}$, a staged reduction should

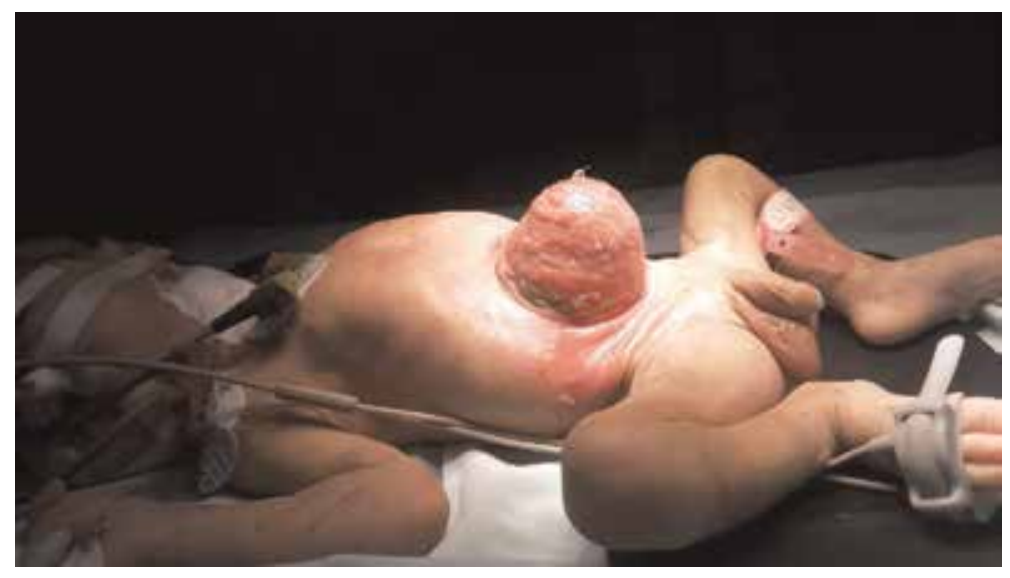

Figure 12.

Large abdominal wall defect with granulation in long-standing silo. 


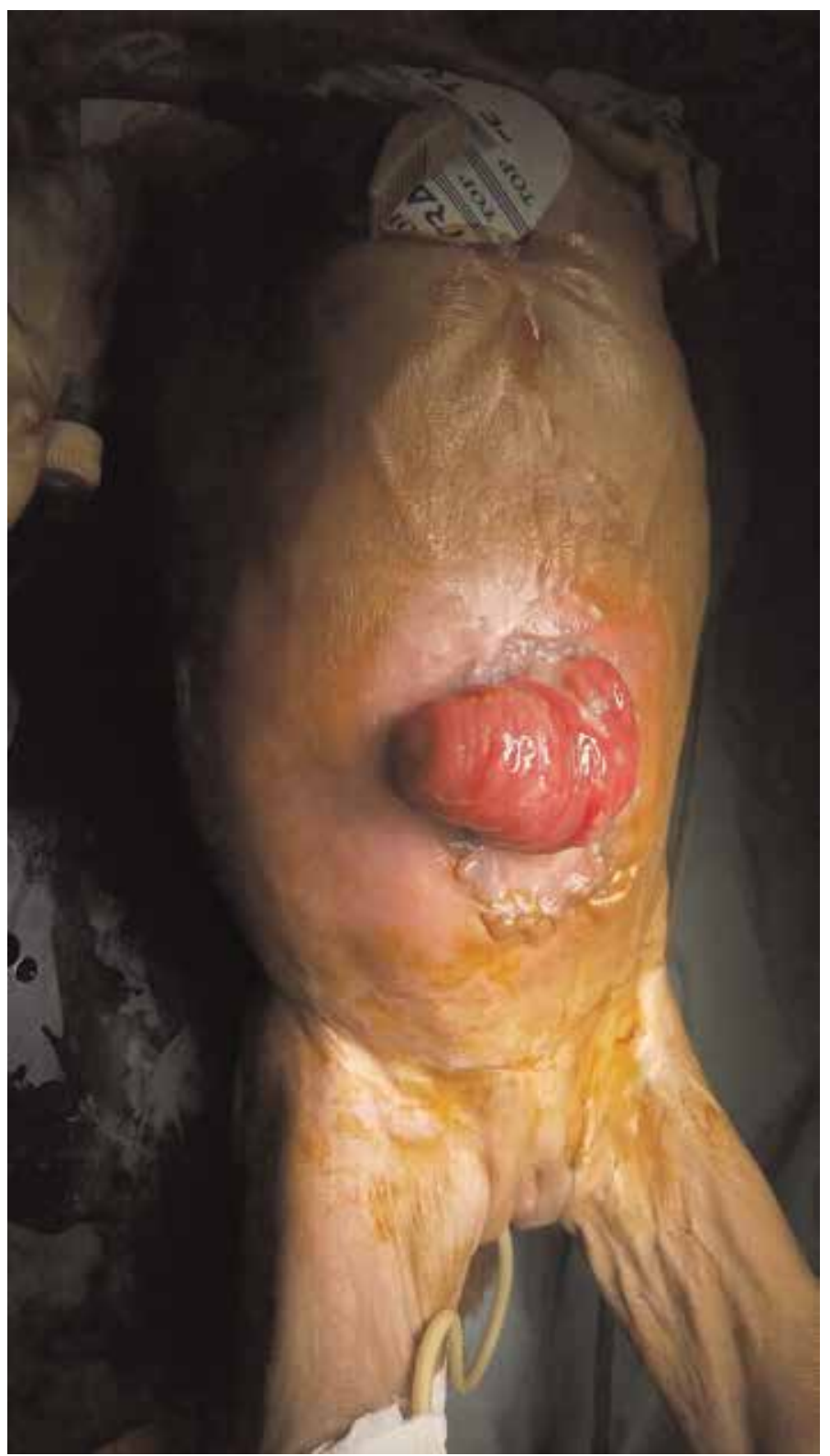

Figure 13.

Entero-cutaneous fistula.

be the surgery of choice. If post-operative ventilation is unavoidable then positive end-expiratory pressure (PEEP) or high-frequency oscillation ventilation (HFOV) is used. Neuromuscular paralysis may also help reduce ventilation pressures but is not always available in low-resource settings.

A pneumothorax is suspected when oxygen saturation drops and ventilation pressures rise sharply with absent ipsilateral air entry. An urgent plain chest x-ray will confirm this and should be followed by immediate needle decompression then a formal chest drain with an underwater seal. Bilateral asynchronous pneumothoraces are not uncommon [82]. 


\subsection{Others}

- NEC: It follows the same patterns and risk factors as with non-GS infants. Prematurity, formula feeds, rapid increase in feed volume-have all been implicated. Treatment is standard: nasogastric tube decompression, gut rest and antibiotics will often suffice $[83,84]$.

- Large abdominal defect: The GS defect is seldom large to start with and is occasionally enlarged by the surgeon to facilitate bowel reduction. Hence a large defect is a rare complication which may occur in long-standing cases of staged-reduction (Figure 12). Standard closure techniques include the use of a prosthetic material or plastic surgery techniques such as abdominal wall rotational flaps with or without lateral release incisions [85].

- Enterocutaneous fistula: (Figure 13) rare complication which may occur secondary to wound infection, NEC, or a combination of both. Vacuum dressings have been of value in treating such a complication $[55,86]$. It is the authors' experience that vacuum dressings may paradoxically cause an enterocutaneous fistula if incorrectly placed or if the suction is too vigorous. Surgical closure when the infant is in a positive nitrogen balance is beneficial.

\section{Author details}

Alaa Obeida* and Aly Shalaby

Pediatric Surgery Department, Cairo University, Specialized Pediatric Hospital, Cairo, Egypt

*Address all correspondence to: alaa.obeida@gmail.com

\section{IntechOpen}

(C) 2019 The Author(s). Licensee IntechOpen. This chapter is distributed under the terms of the Creative Commons Attribution License (http://creativecommons.org/licenses/ by/3.0), which permits unrestricted use, distribution, and reproduction in any medium, provided the original work is properly cited. (cc) BY 


\section{References}

[1] Wilson RD, Johnson MP. Congenital abdominal wall defects: An update. Fetal Diagnosis and Therapy. 2004;19(5): 385-398

[2] David AL, Tan A, Curry J. Gastroschisis: Sonographic diagnosis, associations, management and outcome. Prenatal Diagnosis: Published in Affiliation With the International Society for Prenatal Diagnosis. 2008; 28(7):633-644

[3] Gow KW, Bhatia A, Saad DF, Wulkan ML, Heiss KF. Left-sided gastroschisis. The American Surgeon. 2006;72(7):637-640

[4] Tan KH, Kilby MD, Whittle MJ, Beattie BR, Booth IW, Botting BJ. Congenital anterior abdominal wall defects in England and Wales 1987-93: Retrospective analysis of OPCS data. BMJ. 1996;313(7062):903-906

[5] International Clearinghouse for Birth Defects Surveillance and Research (ICBDSR). ICBDSR Annual Report 2005, with data 2003. Rome: ICBDSR Centre; 2005. www.icbd.org

[6] Parker SE, Mai CT, Canfield MA, Rickard R, Wang Y, Meyer RE, et al. Updated national birth prevalence estimates for selected birth defects in the United States, 2004-2006. Birth Defects Research Part A: Clinical and Molecular Teratology. 2010;88(12):1008-1016

[7] Holland AJ, Walker K, Badawi N. Gastroschisis: An update. Pediatric Surgery International. 2010;26(9): 871-878

[8] Bond SJ, Harrison MR, Filly RA, Callen PW, Anderson RA, Golbus MS. Severity of intestinal damage in gastroschisis: Correlation with prenatal sonographic findings. Journal of Pediatric Surgery. 1988;23(6):520-525
[9] Langer JC, Longaker MT, Crombleholme TM, Bond SJ, Finkbeiner WE, Rudolph CA, et al. Etiology of intestinal damage in gastroschisis. I: Effects of amniotic fluid exposure and bowel constriction in a fetal lamb model. Journal of Pediatric Surgery. 1989;24(10):992-997

[10] Loane M, Dolk H, Bradbury I. A EUROCAT Working Group. Increasing prevalence of gastroschisis in Europ. 1980-2002: A phenomenon restricted to younger mothers? Paediatric and Perinatal Epidemiology. 2007;21(4):363-369

[11] Mac Bird T, Robbins JM, Druschel C, Cleves MA, Yang S, Hobbs CA, et al. Demographic and environmental risk factors for gastroschisis and omphalocele in the National Birth Defects Prevention Study. Journal of Pediatric Surgery. 2009;44(8): 1546-1551

[12] Forrester MB, Merz RD. Comparison of trends in gastroschisis and prenatal illicit drug use rates. Journal of Toxicology and Environmental Health, Part A. 2006; 69(13):1253-1259

[13] Torfs CP, Christianson RE, Iovannisci DM, Shaw GM, Lammer EJ. Selected gene polymorphisms and their interaction with maternal smoking, as risk factors for gastroschisis. Birth Defects Research Part A: Clinical and Molecular Teratology. 2006;76(10): 723-730

[14] Hwang PJ, Kousseff BG. Omphalocele and gastroschisis: An 18year review study. Genetics in Medicine. 2004;6(4):232

[15] Feldkamp ML, Carey JC, Sadler TW. Development of gastroschisis: Review of hypotheses, a novel hypothesis, and 
implications for research. American Journal of Medical Genetics Part A. 2007;143(7):639-652

[16] Hoyme HE, Higginbottom MC, Jones KL. The vascular pathogenesis of gastroschisis: Intrauterine interruption of the omphalomesenteric artery. The Journal of Pediatrics. 1981; 98(2):228-231

[17] Devries PA. The pathogenesis of gastroschisis and omphalocele. Journal of Pediatric Surgery. 1980;15(3):245

[18] Shaw A. The myth of gastroschisis. Journal of Pediatric Surgery. 1975;10(2): 235-244

[19] Duhamel B. Embryology of exomphalos and allied malformations. Archives of Disease in Childhood. 1963; 38(198):142

[20] Haddock C, Skarsgard ED. Understanding Gastroschisis and its Clinical Management: Where are We? [Internet]. Expert Review of Gastroenterology \& Hepatology. Vol. 12. Taylor \& Francis; 2018. pp. 405-415

[21] Blechschmidt E. The Stages of Human Development before Birth: An Introduction to Human Embryology. Philadelphia: Saunders; 1961

[22] Ashburn DA, Pranikoff T, Turner CS. Unusual presentations of gastroschisis. The American Surgeon. 2002;68(8):724

[23] Fillingham A, Rankin J. Prevalence, prenatal diagnosis and survival of gastroschisis. Prenatal Diagnosis. 2008; 28(13):1232-1237

[24] Nicholas SS, Stamilio DM, Dicke JM, Gray DL, Macones GA, Odibo AO. Predicting adverse neonatal outcomes in fetuses with abdominal wall defects using prenatal risk factors. American Journal of Obstetrics and Gynecology. 2009;201(4):383-3e1
[25] Murphy FL, Mazlan TA, Tarheen F, Corbally MT, Puri P. Gastroschisis and exomphalos in Ireland 1998-2004. Does antenatal diagnosis impact on outcome? Pediatric Surgery International. 2007; 23(11):1059-1063

[26] Shalaby A, Davenport M. Closed gastroschisis. Pediatric Surgery International. 2011;27(3):335

[27] Vegunta RK, Wallace LJ, Leonardi MR, Gross TL, Renfroe Y, Marshall JS, et al. Perinatal management of gastroschisis: Analysis of a newly established clinical pathway. Journal of Pediatric Surgery. 2005;40(3):528-534

[28] Palomaki GE, Hill LE, Knight GJ, Haddow JE, Carpenter M. Secondtrimester maternal serum alphafetoprotein levels in pregnancies associated with gastroschisis and omphalocele. Obstetrics and Gynecology. 1988;71(6 Pt 1):906-909

[29] Luton D, Guibourdenche J, Vuillard E, Bruner J. Prenatal management of gastroschisis: The place of the amnioexchange procedure. Clinics in Perinatology. 2003;30(3):551-572

[30] Reid KP, Dickinson JE, Doherty DA. The epidemiologic incidence of congenital gastroschisis in Western Australia. American Journal of Obstetrics and Gynecology. 2003; 189(3):764-768

[31] Campbell KH, Copel JA. Gastroschisis. In: Obstetric Imaging: Fetal Diagnosis and Care. Second ed. 2018. pp. 78-84

[32] Hunter A, Soothill P. Gastroschisis -An overview. Prenatal Diagnosis. 2002;22:869-873

[33] Abdel-Latif ME, Bolisetty S, Abeywardana S, Lui K. Mode of delivery and neonatal survival of infants with gastroschisis in Australia and New 
Zealand. Journal of Pediatric Surgery. 2008;43(9):1685-1690

[34] Lausman AY, Langer JC, Tai M, Seaward PG, Windrim RC, Kelly EN, et al. Gastroschisis: What is the average gestational age of spontaneous delivery? Journal of Pediatric Surgery. 2007; 42(11):1816-1821

[35] Cohen-Overbeek TE, Hatzmann TR, Steegers EA, Hop WC, Wladimiroff JW, Tibboel D. The outcome of gastroschisis after a prenatal diagnosis or a diagnosis only at birth: Recommendations for prenatal surveillance. European Journal of Obstetrics \& Gynecology and Reproductive Biology. 2008;139(1): 21-27

[36] Moir CR, Ramsey PS, Ogburn PL, Johnson RV, Ramin KD. A prospective trial of elective preterm delivery for fetal gastroschisis. American Journal of Perinatology. 2004;21(05):289-294

[37] Mozurkewich E, Chilimigras J, Koepke E, Keeton K, King VJ. Indications for induction of labour: $\mathrm{A}$ best-evidence review. BJOG: An International Journal of Obstetrics \& Gynaecology. 2009;116(5):626-636

[38] Murray CJ, Vos T, Lozano R, Naghavi M, Flaxman AD, Michaud C, et al. Disability-adjusted life years (DALYs) for 291 diseases and injuries in 21 regions, 1990-2010: A systematic analysis for the global burden of disease study 2010. The Lancet. 2012; 380(9859):2197-2223

[39] Black RE, Cousens S, Johnson HL, et al. Global, regional, and national causes of child mortality in 2008: A systematic analysis. Lancet. 2010;375: 1969-1987

[40] Rosano A, Botto LD, Botting B, Mastroiacovo P. Infant mortality and congenital anomalies from 1950 to 1994 : An international perspective. Journal of
Epidemiology \& Community Health. 2000;54(9):660-666

[41] Ameh EA, Chirdan LB. Paediatric surgery in the rural setting: Prospect and feasibility. West African Journal of Medicine. 2001;20(1):52-55

[42] Bickler SW, Kyambi J, Rode H. Pediatric surgery in sub-Saharan Africa. Pediatric Surgery International. 2001;17 (5-6):442-447

[43] Badrinath R, Kakembo N, Kisa P, Langer M, Ozgediz D, Sekabira J.

Outcomes and unmet need for neonatal surgery in a resource-limited environment: Estimates of global health disparities from Kampala, Uganda. Journal of Pediatric Surgery. 2014; 49(12):1825-1830

[44] Galukande M, Luboga S, Kijjambu SC. Improving recruitment of surgical trainees and training of surgeons in Uganda. East and Central African Journal of Surgery. 2006;11(1):17-24

[45] Arnold M. Is the incidence of gastroschisis rising in South Africa in accordance with international trends? A retrospective analysis at Pretoria academic and Kalafong hospitals, 1981-2001. South African Journal of Surgery. 2004;42(3):86-88

[46] Ameh EA, Chirdan LB. Ruptured exomphalos and gastroschisis: A retrospective analysis of morbidity and mortality in Nigerian children. Pediatric Surgery International. 2000;16(1-2): 23-25

[47] Vilela PC, de Amorim MM, Falbo GH, Santos LC. Risk factors for adverse outcome of newborns with gastroschisis in a Brazilian hospital. Journal of Pediatric Surgery. 2001;36(4):559-564

[48] Askarpour S, Ostadian N, Javaherizadeh H, Chabi S. Omphalocele, gastroschisis: Epidemiology, survival, and mortality in imam Khomeini 
hospital, Ahvaz-Iran. Polish Journal of Surgery. 2012;84(2):82-85. DOI: https:// doi.org/10.2478/v10035-012-0013-4

[49] Driver CP, Bruce J, Bianchi A, Doig CM, Dickson AP, Bowen J. The contemporary outcome of gastroschisis. Journal of Pediatric Surgery. 2000; 35(12):1719-1723

[50] Ford K, Poenaru D, Moulot O, Tavener K, Bradley S, Bankole R, et al. Gastroschisis: Bellwether for neonatal surgery capacity in low resource settings? Journal of Pediatric Surgery. 2016;51(8):1262-1267

[51] Stevens P, Muller E, Becker P. Gastroschisis in a developing country: Poor resuscitation is a more significant predictor of mortality than postnatal transfer time. South African Journal of Surgery. 2016;54(1):4-9

[52] Abou-Zahr C, Wardlaw T. Antenatal Care in Developing Countries. Promises, Achievements and Missed Opportunities: An Analysis of Trends, Levels and Differentials 1990-2001. Geneva: World Health Organization, UN Children's Fund; 2003

[53] Harrison DS. Factors associated with mortality among neonates presenting with gastroschisis in Zimbabwe. South African Journal of Surgery. 2006;44(4): 157

[54] Kitchanan S, Patole SK, Muller R, Whitehall JS. Neonatal outcome of gastroschisis and exomphalos: A 10-year review. Journal of Paediatrics and Child Health. 2000;36(5):428-430

[55] Marven S, Owen A. Contemporary postnatal surgical management strategies for congenital abdominal wall defects. Seminars in Pediatric Surgery. 2008;17(4):222-235

[56] Witt RG, Zobel M, Padilla B, Lee H, MacKenzie TC, Vu L. Evaluation of clinical outcomes of sutureless vs sutured closure techniques in gastroschisis repair. Journal of the American Medical Association surgery. 2019;154(1):33-39

[57] Tullie LG, Bough GM, Shalaby A, Kiely EM, Curry JI, Pierro A, et al. Umbilical hernia following gastroschisis closure: A common event? Pediatric Surgery International. 2016;32(8): 811-814

[58] Charlesworth P, Akinnola I, Hammerton C, Praveena P, Desai A, Patel S, et al. Preformed silos versus traditional abdominal wall closure in gastroschisis: 163 infants at a single institution. European Journal of Pediatric Surgery. 2014;24(01): 088-093

[59] Bustorff-Silva JM, Schmidt AF, Gonçalves A, Marba S, Sbragia L. The female condom as a temporary silo: A simple and inexpensive tool in the initial management of the newborn with gastroschisis. The Journal of MaternalFetal \& Neonatal Medicine. 2008;21(9): 648-651

[60] Kusafuka J, Yamataka A, Okazaki T, Okawada M, Urao M, Lane GJ, et al. Gastroschisis reduction using "applied Alexis", a wound protector and retractor. Pediatric Surgery International. 2005;21(11):925-927

[61] Perrone EE, Olson J, Golden JM, Besner GE, Gayer CP, Islam S, et al. Closing gastroschisis: The good, the bad, and the not-so ugly. Journal of Pediatric Surgery. 1 Jan 2019;54(1):60-64

[62] Emil S. Surgical strategies in complex gastroschisis. Seminars in Pediatric Surgery. 2018;27(5):309-315

[63] Jayanthi S, Seymour P, Puntis JW, Stringer MD. Necrotizing enterocolitis after gastroschisis repair: A preventable complication?. Journal of Pediatric Surgery. 1 May 1998;33(5):705-707 
[64] Aljahdali A, Mohajerani N, Skarsgard ED. Effect of timing of enteral feeding on outcome in gastroschisis. Journal of Pediatric Surgery. 1 May 2013;48(5):971-976

[65] Jansen LA, Safavi A, Lin Y, MacNab YC, Skarsgard ED. Preclosure fluid resuscitation influences outcome in gastroschisis. American Journal of Perinatology. 2012;29(04):307-312

[66] Gamba P, Midrio P. Abdominal wall defects: Prenatal diagnosis, newborn management, and long-term outcomes. Seminars in Pediatric Surgery. 2014; 23(5):283-290

[67] Ledbetter DJ. Gastroschisis and omphalocele. Surgical Clinics. 2006; 86(2):249-260

[68] Cooney DR. Defects of the abdominal wall. In: O'Neill JA, Rowe MI, Grosfeld JL, et al., editors. Pediatric Surgery. 5th ed. Vol. 1045. St Louis: Mosby; 1998

[69] Philippart AI, Canty TG, Filler RM. Acute fluid volume requirements in infants with anterior abdominal wall defects. Journal of Pediatric Surgery. 1972;7(5):553-558

[70] Ewer AK, Tyler W, Francis A, Drinkall D, Gardosi JO. Excessive volume expansion and neonatal death in preterm infants born at 27-28 weeks gestation. Paediatric and Perinatal Epidemiology. 2003;17(2):180-186

[71] Arikan AA, Zappitelli M, Goldstein SL, Naipaul A, Jefferson LS, Loftis LL. Fluid overload is associated with impaired oxygenation and morbidity in critically ill children. Pediatric Critical Care Medicine. 2012;13(3):253-258

[72] Willson DF, Thomas NJ, Tamburro R, Truemper E, Truwit J, Conaway M, et al. The relationship of fluid administration to outcome in the pediatric calfactant in acute respiratory distress syndrome trial. Pediatric Critical Care Medicine. 2013;14(7): 666-672

[73] Bell EF, Acarregui MJ. Restricted versus liberal water intake for preventing morbidity and mortality in preterm infants. Cochrane Database of Systematic Reviews. 2014;12. Artical ID: CD000503

[74] Oldham KT, Coran AG, Drongowski RA, Baker PJ, Wesley JR, Polley TZ. The development of necrotizing enterocolitis following repair of gastroschisis: A suprisingly high incidence. Journal of Pediatric Surgery. 1988;23(10):945-949

[75] Bonasso PC, Lucke-Wold B, Hobbs GR, Vaughan RA, Shorter NA, Nakayama DK. Excessive postoperative fluid administration in infants with gastroschisis. The American Surgeon. 2016;82(8):704-706

[76] AL-Khafaji A, Webb AR. Should albumin be used to correct hypoalbuminemia in the critically ill? No. Transfusion Alternatives in Transfusion Medicine. 2003;5(4): 392-396

[77] Vincent JL, Russell JA, Jacob M, Martin G, Guidet B, Wernerman J, et al. Albumin administration in the acutely ill: What is new and where next? Critical Care. 2014;18(4):231

[78] Puffinbarger NK, Taylor DV, Tuggle DW, Tunell WP. End-tidal carbon dioxide for monitoring primary closure of gastroschisis. Journal of Pediatric Surgery. 1996;31(2):280-282

[79] Olesevich M, Alexander F, Khan M, Cotman K. Gastroschisis revisited: Role of intraoperative measurement of abdominal pressure. Journal of Pediatric Surgery. 2005;40(5):789-792

[80] Banieghbal B, Gouws M, Davies MR. Respiratory pressure monitoring as 
an indirect method of intra-abdominal pressure measurement in gastroschisis closure. European Journal of Pediatric Surgery. 2006;16(02):79-83

[81] Hong CM, Patel A. Novel intraoperative pulse oximetry monitoring for gastroschisis: A noninvasive monitor of intra-abdominal pressure. Pediatric Anesthesia. 2008;18(4):344-345

[82] Watkinson M, Tiron I. Events before the diagnosis of a pneumothorax in ventilated neonates. Archives of Disease in Childhood-Fetal and Neonatal Edition. 2001;85(3):F201-F203

[83] Snyder CL. Outcome analysis for gastroschisis. Journal of Pediatric Surgery. 1999;34(8):1253-1256

[84] Gupta R, Cabacungan ET. Outcome of neonates with gastroschisis at different gestational ages using a national database. Journal of Pediatric Surgery. 2018;53(4):661-665

[85] Svetanoff WJ, Zendejas B, Demehri FR, Cuenca A, Nath B, Smithers CJ. Giant gastroschisis with complete liver herniation: A case report of two patients. Case Reports in Surgery. 2019; 2019:8. Article ID: 4136214. https://doi. org/10.1155/2019/4136214

[86] Waldhausen JH. Surgical management of gastroschisis. NeoReviews. 2005;6(11):e500-e507 

Section 5

\section{Minimally Invasive Surgery}





\title{
Single-Incision Pediatric Endosurgery (SIPES)
}

\author{
Enaam Raboei, Ameen Alsaggaf, Yazeed Owiwi, \\ Syed Salahuddin, Alaa Ghallab and Mazen Zidan
}

\begin{abstract}
Most centers advocate laparoscopy in order to minimize the size and the number of skin incisions. Many comparative studies, systematic review, and pooled analysis demonstrate that single-incision laparoscopic surgery (SILS) is comparable to conventional laparoscopic surgery (CLS). However, this review identifies the need for randomized controlled trials to clarify the efficacy of SIPS compared with CLS. SIPES pediatric has gained significant popularity. Longer M OT with SIPES was the main concern in most published series. One study has shown that SIPES in children is safe and feasible when performed by resident doctors in comparison to the fellow. We started SIPES in 2003. It is carried out routinely by trainees and specialists. Interferences and collisions between surgical instruments are worse in SIPES than CLS. These challenges extended the OT. Although the use of flexible laparoscopic instruments instead of straight instruments may overcome these technical difficulties, only straight laparoscopic instruments are currently used in our institution. Our aim is to standardize this approach in pediatric age group. The technique can be imparted satisfactorily to trainees. However, its successful incorporation into surgical training programs will depend on the development of innovative simulation strategies.
\end{abstract}

Keywords: SILS, SIPES, single incision, laparoscopy

\section{Introduction}

There are many nomenclatures and abbreviations found in literatures: SILS, single-incision laparoscopic surgery; LESS, laparo-endoscopic single-site surgery; TUES, trans-umbilical endoscopic single-site surgery; SPA, single portal access; E-NOTES, embryologic natural orifice transluminal endoscopic surgery; SAS, single access surgery; S3, single-site surgery; NOTUS, natural orifice transumbilical surgery; SAVES, single access video endoscopic surgery; and SIPES, single-incision pediatric endoscopic surgery. Cultural changes affected the way of managing patients. It has been over 30 years since the first laparoscopic cholecystectomy. Von Ott inspected the abdominal cavity of pregnant women in 1901. Georg Kelling performed "koelioscopie." Jacobeus published his first report "Laparothorakoskopie." Lukichev in 1983 and Muhe in 1985 performed laparoscopic cholecystectomy in humans [1]. Kalloo performed trans-gastric peritoneoscopy in 2004 [2]. Multiple centers performed NOTES in humans, trans-gastric appendectomy and transvaginal cholecystectomy. Limitations of NOTES technique lead to make SILS to go in parallel with it [3]. The first published report in general surgery 
appeared in 1992 with appendectomies [4]. In the same year, D'Alessio described a technique for appendectomy in pediatric patients in which a special port was used at the umbilicus to allow the surgeon to bring the appendix out through the umbilicus to perform an extracorporeal appendectomy [5]. 19\% of 166 patients in their series required additional trocars, and $4 \%$ required conversion to an open operation. The MOT (MOT) was 35 min with a 7-day return to normal activity, compared with 10 days for those that required additional trocars. SIPES has been introduced in our institute by 2003. Now SIPES is our standard technique for many procedures like cholecystectomy, splenectomy, appendectomy, assisted Mitrofanoff, SIPES hernia repair percutaneous internal ring suturing (PIRS), ovarian cystectomy, and fundoplication since 2011. We have performed around 400 SIPES cases up-to-date.

\section{Limitations of SIPES}

SIPES is not a new technique. It is actually a modification of current laparoscopic technique with some modified instruments and approach used successfully for multiple laparoscopic procedures. Almost all cases which could be done by conventional laparoscopy are amenable to be done by SIPES like splenectomy, appendectomy, cholecystectomy, colectomy, anterior resection, hernia repair, splenectomy, Nissen fundoplication, and sleeve gastrectomy. The approach failed to gain momentum for several years, due to technical limitations with conventional instrumentation. These limitations did not prevent surgeons from using SIPES in pediatric age group. Barbaros and Dinccag [6] published the first two adult cases of SILS splenectomy in 2009. Later Dutta reported the first SIPES splenectomy in 2012 [7]. Recently still few centers worldwide have advanced pediatric SIPES [7-13].

\section{Advantage of SIPES}

Fewer incisions, cosmesis, and non-violation of natural orifices are the most attractive reasons for the patient to choose SILS technique; as a surgeon, ease of tissue retrieval and combination procedures are the main reasons. Using standard laparoscopic equipment has facilitate the procedure for the surgeon with less cost.

\section{Disadvantage of SIPES}

In comparison with CLS, increased postoperative pain and violation of ergonomic principles in SIPES were not proven in the published studies. Hernia might be a problem in adults published series, but we did not encounter any in our patients. In reverse SIPES, it is an opportunity to repair the umbilical hernia when closing the wound in pediatric age group. Wound infection was not different from conventional laparoscopy. Learning curve escalates fast, and the cost-effectiveness was proven to be less costly than conventional laparoscopy [3].

\section{Public opinion}

Which technique the public choose if NOTES and SIPES were the only options offered to them? [14]

Most people choose SILS over NOTES when asked about this scenario. 
- $208(20.7 \%)$ choose NOTES

- 795 (79.0\%) choose SILS

- $3(0.3 \%)$ would refuse surgery

1006 individuals completed the questionnaire explained by F1 medical intern, so as not to bias anyone. All individuals were from around London. 458 (45.5\%) were males and 548 (54.5\%) were females. 129 were < 20 yrs. old, 460 were 20-29 yrs. old, 186 were 30-39 yrs. old, 103 were $40-49$ yrs. old, 61 were 50-59 yrs. old, and 67 were $\geq 60$ yrs. old. 80 were physician, 88 medical students, 39 were nurses, and 6 were NHS admin [15]. 793 were nonmedical. Taking the choices by gender, profession, and age, the preference order for the four approaches was:

- 1st-SILS

- 2nd-conventional lap

- 3rd-NOTES

- 4th-open

Summary

- SIPES is safe and feasible technique for pediatric age group.

- The disadvantages are balanced with the patient satisfaction and excellent cosmetic results.

\section{Port position}

The position of SIPES port may be different depending on many factors like choice of surgeon, type of surgery, and the age of the patient. The different locations commonly used are:

1. Omega-shaped incision made around the upper half of umbilicus.

2. Trans-umbilical incision.

3. Incisions are also given above and below umbilicus.

4. Incision maybe placed in the pubic hairline medially.

The author prefers trans-umbilical incision as its ideal to prevent any visible scar, easy access, and quick closure with no port site hernia. In pediatric it gives opportunity to repair the umbilical hernia.

\section{Types of ports}

There are many types of ports with different sizes in the market. The devices are either disposable or reusable: SILS device by Covidien (C) (Medtronic), GelPOINT system by Applied Medical, R-Port and TriPort by Advanced Surgical Concepts, 


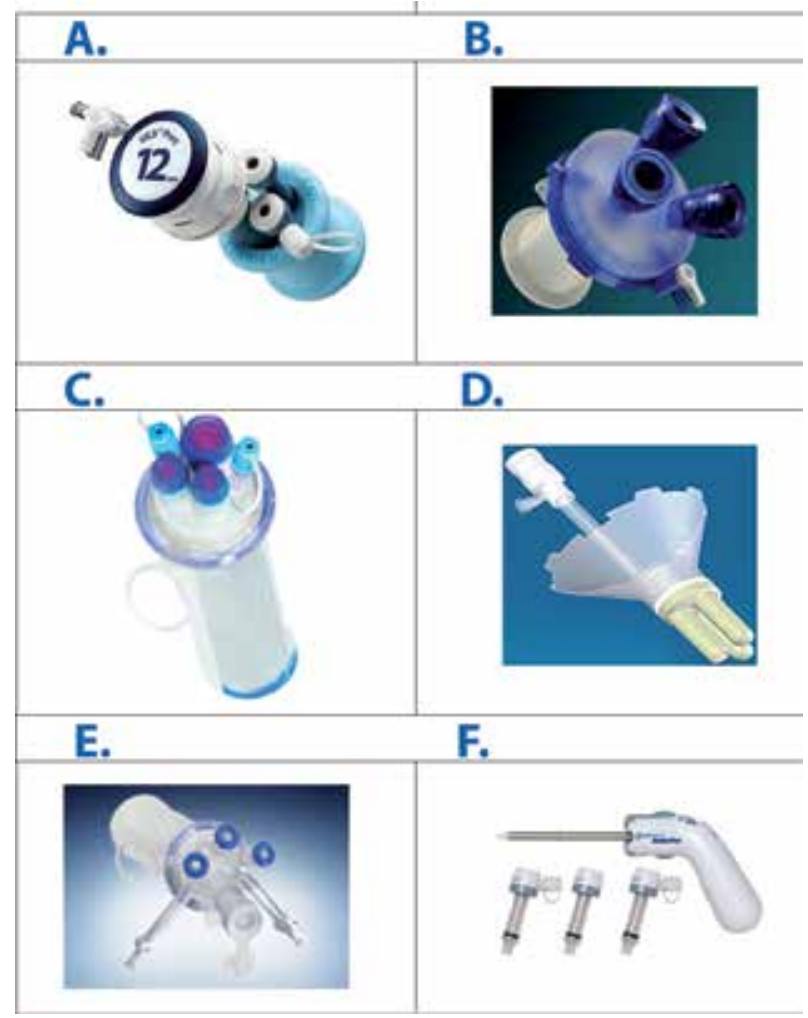

Figure 1.

Types of ports. A) Sils covidien, B) Gel point, C) Triport, D) Uni-X, E) Quadric port. F) Anchorport R by Surgiquest.

Uni-X by Pnavel, Tri or quadric port by Olympus, and AnchorPort R by SurgiQuest. (Figure 1) Each port has its own unique feature, so the selection of the port depends on the surgeon, availability, and cost factor. The author has found that Covidien (C) (Medtronic) port is the most suitable one as it is small in size and it can adopt $15 \mathrm{~mm}$ port for introducing GIA stapler and endobag.

\section{Problems and solutions seen in SIPES}

Rivas [16] has given a good list of problems and solutions in SILS. The encountered problems were clashing of instruments, lack of ideal operative ports, interference and deflection of laparoscope's light source by operating instruments, interference of wires or tubing that connect perpendicularly to instruments, difficulty with retraction of organs or structures, lack of time and patience to learn, loss of proprioception due to crossed instrument, and change of surgeon's mindset. The solutions suggested by him were the use of curved, reticulating, or flexible instruments, use of novel multichannel ports, use of an extra-long 5-mm angled laparoscope $(50 \mathrm{~cm})$, use of retracting sutures, continuous medical education, implementation of magnetically anchored instruments deployed though a single incision, and use of a $90^{\circ}$ adaptor for the light source (for sharp change in its direction parallel to the laparoscope).

Collision of instruments is considered the main limiting factor for popularizing this technique. Other surgeons conquer this problem by using articulating instruments and angled tip fiber-optic camera. The use of articulating instruments was 
difficult and almost impossible in small age group due to narrow and small working space, so the author has replaced it by standard straight instruments. The cameraman can stand away from the operating surgeon by using a $50 \mathrm{~cm}$ long laparoscope. The articulating and bent instrumentation requires training and experience, and we as other SIPES surgeons are confident that they are not needed in neonates and children. Bent graspers and the $45^{\circ}$ rigid endoscope or the deflectable tip laparoscope to minimize instrument collision, both internally and externally, might be of help in obese children and bariatric surgery.

Retraction difficulties is again one of the most important challenges in SIPES; to overcome this problem, there are many tricks being used by surgeons like placing a thin grasper (2-mm Minilap Alligator-Stryker Endoscopy, San Jose, CA), transabdominal sutures, insertion of additional trocarless instrument, and using special laparoscopic magnetic graspers which coupled with external magnets $[16,17]$. Transabdominal suturing is useful in small children due to their thin abdominal wall. These sutures are used to encircle the round ligament for liver retraction and seromuscular bites through hollow organs like intestine and gallbladder $[16,19]$.

Coordination between the surgeon and the camera driver is essential as much or more than it's needed in CLS. In case of intraoperative complication or failure of progression, the introduction of other transabdominal conventional laparoscopic ports to aid completion of the surgical procedure is still there.

Engagement of OR staffs is essential to develop SIPES skills. In the beginning "converting an easy procedure into a harder one" is the sentence which you will hear as a SILS surgeon. Surgeons performing SIPES should have MIS skills and should attend SILS workshops.

In our institute we are providing surgical trainees with advanced laparoscopic and SILS animal workshops. The trainees are doing many SIPES procedures during their rotation, like appendectomies, cholecystectomies, and splenectomies. Although our rotating trainees did not have previous experience in SILS, as we are the only SILS center in the region, by the end of their rotation, they will be able to perform SIPES safely.

\section{Summary}

- The position of SIPES port is mainly trans-umbilical, but it might be introduced in the left upper quadrant for sleeve gastrectomy.

- Many types of ports are available, but Covidien (c (Medtronic) is more suitable for pediatric patients, and Olympus is more suitable for bariatric surgery.

- Many challenges exist with SIPES, but there are always solutions as in conventional laparoscopy.

- Surgeons performing SIPES should have MIS skills and should attend SILS workshops.

- Insertion of extra port is always an option and is considered as conversion to mini laparoscopy (two ports).

\section{SIPES tips and tricks}

\subsection{Tips \& Tricks in SIPES appendectomy}

SIPES appendectomy is increasing for its well-known cosmetic benefit. SIPES appendectomy is the most common procedure done in pediatric surgical units. The incision is strategically placed in the umbilicus with a perceived scarless abdomen. The technique is almost the same as in conventional procedure; it is performed 
through a $1.5 \mathrm{~cm}$ umbilical incision. Single-incision port Medtronic $5-12 \mathrm{~mm}$ is placed using the open technique. The mesoappendix is divided by diathermy or sealing device, endo-loops is applied to secure the base of the appendix, and the appendix is then divided and retrieved through the port. Interferences and collisions between surgical instruments are worse than they are when conventional laparoscopic appendectomy (CLA) is performed using three incisions; this may extend the MOT. However, even with these challenges, difficult appendectomy can be completed successfully and safely by SIPES.

\subsection{SIPES cholecystectomy}

Cholecystectomy is one of the most popular SIPES procedures. Our technique is to place a SILS port Covidien (C) (Medtronic) 5-12 mm in $1.5 \mathrm{~cm}$ trans-umbilical incision by open access. Obtaining the critical view of safety to properly visualize the cystic duct and artery is perhaps of utmost importance. The author modified the placement of straight needle for gallbladder fundus traction by transabdominal suture which is introduced percutaneously by curved needle. Once the gallbladder is properly retracted, the cystic duct and artery are identified, double clipped, and divided. The gallbladder is then dissected off the liver bed with hook cautery, and when completely detached, it is extracted from the abdomen through $12 \mathrm{~mm}$ port. No need for endobag.

\subsection{SIPES splenectomy}

SIPES splenectomy gives a good access for retrieving large-sized spleen through the umbilicus, instead of Pfannenstiel-Kerr incision used in conventional laparoscopic splenectomy CLS. Our technique is to place the patient in supine position with left side tilted $30^{\circ}$. Open-access trans-umbilical single-incision of $1.5 \mathrm{~cm}$ is used for placement of SILS port, Covidien (Medtronic) 12-15 mm SILS ports. Pneumoperitoneum is created and maintained at a constant pressure of 10-12 mm Hg. We insert $3 \mathrm{~mm}$ extra port in left flank for splenic retraction. We use only straight regular instruments. Dissection is performed by sealing device (LigaSure) in four stages: division of spleno-colic ligament at lower pole, dissection of vascular hilum, division of short gastric vessels, and detachment of diaphragmatic ligaments. The spleen is placed in a plastic bag and retrieved after morcellation with Péan forceps.

Insertion of portless extra $3 \mathrm{~mm}$ port in the left upper quadrant is necessary for elevation of the spleen to facilitate dissection of the hilum [20]. There is some argument about the use of extra port that it contradicts the concept of SIPES.

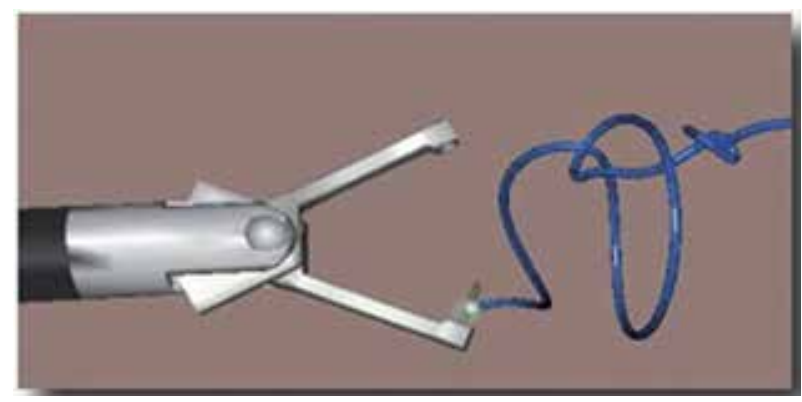

Figure 2.

Endo Stitch device. 
There are some options to replace this port by using tug-exposure or suture suspension techniques [21, 22].

\subsection{SIPES inguinal herniorrhaphy}

Our technique for inguinal herniorrhaphy is percutaneous internal ring suturing (PIRS). The child is placed on supine position, and the surgeon stands on contralateral site of hernia. Laparoscopic camera is inserted through supra umbilical incision. Both sides of deep inguinal ring are explored. Stab wound is placed on the skin crease above deep inguinal ring. Epidural needle inserted in pre-peritoneal area with looped $4 / 0$ nonabsorbable suture. In female the suture surrounds the sac entirely. In boy the needle enters the peritoneum adjacent to vas or vessels for exchange of the suture.

\subsection{SIPES cystectomy/oopherectomy}

We are using bronchoscope with foreign body retrieval forceps to retrieve the cyst and deliver it from the wound in neonate. In older children we use SIPES port and operate as in conventional laparoscopy.

\subsection{SIPES fundoplication}

We insert liver retractor directly through stab wound. The dissection is carried out as in conventional laparoscopy, and traction suture around the esophagus is taken out from the abdominal wall. We performed two cases with large hiatus hernia. Intracorporeal suturing is done by Endo Stitch device (Figure 2).

\subsection{SIPES-assisted Mitrofanoff appendicovesicostomy}

We ligate the base of appendix by extracorporeal sutures instead of endo-loop. The urinary bladder filled to come near the umbilical wound. The operation finished through the umbilical incision.

\subsection{Obesity and SIPES}

In the beginning adult surgeons were doing SILS cholecystectomy on patients with BMI less than 34. Later with improvement of instrumentations and development of disposable and reusable SILS trocars, SILS is now is recommended technique for obese, and it is used for sleeve gastrectomy.

\section{Summary}

- Many procedures have been done in our center

- We found out that appendectomy, cholecystectomy, splenectomy, cystectomy, oopherectomy, orchidectomy, Mitrofanoff, nephrectomy, herniorrhaphy, adhesiolysis, malrotation, and Morgagni hernia are easier to be performed

- Sleeve gastrectomy and fundoplication are less frequently done in our center; the reason in fundoplication is difficulty in suturing.

- This difficulty in older age group could be overcome with Endo Stitch instrument or the use of extracorporeal sliding notes. 


\section{Outcomes of SIPES at King Fahad Armed Forces Hospital (KFAFH)}

The outcome of our retrospective study of all SIPES cases which was done from 2010 to 2016 is shown in (Table 1 and Figure 3).

\subsection{SIPES splenectomy}

Few centers worldwide are performing SIPES pediatric splenectomy (Table 2). On extensive review there were only 166 SILS splenectomy cases, out of them only 61 SIPES pediatric splenectomy published in English, German, Greek, French, Italian, or Spanish literatures in all age groups (0.6-90 years) [23-28]. No publications regarding the safety of SIPES splenectomy were performed by trainees. Laparoscopic splenectomy is a demanding technique which needs high level of skills and mainly performed by the most experienced trained surgeon. Our SIPES splenectomy series is the largest one. 49 SIPES splenectomies were performed by 15 trainees. 25 (51\%) were male and 24 (49\%) were female. Mean age was 6.9 years (2.5-14.8 years). Six cholecystectomies were done simultaneously. 45 patients were having sickle cell disease, two with thalassemia, one spherocytosis, and one Fanconi's anemia. MOT for splenectomy was $182 \mathrm{~min}$ (130-190) and $251 \mathrm{~min}$ for splenectomy with cholecystectomy (230-270) min depending on severity of adhesions and size of the spleen

\begin{tabular}{|c|c|c|c|c|}
\hline Procedures & Age & $\begin{array}{c}\text { OT } \\
(\min )\end{array}$ & \# & Conversion \\
\hline $\begin{array}{l}\text { Cholecystectomy (one with inguinal hernia } \\
\text { repair+ } 1 \text { with appendectomy) }\end{array}$ & $7-16$ & $85 \pm 40$ & 52 & $\begin{array}{c}2 \text { (extra } \\
\text { port) }\end{array}$ \\
\hline Splenectomy (6 with cholecystectomy) & $2.5-14.8$ & $130-270$ & 49 & 2 \\
\hline $\begin{array}{l}\text { Appendectomy (carcinoid, adhesion, } 6 \\
\text { perforated, } 4 \text { appendix mass) }\end{array}$ & $3-12$ & $70-160$ & 137 & 0 \\
\hline $\begin{array}{l}\text { SIPES hernia repair percutaneous internal ring } \\
\text { suturing (PIRS) }\end{array}$ & $\begin{array}{l}2 \text { months }-12 \\
\text { years }\end{array}$ & $20-45$ & 46 & $\begin{array}{l}22 \text { (extra } \\
\text { port) }\end{array}$ \\
\hline $\begin{array}{l}\text { Fundoplication (liver retract, Endo Stitch, } 2 \\
\text { hiatal hernia) }\end{array}$ & $\begin{array}{l}18 \text { months-7 } \\
\text { years }\end{array}$ & $90-180$ & 8 & 0 \\
\hline $\begin{array}{l}\text { Ovarian cystectomy (oophorectomy) ( } 2 \\
\text { teratomas) }\end{array}$ & $\begin{array}{l}4 \text { days-13 } \\
\text { years }\end{array}$ & $30-90$ & 10 & 0 \\
\hline Intussusception & 10 months & 90 & I & 0 \\
\hline Malrotation (Ladd's procedure) & $\begin{array}{c}16 \text { and } 17 \\
\text { years }\end{array}$ & $\begin{array}{l}90 \text { and } \\
120\end{array}$ & 2 & 0 \\
\hline $\begin{array}{l}\text { Assisted Mitrofanoff one with nephrectomy } \\
\text { and orchidectomy }\end{array}$ & $1.5-13$ years & $90-180$ & 10 & 0 \\
\hline SIPES nephrectomy & 18 months & 100 & 1 & 0 \\
\hline SIPES abdominal orchidectomy & 18 months & 20 & 1 & 0 \\
\hline Morgagni hernia & 3 and 5 years & $\begin{array}{l}90 \text { and } \\
120\end{array}$ & 2 & 0 \\
\hline Adhesiolysis & $\begin{array}{c}3 \text { and } 6 \\
\text { years }\end{array}$ & $\begin{array}{c}80 \text { and } \\
100\end{array}$ & 2 & 0 \\
\hline Sleeve gastrectomy & $13-18$ years & $40-120$ & 3 & 0 \\
\hline Total & & & & 324 \\
\hline
\end{tabular}

Table 1.

SIPES outcome at KFAFH. 

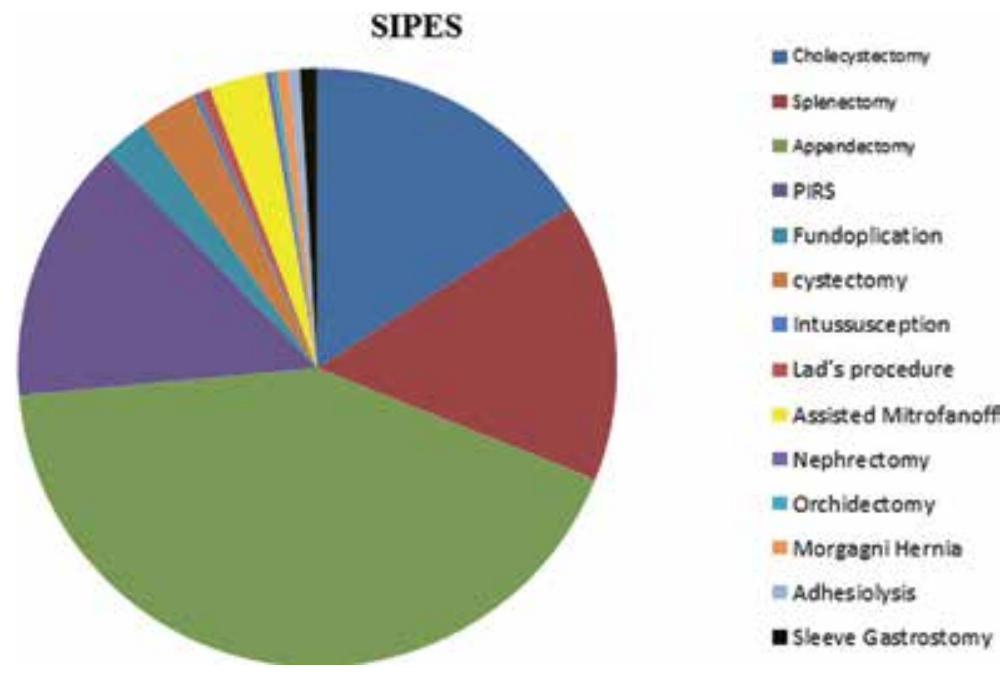

Figure 3.

KFAFH SIPES outcome.

\begin{tabular}{|c|c|c|c|c|c|}
\hline Conversion & Time/min & Detail procedures & $\begin{array}{l}\text { Age } \\
\text { years }\end{array}$ & Number & Authors \\
\hline 0 & 90-176 & $\begin{array}{l}4 \text { splenectomy } \\
2 \text { combined splenectomy/ } \\
\text { cholecystectomy }\end{array}$ & $2-17$ & $\begin{array}{l}20 \text { SIPES } \\
\text { procedures } \\
8 \text { months }\end{array}$ & Dutta [18] \\
\hline 2 (excluded) & 116 & SIPES & $\begin{array}{l}7.1 \\
(2.7-9.7)\end{array}$ & $\begin{array}{l}37 \text { patients } \\
\text { ( } 20 \text { with } \\
\text { extra port) }\end{array}$ & $\begin{array}{l}\text { Seims } \\
\text { et al. [20] }\end{array}$ \\
\hline \multirow[t]{2}{*}{0} & $\begin{array}{l}125 \text { min } \\
\text { (range, } \\
45-420 \\
\text { min) }\end{array}$ & $\begin{array}{l}\text { SILS and SIPES } \\
\text { splenectomy }\end{array}$ & $0.6-90$ & 81 & $\begin{array}{l}\text { Gkegkeset } \\
\text { al [23] }\end{array}$ \\
\hline & & SIPES splenectomy & 6 months & 1 & $\begin{array}{l}\text { Joshi et al. } \\
{[24]}\end{array}$ \\
\hline 2 & 130-190 & SIPES splenectomy & $2.5-14.8$ & 49 & $\begin{array}{l}\text { Raboei } \\
{[25]}\end{array}$ \\
\hline
\end{tabular}

Table 2.

Comparison of SIPES splenectomy in pediatric age group.

P value $<0.001$ (Figures 4 and 5). Two were converted to open due to bleeding and were in the beginning of the series. There is neither wound infection nor incisional hernia update. SIPES splenectomy is now the standard of care in our unit, and we highly recommend it as excellent approach for splenectomy.

\section{Summary}

- This is the first series of SIPES splenectomy for hematological diseases done by trainees.

\begin{tabular}{lcccc}
\hline Procedures & Age & OT (min) & $\#$ & Conversion \\
\hline Splenectomy (six with cholecystectomy) & $2.5-14.8$ & $130-270$ & 49 & 2 \\
\hline
\end{tabular}




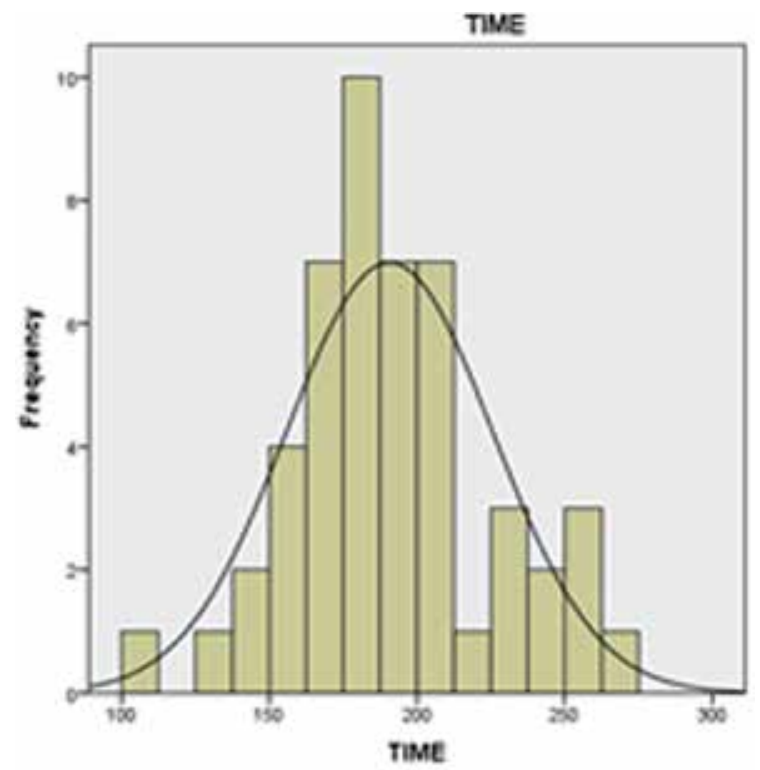

Figure 4.

MOT SIPES splenectomy.

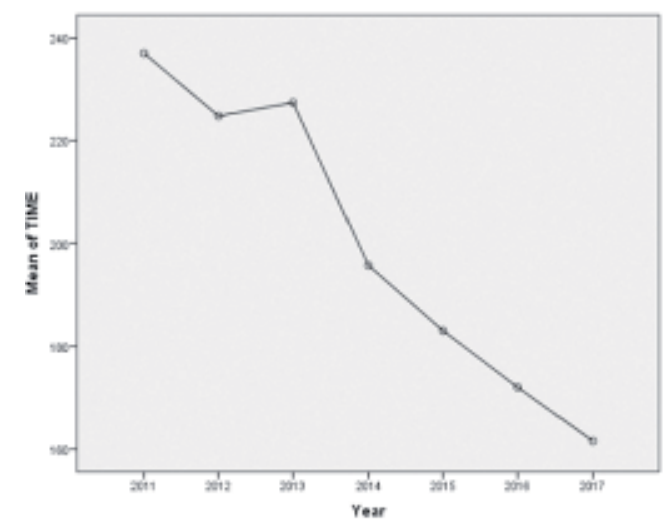

Figure 5.

SIPES MOT over years.

\subsection{SIPES appendectomy}

Most centers advocate laparoscopy for treating acute appendicitis in order to minimize the size and the number of skin incisions even with complicated ones [29]. SIPES pediatric appendectomy has gained significant popularity, as its main advantage is preferable cosmetic result. Previous studies have typically compared SIPES and CLA appendectomy in children and showed heterogeneous results. There was no difference in duration of hospital stay or postoperative complications. Longer MOT with SIPES appendectomy was the main concern [11, 30-41]. One study has shown that SIPES appendectomy in children is safe and feasible when performed by resident doctors and compare it when performed by fellows [42]. Chandler NM et al. studied SIPES versus conventional laparoscopic appendectomy CLA in children. 110 patients underwent appendectomy; 50 SIPES Group 1 and 14 were excluded (perforated) [30, 33]. They concluded that MOT and pain medication are the only significant values between SIPES and conventional. There was no increase in wound infection $[43,44]$. 


\begin{tabular}{lccc}
\hline Operation & Performing surgeon & MOT & NO. \\
\hline SIPES appendectomy & Specialist & 70.723 & 47 \\
\cline { 2 - 4 } & Trainee & 95.289 & 90 \\
\cline { 2 - 4 } & Total & 86.861 & 137 \\
\hline CLA & Specialist & 64.681 & 141 \\
\cline { 2 - 4 } & Trainee & 88.457 & 46 \\
\cline { 2 - 4 } & Total & 70.529 & 187 \\
\hline
\end{tabular}

Table 3.

Performing surgeon and MOT.

We started SIPES appendectomy in 2011, and it became our standard approach for acute appendicitis. It is carried out routinely by trainees and specialist. We compared SIPES appendectomy with CLA between our center and another training center. We conducted retrospective study at two training military institutes, KFAFH and Prince Sultan Medical City PSMMC. A total of 322 patients were operated. 187 patients (58\%) underwent CLA, and 137 patients (42\%) underwent SIPES appendectomy. 120 patients (64\%) of CLA and 87 (63\%) patients of SIPES appendectomy were males. 67 patients (36\%) of CLA and $42(37 \%)$ patients of SIPES appendectomy were females. The mean age of the patients in CLA and SIPES appendectomy was 10 and 9.6 years, respectively. Diagnosis at time of operation was simple appendicitis in 166 patients who underwent CLA and 126 patients who underwent SIPES appendectomy, and complicated appendicitis was in 21 and 11 patients, respectively. 143 CLA (76\%) were performed by specialist, and 44 cases $(24 \%)$ were performed by trainees. 47 SIPES appendectomy (34\%) were performed by specialist, and 90 cases $(66 \%)$ were performed by trainees (Table 3 ) (P value $<0.001)$. The MOT was $70 \mathrm{~min}$ for CLA and $86 \mathrm{~min}$ for SIPES appendectomy ( $\mathrm{P}$ value $<0.001$ ). The mean length of hospital stay is 2.6 days for CLA and 2.8 days for SIPES appendectomy. Bilateral percutaneous internal ring suturing (PIRS) and right PIRS were performed on 2 patients who underwent SIPES appendectomy. Three cases developed intraabdominal collection (2.2\%), one case had wound infection $(0.7 \%)$, and two cases had adhesion (1.5\%), treated conservatively in SIPES appendectomy. There were five cases of intra-abdominal collection (2.7\%), two cases of wound infection (1.1\%), and one case of adhesion ( $0.5 \%)$ in CLA. Negative appendectomy rate has been $10 \%$.

Summary

- SIPES appendectomy is the commonest procedure done in our center by our trainees.

\begin{tabular}{llllc}
\hline Procedures & Age & OT (min) & $\#$ & Conversion \\
\hline $\begin{array}{l}\text { Appendectomy (carcinoid, adhesion, six } \\
\text { perforated, four appendix mass) }\end{array}$ & $3-12$ & $70-160$ & 137 & 0 \\
\hline
\end{tabular}

\subsection{SIPES cholecystectomy}

Minimally invasive techniques have revolutionized surgical treatment. Few centers worldwide have advanced SIPES for pediatric age group. Many papers that were published in English literatures proved the safety and efficacy of SIPES cholecystectomy [44-48]. Up to our knowledge, there is no study regarding MOT of SIPES 


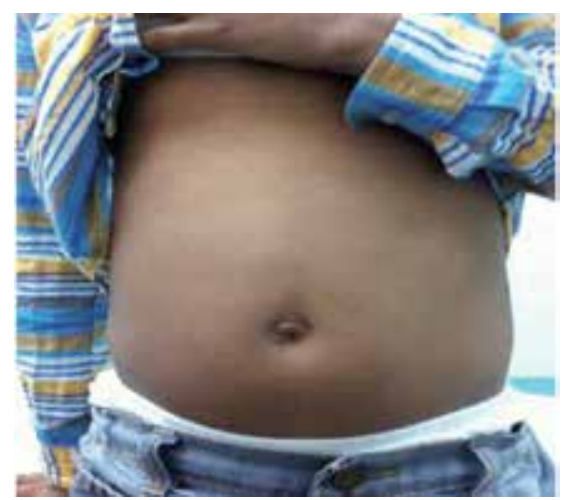

Figure 6.

SIPES cholecystectomy 6 months post-op.

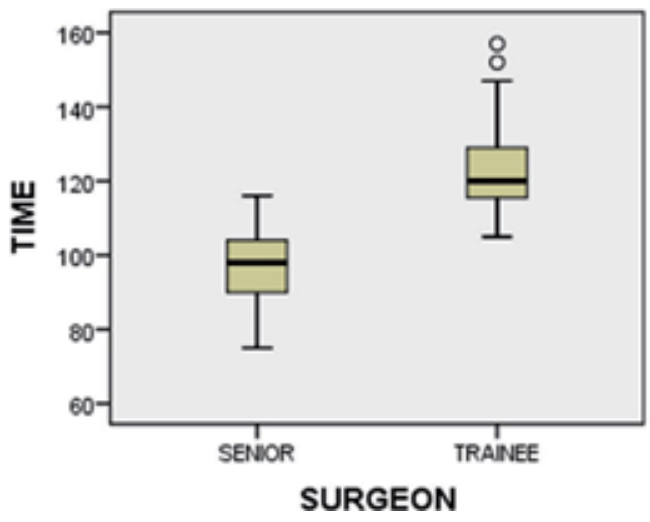

Figure 7.

SIPES cholecystectomy MOT.

cholecystectomy performed by trainees in patients with hematological disease. We conducted a retrospective study to determine the feasibility, safety, and expediency of SIPES cholecystectomy performed by trainees. 45 patients underwent SILS cholecystectomy. Age of the patients ranged from 7 to 16 years. Hematological disease were in $>80 \%$ of cases. One extra port was needed in 2 patients in the beginning of the series. Eight procedures were done simultaneously, six splenectomy, one appendectomy, and one herniotomy, and were excluded from MOT. No conversion was needed. No wound infection. MOT is $85 \pm 40 \mathrm{~min}$. (Figures 6 and 7). It is comparable with conventional technique.

\section{Summary}

- SIPES cholecystectomy is the second common operation in our center.

- We made a new technique for gallbladder traction by using a curved needle which visualize Calot's triangle clearly.

\begin{tabular}{lllll}
\hline Procedures & Age & OT (min) & $\#$ & Conversion \\
\hline $\begin{array}{l}\text { Cholecystectomy (one with inguinal hernia } \\
\text { repair + 1 with appendectomy) }\end{array}$ & $7-16$ & $85 \pm 40$ & 52 & $\begin{array}{l}2 \text { (extra } \\
\text { port) }\end{array}$ \\
\hline
\end{tabular}




\subsection{SIPES inguinal herniorrhaphy}

The approach to inguinal hernia in the pediatric population has historically been via an open technique. During recent years, laparoscopic surgery has emerged as an alternative in the treatment of pediatric inguinal herniorrhaphy. Different laparoscopic technique has been used, and SIPES is a recommended approach for management of inguinal herniorrhaphy [28]. SIPES inguinal herniorrhaphy gives opportunity to explore the contralateral side. We retrospectively review all SIPES inguinal hernia cases. MOT, intra- and postoperative complications, cosmetic results, and contralateral patencies of processus vaginalis (CPPV) were recorded. Patients were followed up for 4 months postoperatively. A total of 46 patients were operated, 39 (84.8\%) were male, and 7 (15.2\%) were female. Right side inguinal hernias were 18 (39.1\%), left were 12 (26.1\%), and bilateral sides were 16 (34.8\%). $56.5 \%$ were less than 1 year, $19.6 \%$ were between 1 and 5 years, and $23.9 \%$ were 6-13 years old. Mean age is 2.7 years. For unilateral procedures MOT was $27 \mathrm{~min}$. For bilateral procedures MOT was 40 minutes. Seven (23\%) (CPPV) was found in laparoscopic surgery. Stitch granuloma developed in one patient.

Summary

- PIRS is comparable with $\mathrm{OH}$.

- It needs good ancillary services for babies below 6 months of age.

\begin{tabular}{lllll}
\hline Procedures & Age & OT $(\mathbf{m i n})$ & $\#$ & Conversion \\
\hline $\begin{array}{l}\text { SIPES hernia repair } \\
\text { percutaneous internal ring } \\
\text { suturing (PIRS) }\end{array}$ & 2 & $20-45$ & 46 & 22 (extra port) \\
\hline
\end{tabular}

\section{Conclusion}

SIPES has excellent cosmesis and almost invisible scar.

We can do more than one procedure at the same time with SIPES technique.

SIPES splenectomy is safe and feasible when performed by surgical trainees without adding any morbidity to the patients even those with hematological diseases.

SIPES appendectomy is feasible and safe in complicated appendicitis.

SIPES cholecystectomy MOT is comparable with conventional technique.

SIPES PIRS for inguinal herniorrhaphy is safe and efficient with good learning curve and best cosmetic result.

SIPES technique can be imparted satisfactorily to trainees. However, its successful incorporation into surgical training programs will depend on the development of innovative simulation strategies.

Our aim is to standardize this approach and to reassure our colleagues regarding the safety, feasibility, and technical challenges of SIPES.

\section{Acknowledgements}

We would like to acknowledge Dr Mohamed Al Onazi and his team from PSMC in Riyadh Saudi Arabia for their contribution. 


\section{Conflict of interest}

None.

\section{Author details}

Enaam Raboei ${ }^{1 *}$, Ameen Alsaggaf ${ }^{2}$, Yazeed Owiwi ${ }^{2}$, Syed Salahuddin ${ }^{3}$, Alaa Ghallab ${ }^{2}$ and Mazen Zidan ${ }^{2}$

1 Pediatric Surgery Department, King Fahd Armed Forces Hospital, Jeddah, Saudi Arabia

2 King Fahd Armed Forces Hospital, Jeddah, Saudi Arabia

3 Leeds, UK

*Address all correspondence to: enaamraboei@kfafh.med.sa

\section{IntechOpen}

(C) 2019 The Author(s). Licensee IntechOpen. This chapter is distributed under the terms of the Creative Commons Attribution License (http://creativecommons.org/licenses/ by/3.0), which permits unrestricted use, distribution, and reproduction in any medium, provided the original work is properly cited. (cc) BY 


\section{References}

[1] Vecchio R, MacFayden BV, Palazzo F. History of laparoscopic surgery. Panminerva Medica. 2000;42(1):87-90

[2] Kalloo AN, Singh VK, Jagannath SB, Niiyama H, Hill SL, Vaughn CA, et al. Flexible transgastric peritoneoscopy: A novel approach to diagnostic and therapeutic interventions in the peritoneal cavity. Gastrointestinal Endoscopy. 2004;60(1):114-117

[3] Li P, Chen Z-H, Li Q-G, Qiao T, Tian Y-Y, Wang D-R. Safety and efficacy of single-incision laparoscopic surgery for appendectomies: A meta-analysis. World Journal of Gastroenterology. 2013;19(25):4072-4082

[4] Pelosi MA, Pelosi MA 3rd. Laparoscopic appendectomy using a single umbilical puncture (minilaparoscopy). The Journal of Reproductive Medicine. 1992;37(7):588-594

[5] Saidy MN, Tessier M, Tessier D. Single-incision laparoscopic surgeryHype or reality: A historical control study. The Permanente Journal. 2012;16(1):47-50

[6] Barbaros U, Dinççağ A. Single incision laparoscopic splenectomy: The first two cases. Journal of Gastrointestinal Surgery. 2009;13(8):1520-1523

[7] Dutta S. Early experience with single incision laparoscopic surgery: Eliminating the scar from abdominal operations. Journal of Pediatric Surgery. 2009;44(9):1741-1745

[8] Hansen EN, Muensterer OJ, Georgeson KE, Harmon CM. Singleincision pediatric endosurgery: Lessons learned from our first 224 laparoendoscopic single-site procedures in children. Pediatric Surgery International. 2011;27(6):643-648
[9] De Armas IA, Garcia I, Pimpalwar A. Laparoscopic single port surgery in children using Triport: Our early experience. Pediatric Surgery International. 2011;27(9):985-989

[10] Kocherov S, Lev G, Shenfeld OZ, Chertin B. Laparoscopic single site surgery: Initial experience and description of techniques in the pediatric population. The Journal of Urology. 2011;186(4 Suppl):1653-1657

[11] Oltmann SC, Garcia NM, Ventura B, Mitchell I, Fischer AC. Single-incision laparoscopic surgery: Feasibility for pediatric appendectomies. Journal of Pediatric Surgery. 2010;45(6):1208-1212

[12] Gasior AC, Knott EM, Holcomb GW, Ostlie DJ, St Peter SD. Patient and parental scar assessment after single incision versus standard 3-port laparoscopic appendectomy: Longterm follow-up from a prospective randomized trial. Journal of Pediatric Surgery. 2014;49:120-122. discussion 122

[13] Jayashingh SC. Comparison of advantages and disadvantages between SILS and NOTES. World Journal of Laparoscopic Surgery. 2011;4(1):67-72

[14] Fransen SA, Broeders E, Stassen L, Bouvy N. The voice of Holland: Dutch public and patient's opinion favours single-port laparoscopy. Journal of Minimal Access Surgery. 2014;10:119-125

[15] Chow A, Purkayastha S, Dosanjh D, Sarvanandan R, Ahmed I, Paraskeva P. Patient reported outcomes and their importance in the development of novel surgical techniques. Surgical Innovation. 2012;19(3):327-334. DOI: 10.1177/1553350611426011. Epub 2011 Dec 7

[16] Rivas H, Varela E, Scott D. Singleincision laparoscopic cholecystectomy: 
Initial evaluation of a large series of patients. Surgical Endoscopy.

2010;24(6):1403-1412

[17] Padilla BE, Dominguez G, Millan C, Martinez-Ferro $M$. The use of magnets with single-site umbilical laparoscopic surgery. Seminars in Pediatric Surgery. 2011;20:224-231

[18] Dutta S. Early experience with single incision laparoscopic surgery: Eliminating the scar from abdominal operations. Journal of Pediatric Surgery. Sep 2009;44(9):1741-1745

[19] Blanco FC, Kane TD. Single-port laparoscopic surgery in children: Concept and controversies of the new technique. Minimally Invasive Surgery. 2012;2012:232347. Published Online 2012 Jun 12

[20] Seims AD, Russell RT, Beierle EA, Chen MK, Scott AA, Martin CA, et al. Single-incision pediatric endosurgery (SIPES) splenectomy: What dictates the need for additional ports? Surgical Endoscopy. 2015;29(1):30-33

[21] Misawa T, Sakamoto T, Ito R, Shiba $\mathrm{H}$, Gocho T, Wakiyama S, et al. Singleincision laparoscopic splenectomy using the "tug-exposure technique" in adults: Results of ten initial cases. Surgical Endoscopy. 2011;25(10):3222-3227

[22] Li S, Li M, Xu W, Sun C, Liu L. Single-incision laparoscopic splenectomy using the suture suspension technique for splenomegaly in children with hereditary spherocytosis. Journal of Laparoendoscopic \& Advanced Surgical Techniques. Part A. 2015;25(9):770-774

[23] Gkegkes D, Mourtarakos S, Iavazzo C. Single-incision laparoscopic splenectomy. JSLS. 2014;18(3):e2014.00350

[24] Joshi M, Kurhade S, Peethambaram M, Kalghatgi S, Narsimhan M,
Ardhanari R. Single-incision laparoscopic splenectomy. Journal of Minimal Access Surgery. 2011;7(1):65-67

[25] Raboei E, Owiwi Y, Ghallab A, Zeinelabdeen A, Alsaggaf A, et al. Is It Safe for Trainees to Perform Single-Incision Pediatric Endosurgery Splenectomy?. Journal of Laparoendoscopic \& Advanced Surgical Techniques. Mar 2019;29(3):420-423

[26] Hansen EN, Muensterer OJ. Single incision laparoscopic splenectomy in a 5-year-old with hereditary spherocytosis. JSLS. 2010;14(2):286-288

[27] Garrett D, Emami C, Anselmo DM, Torres MB, Nguyen N. Single-incision laparoscopic approach to management of splenic pathology in children: An early experience. Journal of Laparoendoscopic \& Advanced Surgical Techniques. Part A. 2011;21:965-967

[28] Saldana LJ, Targarona EM. Singleincision pediatric endosurgery: A systematic review. Journal of Laparoendoscopic \& Advanced Surgical Techniques. Part A. 2013;23:467-480

[29] Muensterer O, Puga Nougues C, Adibe O, Amin S, Georgeson K, Harmon C. Appendectomy using single-incision pediatric endosurgery for acute and perforated appendicitis. Surgical Endoscopy. 2010;24(12):3201-3204

[30] Chandler NM, Danielson PD. Single-incision laparoscopic appendectomy vs multiport laparoscopic appendectomy in children: A retrospective comparison. Journal of Pediatric Surgery. 2010;45(11):2186-2190

[31] Chow A1, Aziz O, Purkayastha S, Darzi A, Paraskeva P. Single incision laparoscopic surgery for acute appendicitis: Feasibility in pediatric patients. Diagnostic and Therapeutic Endoscopy. 2010;2010:294958 
[32] Lacher M, Muensterer OJ, Yannam GR, Aprahamian CJ, Perger L, Megison $\mathrm{M}$, et al. Feasibility of single-incision pediatric endosurgery for treatment of appendicitis in 415 children. Journal of Laparoendoscopic \& Advanced Surgical Techniques. Part A. 2012;22(6):604-608

[33] Chandler NM, Danielson PD. SILS versus conventional laparoscopic appendectomy CLA in children. Journal of Pediatric Surgery. 2010;45(11):2186-2190

[34] Perez EA, Piper H, Burkhalter LS, Fischer AC. Single-incision laparoscopic surgery in children: A randomized control trial of acute appendicitis. Surgical Endoscopy. 2013;27:1367-1371

[35] Salö M, Järbur E, Hambraeus M, Ohlsson B, Stenström P, Arnbjörnsson E. Two-trocar appendectomy in children-Description of technique and comparison with conventional laparoscopic appendectomy. BMC Surgery. 2016;16:521-525

[36] Wu K, Yang L, Wu A, et al. Single-site laparoscopic appendectomy in children using conventional instruments: A prospective, randomized, control trial. Pediatric Surgery International. 2015;31:167-171

[37] Duza G, Palermo M, Khiangte E, Azfar M, Rizvi SAA, Trelles N, et al. Single port laparoscopic appendectomy vs conventional laparoscopic appendectomy: Outcomes after multicenter randomized control trial. Journal of American College of Surgeons. 2015;221(4 Suppl 2):e17

[38] St. Peter S, Adibe O, Juang D, Sharp S, Garey C, Laituri C, et al. Single incision versus standard 3-port laparoscopic appendectomy. Annals of Surgery. 2011;254(4):586-590

[39] Feng J, Cui N, Wang Z, Duan J. Bayesian network meta-analysis of the effects of single-incision laparoscopic surgery, conventional laparoscopic appendectomy and open appendectomy for the treatment of acute appendicitis. Experimental and Therapeutic Medicine. Dec 2017;14(6):5908-5916

[40] Park J, Kwak H, Kim SG,

Lee S. Single-port laparoscopic appendectomy: Comparison with conventional laparoscopic appendectomy. Journal of Laparoendoscopic \& Advanced Surgical Techniques. Part A. 2012;22:142-145

[41] Yannam G, Griffin R, Anderson S, Beierle E, Chen M, Harmon C. Single incision pediatric endosurgery (SIPES) appendectomy-Is obesity a contraindication? Journal of Pediatric Surgery. 2013;48(6):1399-1404

[42] Wakasugi M, Tsujimura N, Nakahara Y, Matsumoto T, Takemoto H, Takachi K, et al. Single-incision laparoscopically assisted appendectomy performed by residents is safe and feasible: A single institution, retrospective case series. Annals of Medicine and Surgery. 2017;15:43-46

[43] Sesia S, Haecker F, Kubiak R, Mayr J. Laparoscopy-assisted singleport appendectomy in children: Is the postoperative infectious complication rate different? Journal of Laparoendoscopic \& Advanced Surgical Techniques. 2010;20(10):867-871

[44] Stringe G, Zitsman JL, Shehadi I, Kithir S. Laparoscopic Appendectomy in Children. JSLS. 1997;1(1):37-39

[45] Navarra G, Pozza E, Occhionorelli S, Carcoforo P, Donini I. One-wound laparoscopic cholecystectomy. The British Journal of Surgery. 1997;84(5):695

[46] Podolsky ER, Rottman SJ, Poblete H, King SA, Curcillo PG. Single port access (SPA) cholecystectomy: A completely transumbilical approach. 
Journal of Laparoendoscopic \&

Advanced Surgical Techniques. Part

A. 2009;19(4):495-499. DOI: 10.1089/

lap.2008.0424

[47] Podolsky ER, Curcillo PG 2nd.

Reduced-port surgery: Preservation of the critical view in single-port-access cholecystectomy. Surgical Endoscopy. 2010;24(12):3038-3043

[48] Podolsky ER, Rottman SJ, Curcillo PG 2nd. Single port access (SPA)

cholecystectomy: Two year follow-up. JSLS. 2009;13(4):528-535 

